\title{
Roles of Methyl-CpG Binding Protein 2 in Immune Function
}

\author{
James Christopher Cronk \\ Lafayette, Colorado
}
B.A. Molecular, Cellular, Developmental Biology and Anthropology (Double Major) University of Colorado, Boulder, 2008

\begin{abstract}
A Dissertation presented to the Graduate Faculty of the University of Virginia in Candidacy for the Degree of

Doctor of Philosophy

Department of Neuroscience
\end{abstract}

University of Virginia

May 2016 


\section{Abstract}

Methyl-CpG binding protein 2 (MeCP2) was one of the first methyl-CpG binding proteins identified. Since its discovery, $\mathrm{MeCP} 2$ has been shown to be an essential regulator of gene transcription via its role in orchestrating transcriptional complexes associated with methylated CpG islands in the genome. In 1999, mutations in MeCP2 were identified as the primary cause of Rett syndrome. Several years later, another MeCP2-related neurodevelopmental disorder was identified, called MeCP2 duplication syndrome. Due to the clear association of both disorders with neurologic pathology, and the high levels of MeCP2 expressed by neurons, it was largely assumed that the complete etiology of disease in both disorders was explained by defects in neuronal function. However, it has since become clear that many cells throughout all tissues express and require $\mathrm{MeCP} 2$ for their normal function. Thus, understanding both the basic biology and disease relevance of $\mathrm{MeCP} 2$ in non-neuronal cell types has become an important question. This is particularly true for the immune system, where nearly every cell expresses MeCP2.

Presented here is evidence for the importance of MeCP2 in function of the immune system; 1) $\mathrm{MeCP} 2$ regulates microglia and macrophage gene expression in response to multiple stimuli, and 2) overexpression of $\mathrm{MeCP} 2$ leads to a dysfunctional immune response and mortality upon influenza A infection. Together, these results demonstrate that $\mathrm{MeCP} 2$ is an important regulator of immunity in multiple contexts, and thus immune roles for $\mathrm{MeCP} 2$ should be considered as a fundamental aspect of $\mathrm{MeCP} 2$ function. 


\section{Table of contents}

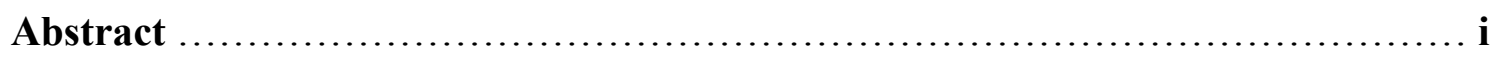

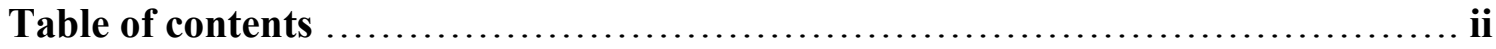

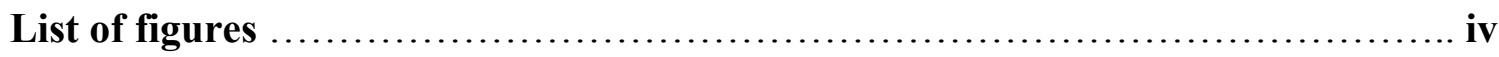

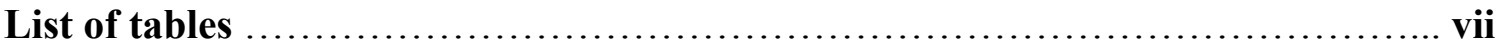

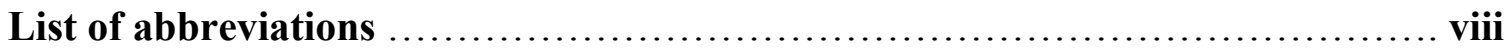

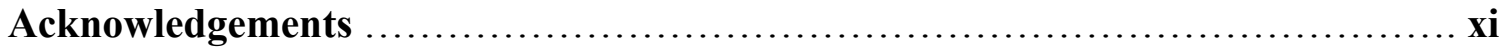

Chapter 1: Introduction ................................................. 1

The discovery and function of $\mathrm{MeCP} 2$.......................... I

Clinical features and natural history of Rett syndrome ..............4

The genetic basis of Rett syndrome .............................. 7

MeCP2 duplication syndrome ................................ 10

Mouse models of Rett syndrome and the role of MeCP2 in neurons ... 14

The role of $\mathrm{MeCP} 2$ in astrocytes and oligodendrocytes ............... 21

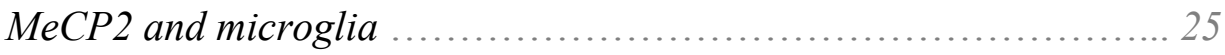

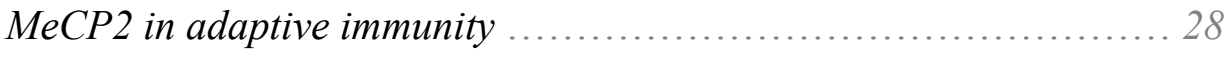

Thesis rationale ................................................... 31

Chapter 2: Materials and methods ........................................... 34 
Chapter 3: MeCP2 regulates transcriptional response

to stimuli in macrophages

Summary of results .............................................. 46

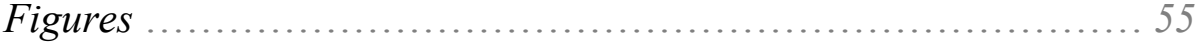

Chapter 4: Lethal outcome and dysfunctional immune response to

influenza $A$ in an animal model of MeCP2 overexpression ........... 61

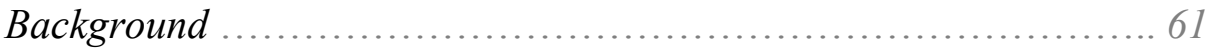

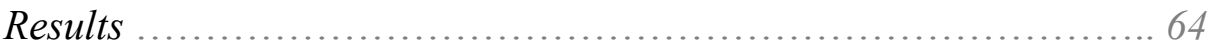

Discussion ................................................. 71

Figures and tables .......................................... 75

Chapter 5: Summary discussion and future directions ......................89

$A$ reassessment of $\mathrm{MeCP} 2$ in health and disease .................8 89

Regulation of responses to stimuli in immune cells by MeCP2 ....... 92

A complex network of cell-intrinsic and extrinsic functions .......... 96

MeCP2 as a multifunctional orchestrator of immune response ....... 98

MeCP2 and the immune system in human disease ................. 101

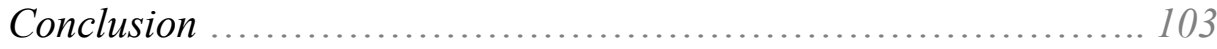

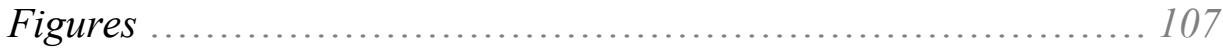

Appendix: Methyl-CpG binding protein 2 regulates microglia and macrophage gene expression in response to inflammatory stimuli ................ 111

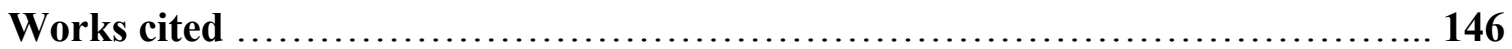




\section{List of figures}

Figure 1. Nestin ${ }^{\text {Cre }}$ mediated deletion of Mecp2 does not fully recapitulate the reduced survival observed in Mecp2-null mice

Figure 2. Flow cytometric staining for Mecp2 in microglia of Mecp2-heterozygous female mice reveals chimeric expression of Mecp2 in microglia

Figure 3. Mcsf treatment does not improve survival in Mecp2-null mice 57

Figure 4. Wild type monocyte transfer does not improve survival in Mecp2-null mice $\mathbf{5 8}$

Figure 5. LPS induces Mecp2 expression in BMDM, which is attenuated by priming with IFN $\gamma$ 60

Figure 6. $\mathrm{MeCP} 2^{T g 3}$ mice naturally start to die after 200 days of age, and have no obvious baseline peripheral immune defects.

Figure 7. $\mathrm{MeCP} 2^{T g 3}$ mice are highly susceptible to influenza A infection, with late adaptive immune failure and uncontrolled virus titers

Figure 8. $M e C P 2^{T g 3} T$ cells display strong activation early during infection, but by day 8 both total and influenza-specific T cells are deficient, in addition to reduced IFN $\gamma$ production

Figure 9. Peripheral and pulmonary neutrophilia associated with mortality occurs on day 5 p.i. in $\mathrm{MeCP} 2^{T g 3}$ mice 81

Figure 10. MeCP2 $2^{T g 3}$ mice develop pulmonary cytokine storm, and $\mathrm{COX}-2$ inhibition corrects neutrophilia and reduces weight loss but does not improve survival 
Figure 11. Corticosterone is significantly elevated starting day 5 post infection, however does not explain mortality ............................., 85

Figure 12. The MeCP2 $2^{T g 3}$ immune system is insufficient to cause enhanced mortality to influenza A infection, and influenza A infection in $M e C P 2^{T g 3}$ mice leads to vascular pathology ..........................................86

Figure 13. Vascular pathology at day 7 post infection in $M e C P 2^{T g 3}$ mice ..............88

Figure 14. Schematic of potential pathologic synergy between neuronal and non-neuronal cell types during disease progression of Mecp2-deficient mice ...................................... 107

Figure 15. Schematic of pathologic events during influenza A infection in $\mathrm{MeCP} 2^{T g 3}$ mice .................................................. 109 
The following figures appear in the Appendix

Figure A1. Microglia become activated and subsequently depleted with disease progression in Mecp2-null mice

Figure A2. Meningeal macrophages are lost with disease progression in Mecp2-null mice 116

Figure A3. Peripheral monocytes and macrophages express Mecp2, and some are lost in Mecp2-null mice

Figure A4. Postnatal expression of Mecp2 via CX3cr1 ${ }^{\text {CreER }}$ in otherwise Mecp2-deficient mice increases lifespan

Figure A5. Mecp2 regulates glucocorticoid and hypoxia responses in microglia and peritoneal macrophages

Figure A6. Mecp2 restrains inflammatory responses in macrophages

Figure AS1. Representative images of wild type and late-phenotypic Mecp2-null microglia

Figure AS2. BMDM generated per monocyte in wild type and Mecp2-null 128

Figure AS3. NCOR2 and HDAC3 occupancy profiles at the Fkbp5 gene promoter ...

Figure AS4. Intraperitoneal saline injection into Mecp2-null mice does not effect gene expression of TNF-induced transcripts 130

Figure AS5. Mecp2-null mice have progressive neutrophilia and hematopoietic stem cell loss, which can be attenuated by anti-GCSF neutralizing antibodies 


\section{List of tables}

Table 1. Luminex analysis of BAL fluid in wild type and $M e C P 2^{T g 3}$, day 5 p.i. ....... 75 


\section{List of abbreviations}

$\begin{array}{ll}\text { 5hmC } & \text { 5-hydroxymethylcytosine } \\ \text { 5mC } & \text { 5-methylcytosine } \\ \text { APC } & \text { Antigen presenting cell } \\ \text { ASD } & \text { Autism spectrum disorder } \\ \text { BAL } & \text { Bronchoalveolar lavage } \\ \text { BDNF } & \text { Brain-derived neurotrophic factor } \\ \text { BMDC } & \text { Bone marrow-derived dendritic cell } \\ \text { BMDM } & \text { Bone marrow-derived macrophage } \\ \text { CBC } & \text { Complete blood count } \\ \text { ChIP } & \text { Chromatin immunoprecipitation } \\ \text { CNS } & \text { Central nervous system } \\ \text { COX-2 } & \text { Cyclooxygenase-2 } \\ \text { Csf3 } & \text { Granulocyte-colony stimulating factor, mouse gene } \\ \text { Cx3cr1 } & \text { Chemokine (C-X3-C motif) receptor 1 } \\ \text { CXB } & \text { Celecoxib } \\ \text { Cxcl5 } & \text { Chemokine (C-X-C motif) ligand 5 } \\ \text { DSM-5 } & \text { Diagnostic and statistical manual of mental disorders 5 } \\ \text { ELISA } & \text { Enzyme-linked immunosorbent assay } \\ \text { Fkbp5 } & \text { FK506 binding protein 5 } \\ \text { Foxp3 } & \text { Forkhead box P3 } \\ \text { Gcsf } & \text { Granulocyte-colony stimulating factor, mouse protein } \\ \text { GFAP } & \text { Glial fibrillary acidic protein } \\ \text { HDAC } & \text { Histone deacetylase } \\ \text { H\&E } & \text { Hematoxylin and eosin } \\ \text { HSC } & \text { Interferon gammatopoietic stem cell } \\ \text { IFNy } & \text { Immunoglobulin A } \\ \text { IgA } & \text { IgG }\end{array}$




\begin{tabular}{|c|c|}
\hline IgM & Immunoglobulin $\mathrm{M}$ \\
\hline i.p. & Intraperitoneal \\
\hline iPSC & Induced pluripotent stem cell \\
\hline $\operatorname{IRAK1}$ & Interleukin-1 receptor-associated kinase 1 \\
\hline LPS & Lipopolysaccharide \\
\hline MDCK & Madin-Darby canine kidney cells \\
\hline МeCP2 & Methyl-CpG binding protein 2 , human protein \\
\hline $\mathrm{MeCP2}$ & Methyl-CpG binding protein 2, human gene \\
\hline Мecp2 & Methyl-CpG binding protein 2 , mouse protein \\
\hline Mecp2 & Methyl-CpG binding protein 2, mouse gene \\
\hline MFP & Mifepristone \\
\hline Mesf & Macrophage-colony stimulating factor \\
\hline Ng2 & Neuron-glial antigen 2 \\
\hline NPC & Neural progenitor cell \\
\hline OVA & Ovalbumin \\
\hline PBMC & Peripheral blood mononuclear cell \\
\hline PFA & Paraformaldehyde \\
\hline p.i. & Post-infection \\
\hline RBC & Red blood cell \\
\hline ROS & Reactive oxygen species \\
\hline RSS & Rett severity scale \\
\hline SEM & Standard error of the mean \\
\hline SLE & Systemic lupus erythematosus \\
\hline Snat1 & Sodium-coupled neutral amino acid transporter 1 \\
\hline Socs5 & Suppressor of cytokine signaling 5 \\
\hline Stat1 & Signal transducer and activator of transcription 1 \\
\hline Stat3 & Signal transducer and activator of transcription 3 \\
\hline $\operatorname{Tg} f b 1$ & Transforming growth factor beta 1 , mouse gene \\
\hline Th1 & T helper 1 \\
\hline Th17 & T helper 17 \\
\hline
\end{tabular}


Tnf

TNF

Tnf

Treg
Tumor necrosis factor, mouse protein

Tumor necrosis factor, human protein

Tumor necrosis factor, mouse gene

Regulatory $\mathrm{T}$ cells 


\section{Acknowledgements}

The completion of my $\mathrm{PhD}$ would not have been possible without the help of so many individuals. It would be inappropriate to first thank anyone other than my best friend and wife, Meghan Elizabeth Cronk. I couldn't have asked for more patience and understanding; the support you have shown me is something I could never hope to repay you for, although I will try in earnest to do just that. Likewise, I thank my family; my mother Mary, my father Chris, and my brothers Dan and John, for their constant support and love through the good and the bad.

I thank my advisor, Jony Kipnis, for your patience with my idiosyncrasies, your time spent teaching me skills I will take with me for the rest of my career, and your constant pushing and encouraging to go to the next level, doing things I never would have thought myself capable of without your support and belief in me. Thank you to Noel Derecki for teaching me many of the lab skills I learned in graduate school, and for being someone I could depend and rely on, both personally and professionally. I thank both Noel and Jony for their friendship and their solidarity throughout the years.

I thank the members of my committee for their advice and guidance along the way: Kevin Lee, Tom Braciale, Ken Tung, Tajie Harris, and Howard Goodkin.

I would like to acknowledge and thank the members of the Kipnis lab, all of whom I consider to be friends as well as colleagues. To my fellow graduate students, Geoff Norris, Ioana Marin, Jamie Walsh, Ali Radjavi, and Sachin Gadani; to the post docs who were all willing to help and teach me, Antoine Louveau, Tony Filiano, and Jasmin Herz; to Emily Ji, whose contributions were essential to the success of these 
projects; and of course to our lab manager Wendy Baker, and our veterinarian Igor Smirnov.

To our collaborators, and those who helped me with my projects, conducted experiments that appear in this dissertation and published works, and were willing to spend their time discussing my research, I am truly indebted. I am sure I will unintentionally forget some people who lent their help and support to me in this journey, so to them, I say thank you, and I apologize if I have forgotten to put your name here. So to the members of the Litvak lab, Vladimir Litvak, Amy (Xu) Yang, an Aaron Lampano; the members of the Braciale lab - especially Tom Braciale, who spent his valuable time acting as a co-mentor to me in all my infection studies - Taeg Kim, Emily Moser, and Barbara Small; Ashish Sharma; Sandy Feldman; Joanne Lannigan; Ken Tung; Stephen Turner; Tajie Haris; and Alexander Kilbanov, I am truly grateful to you all.

To the MSTP, the NGP, the BIG center, and the Department of Neuroscience especially the people who have helped me navigate this difficult journey: Sharon Heyka, Janine Vallee, Joni Capelle, and Dori Williams - I appreciate you all.

To our friends outside the lab who have been there for both Meghan and I over the years, thank you as well, especially Dave and Mer Peske, and Joe and Lex Walpole, who made us feel welcome and embraced before we even made the move to Charlottesville, and who have continued to be wonderful friends to us ever since.

Finally, I would like to thank the mice, without which it would be impossible to obtain the knowledge presented here. 


\section{Chapter 1}

\section{Introduction}

\section{The discovery and function of $\mathrm{MeCP} 2$}

Methyl-CpG binding protein $2(\mathrm{MeCP} 2)$ was discovered and characterized in the lab of Adrian Bird in 1992 (Lewis et al., 1992; Meehan et al., 1992). It was one of the first proteins discovered that specifically bound methylated $\mathrm{CpG}$ sites in the genome, and thus at the time, the function of these proteins was not well understood. Methyl-CpG binding proteins have since become known as one of the most important epigenetic regulators of gene expression in eukaryotic cells (Jaenisch and Bird, 2003). Typically, such regulation occurs in $\mathrm{CpG}$ islands, which are on average 1000 base pairs long and contain a high density of cytosine and guanine, and thus a high density of CpG sites (Deaton and Bird, 2011). CpG islands are usually located within or proximal to the promoter of mammalian 
genes in the 5' region and, when methylated, allow for methyl-CpG binding proteins to bind the site (Deaton and Bird, 2011). Methyl-CpG binding proteins, including MeCP2, have been shown to mediate transcriptional repression when bound to the methylatedCpG islands proximal to the gene in question (Boyes and Bird, 1991, 1992; Jones et al., 1998; Nan et al., 1998; Yu et al., 2000). MeCP2 binds to a single methylated-CpG, unlike other methyl-CpG binding proteins/complexes that require multiple methylated-CpG sites for their binding to DNA (Meehan et al., 1992). Thus, MeCP2 is poised as a high-affinity and sensitive DNA binding partner, which coats the genome at nearly any available methyl-CpG (Skene et al., 2010).

$\mathrm{MeCP} 2$ exerts transcriptional control via recruitment of binding partners. The first identified binding partner was the co-repressor Sin3a (Jones et al., 1998; Nan et al., 1998), and has since been shown to interact with additional binding partners in conjunction with Sin3a, such as PU.1 (Suzuki et al., 2003). The recruitment of Sin3a by $\mathrm{MeCP} 2$ is also associated with recruitment of histone deacetylases (HDACs), which mediate chromatin remodeling, and thus transcriptional repression of the targeted locus (Jones et al., 1998; Nan et al., 1998). It was also shown that MeCP2 binds the NCoR/SMRT co-repressor complex (Kokura et al., 2001; Lyst et al., 2013), and that this interaction can be controlled in an activity-dependent manner via phosphorylation of the MeCP2 protein (Ebert et al., 2013). MeCP2 additionally interacts with c-Ski (Kokura et al., 2001) and the REST/CoREST repressor complexes (Lunyak et al., 2002). In all, MeCP2 has been well established as a key binding partner for many transcriptional repressors, and this list may continue to grow. 
While transcriptional repression is a clear primary function of $\mathrm{MeCP} 2$, it has also been shown to mediate transcriptional activation (Chahrour et al., 2008). This came as a surprise, because it had previously been thought that MeCP2, like other methyl-CpG binding proteins, functioned only to repress transcription via its binding to methylated $\mathrm{CpG}$ sites in promoter regions. However, the evidence in this work suggests that MeCP2 can also serve as a transcriptional activator when CREB1 functions as a binding partner (Chahrour et al., 2008).

The potential roles of $\mathrm{MeCP} 2$ in epigenetic regulation have been further expanded, as additional nucleotide binding partners (Mellen et al., 2012) and evidence for a more global role in chromatin structure (Skene et al., 2010) have been uncovered. The discovery that MeCP2 binds 5-hydroxymethylcytosine (5hmC) in addition to its classical binding of 5-methylcytosine $(5 \mathrm{mC})$ was not only surprising due to the fundamentally new function for $\mathrm{MeCP} 2$, but also the fact that genes with high $5 \mathrm{hmC}$ content are generally transcriptionally active (Mellen et al., 2012). This gives support to evidence that MeCP2 can mediate transcriptional activation (Chahrour et al., 2008), and suggests that 5hmC binding by $\mathrm{MeCP} 2$ may represent an important component of transcriptional activation by $\mathrm{MeCP} 2$.

Although the evidence is strong that $\mathrm{MeCP} 2$ is a central regulator of transcription, studies examining the overall effect of $\mathrm{MeCP} 2$ on gene transcript levels gave the surprising result that relatively small variations are seen when $\mathrm{MeCP} 2$ is deleted or overexpressed, typically no more than two-fold up or down (Ben-Shachar et al., 2009; Tudor et al., 2002). This result is surprising not only because of MeCP2's involvement in 
a wide-array of transcriptional complexes and ubiquitous binding across the genome (Skene et al., 2010), but also the fact that disruption of MeCP2 leads to severe neurodevelopmental disorders (Amir et al., 1999; Van Esch et al., 2005). This relatively small effect on individual gene transcription is an unsatisfying answer to the question of not only what the role of $\mathrm{MeCP} 2$ is in the larger-scale function of the cell, but also how alterations of its expression can lead to such severe health consequences.

\section{Clinical features and natural history of Rett syndrome}

Rett syndrome was first described by the Austrian neurologist Andreas Rett in 1966 (Rett, 1966), although the syndrome was not coined as such until 1983 when Bengt Hagberg made the connection between Rett's findings and the clinical features of a population of 35 patients (Hagberg et al., 1983). Part of this delay in the recognition of Rett syndrome may have been due to the fact that Rett's initial publication was written in German, while Hagberg's was in English; regardless, Hagberg was kind enough to give Rett credit for first recognizing the syndrome which now bears his name.

Rett syndrome can be best characterized as a progressive neurodevelopmental syndrome, whereby early development is apparently normal, with a sharp decline or delay in developmental progress occurring between 6-18 months of age (Chahrour and Zoghbi, 2007). As the disease progresses, patients continue to experience additional and both accelerating and/or diminishing symptoms with age, until progression may eventually plateau (Chahrour and Zoghbi, 2007). One of the first symptoms is a reduction of head growth, potentially leading to microcephaly by two years of age (Chahrour and 
Zoghbi, 2007; Hagberg, 2002). In general, once symptoms begin, there is significant developmental delay, and while some patients may have begun to learn to walk, or speak some words, regression or failure to progress in these areas ensues (Chahrour and Zoghbi, 2007). In addition to microcephaly, patients experience general growth deficit accompanied by muscle hypotonia, which compounds the growth problems. As the disease progresses, patients will manifest additional neurologic phenotypes, such as hand stereotypies characterized by hand wringing, flapping, patting, clapping etc., which are unique to each patient (Chahrour and Zoghbi, 2007; Hagberg, 2002; Neul et al., 2010). In addition to hand motor deficits, patients also develop both ataxia and gait apraxia, which make typical ambulation difficult, leading to many patients being wheelchair bound (Chahrour and Zoghbi, 2007; Hagberg, 2002). Breathing abnormalities are also prevalent. Girls may experience episodic hyperventilation, breath holding spells, and waking apneas (Chahrour and Zoghbi, 2007; Hagberg, 2002).

One of the most variable, but potentially severe complications is seizure. Compared to other mental retardation syndromes, Rett syndrome has a relatively late onset of epileptic seizures at a median of 4 years in Rett versus 0.8 years of age in other mental retardation (Hagberg, 2002; Steffenburg et al., 2001). Patients may experience partial complex or tonic-clonic seizures, and the severity may range from an easily controlled seizure disorder to intractable epilepsy (Chahrour and Zoghbi, 2007). Although there is significant variability in the severity of seizures, they are particularly penetrant throughout the Rett syndrome population, with one study finding a history of epilepsy in $94 \%$ of Rett subjects (50 out of a cohort of 53 Swedish patients) (Steffenburg 
et al., 2001). Interestingly, seizures typically decrease after the teenage years, and may be a minor problem in adulthood (Chahrour and Zoghbi, 2007).

Girls suffering from Rett syndrome experience impaired social interaction, which is of course exacerbated by deficits in language development. These features were originally characterized as autistic in nature, and thus Rett syndrome was originally included in the autism spectrum disorders (ASD). However, as of the diagnostic and statistical manual of mental disorders 5 (DSM-5), Rett syndrome is no longer included in ASD (American Psychiatric Association. and American Psychiatric Association. DSM-5 Task Force., 2013). This was accompanied by a significant reorganization in the classification of ASD, which included the removal of other previously unique diagnoses, such as Asperger's syndrome, and their lumping into a single ASD diagnosis. Due to the unique nature of Rett syndrome, the decision was made in the DSM-5 not to include Rett syndrome under the single ASD diagnosis.

Additional peripheral features develop as the disease progresses. Scoliosis and osteoporosis are prominent (Chahrour and Zoghbi, 2007; Hagberg, 2002; Motil et al., 2014). In addition, gastrointestinal problems are common, including chewing difficulties, gastroesophageal reflux, constipation, and occasional biliary tract disorders (Hagberg, 2002; Motil et al., 2012). These gastrointestinal complications may significantly contribute to the general growth deficit and nutritional deficiencies observed in Rett syndrome patients (Motil et al., 2012). Autonomic function is also disrupted, including peripheral vascular/vasomotor dysfunction and cardiac abnormalities, with prolonged QT interval and abnormal heart rate representing prominent manifestations of this aspect of 
disease (Chahrour and Zoghbi, 2007; Ellaway et al., 1999; Glaze, 2005; Hagberg, 2002; Neul et al., 2010; Sekul et al., 1994). Patients may also experience bruxism, air swallowing, laughing spells, elevated anxiety, and even Parkinsonian features by the late stages of disease (Chahrour and Zoghbi, 2007; Hagberg, 2002, 2005; Neul et al., 2010; Roze et al., 2007).

Although Rett syndrome results in many severe complications, most patients do survive into their adult years, although these individuals suffer from increasing debilitation and diminished quality of life (Chahrour and Zoghbi, 2007). A British survey found an annual mortality rate of $1.2 \%$, with $26 \%$ sudden, unexpected deaths. $48 \%$ of deaths occurred in debilitated patients and were not sudden or unexpected (Kerr et al., 1997). A potential explanation for the high number of sudden unexpected deaths may be the development of prolonged QT intervals, and thus subsequent catastrophic cardiac arrhythmia may represent a significant cause of mortality (Ellaway et al., 1999; Sekul et al., 1994). Regardless of the specific cause of death in these patients, the majority of girls suffering from Rett syndrome may survive well into their teen and adult years, and must endure the progressive complications of this devastating neurodevelopmental disorder throughout their lives.

\section{The genetic basis of Rett syndrome}

Rett syndrome is primarily caused by mutations in $M e C P 2$ (Amir et al., 1999), which resides on the X-chromosome. This was discovered in the lab of Huda Zoghbi by screening for mutations within $\mathrm{Xq} 28$, a region of the $\mathrm{X}$ chromosome that had been 
previously linked to Rett syndrome (Amir et al., 1999). Due to its X-linked nature, Rett syndrome patients are overwhelmingly female, because the relatively rare males with MeCP2 mutations (Jan et al., 1999) tend to suffer much more severe outcomes, with death typically occurring within the first year of life (Chahrour and Zoghbi, 2007). This can most likely be explained by genetic differences between males and females due to Xinactivation. Females inheriting $M e C P 2$ mutations will possess both normal and MeCP2mutant cells. Due to random inactivation of $\mathrm{X}$ chromosomes, each individual female cell will only express one allele of $M e C P 2$. Therefore, any given female cell will only express either the normal or mutant version of the protein, creating a situation where $M e C P 2$ normal and $M e C P 2$-mutant cells coexist in the same individual. However, males possess only one $\mathrm{X}$ chromosome. Thus, males with mutation of $\mathrm{MeCP} 2$ will express the mutant gene product in all cells, making the mutation fully penetrant throughout the body. This results in the most severe potential disease outcomes, including death within 1 to 3 years of age (Chahrour and Zoghbi, 2007).

Interestingly, it has been shown that $\mathrm{MeCP} 2$ expression increases during brain development in rodents, and correlates with neuronal maturation (Jung et al., 2003; Kishi and Macklis, 2004; Mullaney et al., 2004; Shahbazian et al., 2002). This finding has been corroborated in human samples as well (Balmer et al., 2003). This suggests that the early normal development observed in Rett syndrome patients may be explained by lower levels of MeCP2 expression in all female patients until about 6-18 months of age, when MeCP2 expression may normally increase and be required for development from that point forward. However, in patients with Rett syndrome, the lack of functional MeCP2 
expression when it would normally be required likely results in a failure to effectively continue healthy development, and pathology ensues.

Since the discovery that mutations in $M e C P 2$ are the major cause of Rett syndrome, over 300 different mutations have been identified (Christodoulou et al., 2003). This may help to explain the significant phenotypic variability observed in Rett syndrome patients, and in support of this concept, it has been shown that specific mutations are in fact predictive of disease outcome and severity (Cuddapah et al., 2014; Neul et al., 2014). This complicates the scientific study of Rett syndrome, because it suggests significant complexity in the function of $\mathrm{MeCP} 2$ that cannot be elucidated by simply deleting the protein in its entirety. Thus, it might be prudent to consider the study of MeCP2 and Rett syndrome as related, but not identical pursuits. However, as with many things in science, the most logical approach may be to first understand what MeCP2 does in its totality within a cell, with either concurrent or follow up studies employing such basic science knowledge to unravel the increased complexity present in the human disorder.

On top of the complexity introduced by the hundreds of $M e C P 2$ mutations, one must also consider the fact that the majority of patients, who are females, will contain within their tissues both cells expressing normal MeCP2 protein and other cells expressing mutant $\mathrm{MeCP} 2$ protein. Thus, the study of Rett syndrome in female patients is not only a study of the effects of MeCP2 mutation, but it is also a study of the interaction between MeCP2-normal and MeCP2-mutant cells in a single organism. In addition, the degree of $\mathrm{X}$ inactivation skewing can further influence disease outcomes by either increasing or decreasing the ratio of MeCP2-mutant to MeCP2-normal cells in the 
patient. These additional layers of complexity highlight the need to both study the basic functions of $\mathrm{MeCP} 2$ in a more "pure" system (such as complete removal or overexpression of the protein in all cells), and to simultaneously utilize this knowledge to inform studies of the more complex situation which exists in Rett syndrome patients.

\section{MeCP2 duplication syndrome}

While $\mathrm{MeCP} 2$ mutations were identified as the genetic cause of Rett syndrome in 1999, it was not until years later that duplication of the gene was found to also result in a neurodevelopmental disorder, now termed MeCP2 duplication syndrome (Van Esch et al., 2005). In more rare instances, triplication of $M e C P 2$ has been identified, resulting in a more severe form of the disease (del Gaudio et al., 2006). The exact genetic defect has been identified as a duplication of the $\mathrm{Xq} 28$ region of the $\mathrm{X}$ chromosome, which contains the $M e C P 2$ gene (del Gaudio et al., 2006; Friez et al., 2006; Meins et al., 2005; Ramocki et al., 2009; Ramocki et al., 2010; Sanlaville et al., 2005; Smyk et al., 2008; Van Esch et al., 2005).

A disorder due to $\mathrm{MeCP} 2$ gene overexpression was actually predicted based on mouse models prior to its discovery in humans, as overexpression of the human MeCP2 protein in mice resulted in neurologic abnormalities, including seizures, spasticity, kyphosis, and hypoactivity (Collins et al., 2004; Ramocki et al., 2010). Interestingly, these mice displayed increased motor learning and synaptic plasticity (Collins et al., 2004), although humans with MeCP2 duplication syndrome do not display any cognitive 
or learning advantages, with negative consequences of gene duplication dominating in patients (Ramocki et al., 2010).

Unlike Rett syndrome, MeCP2 duplication syndrome is typically found in males (Ramocki et al., 2010). However, similar to Rett syndrome, the X-linked nature of $M e C P 2$ explains the sex-based demographics of the disease. Following the same principles leading to more severe outcomes in males with $\mathrm{MeCP} 2$ mutations, males inheriting $M e C P 2$ duplication will express the duplicated $\mathrm{X}$ chromosome in all cells, due to the fact that males possess only one $\mathrm{X}$ chromosome. However, a curious dichotomy between mutation and duplication of $M e C P 2$ exists in the fact that females inheriting MeCP2 duplication have been shown to skew almost $100 \%$ towards the normal $\mathrm{X}$ chromosome (Ramocki et al., 2009; Ramocki et al., 2010). This means that, unlike $M e C P 2$ mutation and Rett syndrome, females with $M e C P 2$ duplication will not display severe symptoms, and thus will go through life unaware that they are carriers of a genetic abnormality capable of causing severe problems in their male offspring (Ramocki et al., 2009; Ramocki et al., 2010). On close examination, however, females with MeCP2 duplication are found to have more subtle abnormalities which are not specifically diagnostic for $M e C P 2$ duplication, such as depression, compulsivity, anxiety, and autistic features (Ramocki et al., 2009; Ramocki et al., 2010). An outstanding question remains as to why $M e C P 2$ duplication results in favorable $\mathrm{X}$ skewing, while $\mathrm{MeCP} 2$ mutation does not. The fact that $M e C P 2$ duplication induces such changes in $\mathrm{X}$ skewing suggests that intrinsic mechanisms for detecting abnormalities of $M e C P 2$ within the cell (and/or 
proximal genetic regions) may exist, and if understood might represent an avenue for therapeutic exploitation.

While the study by Ramocki et al. demonstrated overall favorable X-skewing in females with $M e C P 2$ gene duplications (Ramocki et al., 2009), more recent case studies have identified females with symptomatic MeCP2 duplication syndrome and either random X skewing (Grasshoff et al., 2011; Makrythanasis et al., 2010; Mayo et al., 2011; Shimada et al., 2013a), or in one case, an unfavorable skewing pattern (a ratio of 12:88 in one case, with the $\mathrm{X}$ chromosome containing the duplication dominating) (Shimada et al., 2013b). Interestingly, in the case of the female with an unfavorable skewing pattern, the mother was also assessed for $\mathrm{X}$ inactivation, and it was found that she had a random $\mathrm{X}$ inactivation pattern, but did not display disease phenotype (Shimada et al., 2013b). Although it is still assumed that most females will be asymptomatic carriers due to favorable $\mathrm{X}$ skewing, these cases highlight the fact that in some instances, symptomatic females with MeCP2 duplication syndrome will occur, and therefore such a potential diagnosis should not be automatically ruled out.

The clinical features of $\mathrm{MeCP} 2$ duplication syndrome somewhat mirror those of Rett syndrome, however there are several distinguishing features that change the focus of treatment for these patients. MeCP2 duplication syndrome, like Rett syndrome, will result in epileptic seizures, motor dysfunction including hypotonia and ataxia, growth deficiencies including microcephaly, bruxism, and speech impairment may all be present (Chahrour and Zoghbi, 2007; Friez et al., 2006; Ramocki et al., 2009; Ramocki et al., 
2010; Van Esch et al., 2005). MeCP2 duplication patients are also characterized by progressive spasticity, facial dysmorphia, and severe mental retardation.

Unlike Rett syndrome, patients with MeCP2 duplication syndrome experience significant mortality, with almost $40 \%$ of patients dying before the age of 25 (Ramocki et al., 2010). The major cause of mortality is recurrent respiratory infections, although patients will also experience ear and sinus infections, with an overall incidence of recurrent infection in 75\% of patients (Friez et al., 2006; Ramocki et al., 2010; Van Esch et al., 2005). This differentiates MeCP2 duplication syndrome from Rett syndrome, as no known susceptibility to infection occurs in Rett and the risk of mortality is substantially less (Chahrour and Zoghbi, 2007). While recurrent infections, especially in the respiratory tract, represent a major cause of morbidity and mortality, few scientific investigations have been performed towards understanding the etiology of this aspect of disease.

Neurologic failures may contribute to infections via esophageal dysfunction (which may lead to aspiration pneumonia). However, several groups have identified primary defects of the immune response in the context of MeCP2 overexpression. In mice and patients, it was shown that T helper 1 (Th1) skewed $\mathrm{T}$ cells, which are important for clearance of many pathogens, are deficient in production of an important canonical cytokine, interferon gamma (IFN $\gamma$ ) (Yang et al., 2012). This was postulated to explain a failure to clear infections in patients. Another group observed that MeCP2 overexpressing mice naturally develop anti-nuclear antibodies, which are associated with autoimmune disease (Koelsch et al., 2013). An analysis of the immune status in patients did not 
measure any baseline $\mathrm{T}$ cell abnormalities, including a finding of normal IFN $\gamma$ production, however they did measure significant abnormalities of antibody production and an excessive acute phase response in the context of infection (Bauer et al., 2015). Interestingly multiple groups have identified antibody abnormalities, including a deficiency of immunoglobulin A (IgA) (Bauer et al., 2015; Friez et al., 2006). This may be an important finding, as the infections seen in patients all share in common a mucosal site (respiratory tract, ear, sinus), and IgA is central to mucosal immunity (Macpherson et al., 2008). Additional work is needed to further understand the picture of immune function in the context of $\mathrm{MeCP} 2$ duplication. Given the fact that respiratory infections are the major cause of death (Ramocki et al., 2010), infection control represents one of the most pressing unmet needs of patients. A better mechanistic understanding of the immune failures leading to recurrent infection in patients may realistically lead to treatments, which will greatly improve lifespan and quality of life.

\section{Mouse models of Rett syndrome and the role of MeCP2 in neurons}

From the initial discovery that mutations in $\mathrm{MeCP} 2$ lead to Rett syndrome (Amir et al., 1999), it was expected that the protein would play a critical role in neuronal function. This was a logical assumption due to the severe neurologic sequelae inherent to Rett syndrome, as previously discussed. Mecp2-null mouse models were developed in the labs of Rudolph Jaenisch and Adrian Bird, two prominent figures in the study of methyl-CpG binding proteins, with the latter having discovered MeCP2 nearly a decade earlier (Lewis et al., 1992; Meehan et al., 1992). The features of both Mecp2-null mouse lines 
confirmed neurologic pathology is associated with deletion of Mecp2 in mice (Chen et al., 2001; Guy et al., 2001). Primary features of Mecp2-null mice included hindlimb clasping (thought to arise from central nervous system (CNS) impairment in mice), impaired gait, abnormal weight, tremors, breathing disturbances, tooth and jaw misalignment, and peripheral autonomic dysfunction including cold extremities (Chen et al., 2001; Guy et al., 2001). In both models, the Mecp2-null male mice died between 6-12 weeks of age, whereas Mecp2-heterozygous female mice lived several months prior to the onset of comparatively mild symptoms, and did not exhibit early mortality. The severe outcome in Mecp2-null male mice as compared to Mecp2-heterozygous female mice agreed with findings in humans with $\mathrm{MeCP} 2$ loss-of-function mutations, because males generally die very young and have a much more severe form of the disease, as previously discussed (Chahrour and Zoghbi, 2007).

A curious finding was the fact that in the Mecp2-null model developed in the Bird lab, animals were assessed on both a pure C57BL6 and C57BL6/129 F1 background. On the C57BL6 background, the animals were smaller than their wild type littermates, with an average peak weight between $10-15 \mathrm{~g}$ (versus $\geq 20 \mathrm{~g}$ for wild type littermates). However, Mecp2-null mice on the C57BL6/129 F1 background were the same size as wild type littermates initially, and even began to exceed the weight of wild type mice due to increased fat deposition (Guy et al., 2001). This raises an important aspect of working with a complex epigenetic regulator, because although $\mathrm{MeCP} 2$ clearly plays a role in growth and development, the exact outcome of Mecp2 perturbation in mice may depend heavily upon the genetic background strain selected, since the core function of MeCP2 is 
to regulate the expression of a plethora of other genes (Chahrour et al., 2008; Tudor et al., 2002). Thus, epigenetic or allelic differences between different strains of mice may significantly alter how Mecp2 interacts with any other number of transcriptional targets, resulting in apparently opposing phenotypes, as seen in this example. There may be similar background strain-dependent phenotypes, many of which researchers are completely unaware of, littered throughout the $\mathrm{MeCP} 2$ literature, and thus the proper reporting and consideration of background strain is absolutely essential for the responsible conduct of research in this arena to avoid confusion over such apparent inconsistencies.

In the original studies, both groups reported that Nestin $^{C r e}$ mediated excision of Mecp2 resulted in similar disease phenotype to Mecp2-null animals (Chen et al., 2001; Guy et al., 2001). These experiments were taken as evidence that neurons are the primary drivers of pathology, although it was acknowledged that Nestin is in fact expressed in glial cells as well (Guy et al., 2001). No survival data was presented for Nestin ${ }^{\text {Cre }}$ mice, so it is unclear whether lifespan was comparable to Mecp2-null. In the study from Jaenisch's lab, a second cre thought to be more specific to neurons was used, $\operatorname{CamK}^{\mathrm{Cre} 93}$. This resulted in a milder, delayed form of disease, with animals remaining healthy for up to three months, a time point when Mecp2-null mice have all died (Chen et al., 2001). However, between the Nestin ${ }^{C r e}$ and $\mathrm{CamK}^{\mathrm{Cre} 93}$ models of Mecp2 deletion, a conclusion was drawn that disease caused by Mecp2-deficiency in mice is due to loss of Mecp2 in neurons, and not glia or other somatic cell types (Chen et al., 2001). 
Importantly, it has since been found that key populations of cells in the periphery also express nestin, such as mesenchymal stem cells in the bone marrow (Mendez-Ferrer et al., 2010), thus conclusions that the effects of Nestin ${ }^{C r e}$ are solely due to loss of Mecp2 in neurons and glia can no longer be considered valid. When this is coupled with the fact that the more neuron-specific cre, $\operatorname{Cam}^{\mathrm{Cr} e 93}$, resulted in a much milder form of disease, the neuron-only model of disease begins to appear overly simplistic. Indeed, while some models were able to ameliorate symptoms in otherwise Mecp2-null mice via expression of Mecp2 under Tau, Nestin, or, CamKII promoters (Giacometti et al., 2007; Luikenhuis et al., 2004), another group was unable to recapitulate any benefit from Mecp2 expression under the CamKII promoter, which was confirmed to result in widespread Mecp2 expression throughout the forebrain, or the neuron-specific Eno2 promoter, which was confirmed to result in Mecp2 expression throughout the striatum, hippocampus, superior colliculus, and cerebellum (Alvarez-Saavedra et al., 2007). These conflicting studies bring into question whether or not disease is truly driven by Mecp2 deficiency in neurons alone, or if an experimental artifact, such as unexpected expression of Mecp2 in cells that were not interrogated in specific studies, may point to a more complex etiology of disease.

Regardless as to whether or not disease is caused by Mecp2-deficiency in neurons alone, a large body of literature has supported the importance of MeCP2 in neurons of many subtypes (Guy et al., 2011). It was reported in the original study of Mecp2-null mice from Jaenisch's lab that the overall brain size and neuronal soma size was reduced in Mecp2-null mice (Chen et al., 2001). Since then, many cre-mediated approaches have 
removed the protein from specific neuronal subtypes, and have found limited deficits throughout the brain (Guy et al., 2011). Loss of Mecp2 from aminergic neurons in mice using $T h^{C r e}$ or Pet $1^{C r e}$ resulted in no change in lifespan, but changes in activity level (hypo- or hyperactivity, respectively), increased aggression in Pet1 $^{\text {Cre }}$ conditional knockouts, and an overall reduction in neurotransmitter production by the targeted neuronal subtypes in each model were observed (Samaco et al., 2009). Sim1 $1^{\text {Cre }}$ conditional Mecp2 knockout in hypothalamic neurons resulted in normal life span with enhanced physiologic response to stress, hyperphagia and obesity accompanied by increased levels of leptin in later life, and increased aggression (Fyffe et al., 2008). Importantly, these mice were bred to a 129/FVB F1 background, which as previously discussed (Guy et al., 2001), may have significantly affected the obesity phenotype observed in $\operatorname{Sim} 1^{\text {Cre }}$ conditional Mecp2 knockout mice. Conditional knockout was also performed in parvalbumin and somatostatin-positive neurons, also resulting in partial disease phenotypes (Ito-Ishida et al., 2015). As previously mentioned, CamKII' mediated deletion of Mecp2 results in mild phenotypes compared to Mecp2-null animals (Chen et al., 2001), and further characterization revealed that specific deficits include hindlimb clasping, impaired coordination, increased anxiety, and impaired social behavior with relatively normal lifespans (Gemelli et al., 2006).

Viaat-mediated deletion of Mecp2 from GABAergic neurons resulted in reduction of $\mathrm{Gad} 1, \mathrm{Gad} 2$, and GABA expression in those neurons, with reduced survival ( $\sim 50 \%$ by 26 weeks), repetitive behavior, self-injury, coordination impairment, progressive hypoactivity, increased social behavior, and breathing dysfunction (Chao et al., 2010). In 
the same study, Dlx5/6-mediated Mecp2 deletion in forebrain GABAergic neurons alone resulted in some of the functional impairments, but did not affect lifespan. This was interpreted to indicate that hindbrain GABAergic neurons targeted in the Viaat ${ }^{\text {Cre }}$ conditional Mecp2 knockout may drive mortality, specifically through respiratory dysfunction (Chao et al., 2010), however the possibility still exists that some off-target deletion of Mecp2 in Viaat $^{C r e}$ mice may contribute to differences with Dlx5/6-mediated excision.

In addition to cre-mediated manipulation of Mecp 2 expression in mice in vivo, specific neuronal functional defects have also been measured. Synaptic plasticity has been implicated as a major altered function in either the case of Mecp2-deletion (Asaka et al., 2006), or overexpression (Collins et al., 2004). In the case of Mecp2-null mice, hippocampal long-term potentiation (Guy et al., 2007; Weng et al., 2011) and long-term depression were impaired in an age and disease-dependent manner (Asaka et al., 2006). Reduced long-term potentiation was also observed in another mouse model of Rett syndrome in which a human loss-of-function mutation was inserted into mice $\left(\right.$ Mecp $\left.^{308 / y}\right)$ (Moretti et al., 2006). MeCP2 also directly regulates the number of glutamatergic synapses in hippocampal neurons (Chao et al., 2007). In this study, Mecp2null neurons were shown to have an almost $50 \%$ reduction in their excitatory synaptic response, while $\mathrm{MeCP} 2^{\mathrm{Tg} l}$ neurons, which have an inserted human $\mathrm{MeCP} 2$ gene resulting in approximately two-fold overexpression of $\mathrm{MeCP} 2$, displayed a two-fold increase in synaptic response (Chao et al., 2007). This reciprocal relationship between glutamatergic 
synapse number, and consequential change in synaptic response, gives strong evidence that $\mathrm{MeCP} 2$ directly regulates glutamatergic synapse number in hippocampal neurons.

Together, these studies demonstrate that $\mathrm{MeCP} 2$ is required for healthy neuronal function. MeCP2 appears to regulate several functions, including neurotransmitter production, synaptic plasticity, and excitatory/inhibitory balance (Dani et al., 2005; Guy et al., 2011; Nelson et al., 2006). Importantly, loss of MeCP2, and thus Rett syndrome, is not a neurodegenerative process (Chahrour and Zoghbi, 2007), and thus significant neuron loss is not a feature of the disease.

Regardless as to the specific role of $\mathrm{MeCP} 2$ in neurons, and the overall importance of neuronal $\mathrm{MeCP} 2$ in the etiology of disease, an important study demonstrated that postnatal re-expression of Mecp2 in all cells throughout the body can mediate significant symptomatic improvement and survival in mice (Guy et al., 2007). Even mice that were already symptomatic showed improvement. Therefore, in theory, disease caused by $\mathrm{MeCP} 2$ deficiency, and thus Rett syndrome, may be at least partially reversible. Conversely, it was shown that Mecp2 removal from adult mice results in development of symptoms and reduced survival, remarkably similar to Mecp2-null mice (McGraw et al., 2011). Based on these studies, the important conclusion can be made that re-expression or deletion of Mecp2 in adulthood can either ameliorate or induce symptoms caused by loss of Mecp2. Therefore, recent work has focused on genetic therapy (Garg et al., 2013) or control of X chromosome inactivation (Bhatnagar et al., 2014) in order to correct MeCP2 levels postnatally in Rett syndrome patients. 


\section{The role of $\mathrm{MeCP} 2$ in astrocytes and oligodendrocytes}

Although the importance of MeCP2 in neurons is clear, its role in other cell types has only become a subject of scientific inquiry within the last decade. This is in large part because early antibodies against $\mathrm{MeCP} 2$ were unable to detect $\mathrm{MeCP} 2$ in non-neuronal cell types due to their lower level of MeCP2 expression (Guy et al., 2011). However, a study from the lab of Gail Mandel in 2009 using a novel antibody against Mecp2 was able to detect the protein in mouse glia, although it was expressed at significantly lower levels than in neurons (Ballas et al., 2009). In this study, Mecp2 was detected in all glial subtypes, including astrocytes, oligodendrocyte precursor cells, oligodendrocytes, and microglia, although the data for microglia was not shown. Later publications have recapitulated these findings in astrocytes (Maezawa et al., 2009; Song et al., 2014), oligodendrocytes (Nguyen et al., 2013), and microglia (Cronk et al., 2015a; Maezawa and Jin, 2010).

In the first study where Mecp2 expression was demonstrated in mouse glia, it was also demonstrated that Mecp2-deficient glia contribute to pathology (Ballas et al., 2009). The authors showed that neither Mecp2-null astrocytes nor their conditioned media were able to support neuronal growth in vitro, and inversely, wild type astrocytes and their conditioned media were able to rescue dendritic morphology of Mecp2-null neurons (Ballas et al., 2009). This was the first evidence that mechanisms outside cellautonomous impairment of neurons may account for symptoms of Rett syndrome.

Another study in the same year also identified detrimental consequences of Mecp2 deficiency in astrocytes (Maezawa et al., 2009). Here, it was shown that Mecp2- 
deficient astrocytes are impaired in brain-derived neurotrophic factor (BDNF) production, cytokine production, and the failure of Mecp2-deficient astrocytes to support normal neuronal dendritic growth was repeated (Maezawa et al., 2009). In addition, the claim was made that Mecp2-deficient astrocytes actually spread Mecp2 deficiency via gap junctions. This claim was not substantiated by direct measurement of Mecp2 protein transfer, but rather by pharmacologic blockade of gap junctions and siRNA treatment against a key gap junction component, connexin 43. No groups have reported on such phenomena since, but it is an intriguing possibility that Mecp2-deficient astrocytes may in fact spread disease directly to other cells (Maezawa et al., 2009).

The failure of Mecp2-deficient astrocytes to support neuronal dendrites in mice was also recapitulated using induced pluripotent stem cell (iPSC)-derived astrocytes from human Rett syndrome patients (Williams et al., 2014), thus providing yet more support for the role of astrocytes in neuronal dendritic pathology in Rett syndrome. Interestingly, this study also found that insulin-like growth factor 1 (IGF-1) treatment was able to correct neuronal dendritic deficiencies in the context of Rett syndrome iPSC-derived astrocytes (Williams et al., 2014). This is of particular interest, as it was previously shown that IGF-1 treatment significantly ameliorated disease in both Mecp2-null male and Mecp2-heterozygous female mice (Castro et al., 2014; Tropea et al., 2009). Clinical trials of IGF-1 in Rett syndrome patients are promising, demonstrating no negative effects (Pini et al., 2012), and early data suggests improvements in behavior and breathing parameters (Khwaja et al., 2014), although additional subjects in the next phases of clinical trial will be needed to confirm these findings. 
Multiple groups have performed transcriptome-level analysis of astrocytes from mouse models of Rett syndrome, finding significant changes in gene expression (Delepine et al., 2015; Yasui et al., 2013), and confirming Mecp2 binding to the promoters of predicted target genes via chromatin immunoprecipitation (ChIP)-seq (Yasui et al., 2013). It was also demonstrated that Mecp2-null astrocytes have abnormal glutamate clearance (Okabe et al., 2012), a finding that has also been observed in Mecp2null microglia (Maezawa and Jin, 2010), and which may have direct effects on neurons.

Possibly the most important study regarding astrocytes came in 2011, when it was demonstrated that postnatal-inducible expression of Mecp2 in astrocytes using a glial fibrillary acidic protein (GFAP) promoter was able to not only improve symptoms, but also significantly increased lifespan of otherwise Mecp2-deficient mice (Lioy et al., 2011). Behavioral outcomes were improved, including locomotion, anxiety, and breathing. In addition, cellular defects predicted to be impaired by Mecp2-deficient astrocytes (Ballas et al., 2009; Maezawa et al., 2009; Williams et al., 2014) were also improved, including increased dendritic branching, increased neuronal soma size, and VGLUT1 expression was increased in neurons (Lioy et al., 2011). It is important to point out that when the reverse experiment was performed, where Mecp2 was specifically removed from astrocytes, very little pathology was observed (Lioy et al., 2011). This suggests that astrocytes are either not the major driver of disease, or that Mecp2 must be deficient in multiple cell types in order for significant disease to be observed. This is a finding that has been noted in many other models when Mecp2 was only removed from individual cell populations, or specific neuronal subtypes (as previously discussed), 
suggesting that the disease resulting from whole-organism MeCP2 deficiency (i.e. Rett syndrome) is the result of pathology in multiple cell types, and cannot likely be explained by simplistic models equating to a "one molecule, one cell, one disease" hypothesis.

More recently, the role of $\mathrm{MeCP} 2$ in oligodendrocytes was assessed by either removing or re-expressing Mecp2 in otherwise Mecp2-null mice (Nguyen et al., 2013). While the effects were overall mild, it was observed that removal of Mecp2 from oligodendrocytes alone resulted in the hindlimb clasping phenotype classically observed in Mecp2-null mice (Chen et al., 2001; Guy et al., 2001), suggesting that Mecp2 deficiency in oligodendrocytes may be the causative factor behind this phenotype. When Mecp2 was restored in the oligodendrocytes of otherwise Mecp2-null animals, pathology observed upon Mecp2 removal from oligodendrocytes, such as hindlimb clasping, was improved, and lifespan was mildy increased (approximately 3 weeks) (Nguyen et al., 2013). It is important to mention, however, that the cre used in this study was under the promoter for the gene encoding neuron-glial antigen $2(\mathrm{Ng} 2)$, which is also expressed by other cells, such as some grey matter astrocytes (Zhu et al., 2008a; Zhu et al., 2008b), and pericytes (Armulik et al., 2011). Therefore, it is possible that the effects seen in this study were due to the expression of Mecp2 in other cells types, or some combination of Mecp2 expression in multiple cell types. However, MeCP2 was also shown to regulate transcription of myelin genes in rats, providing evidence of a potential mechanism for MeCP2 in oligodendrocyte function (Sharma et al., 2015). 


\section{MeCP2 and microglia}

The first immune cell to be implicated in Rett syndrome was microglia (Maezawa and Jin, 2010). We, and others, have demonstrated that Mecp2 is expressed by and functionally important for microglia (Cronk et al., 2015a; Derecki et al., 2012; Horiuchi et al., 2016; Jin et al., 2015; Maezawa and Jin, 2010).

Microglia are the resident immune cells of the brain, which have garnered significant attention from neuroscientists and immunologists alike in recent years (Prinz and Priller, 2014). Microglia are now thought to be important for not only immune responses upon injury or infectious insult, but also play important roles in brain homeostatic maintenance (Cronk and Kipnis, 2013). Specifically, it has been shown that microglia are involved in synaptic pruning (Bialas and Stevens, 2013; Ji et al., 2013; Paolicelli et al., 2011; Schafer et al., 2012), synaptic stripping (Trapp et al., 2007), neuronal precursor growth and numbers during brain development (Cunningham et al., 2013; Ueno et al., 2013), regulation of neural progenitor cells (NPCs) via both phagocytosis of apoptotic NPCs and production of growth factors to regulate their development (Butovsky et al., 2006; Sierra et al., 2010), and promotion of learningdependent synapse formation via BDNF (Parkhurst et al., 2013). Based on these findings, the idea that microglia may in fact be important players in neurologic and psychiatric disease has gained traction (Prinz and Priller, 2014).

The first evidence that microglia are impaired in the absence of Mecp2 came in 2010, when it was shown that Mecp2-null microglia produce excessive levels of glutamate, resulting in neuronal synapse and dendrite damage (Maezawa and Jin, 2010). 
Another group later recapitulated the finding of enhanced glutamate release in human peripheral blood mononuclear cells (PBMC), another myeloid cell type (O'Driscoll et al., 2013b). Interestingly, it was also shown that Mecp2-null astrocytes produce excessive glutamate, raising the possibility that $\mathrm{MeCP} 2$ may play a more global role in glutamate metabolism (Okabe et al., 2012). The same group of scientists that originally described enhanced glutamate release by Mecp2-null microglia later provided mechanistic evidence to explain this finding in 2015 (Jin et al., 2015). In this follow up publication, it was demonstrated that Mecp2 is a transcriptional repressor of sodium-coupled neutral amino acid transporter 1 (Snat1), a glutamine transporter. Thus, in the absence of Mecp2, Snat1 was overexpressed, resulting in excessive glutamate production via dysregulation of glutamine transport. This also resulted in proliferation of mitochondria and mitochondrial reactive oxygen species (ROS) production, which was accompanied by increased oxygen consumption and reduced production of ATP (Jin et al., 2015). The authors also postulated that in addition to neuronal damage, microglia were also lost in Mecp2-null brains due to Snat1 overexpression and the subsequent metabolic defects (Jin et al., 2015).

In the most recent publication from the same group, chemokine ( $\mathrm{C}-\mathrm{X} 3-\mathrm{C}$ motif) receptor 1 (Cx3cr1) was deleted in the Mecp2-null mouse (Horiuchi et al., 2016). Cx3cr1 is an important chemokine receptor expressed highly on microglia (Jung et al., 2000), thus it is not surprising that its manipulation may alter microglial function. Deletion of Cx3cr1 in Mecp2-null mice resulted in improvement of symptoms, including increased weight, improved neurologic scores, normalized breathing, and a moderate increase in 
survival. Importantly, the authors also found that $\mathrm{Cx} 3 \mathrm{cr} 1$ deletion in Mecp2-null mice normalized microglia numbers (Horiuchi et al., 2016), suggesting a role for $\mathrm{Cx} 3 \mathrm{cr} 1$ or downstream signaling in mediating microglia loss that had been previously observed in Mecp2-null mice (Cronk et al., 2015a; Jin et al., 2015). As a potential explanation for improvement in Cx3cr1-null / Mecp2-null mice, the authors found that Igf1 expression was increased (Horiuchi et al., 2016), a molecule which had already been shown to mediate benefit in both mice (Castro et al., 2014; Tropea et al., 2009) and potentially human Rett syndrome patients (Khwaja et al., 2014; Pini et al., 2012).

In sum, the literature of the past several years has revealed that $\mathrm{MeCP} 2$ is expressed by and important for all glia, including microglia, astrocytes, and oligodendrocytes. These works demonstrating an important role for $\mathrm{MeCP} 2$ in glia fundamentally changed the scientific approach to $\mathrm{MeCP} 2$ and its related disorders, as it had been previously assumed that MeCP2 was, essentially, a neuronal protein. The discovery of MeCP2 in glia was quickly followed by the study of MeCP2 in other cell types entirely outside of the CNS. Roles for Mecp2 in skeletal muscle (Gold et al., 2014), cardiac and skeletal tissue during development (Alvarez-Saavedra et al., 2010), osteoblasts (Blue et al., 2015), and cholesterol metabolism (Buchovecky et al., 2013) have been demonstrated. Importantly, MeCP2 is also expressed by and required for the proper function of many immune cells (Cronk et al., 2015b), as will be discussed in the next section. 


\section{MeCP2 in adaptive immunity}

The study of MeCP2 in the immune system has only become a topic of interest within the last several years, again likely because of the heavy focus on the role of MeCP2 in neurons following the discovery that mutations in $\mathrm{MeCP} 2$ cause Rett syndrome. However, evidence suggests that $\mathrm{MeCP} 2$ is not only related to Rett syndrome and MeCP2 duplication syndrome, but may also contribute to primary immunologic disorders, such as systemic lupus erythematosus (SLE) (Kaufman et al., 2013; Koelsch et al., 2013; Sawalha et al., 2008; Webb et al., 2009), primary Sjogren's syndrome (Cobb et al., 2010), systemic sclerosis (Carmona et al., 2013), and rheumatoid arthritis (Han et al., 2013). Importantly, all of these diseases are autoimmune disorders, which are caused by improper targeting of the adaptive immune system against self. This association of $\mathrm{MeCP} 2$ allelic variants with autoimmune disorders points towards a direct role for MeCP2 in the development of autoimmune disease. Indeed, it was found that overexpression of $\mathrm{MeCP} 2$ in mice leads to development of autoantibodies against nuclear antigens (Koelsch et al., 2013), which are a prominent feature of SLE (Lerner and Steitz, 1979). These non-biased, independent genetic studies by separate groups identifying an association between $\mathrm{MeCP} 2$ variants and autoimmune disease provide some of the strongest evidence that $\mathrm{MeCP} 2$ is in fact important for cells other than neurons in human disease, and that the study of MeCP2 and its functions should be considered outside of the brain. Moreover, functional roles for $\mathrm{MeCP} 2$ should be considered outside of the context of Rett and MeCP2 duplication syndromes. 
One of the cell types thought to play a critical role in autoimmune disease is $\mathrm{T}$ cells, especially through regulatory $\mathrm{T}$ cells (Treg) which are potent suppressors of autoreactive effector T cells (Sakaguchi et al., 2010). One clue as to how MeCP2 may play a role in autoimmune disease came from a recent study in which it was shown that MeCP2 is required to maintain forkhead box P3 (Foxp3) expression in Tregs (Li et al., 2014). Foxp3 is a critical transcription factor for the suppressive function of Tregs (Wu et al., 2006), and is one of the canonical markers used to identify these cells. In this work, the authors demonstrated that in the context of Treg-specific deletion of Mecp2 in mice, Tregs lose Foxp3 expression over time, are unable to control effector T cell-mediated colitis, and develop spontaneous immune activation ( $\mathrm{Li}$ et al., 2014). They further showed that Mecp2 binds to the Foxp3 genetic locus, and directly controls its expression (Li et al., 2014).

Other groups have also investigated the role of MeCP2 in T cells. A very early study in lymphocytes from Rett syndrome patients demonstrated that MeCP2-mutant T cells were underrepresented and had a growth disadvantage after mitogenic stimulation in vitro (Balmer et al., 2002). More recent work has demonstrated a role for Mecp2 in T cell skewing and cytokine production in mice (Jiang et al., 2014). More specifically, Mecp2 was shown to be critical for T helper 17 (Th17) and Th1 skewing, and IFN $\gamma$ production by Th1 T cells. This was mediated by a loss of miR-124 in Mecp2-null CD4+ T cells. Loss of miR-124 led to an accumulation of suppressor of cytokine signaling 5 (Socs5), which in turn inhibited signal transducer and activator of transcription 1 (Stat1) and 
signal transducer and activator of transcription 3 (Stat3), which are critical for Th1 and Th17 skewing and cytokine production, respectively (Jiang et al., 2014).

As was previously discussed within the section entitled "MeCP2 duplication syndrome," impaired IFN $\gamma$ production was observed in Th1 CD4+ T cells in both mice and humans with $\mathrm{MeCP} 2$ overexpression/duplication syndrome, respectively (Yang et al., 2012). Due to the prominent infection component present in MeCP2 duplication syndrome (Ramocki et al., 2010), deficiency of IFN $\gamma$ was postulated to explain susceptibility to infection in these patients (Yang et al., 2012). These findings agree with the concept that $\mathrm{MeCP} 2$ is critical for $\mathrm{T}$ cell skewing and cytokine production (Jiang et al., 2014), and provides a second point of evidence that $\mathrm{MeCP} 2$ is directly involved in IFN $\gamma$ production. As was also previously mentioned, a recent study of the immune status in $\mathrm{MeCP} 2$ duplication patients did not find evidence of $\mathrm{T}$ cell deficits, but rather implicated impairment of abnormal antibody isotype production and elevated acute phase responses upon infection (Bauer et al., 2015). Specifically, deficiency of $\operatorname{IgA}$ and immunoglobulin G (IgG) subtype $\operatorname{IgG} 2$ was observed, while $\operatorname{IgG} 1$ and $\operatorname{IgG} 3$ were elevated, suggesting that antibody class switching may be impaired in patients with MeCP2 duplication syndrome. Although the exact immune deficits leading to chronic infections in MeCP2 duplication patients remain unresolved, it is clear that MeCP2 overexpression leads to significant abnormalities of immune responses. The current immunologic evidence in the context of $\mathrm{MeCP} 2$ overexpression warrants further investigation to better understand the etiology of immune disease in these patients. 


\section{Thesis rationale}

Subsequent to the first published work demonstrating a role for MeCP2 in microglia (Maezawa and Jin, 2010), our group demonstrated that bone marrow transplant mediated significant symptomatic and survival improvement in Mecp2-null mice (Derecki et al., 2012). We concluded that engraftment of wild type microglia-like cells into the brains of Mecp2-null mice after bone marrow transplant accounted for the majority of benefits. At the time, it was thought that the cells engrafting into the brain after bone marrow transplant were essentially microglia; however, based on the discovery of unique microglial markers since publication of this work, it is now becoming clear that the "microglia-like cells" taking up residence in the brain after bone marrow transplant are in fact unique engrafting macrophages, separate from microglia (Butovsky et al., 2014; Waisman et al., 2015). This gives a new perspective on the benefits mediated by bone marrow transplant in Mecp2-null mice. It may be the case that some novel function of unique engrafting CNS macrophages after bone marrow transplant may account for the observed benefits, as opposed to restoration of endogenous microglial functions via replacement of true microglia by peripheral bone marrow-derived cells.

We also observed a significant improvement in survival and disease phenotypes when Mecp $2^{\text {LoxStop/y }}$ mice were crossed to Lysm ${ }^{\text {Cre }}$, resulting in Mecp2 re-expression in only Lysm-expressing cells in an otherwise Mecp2-null mouse (Derecki et al., 2012). Lysm $^{\text {Cre }}$ targets only a fraction of microglia, and actually recombines peripheral myeloid cells with greater efficiency (Clausen et al., 1999; Prinz and Priller, 2014), thus it is possible that peripheral myeloid cells may have played a larger role in disease 
amelioration than was previously recognized. In the context of bone marrow transplant, the peripheral immune system, including peripheral myeloid cells, were also replaced by wild type cells. The fact that both $L y s m^{C r e} / M e c p 2^{\text {LoxStop/y }}$ and wild type bone marrow transplant into Mecp2-null mice both result in significant genetic correction of Mecp2 expression in peripheral myeloid cells led us to consider their possible contribution to disease in Mecp2-null mice. Further, we did not explore the transcriptional role of Mecp2 in microglia in our original work (Derecki et al., 2012).

Given these unanswered questions, we decided to study both the functional role of Mecp2 in microglia and macrophages, and to examine the effects of Mecp2 deficiency on both microglia and peripheral macrophages during disease progression. The resulting work was published in the journal Immunity under the title "Methyl-CpG binding protein 2 regulates microglia and macrophage gene expression in response to inflammatory stimuli" (Cronk et al., 2015a). In this study, we found that Mecp2 is an important regulator of macrophage transcriptional response to multiple stimuli, including glucocorticoids, hypoxia, and inflammatory stimulation. In addition, we show that Mecp2 is expressed in a multitude of macrophage populations throughout the body, and that Mecp2-null mice lose microglia, macrophages, and resident monocytes. Finally, we demonstrate that Mecp2-null mice develop neutrophilia due to overproduction of granulocyte-colony stimulating factor (Gcsf), and that neutralization of Gcsf both corrects neutrophilia and mildly increases lifespan of Mecp2-null mice (Cronk et al., 2015a). This work is presented as a major focus of this dissertation in Chapter 3, and the original publication can be found in the Appendix. 
In the second part of this dissertation, we investigated the etiology of respiratory infection in the context of MeCP2 overexpression. As was previously discussed, MeCP2 duplication syndrome patients frequently succumb to respiratory infections. This, combined with the fact that very little literature exists examining this prominent cause of morbidity and mortality in patients, led us to develop a model of respiratory infection in MeCP2 overexpressing mice. In sum, we found that MeCP2 overexpressing mice are highly susceptible to influenza A/PR/8/34 infection, and experience complex pathology during infection, including excessive innate inflammatory responses, defective adaptive immunity, and a prominent role for non-hematopoietic cells in mortality. These findings are presented in Chapter 4.

In sum, the work presented in this dissertation provides multiple points of evidence that $\mathrm{MeCP} 2$ is an important player in immune function, both in the context of homeostasis and infection. Further, these cumulative findings suggest that many cell types express and require $\mathrm{MeCP} 2$ for their proper function within the immune system. Therefore, MeCP2 should be considered an essential immune molecule, and its role in immune interactions should continue to be a focus of future research. 


\section{Chapter 2}

\section{Materials and methods}

*NOTE: Materials and methods for experiments described in Chapter 3 can be found in the Appendix*

\section{Mice}

FVB-Tg(MECP2)3Hzo/J, B6.Cg-Tg(Nes-cre)1Kln/J, C57BL/6-Tg(UBC-GFP)30Scha/J, B6.129P2(C)Mecp2tm1.1Bird/J, B6.129P2-Mecp2tm2Bird/J, C57BL/6J (Jackson Laboratories) and $\mathrm{B} 6.129 \mathrm{~S} 7-\operatorname{Rag} 1^{\text {tmlMom }} \quad \mathrm{Tg}(\mathrm{TcraTcrb}) 1100 \mathrm{Mjb} \quad$ (Taconic) were maintained by in-house breeding. For experiments involving Mecp2-null mice (Chapter 3), mice were maintained on a pure C57BL6/J background. The background strain for all mice used in influenza infection experiments (Chapter 4) was first-generation F1 C57BL6 $x$ FVB (MeCP2 ${ }^{\mathrm{Tg} 3}$ female on FVB background bred to C57BL6 background male) to allow for use of transgenes and materials only available on and for the C57BL6 
background. All experiments and procedures complied with rules and regulations of the Institutional Animal Care and Use Committee at the University of Virginia.

\section{Mecp2 staining by flow cytometry in Mecp2-heterozygous female mice}

The identical protocol previously described (Cronk et al., 2015a) was used, and can be found in this dissertation in the Appendix.

\section{Mcsf injection into Mecp2-null mice}

Mecp2-null mice were either injected with vehicle (saline) or Mcsf, carrier-free (Biolegend) at $10 \mathrm{ng} / \mu \mathrm{l}$ concentration, $100 \mu \mathrm{g} / \mathrm{kg}$, intraperitoneally (i.p.). Injections were performed weekly starting at 4-5 weeks of age.

\section{Wild type monocyte transfer into Mecp2-null mice}

Wild type bone marrow monocytes were magnetically sorted using the Miltenyi monocyte isolation kit (BM), mouse per manufacturer instructions. 1.5-3 million monocytes or saline control were injected into Mecp2-null mice via tail vein weekly.

\section{Culture and in vitro stimulation of BMDM}

Bone marrow was isolated from femurs and cells were plated into macrophage media (DMEM/F12 media containing 10\% FBS, and Anti-Anti (Gibco)), and supplemented with $10 \mathrm{ng} / \mathrm{ml} \mathrm{Mcsf} \mathrm{(eBioscience)} \mathrm{at} \mathrm{a} \mathrm{density} \mathrm{of} 400 \mathrm{k}$ cells in $10 \mathrm{ml}$ media. Cells were grown in $100 \mathrm{~mm}$ non-TC treated petri dishes. After three days in culture, $5 \mathrm{ml}$ of 
macrophage media supplemented with $20 \mathrm{ng} / \mathrm{ml}$ Mcsf was added to each dish. On day 6 , mature macrophages were harvested by aspirating non-adherent cells, briefly washing with $5 \mathrm{ml}$ ice-cold PBS, aspirating, and then incubating with another $5 \mathrm{ml}$ ice-cold PBS for $5 \mathrm{~min}$. Cells were gently scraped with a rubber policeman, and harvested into $50 \mathrm{ml}$ conical tubes. Cells were spun down at $300 \mathrm{~g}, 10 \mathrm{~min}, 4^{\circ} \mathrm{C}$, resuspended in fresh macrophage media containing $10 \mathrm{ng} / \mathrm{ml}$ Mcsf, and plated at a density of 500k cells in each well of a non-TC treated 6 well plate. Cells were allowed to adhere overnight, and then were either treated with $50 \mathrm{ng} / \mathrm{ml}$ IFN $\gamma$ or media control for 6 hours. Cells were then treated with either $50 \mathrm{ng} / \mathrm{ml}$ LPS or media control for an additional 6 hours. At the conclusion of the experiment, RNA was harvested using Rneasy mini kit (Qiagen) per manufacturer instructions. qPCR was performed using Taqman reagents (Applied Biosystems). The Mecp2 probe used was Mm01193537_g1.

\section{Influenza infections}

Mice used for infection experiments were typically 12 weeks of age, with a range of 1015 weeks, and were age-matched within each experiment. Mice were anesthetized via intraperitoneal injection of ketamine/xylazine prior to intranasal infection with influenza $\mathrm{A} / \mathrm{PR} / 8 / 34$ in $50 \mu 1$ serum-free IMDM (Gibco). Unless otherwise specified, the standard dose used was $\mathrm{LD}_{100}$ for $\mathrm{MeCP} 2^{\mathrm{Tg} 3}$ mice, 3500 egg-infectious doses $\left(\mathrm{EID}_{50}\right)$. 


\section{Preparation of mouse blood for flow cytometry}

Blood samples for flow cytometry were collected after animal sacrifice via blood collection from the eye. Briefly, the eye was removed, and several drops of blood were collected into a heparinized tube to be used for whole blood cell count, and then the majority of blood was collected into heparin flow cytometry buffer containing heparin (1\% BSA, 2mM EDTA, $0.1 \%$ sodium azide, $10 \mathrm{u} / \mathrm{ml}$ heparin in PBS), centrifuged at $4^{\circ} \mathrm{C}$ for $10 \mathrm{~min}, 300 \mathrm{~g}$, supernatant was aspirated, and $5 \mathrm{ml} \mathrm{ACK}$ lysis buffer was used to lyse red blood cells by incubation for $5 \mathrm{~min}$, room temperature. ACK buffer was washed by addition of $45 \mathrm{ml} 4^{\circ} \mathrm{C}$ PBS, and centrifugation at $4^{\circ} \mathrm{C}$ for $10 \mathrm{~min}, 300 \mathrm{~g}$. Samples were then decanted and used for flow cytometry staining. Whole blood cell counts were obtained by 1:20 dilution of heparinized whole blood into acridine orange / propidium iodide staining solution in PBS and analysis on a Nexcelom Cellometer Auto 2000. Absolute blood cell counts were obtained by multiplication of the percentage of each population among total live cells measured via flow cytometry by the total live cell count.

\section{Preparation of lung tissue for flow cytometry}

After animals were euthanized, mice were transcardially perfused with $4^{\circ} \mathrm{C}$ PBS to remove blood both systemically (via left ventricle) and within the pulmonary vasculature (right ventricle). Lungs were placed into $4^{\circ} \mathrm{C}$ IMDM (Gibco) until tissue collection was completed. Lungs were then minced and incubated at $37^{\circ} \mathrm{C}$ for $40 \mathrm{~min}$, on a shaker, in petri dishes filled with $5 \mathrm{ml}$ IMDM containing penicillin, streptomycin, and $183 \mathrm{u} / \mathrm{ml}$ collagenase type II (Worthington). Digested lungs were gently pressed and filtered 
through a $70 \mu \mathrm{m}$ filter, washed in $50 \mathrm{ml}$ flow cytometry buffer, centrifuged at $4^{\circ} \mathrm{C}$ for 10min, 300g, decanted, RBC lysis was performed with ACK buffer (same method as described in Preparation of blood for flow cytometry), and cells were used for flow cytometry staining. Lung cell counts were obtained by acridine orange / propidium iodide staining solution in PBS and analysis on a Nexcelom Cellometer Auto 2000. Total lung cell counts were obtained by multiplication of the percentage of each population among total live cells measured via flow cytometry by the total live cell count.

\section{Flow cytometry of mouse samples}

After tissue preparation, cells were stained for $30 \mathrm{~min}$ at $4^{\circ} \mathrm{C}$ in flow cytometry buffer $(1 \%$ BSA, 2mm EDTA, and $0.1 \%$ sodium azide in PBS). All flow cytometry antibodies used were purchased from BD pharmingen or eBioscience, except for CD115 (Biolegend), and CCR2 (R\&D Systems). Tetramers were from MBL (OTI tetramer) or the Trudeau institute (NP and PA tetramers). For tetramer staining, samples were incubated for 30min at room temperature. Samples were washed with $5 \mathrm{ml}$ flow cytometry buffer, centrifuged for $10 \mathrm{~min}$ at $300 \mathrm{~g}, 4^{\circ} \mathrm{C}$, and decanted. For intracellular staining, samples that had already been stained with extracellular antibodies were fixed and permeabilized using IC fixation buffer (eBioscience) and stained/washed using permeabilization buffer (eBioscience), according to manufacturer instructions. Samples were either fixed in 1\% PFA for later analysis or immediately run on a flow cytometer. Cytometers used were an LSR Fortessa (BD) in the University of Virginia Flow Cytometry Core, and a Gallios (Beckman Coulter) in the Center for Brain Immunology and Glia, University of Virginia. 


\section{Transfer of OTI T cells and assessment of proliferation during infection}

$\mathrm{OTI}^{+/-} \mathrm{CD} 8+\mathrm{T}$ cells were sorted by positive selection from either wild type or MeCP2 $2^{\mathrm{Tg} 3}$ / $\mathrm{OTI}^{+/-}$donors using CD8+ selection magnetic beads (Miltenyi) per manufacturer instructions. Sorted $\mathrm{OTI}^{+/-} \mathrm{CD} 8+\mathrm{T}$ cells were CFSE labeled and 2 million cells were injected via tail vein into each host. 1 day later, mice were infected with OVA-expressing influenza $\mathrm{A} / \mathrm{PR} / 8$. On day 3.5 , mice were sacrificed and mediastinal lymph nodes were collected to assess for $\mathrm{OTI}^{+/-} \mathrm{CD} 8+\mathrm{T}$ cell proliferation by CFSE dilution.

\section{Culture of BMDC and re-stimulation of T cells with virus loaded BMDC}

Bone marrow was collected from femurs and resuspended in DC media (RPMI without L-glutamine, $10 \%$ FBS, Pen/Strep, $0.05 \mathrm{mM}$ beta mercaptoethanol, and $2 \mathrm{mM}$ Lglutamine) at a concentration of $10^{7}$ cells per $\mathrm{ml} .10 \mathrm{ml}$ of DC media containing $20 \mathrm{ng} / \mathrm{ml}$ GM-CSF (eBioscience) was placed in a non-TC treated $100 \mathrm{~mm}$ petri dish, and $200 \mu \mathrm{l}$ of the concentrated bone marrow $\left(2 \times 10^{6}\right.$ bone marrow cells $)$ were carefully pipetted as a colony in the middle of the petri dish. After resting for $10 \mathrm{~min}$ to allow cells to settle, petri dishes were moved to a tissue culture incubator at $37^{\circ} \mathrm{C}$, with care taken not to disperse the concentrated bone marrow cells in the center of the dish. On day 3, an additional $10 \mathrm{ml}$ of DC media containing 20ng/ml GM-CSF was slowly added to each petri dish so as not to disturb the cells. On days 6-9, BMDC were harvested by collecting floating and semi-adherent cells in each petri dish. Cells were washed with serum-free media (RPMI) twice. BMDC pellets were resuspended in $200 \mu \mathrm{l}$ serum-free media, and mixed with $500 \mu \mathrm{l}$ stock influenza $\mathrm{A} / \mathrm{PR} / 8 / 34$ virus for $5 \mathrm{~min}$ on ice, followed by incubation at $37^{\circ} \mathrm{C}$ 
for $30 \mathrm{~min}$. Then $20 \mathrm{ml}$ of pre-warmed $37^{\circ} \mathrm{C}$ DC media containing $20 \mathrm{ng} / \mathrm{ml}$ GM-CSF was added, and cells were incubated at $37^{\circ} \mathrm{C}$ for 5 hrs. BMDC were then washed with DC media, counted, and mixed with effector cells (prepared via lung digestion, as described) at a ratio of 1:1, in a 6 well non-TC treated plate. Cells were incubated at $37^{\circ} \mathrm{C}$ for $1 \mathrm{hr}$, and then Brefeldin A (eBioscience) was added to each well, followed by an additional $4 \mathrm{hr}$ incubation at $37^{\circ} \mathrm{C}$. Cells were then collected and stained for cytokine production by flow cytometry.

\section{Anti-PR8 antibody ELISA}

96-well ELISA plates were coated with influenza A/PR/8/34 virus stock diluted 1:100 in PBS $\left(7 \times 10^{7}\right.$ EID $_{50}$ per $\left.\mathrm{ml}\right)$ overnight at $4^{\circ} \mathrm{C}$. Plates were then washed three times with PBS containing $0.05 \%$ Tween-20 (PBST). Plates were then blocked with $2 \%$ BSA in PBST for $1 \mathrm{hr}$, room temperature, and washed three times with PBST. BAL fluid was then added to each well (50 $\mu$ l volume) starting at 1:10 BAL diluted in PBS and then serially diluted 1:5. Samples were incubated at room temperature for $2 \mathrm{hrs}$, and then wells were washed three times with PBST. Anti-mouse IgG or IgM conjugated to horseradish peroxidase (HRP) (SouthernBiotech) was diluted 1:10,000 in PBS and 50 $\mu$ l was added to each well for $1 \mathrm{hr}$ at room temperature. Wells were again washed three times with PBST, and TMB substrate was added to each well. $100 \mu 12 \mathrm{~N} \mathrm{H}_{2} \mathrm{SO}_{4}$ stop solution was added to each well to stop the enzymatic reaction, and OD was measured at $450 \mathrm{~nm}$ on a $96-w e l l$ plate reader. 


\section{TCID $_{50}$ assay}

Madin-Darby canine kidney cells (MDCK) were grown in MEM supplemented with 10\% FBS and 1\% Pen/Strep. Once cells reached $80 \%$ confluency, they were harvested by trypsinization $\left(5 \mathrm{~min}, 37^{\circ} \mathrm{C}\right)$ and washed twice with media to quench trypsin. MDCK were then counted, split 1:4, and cultured overnight. The next day, cells were again harvested as described above, replated into 96-well plates, and allowed to adhere overnight at $37^{\circ} \mathrm{C}$. The following day, serum was washed off the cells using serum-free media, twice. Positive control virus, negative control (PBS), or BAL fluid was prepared by mixing an initial $20 \mu \mathrm{l}$ of sample with $180 \mu \mathrm{l}$ of serum-free media containing trypsin, and serially diluting 1:10, 7 times. Serial dilutions were added to MDCK in 96-well plates, in triplicate, and incubated for 3 days. 96-well plates were spun down, and $50 \mu 1$ supernatant was pipetted into new 96 -well round bottom plates. $50 \mu 1$ of $1 \%$ chicken red blood cells in PBS were added and mixed. Plates were allowed to incubate at room temperature for $30 \mathrm{~min}$ and hemagglutination was assessed. $\mathrm{TCID}_{50}$ was reported as the average number of wells with hemagglutination between technical replicates for each sample.

\section{Complete blood count (CBC) in mice}

For serial complete blood counts (CBC), lateral tail veins were lanced every other day and a minimum amount of blood was taken for analysis $(\sim 50 \mu 1)$. Samples were collected into EDTA tubes for anti-coagulation and analyzed on a VetScan Abaxis HM5 CBC analyzer. 


\section{Bronchoalveolar lavage}

After mice were euthanized, skin and muscle over the trachea was removed, a small incision made in the trachea, a $25 \mathrm{G}$ needle sheathed with soft plastic tubing was inserted into the trachea, secured with sutures, and used to administer $0.5 \mathrm{ml}$ PBS to lavage the lungs. Supernatants from the first lavage were used for cytokine, viral titer, and antibody titer analysis. For flow cytometry analysis, an additional $1 \mathrm{ml}$ of PBS was used to lavage the lungs a second time, and cells from both lavages were used for flow cytometry staining.

\section{Luminex analysis of mouse BAL fluid}

BAL fluid samples were collected as described and stored at $-80^{\circ} \mathrm{C}$ until analysis. BAL fluid samples were submitted to and analyzed by the University of Virginia Flow Cytometry Core using Milliplex reagents per manufacturer's instructions (EMD Millipore).

\section{Corticosterone measurement}

Serum samples were collected after animal sacrifice via blood collection from the eye. Briefly, the eye was removed, and blood was collected in a BD microtainer serum gel tube. Blood was allowed to fully clot for $45 \mathrm{~min}$ at room temperature and serum was collected by centrifugation at $15,000 \mathrm{~g}$ for $90 \mathrm{sec}$ and collection of supernatant (serum). Serum samples were frozen at $-80^{\circ} \mathrm{C}$ until analysis by the University of Virginia Ligand Assay and Analysis Core using a mouse and rat corticosterone ELISA (IBL). 


\section{Celecoxib and mifepristone treatment}

Celecoxib (LKT laboratories) and/or mifepristone (Sigma) was suspended in $0.4 \%$ carboxymethylcellulose, low-viscosity (Sigma) in PBS. Suspensions were prepared by vortexing followed by brief sonications in a water bath sonicator. Doses used were 25 or $100 \mathrm{mg} / \mathrm{kg}$ for celecoxib, and $25 \mathrm{mg} / \mathrm{kg}$ mifepristone, given by daily intraperitoneal injection starting $1 \mathrm{hr}$ prior to infection.

\section{Bone marrow transplant}

4-5 week old mice were lethally irradiated with 1000 rad, and injected with 5 million bone marrow cells via tail vein 5 hours post irradiation. Hosts were C57BL6 x FVB F1 hybrids (either $\mathrm{MeCP} 2^{\mathrm{Tg} 3}$ or wild type), and donor bone marrow was of the identical background strain. Donor mice were either $\mathrm{MeCP} 2^{\mathrm{Tg} 3}$ or wild type, and all donors were

additionally $\mathrm{UBC}^{\mathrm{GFP} /+}$ in order to allow for assessment of engraftment efficiency prior to use of mice in downstream experiments. Mice were given water containing sulfamethoxazole and trimethoprim for two weeks post-transplant. Infection experiments were performed 10 weeks post-transplant after verification of robust GFP + peripheral immune cell engraftment (typically $>90 \%$ ).

\section{Evans blue assay for assessment of vascular permeability}

Mice were injected intraperitoneally with $400 \mu 1$ 2\% Evans Blue solution in PBS 1 hour prior to collection. Mice were euthanized by Euthasol, and transcardially perfused with ice cold PBS both systemically (left ventricle) and throughout the pulmonary vasculature 
(right ventricle) to remove vascular blood and Evans Blue dye. After weighing and photographing organs and BAL fluid, samples were mixed with one volume of $50 \%$ trichloroacetic acid and incubated overnight at $4^{\circ} \mathrm{C}$ to precipitate out proteins and other particulates. Samples were centrifuged at $15,000 \mathrm{~g}$ for $30 \mathrm{~min}, 4^{\circ} \mathrm{C}$. Supernatants were analyzed by 96-well plate reader at 620nm (Multiskan FC, Fisher Scientific) and a standard curve was generated using known concentrations of Evans Blue dye in PBS mixed 1:1 with 50\% trichloroacetic acid.

\section{Histology}

Tissues were collected either with or without vascular perfusion and inflation of lungs with $4 \%$ PFA as indicated in each figure. Tissues were placed in $4 \%$ paraformaldehyde (PFA) for 2 days, followed by transfer to $70 \%$ EtOH. Tissues were submitted to the University of Virginia Research Histology Core, where they were paraffin embedded, sectioned, mounted, and stained for hematoxylin and eosin (H\&E).

\section{Pulmonary function measurement}

We measured the lung function using a buffer-perfused mouse lung system (Hugo Sachs Elektronik). Briefly, mice were anesthetized with ketamine and xylazine and ventilated with room air at 100 strokes/min with a tidal volume of $7 \mu 1 / \mathrm{g}$ body weight with a positive end expiratory pressure of $2 \mathrm{~cm} \mathrm{H}_{2} \mathrm{O}$ using the MINIVENT mouse ventilator (Hugo Sachs Elektronik). The animals were exsanguinated by transecting the inferior vena cava. The pulmonary artery was cannulated via the right ventricle, and the left 
ventricle was immediately tube-vented through a small incision at the apex of the heart. The lungs were then perfused at a constant flow of $60 \mu 1 / \mathrm{g}$ body weight/min with KrebsHenseleit buffer containing $2 \%$ albumin, $0.1 \%$ glucose, and $0.3 \%$ HEPES. The perfusate buffer and isolated lungs were maintained at $37^{\circ} \mathrm{C}$ throughout the experiment. Isolated lungs were allowed to equilibrate on the apparatus during a 5-min stabilization period. After equilibration, data were recorded for an additional 10 minutes. Hemodynamic and pulmonary parameters were continuously recorded during this period by the PULMODYN data acquisition system (Hugo Sachs Elektronik).

\section{Statistics and analysis of data}

Flow cytometry data was analyzed using FlowJo (TreeStar). All statistics were performed in Prism 5 or 6 (GraphPad) using the statistical tests described in each figure legend. 


\section{Chapter 3}

\section{MeCP2 regulates transcriptional response to stimuli in macrophages}

*NOTE: The original publication and figures discussed in this chapter can be found in the Appendix. Figures appearing in the original publication are referred to with an "A" before the figure number, e.g. Fig. A1, to denote their appearance in the appendix as opposed to the main chapters.*

We had previously shown that wild type bone marrow transplant into Mecp2-null mice mediates significant improvement in both phenotype and survival via the CNS engraftment of new microglia-like macrophages (Derecki et al., 2012). This was demonstrated by comparing survival of mice that had their heads covered with a lead shield during irradiation, or not, prior to bone marrow transplant. When the head is covered with a lead shield during lethal irradiation, no engraftment of peripheral-derived 
macrophages occurs in the CNS, as we confirmed in our original study (Derecki et al., 2012). When Mecp2-null mice were protected from head irradiation, and thus CNS engraftment of peripheral macrophages was prevented, survival benefit from bone marrow transplant was abolished. Based on this, we concluded that engraftment of wild type peripheral-derived macrophages into the CNS was essential for benefits in Mecp2null mice. At the time, we called these engrafting cells "microglia," however today we know this term is incorrect, as peripheral-derived engrafting CNS macrophages are unique from microglia (Butovsky et al., 2014; Waisman et al., 2015). Likewise, we demonstrated similar survival and phenotypic improvements in Lysm ${ }^{\text {Cre }} /$ Mecp $2^{\text {LoxStop.y }}$ mice, which re-express Mecp2 in some microglia and peripheral myeloid cells.

While we demonstrated that engraftment of macrophages into the CNS after bone marrow transplant into Mecp2-null mice was critical for survival and phenotypic improvement (Derecki et al., 2012), we did not explore the potential contribution of peripheral immunity to disease pathogenesis in Mecp2-null mice. Further, in the context of both bone marrow transplant and $\mathrm{Lysm}^{\mathrm{Cre}} / \mathrm{Mecp} 2^{\text {LoxStop/y }}$ mice, peripheral myeloid cells were genetically rescued for Mecp2 expression, raising the possibility that peripheral myeloid cells play a role in disease and require Mecp2 for their proper function. Further, we found in our own experiments that contrary to previous claims (Chen et al., 2001; Guy et al., 2001), Nestin ${ }^{\text {Cre }} / \operatorname{Mecp} 2^{\text {Flox/y }}$ mice, while sick and demonstrating reduced lifespan, had significantly increased lifespan above complete Mecp2-null mice (Fig. 1). This indicated to us that neurons and non-immune glia do not account for the full extent of disease in Mecp2-null mice, leaving open the possibility that 
other cells, such as microglia and macrophages, may contribute to disease pathogenesis. We therefore endeavored to answer two questions; 1) do peripheral macrophages, in addition to microglia, express Mecp2, and 2) what is the functional role of Mecp2 in microglia and macrophages?

In our publication entitled "Methyl-CpG binding protein 2 regulates microglia and macrophage gene expression in response to inflammatory stimuli" we demonstrated that not only do microglia require Mecp2 for their proper health and function, but that macrophages throughout the body express Mecp2, and that Mecp2 regulates transcriptional responses to stimuli in macrophages, including glucocorticoid, hypoxic, and inflammatory signaling (Cronk et al., 2015a). The full publication can be found in the Appendix, and the results of this study are summarized below.

In Fig. A1 and Fig. AS1 in the original publication, we demonstrated that microglia express Mecp2, and that Mecp2-null mice lose microglia with disease progression. This is in agreement with a contemporaneous publication, which also showed loss of microglia in Mecp2-null mice (Jin et al., 2015). In unpublished experiments, we also confirmed that Mecp2 was expressed in a chimeric fashion by microglia in Mecp2-heterozygous female mice, as expected (Fig. 2). In addition, wild type mice always expressed Mecp2 in nearly all microglia, while Mecp2-null mice always demonstrated clear lack of Mecp2 expression in microglia.

In Fig. A1, we also demonstrated that microglia from late-phenotypic Mecp2-null mice have reduced process complexity and increased soma size, consistent with an inflammatory "activated" microglial state. This was corroborated by qPCR measurement 
of both increased tumor necrosis factor (Tnf) expression and decreased transforming growth factor beta 1 (Tgfbl) expression, which indicated that Mecp2-null microglia become activated in the late-phenotypic disease state. Finally, we found that while prephenotypic Mecp2-null mice had normal microglia numbers, late-phenotypic Mecp2-null mice developed a pronounced deficit of microglia throughout the brain.

In Fig. A2 we continued our investigation of myeloid cells in the context of Mecp2 deletion by examining the most proximal population of macrophages to microglia: meningeal macrophages. Like microglia, perivascular meningeal macrophages were lost with disease progression, suggesting that Mecp2 may be important to macrophages outside of the CNS parenchyma. However, we found that non-perivascular meningeal macrophages were not significantly depleted, potentially indicating population-specific effects of Mecp2 among macrophage populations.

Since we had observed loss of both CNS macrophages (microglia) and a peripheral macrophage population (perivascular meningeal macrophages), we investigated the status of other peripheral macrophage populations (Fig. A3). Here, we demonstrated that every peripheral macrophage population examined (including bone marrow resident macrophages, splenic red pulp macrophages, peritoneal resident macrophages, and intestinal lamina propria macrophages) and resident Ly6 $\mathrm{C}^{\text {Lo }}$ monocytes all express Mecp2. Interestingly, we found that neither neutrophils nor Ly6 $\mathrm{C}^{\mathrm{Hi}}$ monocytes expressed detectable Mecp2 as measured by flow cytometry, which we interpreted to indicate that Mecp2 might be preferentially expressed by longer-lived, differentiated monocytes/macrophages, as opposed to short-lived inflammatory myeloid 
cells. We continued on to demonstrate that like microglia and perivascular meningeal macrophages, both intestinal lamina propria macrophages and resident $\mathrm{Ly}_{6} \mathrm{C}^{\mathrm{Lo}}$ monocytes are also lost in Mecp2-null mice. Together, these cumulative pieces of evidence suggested that in the absence of Mecp2, multiple macrophage/monocyte populations are lost. We initially hypothesized that Mecp2-null macrophages may be impaired in their ability to proliferate, however analysis of bone marrow-derived macrophage (BMDM) proliferation from bone marrow monocytes in vitro revealed no such deficit (Fig. AS2). Some explanation for microglia and macrophage loss may be found in the work of others that was previously discussed in Chapter 1, in which it was demonstrated that abnormal glutamine metabolism and subsequent mitochondrial pathology, including increased ROS production, may account for microglia loss in Mecp2-null mice (Jin et al., 2015). It would be logical that a similar mechanism may affect other macrophages in the absence of Mecp2.

Based on our observations that Mecp2-null mice were deficient in monocytes and macrophages, we attempted to correct this deficit in unpublished experiments by either treatment with macrophage-colony stimulating factor (Mcsf), the major macrophage growth and survival factor (Fig. 3), or weekly injection of wild type monocytes into Mecp2-null mice (Fig. 4). However, neither treatment resulted in significant survival benefits in Mecp2-null mice. We hypothesized that Mcsf treatment may have been insufficient because even if we rescued macrophage numbers in Mecp2-null mice, those cells would still be functionally impaired due to lack of Mecp2. Similarly, since we had previously demonstrated that engraftment of peripheral macrophages into the CNS of 
Mecp2-null mice was critical for benefit (Derecki et al., 2012), we hypothesized that infusion of wild type monocytes into the periphery alone may be insufficient to mediate benefits without concurrent rescue of microglia. We thus decided to pursue genetic rescue of both microglia and macrophages, since this would avoid problems inherent to both of these therapeutic paradigms.

In our previous work, no tool to specifically target microglia and macrophages on the genetic level existed (Derecki et al., 2012). The Lysm $^{\text {Cre }}$ model we had previously used does target some microglia and peripheral monocytes/macrophages, but is also expressed significantly in other myeloid cell populations, such as neutrophils (Clausen et al., 1999). This model was therefore insufficient to claim for either microglia, or even macrophage, mediated rescue alone. Since then, a new mouse model became available: the $C \times 3 \mathrm{crl}^{\mathrm{CreER}}$ mouse. When treated with tamoxifen, this mouse demonstrates cre activity in nearly all microglia, and selected peripheral macrophage and dendritic cell populations (Goldmann et al., 2013; Yona et al., 2013). In order to more specifically measure the effect of genetic correction of microglia, and some peripheral macrophages, in the context of global Mecp2 deficiency, we crossed $C x 3 \mathrm{cr} 1^{\text {CreER }}$ mice to Mecp $2^{\text {LoxStop/y }}$ mice, as presented in Fig. A4. Here, we showed that genetic re-expression of Mecp2 in microglia and peripheral macrophages, including intestinal macrophages, mediated significant lifespan extension in otherwise Mecp2-null mice, supporting our previous assertions that genetic rescue of microglia (and based on this new evidence, potentially other macrophages) mediates significant benefits in otherwise Mecp2-null mice. 
Although we had measured expression of Mecp2 throughout macrophage populations, and loss of macrophages in Mecp2-null mice, the functional role of Mecp2 in macrophages and microglia remained an outstanding question. To address this, we performed RNA-Seq on microglia and peritoneal macrophages derived from latephenotypic Mecp2-null mice and their wild type counterparts (Fig. A5). Analysis of RNA-Seq profiles from microglia and peritoneal macrophages revealed common perturbed pathways, which included glucocorticoid signaling and hypoxia signaling, which were both confirmed to be dysregulated in Mecp2-null macrophages in vitro using wild type and Mecp2-null BMDM. We found that Hdac3 and Ncor2 were bound to the promoter of FK506 binding protein 5 (Fkbp5), a key glucocorticoid response gene (Fig. AS3). Importantly, Ncor is an important Mecp2 binding partner, as was previously discussed (Kokura et al., 2001; Lyst et al., 2013). In Fig. A5 we also found that Mecp2 was bound to the Fkbp5 promoter. Together, these results suggested that Mecp2 is involved in transcriptional regulation of signaling pathways in macrophages.

Our RNA-Seq analysis also indicated that Mecp2 may regulate inflammatory signaling; specifically, Tnf pathways. In support of this, we had already measured inflammatory activation of microglia from late-phenotypic Mecp2-null mice in Fig. A1. Therefore, in Fig. A6, we tested inflammatory response of Mecp2-null BMDM to Tnf stimulation. As expected, we found that Mecp2-null macrophages were hyper-responsive to Tnf stimulation, overproducing inflammatory cytokines, chemokines, and growth factors. We also stimulated resident peritoneal macrophages with Tnf in vivo, and found dysregulation of Tnf response genes in this paradigm as well. Importantly, this was in 
direct response to Tnf, and not baseline outside inflammatory stimulation due to genetic disease, as saline treated Mecp2-null mice showed no significant changes in Tnf-induced transcripts (Fig. AS4).

In unpublished results, we also found that lipopolysaccharide (LPS) substantially increased expression of Mecp2 by BMDM approximately 3-4 fold (Fig. 5). Interestingly, 6 hours of incubation with IFN $\gamma$ prior to LPS treatment attenuated the early (6hrs post LPS) increase in Mecp 2 expression, although by $18 \mathrm{hrs}$ post LPS Mecp 2 transcript levels had equalized with BMDM exposed to LPS alone (Fig. 5). These findings implicated that Mecp2 itself is dynamically regulated upon inflammatory stimulation, and that combinatorial signaling by additional cytokines, such as IFN $\gamma$, may confer temporal regulation of Mecp2 expression during an inflammatory process.

In both in vitro and in vivo experiments, the gene encoding granulocyte-colony stimulating factor (Csf3) was overexpressed upon Tnf stimulation (Fig. A6). This correlated with a previous unexplained finding in our immunologic analysis of Mecp2null mice; Mecp2-null mice develop a profound neutrophilia with disease progression (Fig. AS5). We hypothesized that overexpression of granulocyte-colony stimulating factor (Gcsf) protein in vivo may occur due to loss of Mecp2. Indeed, in Fig. A6 we found that phenotypic Mecp2-null mice had significantly elevated serum Gcsf levels. Moreover, when we neutralized Gcsf with a Gcsf neutralizing antibody, not only were neutrophil levels normalized, confirming Gcsf as the cause of neutrophilia in Mecp2-null mice, but also a moderate lifespan extension of approximately two weeks was observed. 
One finding that may help explain some of the benefit observed in our previous bone marrow transplant experiments in Mecp2-null mice (Derecki et al., 2012) may lie in the fact that Mecp2-null mice progressively lose hematopoietic stem cells (HSC) with disease progression, as was demonstrated in Fig. AS5. It is possible that in addition to new macrophage engraftment into the CNS (and in light of the findings discussed here, replacement of peripheral macrophages), rescue of the hematopoietic niche with a new infusion of wild type HSC may have also accounted for an important aspect of disease rescue in the context of wild type bone marrow transplant into Mecp2-null mice.

Overall, the findings presented in this work demonstrated that 1) Mecp2 is broadly expressed in macrophages and resident monocytes; 2) re-expression of Mecp2 in only microglia and select peripheral macrophages results in significant lifespan extension; and 3) Mecp2 regulates glucocorticoid, hypoxia, and inflammatory signaling pathways in macrophages. These findings significantly advanced our understanding of the role of Mecp2 in microglia and macrophages, and more globally, the potential role of these cells in disease. 


\section{Figures}

Figure 1.

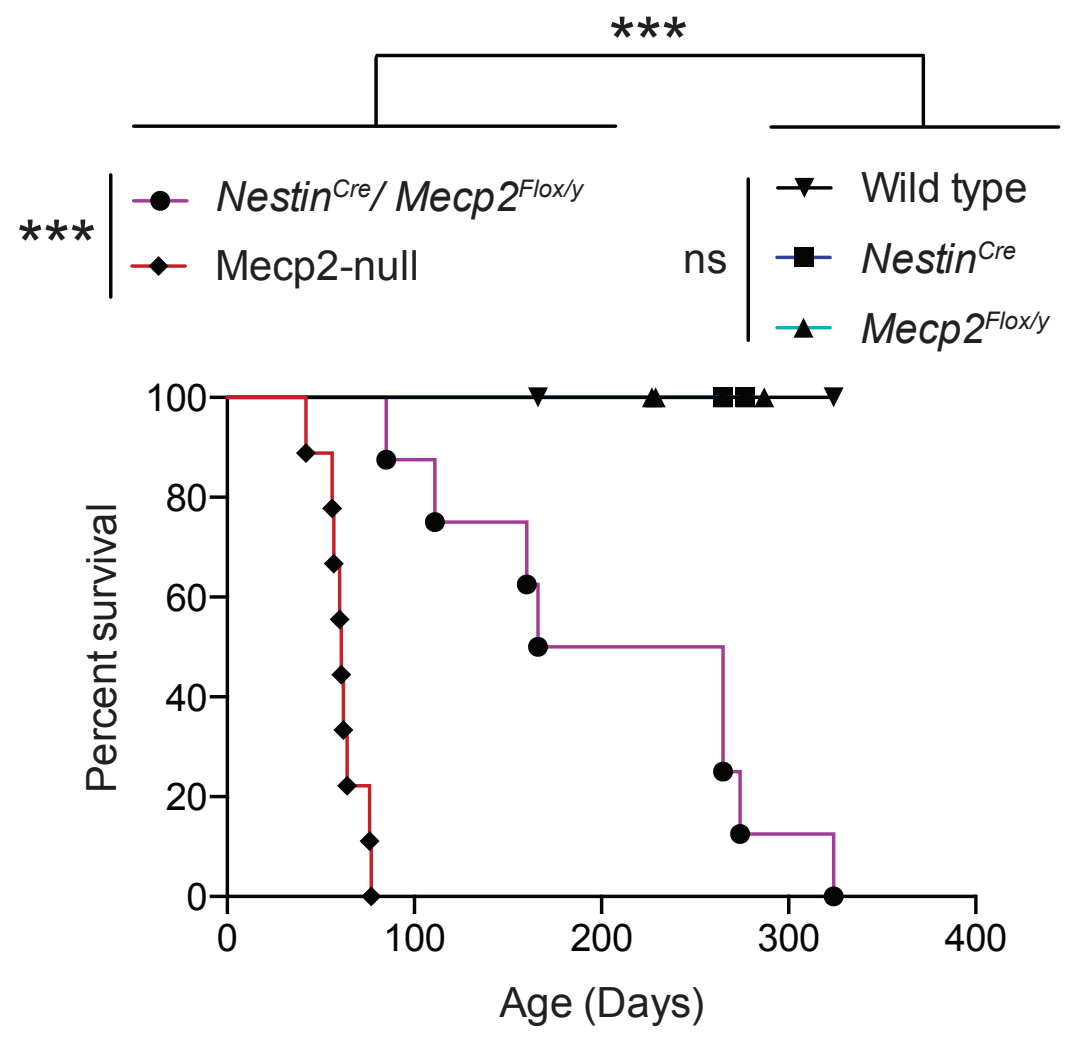

Nestin $^{\text {Cre }}$ mediated deletion of Mecp2 does not fully recapitulate the reduced survival observed in Mecp2-null mice. Mecp $2^{\text {Flox }}$ mice were crossed to Nestin ${ }^{\text {Cre }}$ mice and survival was compared to wild type, Mecp2-null, Nestin ${ }^{\text {Cre }}$, and Mecp $2^{\text {Flox/y }}$. Log-rank (Mantel-Cox) test, ***, $\mathrm{p}<0.001 . \mathrm{n}=8$ Nestin $^{\text {Cre }} /$ Mecp $^{\text {Flox/y }}, \mathrm{n}=9$ Mecp2-null, $\mathrm{n}=5$ Nestin ${ }^{\text {Cre }}, \mathrm{n}=8 \operatorname{Mecp} 2^{\text {Flox/y, }}$ and $\mathrm{n}=4$ wild type. 
Figure 2.
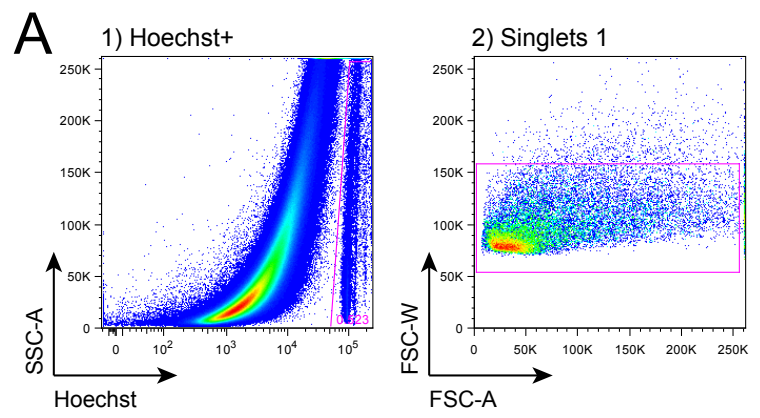

B
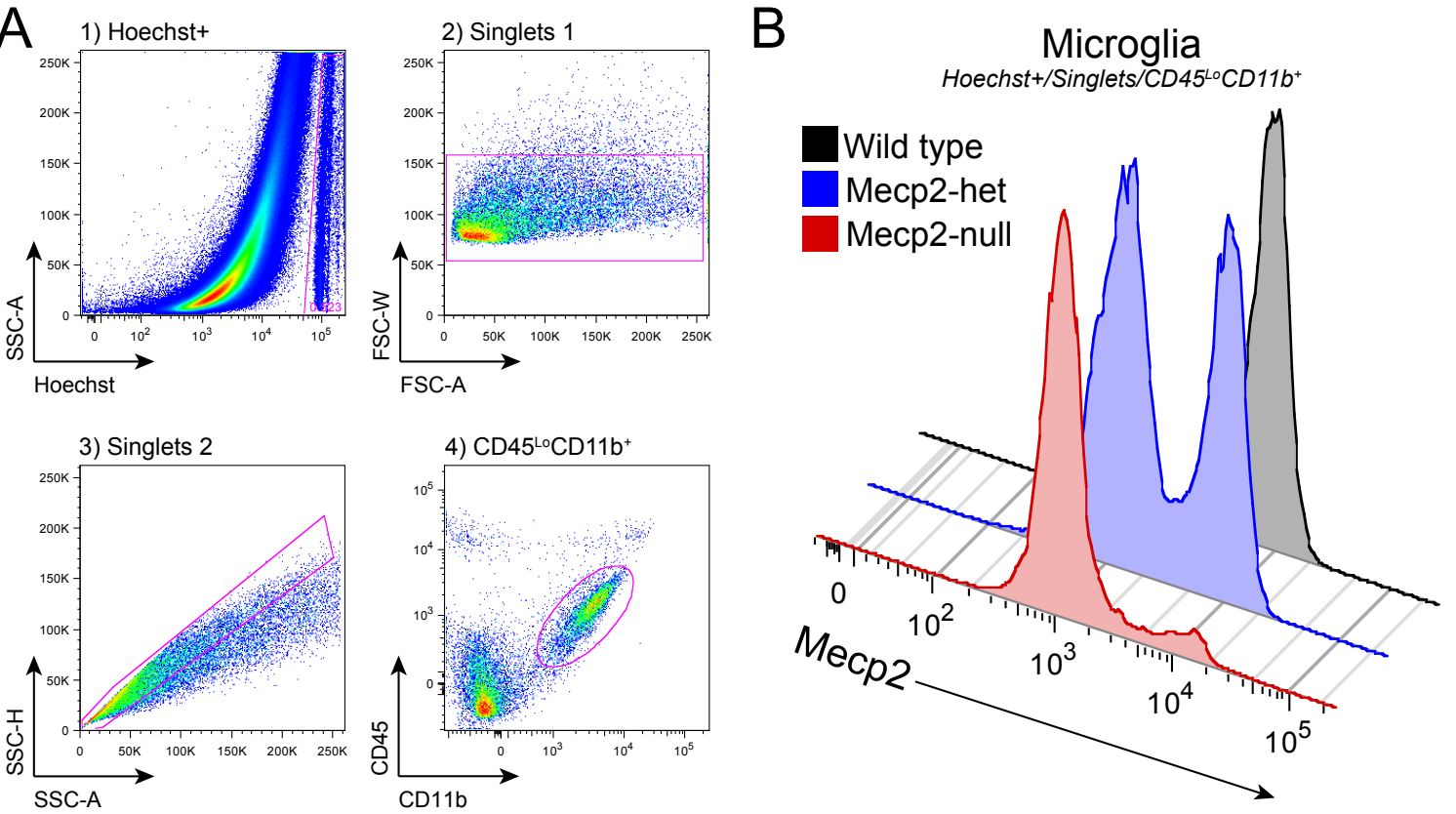

Flow cytometric staining for Mecp2 in microglia of Mecp2-heterozygous female mice reveals chimeric expression of Mecp2 in microglia. (A) Example gating strategy for microglia. (B) Intracellular flow cytometry staining of microglia from wild type female, Mecp2-heterozygous female, and Mecp2-null male mice demonstrating Mecp2 expression in all wild type microglia, lack of Mecp2 expression in microglia from Mecp2-null mice, and chimeric expression of Mecp2 in microglia in Mecp2heterozygous females, as expected for all groups. Results are representative of at least three independent experiments with at least 3 mice per group for all groups. 
Figure 3.

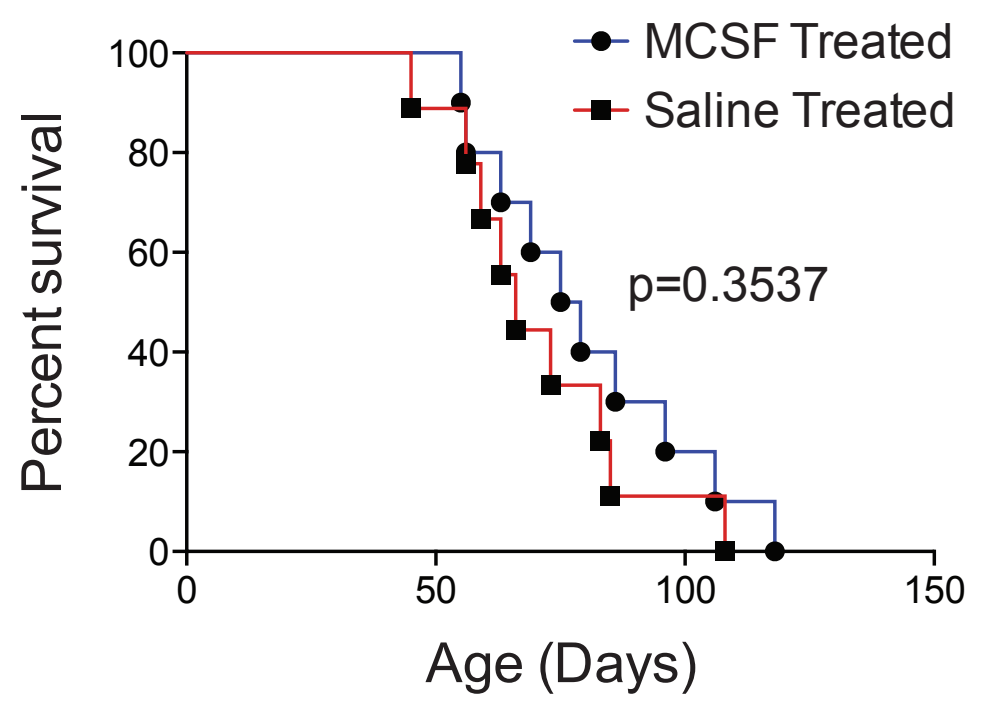

Mcsf treatment does not improve survival in Mecp2-null mice. Mecp2-null mice were injected weekly with either saline or $100 \mu \mathrm{g} / \mathrm{kg}$ Mcsf and survival was assessed. Log-rank (Mantel-Cox) test, not significant. $\mathrm{n}=10 \mathrm{Mcsf}$ treated and $\mathrm{n}=9$ saline treated Mecp2-null mice. 


\section{Figure 4.}

A

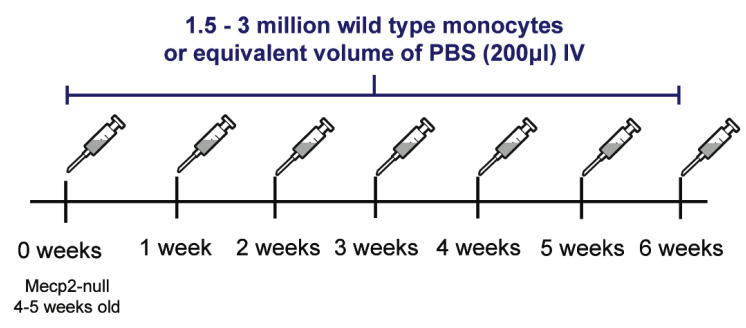

B

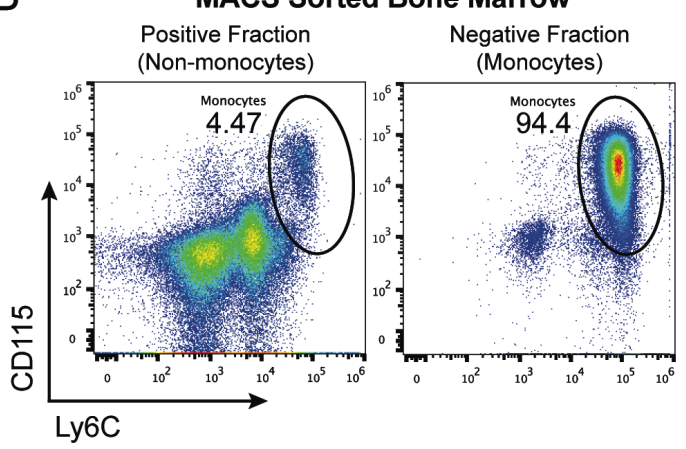

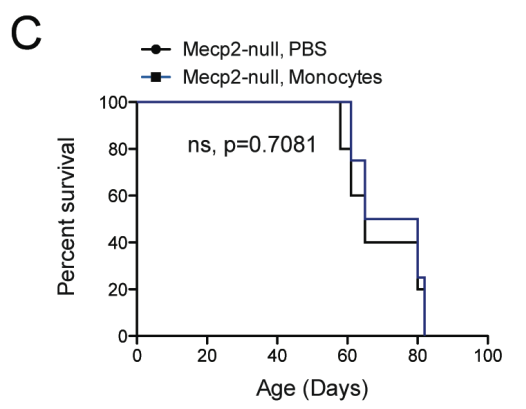
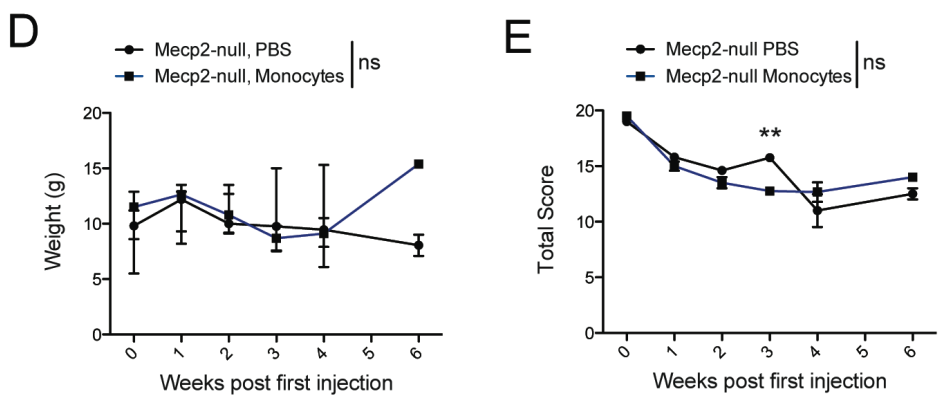

F
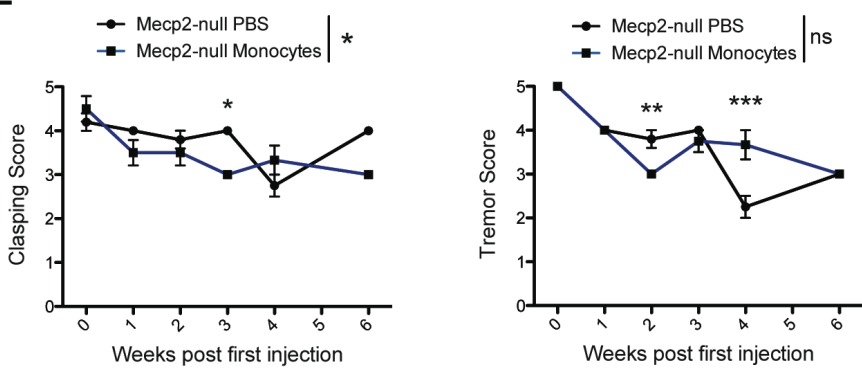

$\rightarrow$ Mecp2-null PBS
$\rightarrow$ Mecp2-null Monocytes
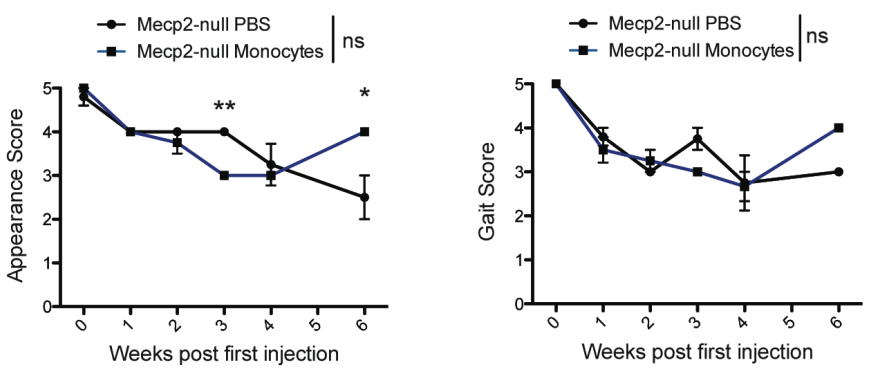
Wild type monocyte transfer does not improve survival in Mecp2-null mice. Total bone marrow monocytes from wild type mice were MACS sorted using the Miltenyi mouse bone marrow monocyte kit and transferred 1:1 into Mecp2-null hosts. Average total monocytes injected into each mouse was 1.5-3 million per week. (A) Schematic of the experiment; mice received up to 7 injections of monocytes or PBS. (B) Example flow cyotmetry plots of monocyte MACS sort demonstrating $90 \%+$ pure monocytes. (C) Survival curve demonstrating no significant effect, Log-rank (Mantel-Cox) test; $\mathrm{n}=4$ monocyte injected and n=5 PBS injected Mecp2-null. (D) No significant difference in weight. (E) No overall difference in total phenotypic score except at 3 weeks; two-way ANOVA with Bonferroni post test, ${ }^{* *}, \mathrm{p}<0.01$. (F) Individual phenotype scores. 5= wild type phenotype, $1=$ moribund/end stage phenotype with numbers in between representing progression of disease; two-way ANOVA with Bonferroni post test, *, p $<0.05$; **, $\mathrm{p}<0.01 ; * * *, \mathrm{p}<0.001$. 
Figure 5.

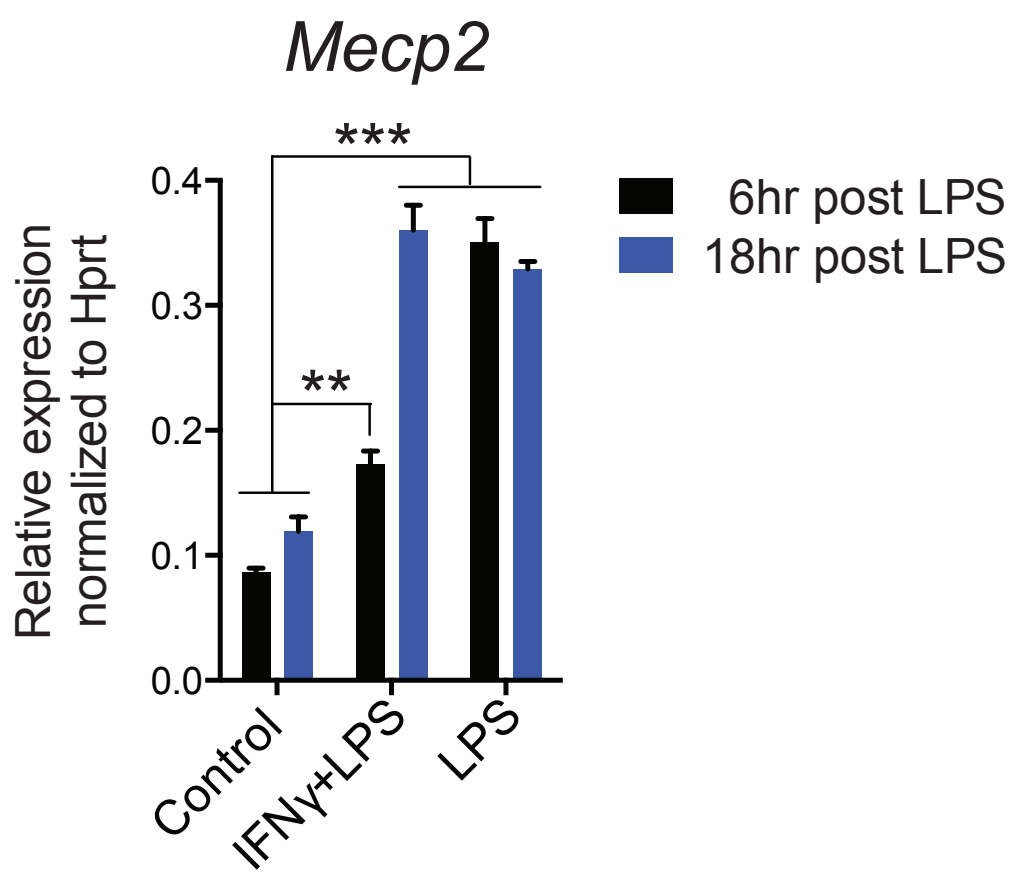

LPS induces Mecp2 expression in BMDM, which is attenuated by priming with IFN $\gamma$. Wild type BMDM were either primed with $50 \mathrm{ng} / \mathrm{ml}$ IFN $\gamma$ or control media for 6 hours, followed by stimulation with $50 \mathrm{ng} / \mathrm{ml}$ LPS for either 6 or $18 \mathrm{hrs}$. At the conclusion of the experiment, RNA was collected and qRT-PCR performed for Mecp2 transcripts. 


\section{Chapter 4}

\section{Lethal outcome and dysfunctional immune response to influenza $A$ in an animal model of MeCP2 overexpression}

\section{Background}

MeCP2 duplication syndrome is in part characterized by chronic respiratory infections, which cause significant morbidity and mortality in these patients (Friez et al., 2006; Ramocki et al., 2010). It was previously shown by ourselves and others that MeCP2 plays an important role in immune cells, including T cells (Balmer et al., 2002; Jiang et al., 2014; Li et al., 2014; Yang et al., 2012) and myeloid cells (Cronk et al., 2015a; Derecki et al., 2012; Jin et al., 2015; Maezawa and Jin, 2010; O'Driscoll et al., 2013b; O'Driscoll et al., 2015), and thus it was hypothesized that chronic infections seen in MeCP2 
duplication syndrome may be explained by the direct role of $\mathrm{MeCP} 2$ in immune cells. In 2012, Yang et al. demonstrated that CD4+ T cells overexpressing MeCP2 in both mice and humans were deficient in IFN $\gamma$ production after skewing to Th1 in vitro, and that $\mathrm{MeCP}^{\mathrm{Tg} 3}$ mice (Collins et al., 2004), which have a human MeCP2 gene inserted into their genome and overexpress $\mathrm{MeCP} 2$ at 3-5 fold normal levels, also demonstrate impaired IFN $\gamma$ levels during infection in vivo (Yang et al., 2012). This defect in IFN $\gamma$ production was hypothesized to account for the chronic infections seen in MeCP2 duplication syndrome (Yang et al., 2012). Importantly, Yang et al. demonstrated that transfer of wild type $\mathrm{CD} 4+\mathrm{T}$ cells into $\mathrm{MeCP} 2^{\mathrm{Tg} 3}$ mice decreased footpad swelling and increased IFN $\gamma$ levels during Leishmania major infection (Yang et al., 2012), suggesting that $\mathrm{CD} 4+\mathrm{T}$ cells expressing normal levels of $\mathrm{MeCP} 2$ have the potential to ameliorate immune pathology in an MeCP2 overexpressing host.

More recently, defects in antibody production have been implicated in MeCP2 overexpression. In mice, it was shown that $\mathrm{MeCP} 2$ overexpression leads to development of anti-nuclear antibodies (Koelsch et al., 2013). In patients, deficiency of $\operatorname{IgA}$ and $\operatorname{IgG}_{2}$ and excessive levels of $\mathrm{IgG}_{1}$ and $\mathrm{IgG}_{3}$ were found, in addition to an elevated acute phase response (Bauer et al., 2015). Interestingly, this same study of MeCP2 duplication patients did not detect any obvious $\mathrm{T}$ cell abnormalities, including normal IFN $\gamma$ production (Bauer et al., 2015).

Here, we demonstrate that $\mathrm{MeCP} 2^{\mathrm{Tg} 3}$ mice are significantly more susceptible to influenza A infection compared to their wild type counterparts. All $\mathrm{MeCP} 2^{\mathrm{Tg} 3}$ mice succumbed between days 6-9 post-infection at a viral dose where the majority of wild 
type mice survive. We further demonstrate that although $\mathrm{MeCP} 2^{\mathrm{Tg} 3}$ mice had an uneventful early response to infection, by day 5 a host of detrimental processes began to be manifested, including cytokine storm, peripheral and pulmonary neutrophilia, and excessive corticosterone levels. In mice that survived to days 7-8, defective adaptive immune responses were observed with failure of virus clearance and excessive virus accumulation in the lungs. Pharmacologic targeting of neither cytokine storm nor corticosterone was effective in preventing mortality, although some improvement in weight loss was observed. Despite the prevalence of immune defects, bone marrow transplant revealed that the $\mathrm{MeCP} 2^{\mathrm{Tg} 3}$ hematopoietic system was insufficient to explain mortality. Further investigation revealed that $\mathrm{MeCP} 2^{\mathrm{Tg} 3}$ mice experience significant vascular pathology during infection, including pulmonary hemorrhage, reduced accumulation of fluid/proteins/cells into the airways by days $7-8$, and high pulmonary artery pressure with histologic evidence of pulmonary arterial edema and luminal narrowing. Together, these results suggest that susceptibility to influenza A infection in the context of MeCP2 overexpression involves pathologic responses of both immune cells and non-hematopoietic cells, and that non-hematopoietic cells play a key role in infection-related mortality. 
Chapter 4: Lethal outcome and dysfunctional immune response to influenza $A$

in an animal model of MeCP2 overexpression

\section{Results}

\section{$\mathrm{MeCP2}^{\mathrm{Tg} 3}$ mice are highly susceptible to influenza $\mathrm{A}$ infection, with a normal initial} immune response followed by adaptive immune failure. We chose to breed $\mathrm{MeCP} 2^{\mathrm{Tg} 3}$ mice, which are commercially available on the FVB background, on a first generation C57BL6/J x FVB F1 cross to facilitate the use of genetic tools and reagents available for C57BL6 mice. Importantly, MeCP2 $2^{\mathrm{Tg} 3}$ mice only began to die of natural causes after 200 days, with over $50 \%$ of mice surviving to one year (Fig. 6A). No defects were observed in the baseline immune status of $\mathrm{MeCP} 2^{\mathrm{Tg} 3}$ mice, as measured by flow cytometry of peripheral blood (Fig. 6B-D). Because $\mathrm{MeCP} 2^{\mathrm{Tg} 3}$ mice did not begin to die of natural causes until approximately 28 weeks of age and appeared healthy for the first several months of life, we performed our experiments in adult $\mathrm{MeCP} 2^{\mathrm{Tg} 3}$ and wild type littermates between the ages of 10-15 weeks, with most experiments performed at 12 weeks of age.

It was previously demonstrated that $\mathrm{MeCP} 2$ overexpressing mice $\left(\mathrm{MeCP} 2^{\mathrm{Tg} 3}\right)$ and humans exhibit impaired IFN $\gamma$ production by Th1 CD4+ T cells, and this was suggested to be the primary etiology of chronic infection in $\mathrm{MeCP} 2$ duplication syndrome patients (Yang et al., 2012). However, it has also been shown that IFN $\gamma$-null mice are able to effectively respond to, and clear, primary influenza A infection (Graham et al., 1993). Thus, we hypothesized that if IFN $\gamma$ accounts for the major immune deficit in $\mathrm{MeCP} 2^{\mathrm{Tg} 3}$ mice, they should effectively clear primary influenza A infection. Surprisingly, $\mathrm{MeCP} 2^{\mathrm{Tg} 3}$ mice infected at 12 weeks of age universally succumbed to infection approximately 6-9 days post-infection (p.i.), demonstrating significantly increased 
mortality as compared to wild type mice infected with the same dose of virus (Fig. 7A). The percentage of body weight loss was similar between $\mathrm{MeCP} 2^{\mathrm{Tg} 3}$ and wild type mice (Fig. 7B).

Analysis of the host response in the lungs on day 3 p.i. revealed that immune cell counts were overall comparable between $\mathrm{MeCP} 2^{\mathrm{Tg} 3}$ and wild type mice (Fig. 7C). This is a time when the innate immune cells have entered the lungs in response to infection but prior to the onset of the adaptive immune response in the lungs, i.e. when the adaptive response is being initiated in the lung draining lymph nodes. In order to assess the ability of $\mathrm{MeCP} 2^{\mathrm{Tg} 3} \mathrm{~T}$ cells to mount an antigen-specific $\mathrm{CD} 8+\mathrm{T}$ cell response, we crossed $\mathrm{MeCP} 2^{\mathrm{Tg} 3}$ mice to TCR transgenic OTI mice to produce $\mathrm{MeCP}^{\mathrm{Tg} 3}:: \mathrm{OTI}^{+/-}$and wild type :: $\mathrm{OTI}^{+-}$littermates. OTI mice express, on CD8+ T cells, a transgenic TCR specific for chicken ovalbumin (OVA) (Hogquist et al., 1994), and thus these T cells can be used to assess antigen-specific CD8+ $\mathrm{T}$ cell responses to the OVA antigen. In order to assess the antigen-specific CD8 $+\mathrm{T}$ cell response in the context of influenza infection, 2 million wild type or $\mathrm{MeCP} 2^{\mathrm{Tg} 3}:: \mathrm{OTI}^{+/-} \mathrm{T}$ cells were transferred into wild type or $\mathrm{MeCP} 2^{\mathrm{Tg} 3}$ hosts. Twenty-four hours following $\mathrm{T}$ cell transfer, mice were infected with Influenza $\mathrm{A} / \mathrm{PR} / 8$ virus engineered to express the OVA epitope recognized by OTI T cells, and the antigen-specific $\mathrm{T}$ cell response in the draining mediastinal lymph node was assessed on day 3.5 p.i. We found that there was no difference in $\mathrm{T}$ cell proliferation based on genotype of the $\mathrm{T}$ cells, however $\mathrm{MeCP} 2^{\mathrm{Tg} 3}$ hosts actually had an increased number of OTI T cells and increased proliferation of OTI T cells on day 3.5 (Fig. 8A-C), suggesting 
that host genotype, rather than $\mathrm{T}$ cell genotype, may be driving any differences in influenza A response.

Although we found increased antigen-specific OTI $\mathrm{T}$ cell proliferation in $\mathrm{MeCP} 2^{\operatorname{Tg} 3}$ hosts on day 3.5 , we were surprised to find that the entire adaptive immune cell and antibody compliment in the lungs appeared to be blunted by day 8 p.i. T and B cell numbers in the $\mathrm{MeCP} 2^{\mathrm{Tg} 3}$ lung parenchyma were reduced, while innate immune cell numbers were normal or elevated at day 8 (Fig. 7D, Fig. 8D). Influenza A-specific antibody titers were greatly reduced in bronchoalveolar lavage (BAL) fluid (Fig. 7E and F), and influenza A virus titers were significantly elevated above wild type, in which no virus was detectable on day 8 (Fig. 7G). In addition to the reduced overall number of T cells, there was also a decreased percentage of influenza A-specific CD8+ T cells among total CD8+ T cells as measured by NP and PA tetramer staining (Fig. 8E). In agreement with this finding and previously published data (Yang et al., 2012), we found that both $\mathrm{CD} 4+$ and $\mathrm{CD} 8+\mathrm{MeCP} 2^{\mathrm{Tg} 3} \mathrm{~T}$ cells exhibited significantly reduced IFN $\gamma$ production upon restimulation by influenza A virus loaded bone marrow-derived dendritic cells (BMDC), with minimal differences based on the genotype of BMDC used as antigen presenting cells (APC), i.e. wild type or $\mathrm{MeCP} 2^{\mathrm{Tg} 3}$ bone marrow origin (Fig. 8F and G). Therefore, there was both a reduced percentage of IFN $\gamma$ producing $\mathrm{T}$ cells and a reduced overall $\mathrm{T}$ cell numbers, which likely combine to explain IFN $\gamma$ deficiency observed in the context of $\mathrm{MeCP} 2$ overexpression. Although this finding is consistent with previous published data (Yang et al., 2012), IFN $\gamma$-null mice have been shown to clear and survive primary 
Chapter 4: Lethal outcome and dysfunctional immune response to influenza A in an animal model of MeCP2 overexpression

influenza A virus infection (Graham et al., 1993), therefore it is unlikely that this explained the death of these mice in the context of influenza A infection.

\section{Influenza $\mathrm{A}$ infection of $\mathrm{MeCP}^{\mathrm{Tg} 3}$ mice results in severe lung pathology by five days}

post infection. Because the innate immune response appeared normal in these mice at day 3 p.i., but the adaptive response in the lungs was defective at day 8 , we decided to perform serial complete blood count $(\mathrm{CBC})$ measurements on peripheral blood to detect any obvious global changes occurring during the course of infection. These data revealed a robust and specific increase in granulocytes on day 5 p.i. (Fig. 9A). Interestingly, those mice that succumbed prior to blood collection on day 7 had a significantly elevated granulocyte count at day 5 p.i., while those that survived to day 7 did not have significantly elevated granulocyte counts on day 5 (Fig. 9B). Flow cytometric analysis of peripheral blood and BAL fluid on day 5 demonstrated that neutrophils were significantly elevated in both compartments (Fig. 9C and D). This widespread neutrophilia suggested global exacerbation of innate inflammation in $\mathrm{MeCP} 2^{\mathrm{Tg} 3}$ mice on day 5 p.i., that is prior to the onset of the adaptive response in the infected lungs. In agreement with this, Luminex analysis of day 5 BAL fluid revealed a widespread increase in inflammatory cytokines and chemokines (Fig. 10A and Table 1), consistent with infection-induced "cytokine storm" resulting in excess inflammation and enhanced pulmonary injury. Accordingly, we attempted to reduce inflammation via the use of the cycolooxygenase- 2 (COX-2) specific inhibitor, celecoxib. Daily celecoxib treatment normalized neutrophil levels (Fig. 10B), and weight loss was attenuated (Fig. 10C) without any effect on 
survival (Fig. 10D). Importantly, there was no difference in weight loss (Fig. 10C) or neutrophil levels (Fig. 10B) after celecoxib treatment in wild type mice, demonstrating a specific role for excessive inflammation in $\mathrm{MeCP} 2^{\mathrm{Tg} 3}$ mice.

Previous reports, including ours, have implicated $\mathrm{MeCP} 2$ in the control of glucocorticoid response (Braun et al., 2012; Cronk et al., 2015a; Nuber et al., 2005). In addition, $\mathrm{MeCP} 2$ overexpressing mice have been shown to have an enhanced response to stress (Samaco et al., 2012). Therefore, we measured serum corticosterone levels during infection, and found a significant increase starting on day 5 and persisting through day 8 (Fig. 11A). Importantly, glucocorticoids are potent inhibitors of both $\mathrm{T}$ and $\mathrm{B}$ cells (Coutinho and Chapman, 2011), and could explain adaptive immune suppression observed between day 3 and 8 . However, similar to celecoxib treatment, treatment with either the glucocorticoid inhibitor mifepristone or combination treatment with mifepristone and celecoxib resulted in attenuated weight loss (Fig. 11B) but with no effect on $\mathrm{MeCP} 2^{\mathrm{Tg} 3}$ survival after influenza $\mathrm{A}$ infection (Fig. 11C). These results suggest that glucocorticoids play a role in the pathology observed in $\mathrm{MeCP} 2^{\mathrm{Tg} 3}$ mice exposed to influenza A virus, but are insufficient to explain the full extent of disease.

\section{Non-hematopoietic cells are essential for influenza A-induced mortality in}

MeCP2 $^{\mathrm{Tg} 3}$ mice. Based on these cumulative results, we questioned the overall role of hematopoietic cells in explaining influenza A-induced mortality in $\mathrm{MeCP}^{\mathrm{Tg} 3}$ mice. Towards this end, we transplanted wild type or $\mathrm{MeCP}^{\mathrm{Tg} 3}$ mice with either wild type or $\mathrm{MeCP}^{\mathrm{Tg} 3}$ bone marrow and infected with influenza $\mathrm{A} / \mathrm{PR} / 8 / 34$. Survival was not 
improved by wild type bone marrow, nor was survival impaired by $\mathrm{MeCP} 2^{\mathrm{Tg} 3}$ bone marrow, regardless of host genotype (Fig. 12A). This strongly suggested that the immune system alone does not account for mortality in $\mathrm{MeCP} 2^{\mathrm{Tg} 3}$ mice, because the $\mathrm{MeCP} 2^{\mathrm{Tg} 3}$ immune system was insufficient to cause death in wild type hosts, and a wild type immune system was unable to rescue survival in $\mathrm{MeCP} 2^{\mathrm{Tg} 3}$ hosts. Thus, we concluded that non-hematopoietic cells must play an essential role in mortality upon influenza A infection in $\mathrm{MeCP} 2^{\mathrm{Tg} 3}$ mice.

We had previously noted that BAL fluid from infected $\mathrm{MeCP} 2^{\mathrm{Tg} 3}$ mice was bloody suggesting increased vascular leak. When we measured BAL red blood cell (RBC) numbers via flow cytometry on day 5 p.i., we found significantly more RBCs in $\mathrm{BAL}$ fluid of infected $\mathrm{MeCP} 2^{\mathrm{Tg} 3}$ mice (Fig. 12B). In agreement with these findings, we observed that $\mathrm{MeCP} 2^{\mathrm{Tg} 3}$ lungs appeared grossly erythematous on day 7 (Fig. 12C). Analysis of BAL fluid from uninfected, day 5, and day 7 p.i. $\mathrm{MeCP} 2^{\mathrm{Tg} 3}$ mice revealed a progressive increase in airway hemorrhage. By contrast there was no blood evident in BAL fluid from infected wild type mice (Fig. 12D). As an additional measure of vascular leak, Evans Blue dye accumulation was measured in the BAL fluid of infected mice $1 \mathrm{hr}$ after i.p. dye injection. Interestingly, while wild type mice demonstrated Evans Blue in their airways only on day 7 (as would be expected with increasing pulmonary inflammation), some $\mathrm{MeCP} 2^{\mathrm{Tg} 3}$ mice had Evans blue in their airway starting on day 5, but had significantly less than wild type by day 7 (Fig. 12D). This suggested that although airway hemorrhage was occurring on day 5, transport of protein/fluid from the vasculature to the airways was impaired by day 7 p.i. In agreement with this finding, on 
day $7 \mathrm{MeCP} 2^{\mathrm{Tg} 3}$ lungs displayed significant areas of vascular congestion even after pulmonary vascular perfusion with PBS (Fig. 12E). We also found that in contrast to results on day 3 p.i. the number of infiltrating airway cells was significantly reduced in $\mathrm{MeCP}^{\mathrm{Tg} 3} \mathrm{BAL}$ fluid on day 8 , (Fig. $12 \mathrm{~F}$ ), again supporting the possibility that access to the pulmonary vascular compartment is compromised in $\mathrm{MeCP}^{\mathrm{Tg} 3}$ mice after day 5 of infection.

Histologic analysis of lungs from mice day 7 p.i. revealed capillary congestion and alveolar hemorrhage, likely reflecting damage occurring on and after day 5 p.i. (Fig. 13A). Importantly, overall tissue integrity as detected by histology was comparable between wild type and $\mathrm{MeCP} 2^{\mathrm{Tg} 3}$ mice, suggesting that the observed vascular defects represented the major tissue damage leading to death upon infection in $\mathrm{MeCP} 2^{\mathrm{Tg} 3}$ mice. Because we had observed defects of pulmonary perfusion, we decided to measure pulmonary function, which includes pulmonary artery pressure. While resistance and compliance were similar to that of wild type (as expected, since no increased tissue destruction was evident in H\&E stains), pulmonary artery pressure was significantly elevated in $\mathrm{MeCP}^{\mathrm{Tg} 3}$ mice on day 8 (Fig. 12G). H\&E analysis revealed both perivascular edema and pulmonary arterial narrowing in $\mathrm{MeCP}^{\mathrm{Tg} 3}$ mice (Fig. $12 \mathrm{H}$ and $13 \mathrm{~B}$ ), consistent with pulmonary arterial hypertension (Fig. 12G) and inability to effectively perfuse the infected $\mathrm{MeCP} 2^{\mathrm{Tg} 3}$ lungs (Fig. 12E). Interestingly, this vascular abnormality potentially extended to other tissues, as all $\mathrm{MeCP}^{\mathrm{Tg} 3}$ mice had regions of blood in the brain that could not be cleared following perfusion of the brains of $\mathrm{MeCP}^{\mathrm{Tg} 3}$ mice on 
Chapter 4: Lethal outcome and dysfunctional immune response to influenza A in an animal model of MeCP2 overexpression

day 7 p.i. (Fig. 13C), suggesting that severe respiratory infection leads to systemic vascular pathology in the context of $\mathrm{MeCP} 2$ overexpression.

\section{Discussion}

Here we demonstrated that influenza A infection in $\mathrm{MeCP} 2$ overexpressing mice results initially (up to day 3 p.i.) in a normal immune response, but abnormally progresses into excessive innate inflammation, high corticosterone levels, defective adaptive immunity, uncontrolled virus replication, vascular defects, and death approximately 6-9 days post infection. Most pathologic findings began on day 5 post-infection, indicating this as a critical turning point. Much of our data regarding adaptive immune system function agrees with previous reports (Bauer et al., 2015; Koelsch et al., 2013; Yang et al., 2012), and suggests that the adaptive immune response is significantly impaired in the context of MeCP2 overexpression. We have also identified multiple other pathologic processes during infection in $\mathrm{MeCP} 2^{\mathrm{Tg} 3}$ mice, suggesting that the impairment of the adaptive immune response is just one of a complex set of abnormalities occurring during infection in the context of MeCP2 overexpression.

In addition to defects in immune cells, non-hematopoietic cells were also essential to infection-associated lethality. Bone marrow transplant revealed no effect of immune system genotype on mortality, and significant vascular impairment (including perivascular edema, pulmonary arterial hypertension, impaired perfusion, and alveolar hemorrhage) was observed in $\mathrm{MeCP} 2^{\mathrm{Tg} 3}$ mice. These results suggest that either direct response of vasculature, or upstream response by other non-hematopoietic cells, play an 
important role in disease pathogenesis. In order to identify which cell type(s) are responsible for susceptibility to respiratory infection in $\mathrm{MeCP} 2$ overexpressing mice, it would be necessary to either overexpress or correct MeCP2 expression levels in specific non-hematopoietic cell types. Unfortunately, such genetic tools to target specific cell types do not currently exist. Future work should endeavor to both create and utilize cellspecific tools to better understand which pulmonary cell types are critical to mortality associated with respiratory infection in the context of MeCP2 overexpression.

It was previously shown that $\mathrm{MeCP} 2$ overexpressing mice have increased corticosterone release after stress (Samaco et al., 2012), and multiple groups have directly linked MeCP2 to glucocorticoid response (Braun et al., 2012; Cronk et al., 2015a; Nuber et al., 2005), which may explain the increased corticosterone we observed during influenza infection in $\mathrm{MeCP} 2^{\mathrm{Tg} 3}$ mice. In addition, glucocorticoids can suppress the function of and cause apoptosis in lymphocytes (Coutinho and Chapman, 2011), potentially helping to explain the deficiency and impairment of lymphocytes seen late during infection. However, glucocorticoids have a complex effect during infection, as they can both impair the adaptive immune response but also help control innate inflammation. Thus, pharmacologic intervention may be equally complex, as excessive innate inflammation seen in the context of $\mathrm{MeCP} 2$ overexpression may be exacerbated by glucocorticoid blockade. Regardless, the role of $\mathrm{MeCP} 2$ in the glucocorticoid response continues to be an outstanding unresolved question that warrants further investigation.

The excessive innate inflammation seen in this study may correlate to excessive acute phase responses observed in MeCP2 duplication syndrome patients (Bauer et al., 
Chapter 4: Lethal outcome and dysfunctional immune response to influenza A in an animal model of MeCP2 overexpression

2015), and could represent a common defect in the immune response when MeCP2 is overexpressed. Interestingly, significant levels of chemokine (C-X-C motif) ligand 5 (Cxc15) were observed in $\mathrm{MeCP} 2^{\mathrm{Tg} 3} \mathrm{BAL}$ fluid, whereas wild type mice had little to no Cxc15, potentially implicating respiratory epithelial cells in inflammation as these cells are the major known producer of Cxcl5 in the lung (Jeyaseelan et al., 2005; Koltsova and Ley, 2010; Mei et al., 2010; Nouailles et al., 2014). Although inhibition of innate inflammation did not improve survival, we did see symptomatic improvement in the form of normalized neutrophil levels and attenuated weight loss. It is therefore possible that excessive innate inflammation represents a significant cause of morbidity in MeCP2 duplication syndrome, and should be investigated as a potential target for amelioration of symptoms during infection in these patients. This may be of particular interest in the context of less severe infections, where symptomatic treatment may be the most salient consideration.

Our findings could inform future work on respiratory infections in MeCP2 duplication syndrome by suggesting that some potential therapies focusing only on the hematopoietic system, such as bone marrow transplant, may not be viable. Treatment of respiratory infection in $\mathrm{MeCP} 2$ duplication syndrome may require therapies either targeting or genetically correcting non-hematopoietic cells. It was recently shown that antisense oligonucleotides can effectively knockdown MeCP2 expression in MeCP2 overexpressing mice, and ameliorates neurologic symptoms (Sztainberg et al., 2015). A similar strategy could be employed in the context of infection in order to correct nonhematopoietic defects. Such an approach may represent the most promising therapy for 
MeCP2 duplication patients, given our results demonstrating the importance of nonhematopoietic cells in the context of infection.

In sum, this work provides evidence that influenza A infection-related morbidity and mortality in the context of $\mathrm{MeCP} 2$ overexpression is a complex process involving defects of both immune and non-hematopoietic cells. Future studies should endeavor to further understand which cell types drive mortality upon respiratory infection in MeCP2 overexpression, which may lead to targeted therapies for these patients. 
Chapter 4: Lethal outcome and dysfunctional immune response to influenza $A$

\section{Figures and tables}

Table 1.

\begin{tabular}{|c|c|c|c|}
\hline \multicolumn{4}{|c|}{ Luminex of BAL fluid, day 5 post infection } \\
\hline \multirow[b]{2}{*}{ Molecule } & \multicolumn{2}{|c|}{ Concentration (pg/ml \pm SEM) } & \multirow[b]{2}{*}{$p$-value } \\
\hline & Wild type & $\mathrm{MeCP}^{\mathrm{Tg} 3}$ & \\
\hline $\mid \mathrm{IL-17}$ & $1.606 \pm 0.2453$ & $2.630 \pm 0.4033$ & 0.0619 \\
\hline IL-15 & $21.55 \pm 1.742$ & $43.26 \pm 3.210$ & 0.0003 \\
\hline IL-13 & Not detected & Not detected & N/A \\
\hline IL-12p70 & $5.366 \pm 0.5995$ & $11.12 \pm 1.127$ & 0.002 \\
\hline IL-12p40 & $3.412 \pm 0.5398$ & $7.082 \pm 1.236$ & 0.0262 \\
\hline IL-10 & $39.68 \pm 19.36$ & $14.14 \pm 2.959$ & 0.2285 \\
\hline IL-9 & $72.20 \pm 7.842$ & $157.2 \pm 11.89$ & 0.0003 \\
\hline IL-7 & $0.4960 \pm 0.1437$ & $1.682 \pm 0.2160$ & 0.0018 \\
\hline IL-6 & $2058 \pm 186.2$ & $8623 \pm 800.1$ & $<0.0001$ \\
\hline IL-5 & $28.78 \pm 5.473$ & $130.7 \pm 22.27$ & 0.0022 \\
\hline IL-4 & $0.9500 \pm 0.2524$ & $1.764 \pm 0.2816$ & 0.0635 \\
\hline IL-3 & $1.778 \pm 0.2475$ & $3.634 \pm 0.3583$ & 0.0028 \\
\hline IL-2 & $3.292 \pm 0.2591$ & $4.634 \pm 0.2328$ & 0.0049 \\
\hline IL-1 $\beta$ & $21.70 \pm 2.000$ & $44.59 \pm 4.538$ & 0.0017 \\
\hline IL-1 $1 \alpha$ & $10.82 \pm 2.318$ & $26.18 \pm 2.795$ & 0.0029 \\
\hline IFNy & $996.6 \pm 477.5$ & $538.4 \pm 141.8$ & 0.3845 \\
\hline TNFa & $37.81 \pm 2.808$ & $73.43 \pm 7.207$ & 0.0017 \\
\hline CXCL10 & $2770 \pm 405.6$ & $5412 \pm 587.7$ & 0.006 \\
\hline CXCL9 & $2610 \pm 628.5$ & $3485 \pm 250.8$ & 0.2318 \\
\hline CXCL5 & $4.808 \pm 1.805$ & $55.90 \pm 12.97$ & 0.0045 \\
\hline CXCL2 & $131.1 \pm 3.970$ & $174.1 \pm 15.35$ & 0.0267 \\
\hline CXCL1 & $804.9 \pm 60.80$ & $1372 \pm 155.4$ & 0.0094 \\
\hline CCL5 & $48.91 \pm 3.036$ & $100.9 \pm 14.23$ & 0.0073 \\
\hline CCL4 & $643.9 \pm 87.99$ & $1552 \pm 327.5$ & 0.028 \\
\hline CCL3 & $225.2 \pm 12.51$ & $406.9 \pm 30.69$ & 0.0006 \\
\hline CCL2 & $1451 \pm 199.0$ & $4007 \pm 419.2$ & 0.0006 \\
\hline LIF & $182.2 \pm 9.589$ & $1179 \pm 211.6$ & 0.0015 \\
\hline VEGF & $21.99 \pm 5.641$ & $56.67 \pm 6.091$ & 0.0031 \\
\hline Eotaxin & $125.4 \pm 16.57$ & $488.8 \pm 73.97$ & 0.0014 \\
\hline MCSF & $19.48 \pm 1.389$ & $40.43 \pm 3.524$ & 0.0006 \\
\hline GMCSF & $50.18 \pm 5.760$ & $109.4 \pm 12.78$ & 0.0029 \\
\hline GCSF & $1606 \pm 140.2$ & $5473 \pm 752.1$ & 0.001 \\
\hline
\end{tabular}

Luminex analysis of BAL fluid in wild type and $\mathrm{MeCP2}^{\mathrm{Tg} 3}$, day 5 p.i. BAL fluid from wild type and $\mathrm{MeCP} 2^{\mathrm{Tg} 3}$ mice on day 5 pi.i. was assessed for cytokine, chemokine, and growth factor levels. Values are displayed as $\mathrm{pg} / \mathrm{ml}+/-\mathrm{SEM}$. Two-tailed Student's T test was performed for statistical analyses. 
Figure 6.
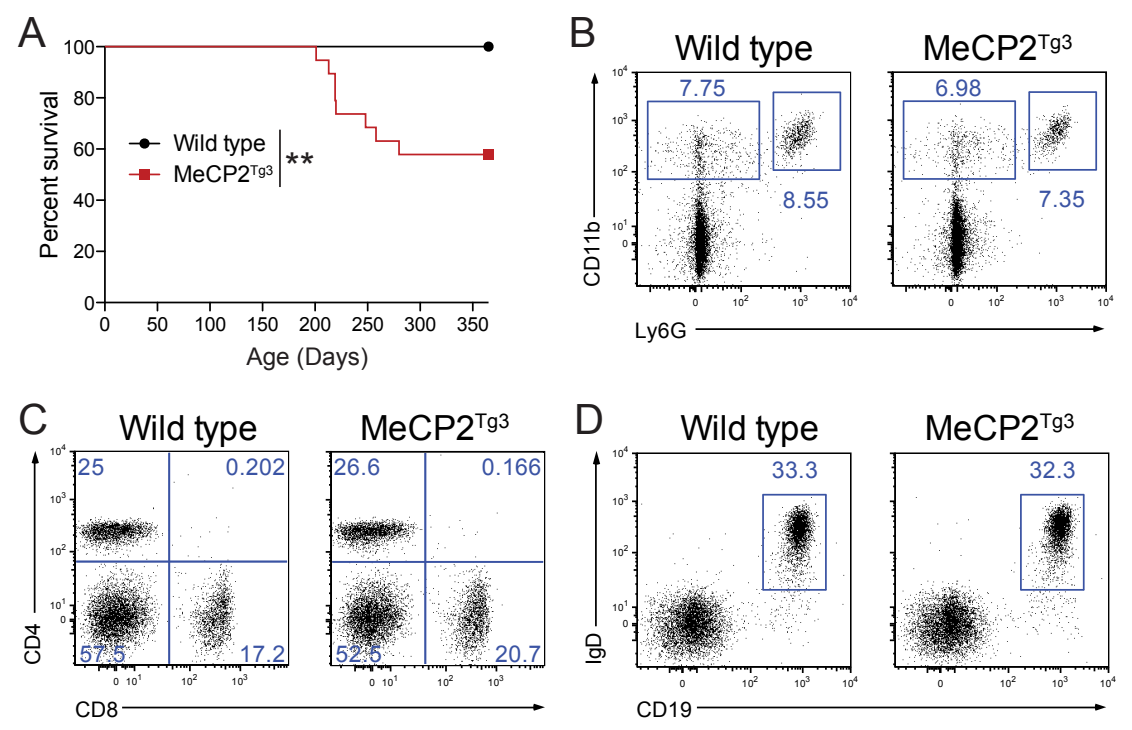

$\mathrm{MeCP2}^{\mathrm{Tg} 3}$ mice naturally start to die after 200 days of age, and have no obvious

baseline peripheral immune defects. (A) Survival curve of C57BL6/J x FVB F1 wild type and $\mathrm{MeCP} 2^{\mathrm{Tg} 3}$ mice ( $\mathrm{n}=20$ wild type, $19 \mathrm{MeCP} 2^{\mathrm{Tg} 3}$ mice, Log-rank (Mantel-cox) test, $\left.{ }^{* *}, \mathrm{p}<0.01\right)$. (B) Representative flow cytometry plots of baseline myeloid cells, gated on live/singlet/CD45+ blood cells. (C) Representative flow cytometry plots of baseline T cells, gated on live/singlet/CD45+ blood cells. (D) Representative flow cytometry plots of baseline B cells, gated on live/singlet/CD45+ blood cells. 
Figure 7.

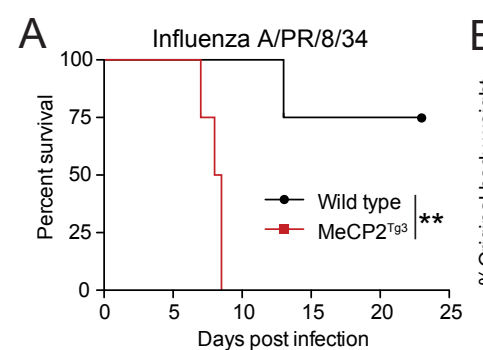

B Influenza A/PR/8/34

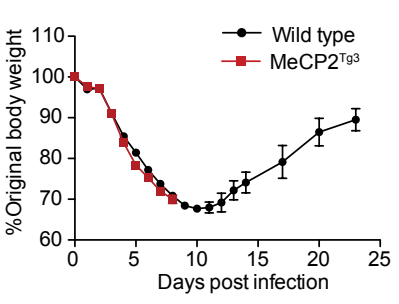

\section{C}
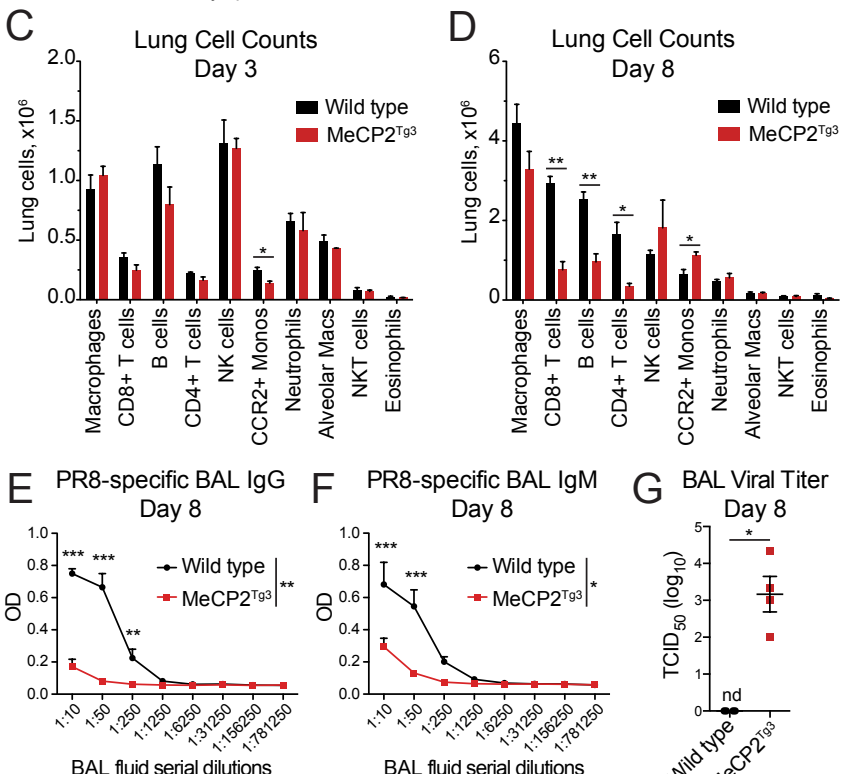

F PR8-specific BAL IgM

G BAL Viral Titer

BAL fluid serial dilutions
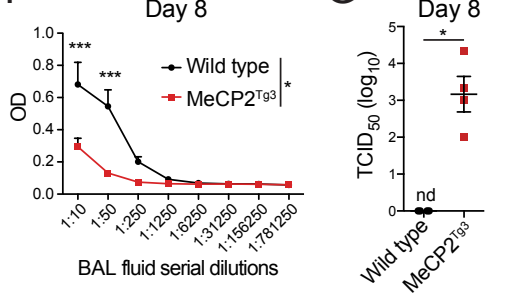
$\mathrm{MeCP2}^{\mathrm{Tg} 3}$ mice are highly susceptible to influenza $\mathrm{A}$ infection, with late adaptive immune failure and uncontrolled virus titers. (A) Survival of 12 week old FVB/C57BL6 F1 male MeCP2 ${ }^{\text {Tg3 }}$ and wild type littermates infected with a dose of influenza $\mathrm{A} / \mathrm{PR} / 8 / 34$ previously determined as $\mathrm{LD}_{100}$ for $\mathrm{MeCP}^{\mathrm{Tg} 3}$ mice $(\mathrm{n}=4$ mice per group, Log-rank (Mantel-Cox) test, ${ }^{* *}, \mathrm{p}=0.0091$ ). (B) Weight loss kinetics as measured by percentage of body weight lost during infection. (C) Lung immune cell counts as measured by flow cytometry on day 3 p.i. ( $\mathrm{n}=3$ mice per group, two-tailed Student's ttest; CCR2+ monocytes, $\mathrm{p}=0.0225$ ). (D) Lung immune cell counts as measured by flow cytometry on day 8 p.i. ( $\mathrm{n}=3$ mice per group, two-tailed Student's t-test for B cells, $\mathrm{p}=0.0051 ; \mathrm{CD} 4+\mathrm{T}$ cells, $\mathrm{p}=0.0155 ; \mathrm{CD} 8+\mathrm{T}$ cells, $\mathrm{p}=0.0013 ; \mathrm{CCR} 2+$ monocytes, $\mathrm{p}=0.0329)$. $(E$ and $F)$ Influenza $\mathrm{A} / \mathrm{PR} / 8$-specific $\operatorname{IgG}$ and $\operatorname{IgM}$ antibody titers as measured by ELISA in BAL fluid from day 8 p.i. ( $\mathrm{n}=3$ mice per group, Two-way ANOVA with Bonferroni post test; *, $\left.\mathrm{p}<0.05, * *, \mathrm{p}<0.01,{ }^{* *}, \mathrm{p}<0.001\right)$. (G) Viral titers from day 8 BAL fluid measured by $\operatorname{TCID}_{50}(\mathrm{n}=4$ mice per group, Mann Whitney test, $\mathrm{p}=0.0211)$. 
Chapter 4: Lethal outcome and dysfunctional immune response to influenza $A$

\section{Figure 8.}

A

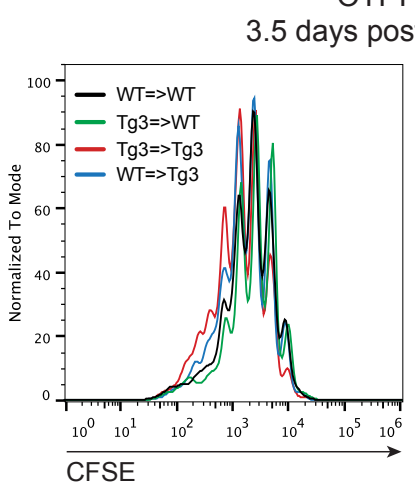

OTI T cell Transfer

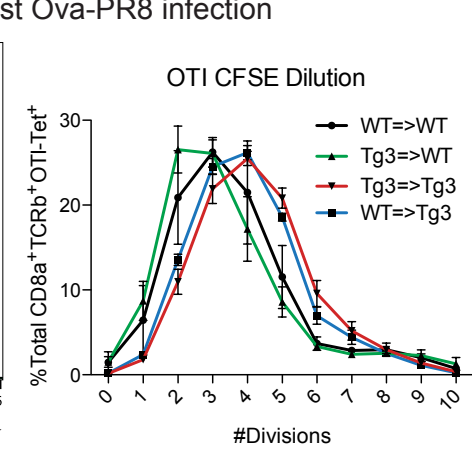

B OTI Avg Divisions
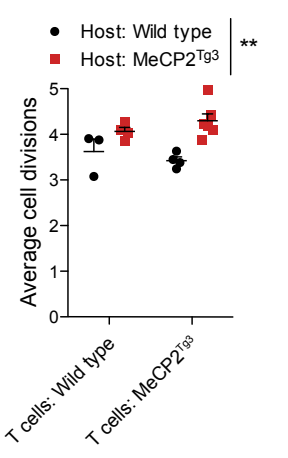

C MLN OTI Count

\begin{tabular}{l|l} 
- Host: Wild type & $* *$ \\
- Host: MeCP2 & (Tg3
\end{tabular}
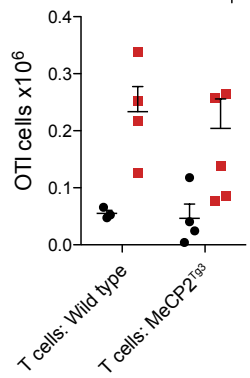

D Lung Day 8, CD45+/Singlets/Live
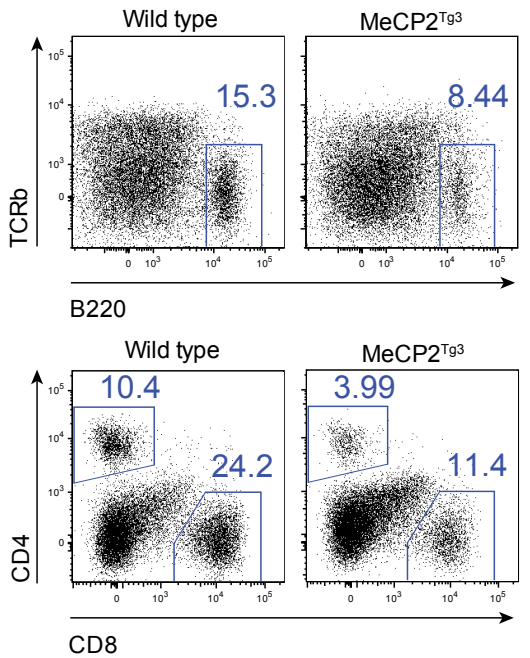

F $\quad$ CD4+ T cells, A/PR8 loaded BMDC re-stimulation

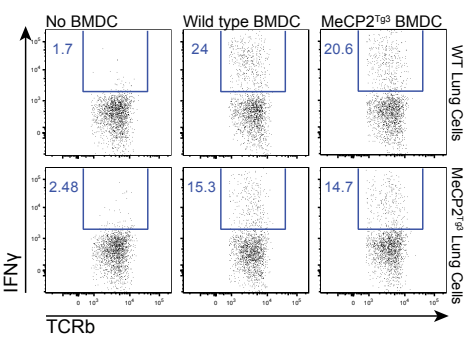

E

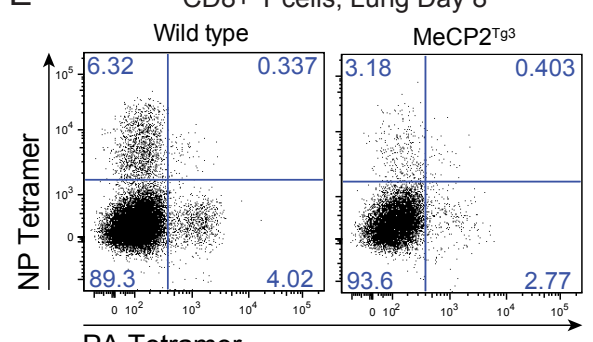

PA Tetramer
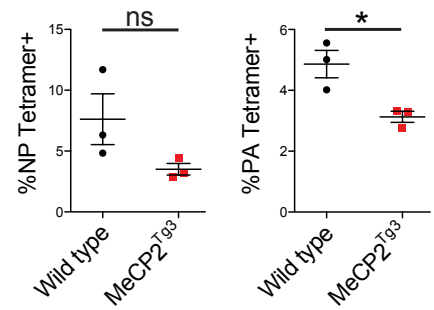

G

CD8+ T cells, A/PR8 loaded BMDC re-stimulation CD45+/Singlets/Live/CD8+/TCRb+

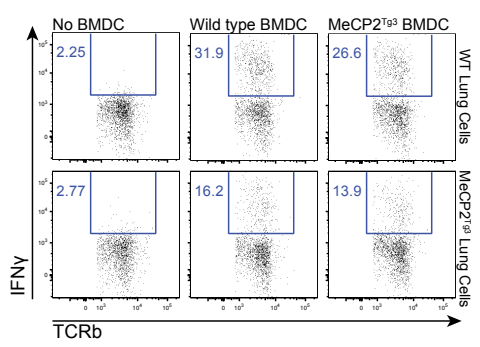

- Wild type

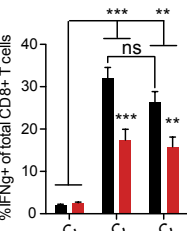

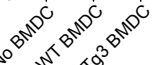


Chapter 4: Lethal outcome and dysfunctional immune response to influenza $A$ in an animal model of MeCP2 overexpression

$\mathrm{MeCP}^{\mathrm{Tg} 3} \mathrm{~T}$ cells display strong activation early during infection, but by day 8 both total and influenza-specific $\mathrm{T}$ cells are deficient, in addition to reduced IFN $\gamma$ production. (A) 2 million $\mathrm{OTI} \mathrm{T}$ cells, either on $\mathrm{MeCP} 2^{\mathrm{Tg} 3}$ or wild type genetic background, were CFSE labeled and transferred into wild type or MeCP2 ${ }^{\mathrm{Tg} 3}$ hosts, which were then infected with OVA-expressing influenza A/PR/8 24 hours later. Mediastinal lymph nodes were collected on day 3.5 post infection and OTI proliferation was analyzed. Histograms of CFSE fluorescence and average number of divisions by OTI T cells are displayed, $\mathrm{n}=3-6$ mice per group. (B) Quantification of average number of divisions by transferred wild type or $\mathrm{MeCP} 2^{\mathrm{Tg} 3}$ OTI $\mathrm{T}$ cells in wild type or $\mathrm{MeCP} 2^{\mathrm{Tg} 3}$ hosts at day 3.5 post infection with OVA-expressing influenza A/PR/8 ( $n=3-6$ mice per group, Two-way ANOVA with Bonferroni post test; **, $\mathrm{p}<0.01) .(C)$ Quantification of wild type or $\mathrm{MeCP} 2^{\mathrm{Tg} 3}$ OTI cell numbers in wild type or MeCP2 ${ }^{\mathrm{Tg} 3}$ hosts at day 3.5 post infection with OVA-expressing influenza A/PR/8 ( $n=3-6$ mice per group, Two-way ANOVA with Bonferroni post test; **, $\mathrm{p}<0.01$ ). (D) Representative flow cytometry plots of $\mathrm{T}$ and $\mathrm{B}$ cells on day 8 post infection. (E) Representative flow cytometry plots and quantification of PA and NP tetramer staining in wild type and $\mathrm{MeCP} 2^{\mathrm{Tg} 3} \mathrm{CD} 8+\mathrm{T}$ cells on day 8 post infection $(n=3$ mice per group, two-tailed Student's t-test; ns, not significant; $\left.{ }^{*}, \mathrm{p}<0.05\right)$. (F) IFN $\gamma$ staining among CD4+ T cells taken from mice day 8 post infection and restimulated with $\mathrm{A} / \mathrm{PR} / 8 / 34$ virus-loaded wild type or $\mathrm{MeCP} 2^{\mathrm{Tg} 3} \mathrm{BMDC}$ ( $\mathrm{n}=3$ mice per group, Two-way ANOVA with Bonferroni post test; **, $\mathrm{p}<0.01, * * *$, $\mathrm{p}<0.001)$. (G) Same as in $(F)$, however CD8+ T cells are displayed ( $\mathrm{n}=3$ mice per group, Two-way ANOVA with Bonferroni post test; * $\left.{ }^{*}<0.05, * *, \mathrm{p}<0.01, * * *, \mathrm{p}<0.001\right)$. 
Chapter 4: Lethal outcome and dysfunctional immune response to influenza $A$ in an animal model of MeCP2 overexpression

Figure 9.

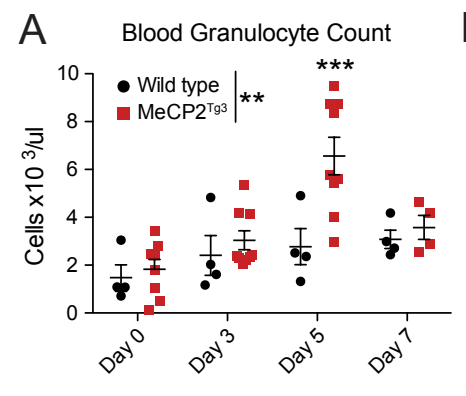

B Blood Granulocyte Count

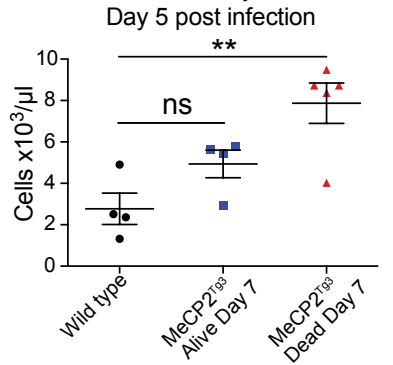

C Blood Neutrophils, Day 5 post infection

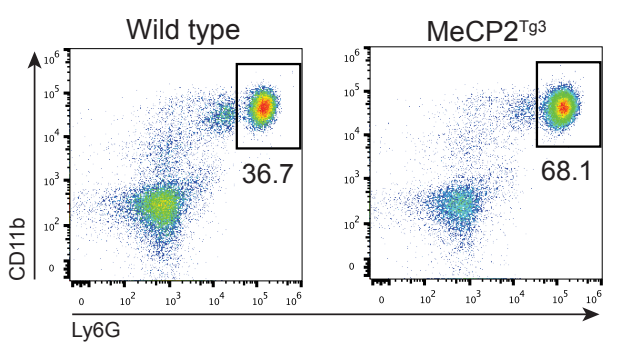

- Wild type - MeCP2 ${ }^{\mathrm{Tg} 3}$
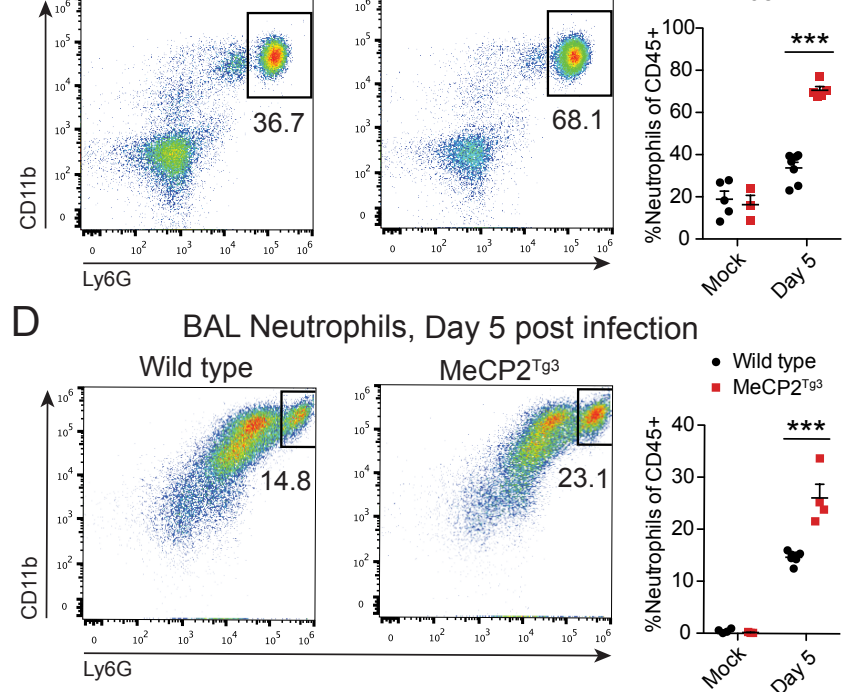

D BAL Neutrophils, Day 5 post infection

- Wild type

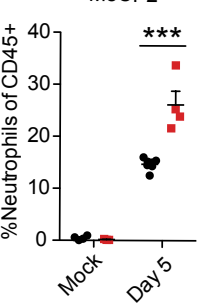


Peripheral and pulmonary neutrophilia associated with mortality occurs on day 5 p.i. in $\mathrm{MeCP2}^{\mathrm{Tg} 3}$ mice. (A) $\mathrm{CBC}$ granulocyte counts from serial tail blood samples taken on days $0,3,5$, and 7 p.i. ( $\mathrm{n}=4$ wild type and $9 \mathrm{MeCP} 2^{\mathrm{Tg} 3}$ mice, Two-way ANOVA with Bonferroni post test; $\left.{ }^{* *}, \mathrm{p}<0.01,{ }^{* *}, \mathrm{p}<0.001\right)$. (B) Comparison of granulocyte count on day 5 p.i. between wild type, $\mathrm{MeCP} 2^{\mathrm{Tg} 3}$ that succumbed prior to day 7 , and $\mathrm{MeCP} 2^{\mathrm{Tg} 3}$ that survived to day 7 (One-way ANOVA with Tukey's multiple comparisons test; **, $\mathrm{p}<0.01)$. (C) Percentage of blood neutrophils out of all live/singlet/CD45+ blood cells on day 5 p.i. ( $\mathrm{n}=3-6$ mice per group, Two-way ANOVA with Bonferroni post test; ***, $\mathrm{p}<0.001$ ). (D) Percentage of BAL neutrophils out of all live/singlet/CD45+ BAL cells on day 5 p.i. ( $\mathrm{n}=3-6$ mice per group, Two-way ANOVA with Bonferroni post test; ***, $\mathrm{p}<0.001)$. 
Chapter 4: Lethal outcome and dysfunctional immune response to influenza $A$ in an animal model of MeCP2 overexpression

Figure 10.
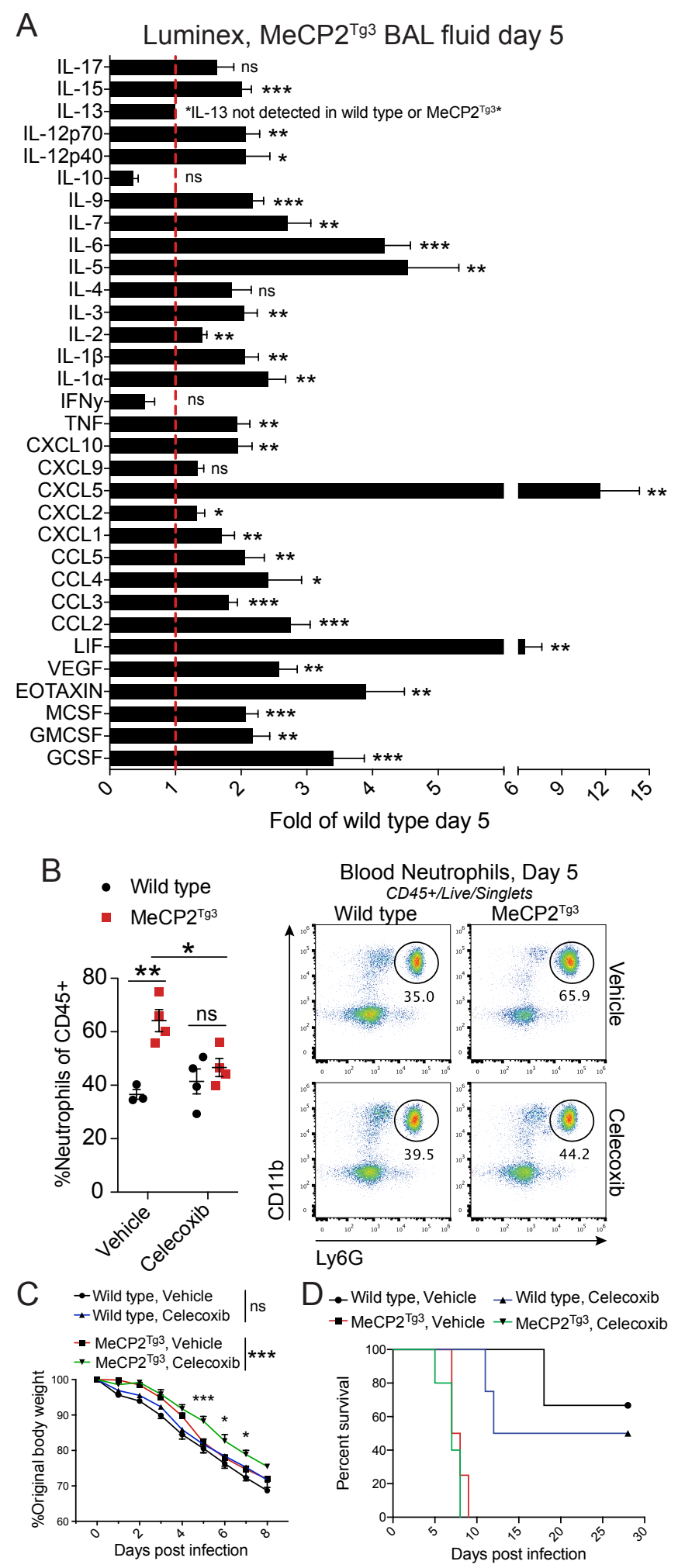
$\mathrm{MeCP}^{\mathrm{Tg} 3}$ mice develop pulmonary cytokine storm, and COX-2 inhibition corrects neutrophilia and reduces weight loss but does not improve survival. (A) Luminex analysis of BAL fluid from day 5 p.i., presented as fold of wild type protein levels $(\mathrm{n}=5$ mice per group, two-tailed Student's t-test; ns, not significant; ${ }^{*}, \mathrm{p}<0.05$; **, $\mathrm{p}<0.01$; ***, $\mathrm{p}<0.001)$. (B) Blood neutrophil percentage out of all live/singlet/CD45+ cells after $25 \mathrm{mg} / \mathrm{kg}$ celecoxib or vehicle treatment on day 5 p.i. ( $\mathrm{n}=3-4$ mice per group, Two-way ANOVA with Bonferroni post test; *, p<0.05, **, p<0.01). (C) Weight loss kinetics during infection with $25 \mathrm{mg} / \mathrm{kg}$ celecoxib treatment $(\mathrm{n}=3-5$ mice per group, Two-way ANOVA with Bonferroni post test; *, $<<0.05$, ***, $\mathrm{p}<0.001$ ). (D) Influenza A/PR/8/34 survival with $25 \mathrm{mg} / \mathrm{kg}$ celecoxib or vehicle treatment $(\mathrm{n}=3-5$ mice per group, Log-rank (Mantel-cox) test, not significant). 
Figure 11.

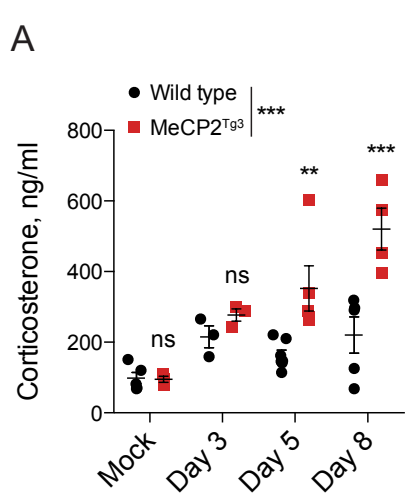

B

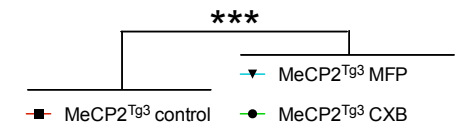

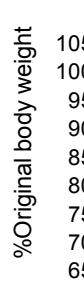

\section{C}

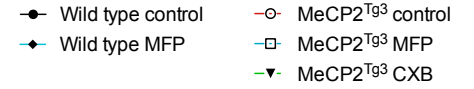

- Wild type MFP+CXB - MeCP2 $2^{T 9}{ }^{3} M F P+C X B$

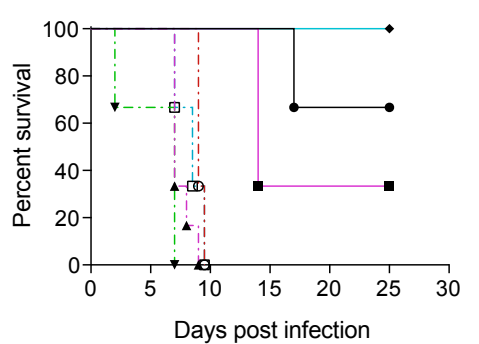

Corticosterone is significantly elevated starting day 5 post infection, however does not explain mortality. (A) Serum corticosterone levels from mice mock infected, day 3 , 5 and 8 post infection. ( $\mathrm{n}=3-7$ mice per group, Two-way ANOVA with Bonferroni post test; **, $\mathrm{p}<0.01, * * *, \mathrm{p}<0.001)$. (B) Weight loss during infection with daily $25 \mathrm{mg} / \mathrm{kg}$ mifepristone (MFP), 100mg/kg celecoxib (CXB), or combination treatment $(\mathrm{MFP}+\mathrm{CXB})$ $(\mathrm{n}=3$ mice per group for all groups except $\mathrm{MFP}+\mathrm{CXB}$, for which $\mathrm{n}=9$, Two-way ANOVA with Bonferroni post test; ***, $\mathrm{p}<0.001$ ). (C) Survival curves for daily $25 \mathrm{mg} / \mathrm{kg}$ mifepristone (MFP), 100mg/kg celecoxib (CXB), or combination treatment $(\mathrm{MFP}+\mathrm{CXB})$ in wild type and $\mathrm{MeCP} 2^{\mathrm{Tg} 3}$ mice ( $\mathrm{n}=3-6$ mice per group). 


\section{Figure 12.}

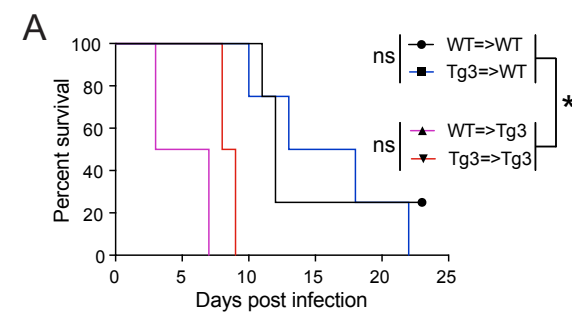

B BAL RBCs, Day 5 post infection
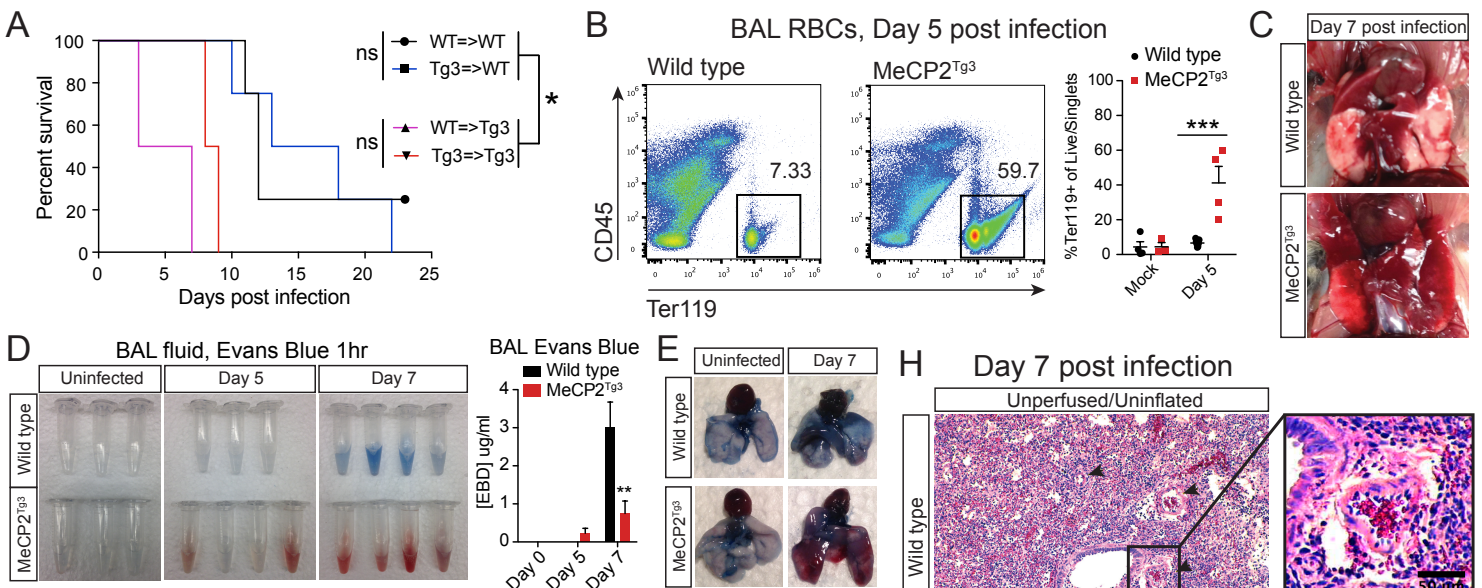

BAL Evans Blue
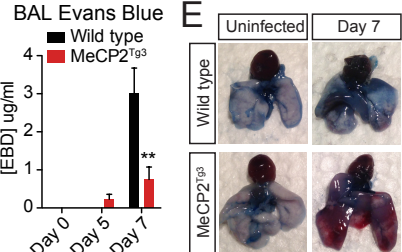

F BAL Cell Count G
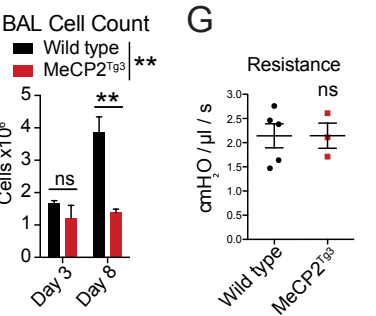

Day 8 post infection

Compliance

H Day 7 post infection
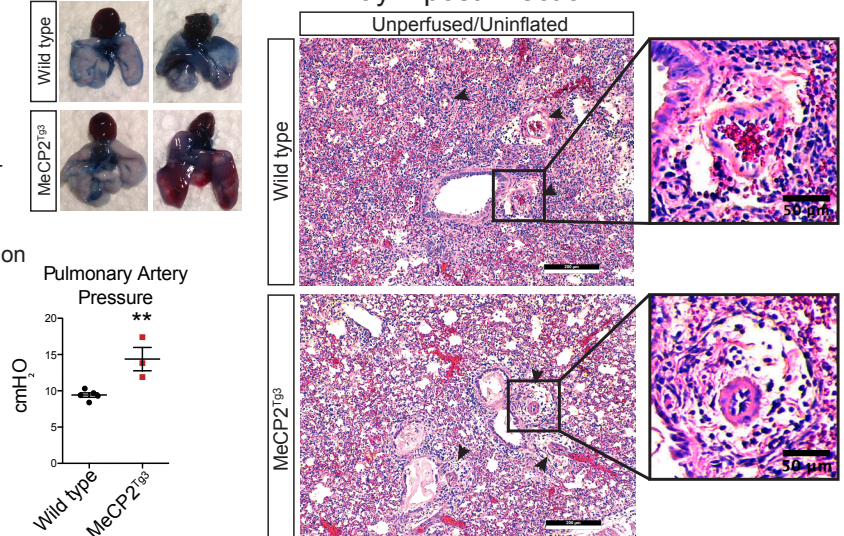


\section{The $\mathrm{MeCP2}^{\mathrm{Tg} 3}$ immune system is insufficient to cause enhanced mortality to} influenza A infection, and influenza A infection in $\mathrm{MeCP}^{\mathrm{Tg} 3}$ mice leads to vascular pathology. (A) Survival curve for mice infected with Influenza A/PR/8/34 that were given bone marrow transplant with $\mathrm{MeCP} 2^{\mathrm{Tg} 3}$ or wild type bone marrow $(\mathrm{n}=3$ or 4 mice per group for wild type hosts, and 2 mice per group for $\mathrm{MeCP} 2^{\mathrm{Tg} 3}$ hosts, Log-rank (Mantel-cox) test, *, p<0.05). (B) Flow cytometry of RBCs in BAL fluid on day 5 p.i. ( $\mathrm{n}=3-6$ mice per group, Two-way ANOVA with Bonferroni post test; ***, $\mathrm{p}<0.001)$. (C) Gross appearance of wild type and $\mathrm{MeCP} 2^{\mathrm{Tg} 3}$ lungs on day 7 p.i. (D) Images and quantification of Evans Blue in BAL fluid taken from uninfected, day 5, and day 7 p.i. Mice were injected with Evans Blue dye one hour prior to harvest ( $n=3-5$ mice per group, Two-way ANOVA with Bonferroni post test; **, p<0.01). (E) Gross appearance of lungs from mice given Evans Blue dye and analyzed one hour later. Lungs were perfused to remove remaining vascular dye and blood, and BAL fluid was taken. Images are representative of $n=3$ mice per group for uninfected, and $n=4$ mice per group for day 7 p.i.. (F) Total BAL cell counts on day 3 and day 8 p.i. (n=3 mice per group, Two-way ANOVA with Bonferroni post test; ns, not significant; **, $<<0.01$ ). (G) Resistance, compliance, and pulmonary artery pressure measured on day 8 p.i. ( $n=5$ for wild type and 3 for $\mathrm{MeCP} 2^{\mathrm{Tg} 3}$, two-tailed Student's t-test, $\mathrm{p}=0.0074$ for pulmonary artery pressure). $(H)$ H\&E staining of day 7 p.i. lungs that were not perfused or inflated demonstrates both arterial narrowing and perivascular edema (black arrowheads, pulmonary arteries). Scale bar $=200 \mu \mathrm{m}$ for large images, and $50 \mu \mathrm{m}$ for insets. 


\section{Figure 13.}
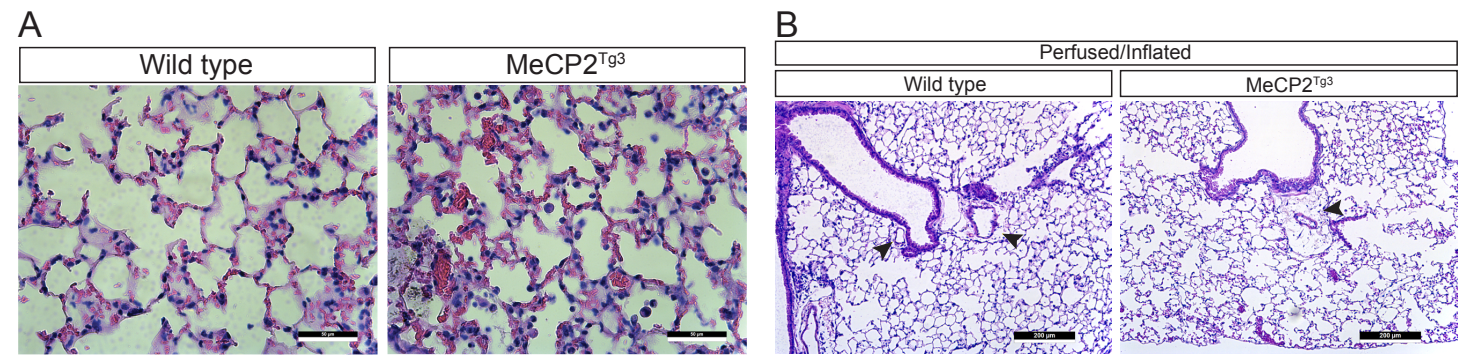

C
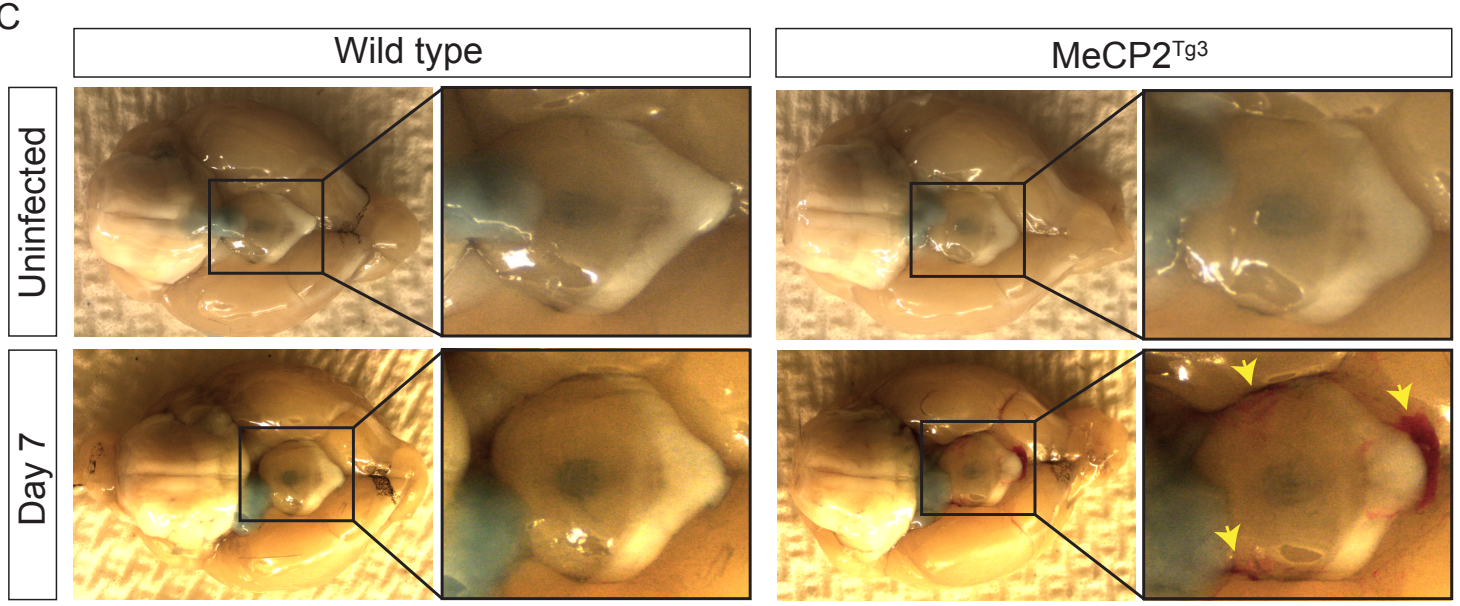

Vascular pathology at day 7 post infection in MeCP2 $^{\mathrm{Tg} 3}$ mice. $(A)$ H\&E staining of

day 7 post infection lungs that were not perfused or inflated demonstrates capillary congestion and alveolar hemorrhage in $\mathrm{MeCP} 2^{\mathrm{Tg} 3}$ mice. Scale bar $=50 \mu \mathrm{m}$. $(B) \mathrm{H} \& \mathrm{E}$ staining of day 7 post infection lungs that were perfused and inflated demonstrates both arterial narrowing and perivascular edema (black arrowheads, pulmonary arteries). Scale bar $=200 \mu \mathrm{m} .(C)$ Evidence of hemorrhage in the brains of day 7 post infection $\mathrm{MeCP} 2^{\mathrm{Tg} 3}$ mice (yellow arrowheads). Evans Blue dye injected mice were systemically perfused to remove blood and remaining vascular dye. Images are representative of $n=3$ mice per group for uninfected, and $n=4$ mice per group for day 7 post infection. 


\section{Chapter 5}

\section{Summary discussion and future directions}

\section{A reassessment of $\mathrm{MeCP} 2$ in health and disease}

$\mathrm{MeCP} 2$ is a complex regulator of transcriptional responses. It has been shown to interact with a plethora of molecules in the control of gene transcription, and is both a repressor and activator of gene transcription (Guy et al., 2011). In addition, its loss- or gain-offunction results in widespread, relatively small transcriptional changes (Ben-Shachar et al., 2009; Tudor et al., 2002). Indeed, the scientific community is still discovering novel, fundamental functions for $\mathrm{MeCP} 2$, such as new DNA binding motifs beyond $5 \mathrm{mC}$ (Mellen et al., 2012). Given the complexity of understanding MeCP2, work to explain how it might regulate cellular function on a basic level, much less how malfunction of MeCP2 might mediate disease, has been slow, and far too often, incomplete or 
inconclusive. This frustration has led to attempts by the scientific community to simplify the concepts and biological processes being examined by focusing only on single organ systems (the CNS) and cell types (neurons) (Chahrour and Zoghbi, 2007; Guy et al., 2011; Lombardi et al., 2015). While such an approach can be helpful in making progress towards understanding a highly complex system, in this case a failure to consider the pleiotropic roles for $\mathrm{MeCP} 2$ may instead lead to further difficulties in understanding disease pathogenesis of MeCP2-related pathologies. At worst, this closed mindset could even lead to misguided conclusions based on incorrect presumptions regarding the roles and functions of $\mathrm{MeCP} 2$.

The pathogenesis of Rett and MeCP2 duplication syndromes has long been assumed as an essentially neuronal process (Chahrour and Zoghbi, 2007; Guy et al., 2011; Lombardi et al., 2015). Even in the case of MeCP2 duplication syndrome, in which chronic, life-threatening infections are a recognized feature of the disease, the direct role of $\mathrm{MeCP} 2$ in causing immunologic pathology was initially questioned (Ramocki et al., 2010). It was thought that a neighboring gene, encoding interleukin-1 receptor-associated kinase 1 (IRAK1) may be responsible for immunologic aspects of $\mathrm{MeCP} 2$ duplication syndrome, because it is frequently duplicated along with $M e C P 2$ (Ramocki et al., 2010). However, studies using mice that specifically overexpress only $M e C P 2$, and not $I R A K 1$, have demonstrated that $\mathrm{MeCP} 2$ significantly influences immune function on its own (Koelsch et al., 2013; Yang et al., 2012). While it is entirely possible that IRAKI duplication contributes to immune failures in $\mathrm{MeCP} 2$ duplication syndrome, recent evidence, including the results presented in this dissertation, demonstrates that MeCP2 
plays a direct role in not only the immune function in $\mathrm{MeCP} 2$ duplication syndrome (Koelsch et al., 2013; Yang et al., 2012), but also MeCP2 loss-of-function / Rett syndrome (Cronk et al., 2015a; Cronk et al., 2015b; Derecki et al., 2012; Horiuchi et al., 2016; Jiang et al., 2014; Jin et al., 2015; Li et al., 2014; Maezawa and Jin, 2010; O'Driscoll et al., 2013b; O'Driscoll et al., 2015), and potentially even other immunologic disorders outside of either Rett or MeCP2 duplication (Carmona et al., 2013; Cobb et al., 2010; Han et al., 2013; Kaufman et al., 2013; Koelsch et al., 2013; Sawalha et al., 2008; Webb et al., 2009).

The immune system expresses and utilizes MeCP2 within many cell types. Evidence for this from both our lab and others has been accumulating in recent years (Cronk et al., 2015a; Cronk et al., 2015b; Derecki et al., 2012; Horiuchi et al., 2016; Jiang et al., 2014; Jin et al., 2015; Li et al., 2014; Maezawa and Jin, 2010; O'Driscoll et al., 2013b; O'Driscoll et al., 2015). In this body of work, we have demonstrated that MeCP2 plays important roles for immune homeostasis and response in multiple contexts. First, we showed that macrophages, including microglia, utilize Mecp2 for control of transcriptional responses to stimuli (Chapter 3, Appendix). Second, we investigated the pathogenesis of respiratory infection in a mouse model of $\mathrm{MeCP} 2$ duplication syndrome, elucidating dysfunction of both immune and non-hematopoietic cells during influenza A infection (Chapter 4). These results, combined with the mounting body of published evidence, make clear that $\mathrm{MeCP} 2$ is required for proper function of the immune system. 


\section{Regulation of responses to stimuli in immune cells by $\mathrm{MeCP} 2$}

Given the results of these studies, it can be concluded that MeCP2 is playing cell-intrinsic roles in immune functions. We demonstrated that Mecp2 regulates multiple stimulusinduced transcription pathways in microglia and macrophages by both measuring their abnormal expression in vivo, and their abnormal expression in vitro (Cronk et al., 2015a). Thus, we verified that Mecp2 is required for control of macrophage transcriptional responses in the context of disease, and that Mecp2 regulates macrophages in a cellintrinsic manner (Cronk et al., 2015a). Others have also measured cell-intrinsic roles for Mecp2 in microglia (Jin et al., 2015; Maezawa and Jin, 2010) and other myeloid cells (O'Driscoll et al., 2013b; O'Driscoll et al., 2015). One of our key findings was that Mecp2 acts as a transcriptional repressor of inflammatory responses upon Tnf signaling in macrophages (Cronk et al., 2015a); a finding that another group replicated in other myeloid cells shortly after we published our results (O'Driscoll et al., 2015). Beyond macrophage response to stimuli, it is also becoming clear that $\mathrm{MeCP} 2$ regulates $\mathrm{T}$ cell skewing and subsequent cytokine production (Jiang et al., 2014; Li et al., 2014; Yang et al., 2012), and may play a role in antibody production and/or class switching (Bauer et al., 2015; Koelsch et al., 2013).

In addition to inflammatory transcriptional responses, we also found that Mecp2 regulates glucocorticoid signaling in macrophages (Cronk et al., 2015a). This was not an entirely surprising finding, as $\mathrm{MeCP} 2$ had been previously demonstrated to regulate glucocorticoid signaling and bind to the promoters of key glucocorticoid response genes (Braun et al., 2012; Nuber et al., 2005). This finding becomes more interesting when 
considered alongside our observation that $\mathrm{MeCP} 2^{\mathrm{Tg} 3}$ mice experienced large elevations in circulating glucocorticoids during infection (Fig. 11). Precedent also existed for this finding, as it had been shown that MeCP2 overexpressing mice have an exaggerated stress response, including increased corticosterone release (Samaco et al., 2012). The evidence thus shows that $\mathrm{MeCP} 2$ regulates glucocorticoid responses in multiple cell types, and that both overexpression and deletion of Mecp2 leads to perturbation of the glucocorticoid system. Therefore, a global role for $\mathrm{MeCP} 2$ may be the control of response to, and release of, glucocorticoids. If this is the case, then it may be expected that abnormalities of the glucocorticoid system will be a common feature of any MeCP2 disorder. This could have complex downstream ramifications, as glucocorticoids have potent effects in a multitude of body systems, especially the immune system (Coutinho and Chapman, 2011). Glucocorticoids are one of the strongest anti-inflammatory drugs used in the clinic, capable of both dampening inflammatory responses and even inducing apoptosis of immune cells (Coutinho and Chapman, 2011). Therefore, if MeCP2 is a central regulator of glucocorticoid signaling, then this may represent a core function for $\mathrm{MeCP} 2$ in the immune system.

Given that MeCP2 regulates glucocorticoid responses across cell types (Braun et al., 2012; Cronk et al., 2015a; Nuber et al., 2005), MeCP2 may regulate other common pathways. As an example, Tnf has been shown to regulate sensory neuron development (Wheeler et al., 2014) and synaptic scaling (Stellwagen and Malenka, 2006). Thus, if MeCP2 regulates neuronal Tnf signaling, as it does in macrophages/myeloid cells (Cronk et al., 2015a; O'Driscoll et al., 2015), it may represent yet another pathway that is 
commonly regulated by $\mathrm{MeCP} 2$ in very different cells. MeCP2 may in fact function to regulate common pathways throughout the body, but the downstream effects due to dysregulation of the same pathway in different cell types may result in unique, cell-type specific outcomes. Thus, common molecular etiologies of MeCP2 dysfunction may remain elusive based on phenotypes alone, as the phenotypic effects of signaling abnormalities in the same pathways within neurons and immune cells may be entirely different, regardless of commonalities in molecular etiology. As an example, enhanced Tnf signaling in immune cells might result in excessive inflammatory responses (Cronk et al., 2015a; O'Driscoll et al., 2015), whereas enhanced Tnf signaling in neurons might result in abnormal neurodevelopmental outcomes (Stellwagen and Malenka, 2006; Wheeler et al., 2014). In isolation, no clear link between these phenotypes would be evident, thus investigation of the underlying molecular etiologies would be key to realizing a common cell-intrinsic causation.

Another layer of complexity can be found in our observation that expression of $\mathrm{MeCP} 2$ is dynamically regulated upon inflammatory stimulation (Fig. 5). We found that LPS stimulation not only increases Mecp2 expression 3-4 fold in macrophages, but that IFN $\gamma$ priming attenuates this response. Interestingly, it was found that TNF induces $\mathrm{MeCP} 2$ expression in an embryonic cell line upon neuronal differentiation, suggesting such induction of $\mathrm{MeCP} 2$ by inflammatory signaling may also cross cell type boundaries (O'Driscoll et al., 2013a). This suggests that expression of MeCP2 itself is dynamically regulated in macrophages, and potentially other cells. Dynamic regulation of MeCP2 
levels within a cell may therefore represent an additional layer of transcriptional control, superimposed upon the direct effects of $\mathrm{MeCP} 2$ on gene expression.

A model including dynamic control of MeCP2 expression may help to explain why, for instance, MeCP2 duplication syndrome results in such a devastating disease (Ramocki et al., 2010). If MeCP2 literally binds every methylated $\mathrm{CpG}$ at all times in the normal condition, then $\mathrm{MeCP} 2$ duplication should not result in disease, as excess protein should have no appreciable effect. However, given the significant pathology induced by MeCP2 overexpression (Alvarez-Saavedra et al., 2010; Bodda et al., 2013; Collins et al., 2004; Friez et al., 2006; Koelsch et al., 2013; Ramocki et al., 2010; Samaco et al., 2012; Yang et al., 2012), the idea that MeCP2 always binds every methylated CpG seems increasingly unlikely. Tightly controlled, dynamic expression of $\mathrm{MeCP} 2$ better fits the observed phenotypes. The expression patterns of MeCP2 during development also support this concept, as $\mathrm{MeCP} 2$ is progressively expressed in a temporal manner (Balmer et al., 2003; Kishi and Macklis, 2004). Additional studies are needed to better understand how MeCP2 expression might be dynamically regulated within cells, and what stimuli regulate its expression.

In sum, the combined evidence builds a case for $\mathrm{MeCP} 2$ as a broad regulator of stimulus response in immune cells. Although researchers have analyzed different cell populations, stimuli, and experimental models, all have found in common that response to inflammatory stimuli is affected when MeCP2 function is altered (Cronk et al., 2015a; Jiang et al., 2014; Li et al., 2014; O'Driscoll et al., 2013b; O'Driscoll et al., 2015; Yang et al., 2012). With this in mind, understanding how MeCP2 will regulate an immune 
response becomes a complex question. This is for three reasons: 1) multiple populations of disparate immune cell types will be simultaneously affected by $\mathrm{MeCP} 2$ perturbation on a cell-intrinsic level upon initiation of an immune response; 2) the utilization of MeCP2 by multiple disparate cell types makes determination of cell-intrinsic effects versus downstream cell-extrinsic effects difficult; and 3) it may not be only immune cells, but indeed non-hematopoietic cells that also experience MeCP2-mediated transcriptional regulation of their response to immunologic insult. This emerging understanding of where $\mathrm{MeCP} 2$ fits into a functional immune system suggests that there is much work to do if we are to understand both the role of $\mathrm{MeCP} 2$ on a per-cell basis, and also how those cell-intrinsic functions relate to the complex, multi-cellular events comprising an inflammatory immune response.

\section{A complex network of cell-intrinsic and extrinsic functions}

Cell-intrinsic functions can be classically claimed via isolation of a cell from its environment, or targeted genetic manipulation of a single cell type. However, understanding the relative cell-extrinsic effects of a genetic disease affecting many cell types in many tissues (such as Rett or MeCP2 duplication syndromes) is not such a straightforward endeavor.

The complexity inherent to studying Rett and MeCP2 duplication syndromes partly stems from the fact that $\mathrm{MeCP} 2$ is expressed in cells throughout the body, and therefore likely plays a role in many functions, depending on the cell type being considered (Alvarez-Saavedra et al., 2010; Ballas et al., 2009; Blue et al., 2015; 
Buchovecky et al., 2013; Conti et al., 2015; Cronk et al., 2015a; Cronk et al., 2015b; Gold et al., 2014; Jiang et al., 2014; Li et al., 2014; Lioy et al., 2011; Nguyen et al., 2013; O'Connor et al., 2009; Song et al., 2014; Yang et al., 2012). Therefore, it is likely that given any in vivo scenario, one could measure a complex network of cell-intrinsic effects of $\mathrm{MeCP} 2$ perturbation, which in turn lead to cell-extrinsic effects, which then result in additional cell-intrinsic effects, and so on. In other words, the response of nearly every MeCP2-mutant cell type may be affected on a cell-intrinsic level, thus altering nearly every cell-cell interaction, and resulting in a cumulative "snowball" effect on the wholeorganism level. Even if MeCP2 perturbation is isolated to a specific cell type, this only answers the question as to whether or not MeCP2 is utilized by that specific cell in a cellintrinsic manner. Such evidence does not predict how a cell will respond in the context of a whole-organism abnormality of $\mathrm{MeCP} 2$ expression. There may in fact be phenotypes that are not unmasked without the occurrence of upstream events. For example, Mecp2null macrophages are unlikely to induce any pathology until they experience a stimulus, such as Tnf or glucocorticoids. Such phenotypes would be "silent" until the correct context was present.

Based on our data, and the work of others, it is likely that such a "snowball" effect is indeed initiated by the loss of MeCP2 in neurons (Chen et al., 2001; Cronk et al., 2015a; Cronk et al., 2015b; Giacometti et al., 2007; Guy et al., 2011; Guy et al., 2001; Luikenhuis et al., 2004). This does not indicate, however, that MeCP2 is irrelevant in other cell types. Instead, it implicates neurons as central drivers of pathologies that span the entire organism. For instance, dysfunctional stress responses of neurogenic origin 
might lead to increased circulating glucocorticoids. MeCP2-mutant immune cells, or other non-neuronal cell types, might in turn respond improperly to the excessive release of glucocorticoids. Their improper response could then feed back to neurons, driving additional neurologic pathology. In such a model, non-neuronal cells would act as “amplifiers" of pathology, while neurons act as initiators (Fig. 14) (Cronk et al., 2015b). Thus, correction of MeCP2 expression in non-neuronal cell types might ameliorate symptoms by blunting amplification of disease. Replacement or correction of nonneuronal cells via transplant is a much more realistic prospect than correction of neuronal MeCP2 expression given currently available tools and technology. Therefore, while correction of non-neuronal cells is unlikely to fully rescue disease, it may represent one of the most realistic near-term treatment strategies for patients.

\section{MeCP2 as a multifunctional orchestrator of immune response}

Our results regarding influenza $\mathrm{A}$ infection in the context of $\mathrm{MeCP} 2$ overexpression also suggest a network of cell-intrinsic and extrinsic effects for MeCP2. While we saw significant increases in innate inflammation, neutrophilia, and a defective adaptive immune response, bone marrow transplant failed to indicate that hematopoietic cells alone lead to infection-related mortality (Fig. 12). In addition, differences in early antigen-specific $\mathrm{T}$ cell responses were driven by the genotype of the host, and not the genotype of the T cells (Fig. 8). However, others have measured a cell-intrinsic defect of IFN $\gamma$ production by MeCP2 overexpressing T cells (Yang et al., 2012), a finding that our data supports (Fig. 8). In addition, data indicated that vascular failures may account for 
the major non-hematopoietic contribution to mortality (Fig. 12 and 13), and molecular analysis of BAL fluid during infection revealed a signature implicating respiratory epithelial cells via a fold-increase in Cxc15 well above wild type levels, and far greater than any other molecule analyzed (Fig. 10 and Table 1). Together, these results paint a picture of combined failures in both non-hematopoietic and immune cell responses to infection, which cumulatively create the overall failure to clear and resolve infection and inflammation (Fig. 15).

The observed vascular defects during infection could result from multiple potential sources. The first potential explanation is primary vasculature dysfunction. Second, neurogenic failure to properly regulate vascular tone could contribute to the increased pulmonary artery pressure and luminal narrowing we observed. Third, vasoconstriction may be directly induced by hypoxia in the lungs (Weir and Archer, 1995). Regardless of the etiology, the vasculature is critical for normal immune function, as it serves as both the route by which immune cells travel to an inflamed site and a direct signaling partner to immune cells (Biedermann, 2001; Butcher and Picker, 1996; CookMills and Deem, 2005; Danese et al., 2007). Without proper vascular response to inflammation, a normal immune response cannot take place. Thus, regulation of the vascular response to immunologic insult may represent an additional way in which MeCP2 orchestrates immune responses, outside of hematopoietic cells themselves. Thus, the role of $\mathrm{MeCP} 2$ in vascular function is an important area for future investigation.

One indication of cell-extrinsic effects during influenza A infection in $\mathrm{MeCP} 2^{\mathrm{Tg} 3}$ mice was the fact that anti-inflammatory treatment corrected neutrophilia and reduced 
weight loss in $\mathrm{MeCP}^{\mathrm{Tg} 3}$ mice (Fig. 10), thus suggesting that neutrophilia is driven by inflammation, and is not a primary neutrophil defect. While it did not prevent mortality, anti-inflammatory treatment may represent a potential avenue for symptom management in patients with MeCP2 duplication syndrome if similar excessive innate inflammation is confirmed in the human disease.

Interestingly, we also observed neutrophilia in Mecp2-null mice (Cronk et al., 2015a); whether or not these are interraleted phenotypes between MeCP2-overexpressing and Mecp2-null mice remains to be determined. However, neutrophilia in Mecp2-null mice might represent a relatively uncomplicated, truly cell-extrinsic effect of Mecp2 lossof-function. We found that neutrophils were one of the only immune cell types we measured which do not express detectable levels of Mecp2 (Cronk et al., 2015a). Therefore, any changes in neutrophils were expected to be in response to extrinsic factors. We found that loss of Mecp2 in macrophages directly led to increased Gcsf production upon stimulation. By neutralizing Gcsf in Mecp2-null mice, we were able to not only correct neutrophilia, but also moderately improve lifespan (Cronk et al., 2015a). This may represent at least one example of a straightforward cell-intrinsic dysfunction (Gcsf overexpression) leading to a cell-extrinsic pathology (neutrophilia), which can be corrected by direct inhibition of a single molecular defect (Gcsf neutralization). While most pathology in the context of MeCP2 loss-of-function is likely much more complex, this gives hope that at least some disease phenotypes might be corrected by simple interventions. 
The loss of microglia and macrophages throughout Mecp2-null mice with disease progression also represents a significant failure of immune homeostasis. The exact cause of this phenomenon remains unknown, however it was suggested by others that impaired glutamine/glutamate metabolism and ROS production may be involved (Jin et al., 2015). Based on our findings that Mecp2 primarily functions to regulate response to stimuli in macrophages (Cronk et al., 2015a), it is likely that these phenotypes are primarily driven by some aberrant stimulus response due to the lack of Mecp2 in macrophages. This is an outstanding avenue for research that should be pursued in future work.

\section{MeCP2 and the immune system in human disease}

While our findings in mouse models of MeCP2 loss- and gain-of-function demonstrated an important role for $\mathrm{MeCP} 2$ in regulation of immune function, the question still remains as to how this may relate to human health and disease. Interestingly, one group recently reported that both patients and mice with $\mathrm{MeCP} 2$ loss-of-function demonstrate an inflammatory lung process upon radiologic or histologic examination of the lungs (De Felice et al., 2014). This points to the possibility that immunologic disease processes may be occuring in Rett syndrome, and further raises the possibility that the lungs are an important site of immune regulation by $\mathrm{MeCP} 2$, as was demonstrated in Chapter 4. In addition, it lends support to our findings that Mecp2 loss-of-function leads to excessive inflammatory responses (Cronk et al., 2015a). In order to further elucidate the possibility of aberrant inflammatory response in Rett syndrome, a formal study of the response by 
Rett patients to infection/inflammation would be necessary. Such a study may help towards understanding how the immune system may be contributing to Rett syndrome.

Rett syndrome patients in general may experience symptoms related to immunebased dysfunction, however their inability to effectively communicate may represent a major hurdle towards the acknowledgement of their existence. In MeCP2 duplication syndrome, the immunologic defects are impossible to miss, because they represent an obvious, life-threatening aspect of disease (Bauer et al., 2015; Friez et al., 2006; Ramocki et al., 2010; Yang et al., 2012). These immunologic aspects of MeCP2 duplication syndrome also represent clear evidence that $\mathrm{MeCP} 2$ is, in fact, critical to normal immune function. However, patients with Rett syndrome may suffer from more subtle issues, such as chronic inflammatory disease. Indeed, Rett syndrome patients experience frequent bowel complications (Motil et al., 2012), which in theory could be related to a chronic inflammatory process. Additionally, Rett syndrome patients experience significant bone pathology, including scoliosis and osteopenia (Chahrour and Zoghbi, 2007; Motil et al., 2014). It was recently shown that osteoblasts, the cells responsible for creating bone, are impaired in the absence of Mecp2 (Blue et al., 2015), and thus their failure to properly build bone may contribute to bone pathology in Rett syndrome. Immune cells are also key to the regulation of bone mass; osteoclasts are the major cells responsible for homeostatic bone breakdown, and are formed via the fusion of multiple macrophages (Teitelbaum, 2000). Thus, based on our findings that Mecp2 directly regulates transcriptional response in macrophages (Cronk et al., 2015a), it is entirely possible that 
aberrant transcriptional responses by $\mathrm{MeCP} 2$-mutant osteoclasts may result in excessive bone breakdown in Rett syndrome patients, and thereby contribute to osteopenia.

Further, the connection between $\mathrm{MeCP} 2$ and autoimmune disease represents an interesting potential window to better understand how MeCP2 interacts with the immune system versus the nervous system in isolation. The functional comparison between MeCP2 alleles associated with autoimmune disease (Carmona et al., 2013; Cobb et al., 2010; Han et al., 2013; Kaufman et al., 2013; Koelsch et al., 2013; Sawalha et al., 2008; Webb et al., 2009), MeCP2 mutations resulting in Rett syndrome (Christodoulou et al., 2003), and Xq28 duplication in MeCP2 duplication syndrome (Ramocki et al., 2010) may lead to a better understanding of how different perturbations of MeCP2 affect cellular function. For instance, assessment of alleles associated with autoimmune disease may reveal preserved neuronal function, with impaired immune cell function, and indicate regions of the $\mathrm{MeCP} 2$ gene or protein that are more or less important to function in specific cell types. Such an approach could both advance our understanding of MeCP2 as a molecule, and also its role in human disease.

\section{Conclusion}

The role of MeCP2 in the immune system, like the nervous system, is complex, nuanced, and will likely take years of work before its many levels of immunologic regulation can be confidently explained. Here, it was demonstrated that $\mathrm{MeCP} 2$ is an important regulator of not only transcription within immune cells, but also that its overexpression leads to multi-point failures of response to infection, involving both immune and non- 
hematopoietic cell types. These results show that $\mathrm{MeCP} 2$ is an important regulator of immune function on multiple levels, and that in its absence or overexpression, proper control of the immune system is lost. Future work should endeavor to further understand both the molecular pathways influenced by $\mathrm{MeCP} 2$, and how these MeCP2-regulated pathways contribute to effective orchestration of the immune system.

This dissertation significantly contributes to a growing understanding that MeCP2 is not limited to its function in neurons. Due to the dominant neurologic symptoms of Rett syndrome (Chahrour and Zoghbi, 2007), research on MeCP2 has likewise been dominated by the study of neurons (Guy et al., 2011), with very little consideration given to any other cell types. Even in the first publications detailing mouse models of Mecp2 deletion, it was suggested that neuronal MeCP2 alone accounts for disease (Chen et al., 2001). However, subsequent publications demonstrated that MeCP2 is, in fact, expressed in not only other CNS cell types (Ballas et al., 2009; Cronk et al., 2015a; Maezawa and Jin, 2010; Nguyen et al., 2013), but also that re-expression of MeCP2 in non-neuronal cells leads to significant amelioration of disease (Cronk et al., 2015a; Lioy et al., 2011; Nguyen et al., 2013).

Prior to the work presented here, it was known that microglia express Mecp2 (Maezawa and Jin, 2010), and that replacement of the immune system with wild type cells, including engraftment of the CNS with peripheral-derived macrophages, improves outcomes in Mecp2-null mice (Derecki et al., 2012). However, the functional role of Mecp2 in macrophages and microglia was unknown. In addition, the physiologic effects of Mecp2 deletion on macrophages and microglia were unexplored. The data presented in 
this dissertation answered these questions by demonstrating that 1) Mecp2 regulates the transcriptional response to multiple stimuli in macrophages, and 2) Mecp2-null mice develop macrophage, microglia, and resident monocyte deficiency, in addition to inflammatory activation of microglia with disease progression (Cronk et al., 2015a). Unexpectedly, we also found that Mecp2 deficiency leads to neutrophilia in mice, which is driven by an overproduction of Gesf. This phenotype was likely related to our finding that Mecp2 represses expression of inflammatory gene products, as Mecp2-null macrophages overexpressed Gcsf upon inflammatory stimulation (Cronk et al., 2015a). These findings present some of the first evidence that MeCP2 loss-of-function may be directly associated with immunologic pathology. Tissue-resident macrophages are critical for tissue homeostasis and repair (Davies et al., 2013). Therefore, the role of MeCP2 in macrophage function may in fact represent a significant aspect of pathology in Rett syndrome, and even other immunologic disorders associated with MeCP2, such as SLE (Kaufman et al., 2013; Koelsch et al., 2013; Sawalha et al., 2008; Webb et al., 2009). The relative role of macrophages in these disorders of $\mathrm{MeCP} 2$ has yet to be fully understood. However, given the fact that essentially every tissue in the body contains a resident macrophage population (Davies et al., 2013), these cells may represent a previously unrecognized contributor to disorders of MeCP2 throughout the body.

In addition, the etiology of chronic, lethal respiratory infection in $\mathrm{MeCP} 2$ duplication syndrome had received little attention prior to this work. Previous groups had identified impaired IFN $\gamma$ production (Yang et al., 2012), and impaired antibody responses (Bauer et al., 2015), however a full investigation of respiratory infection in the context of 
$\mathrm{MeCP} 2$ overexpression had yet to be done. Here, we demonstrated that $\mathrm{MeCP} 2^{\mathrm{Tg} 3}$ mice are not only highly susceptible to influenza A infection, but we also identified multiple novel disease processes during the course of respiratory infection in the context of MeCP2 overexpression. These included neutrophilia, cytokine storm, excessive glucocorticoid release, defects of both $\mathrm{T}$ and $\mathrm{B}$ cell responses, and, importantly, nonhematopoietic pathology, including pulmonary hemorrhage. This knowledge may help to both explain, and eventually treat, respiratory infection in $\mathrm{MeCP} 2$ duplication patients. Although anti-inflammatory COX-2 inhibitor treatment did not rescue mortality in $\mathrm{MeCP} 2^{\mathrm{Tg} 3}$ mice, it was effective in ameliorating disease symptoms, and might be utilized in patients for symptomatic treatment, especially in the context of non-lethal infection. Future work should aim to recapitulate our findings of excessive innate inflammation during infection and explore the efficacy of anti-inflammatory treatments.

The cumulative results presented here demonstrate that $\mathrm{MeCP} 2$ is an essential molecule for proper immune function. If one summary statement could be made regarding this body of work, it would be that $\mathrm{MeCP} 2$ is critical for the control of response to immunologic stimuli. In a healthy environment, little outward immunologic dysfunction may be expected from MeCP2 mutant or overexpressing cells. However, once that cell is stimulated, its hidden malfunction is revealed. Further, it is likely that a combination of many malfunctions by multiple cell types lead to the full disease observed in both Rett and MeCP2 duplication syndromes. Although neurologic dysfunction is core to both disorders, the importance and relevance of the immune system, and indeed nonneuronal cells of many types, should be a major focus of future work. 


\section{Figures}

\section{Figure 14.}

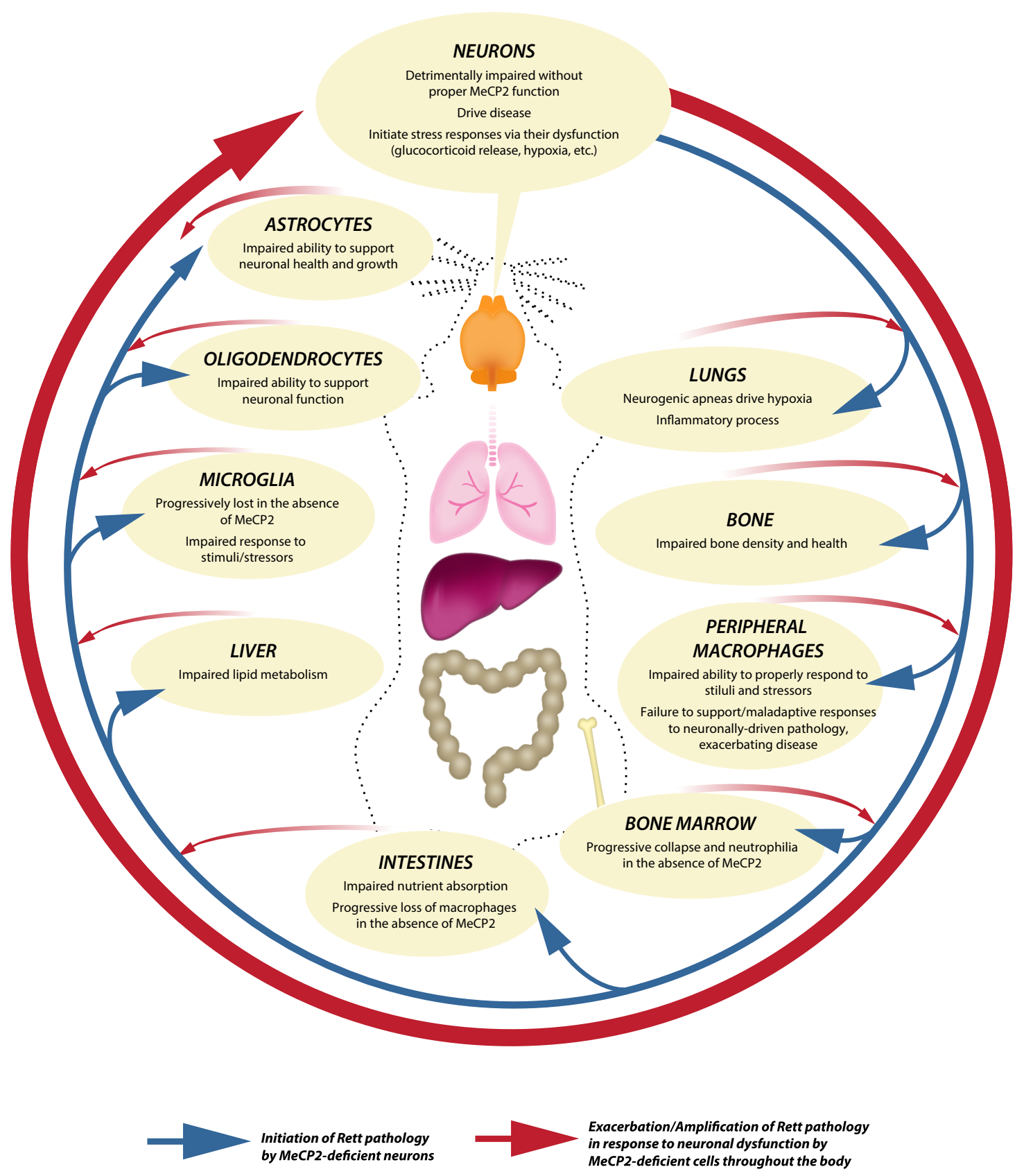

*Adapted from “Unexpected cellular players in Rett syndrome pathology," Cronk et al. 2015* 
Schematic of potential pathologic synergy between neuronal and non-neuronal cell types during disease progression of Mecp2-deficient mice. Neurons initiate and drive pathology in MeCP2-deficient mice, which is further amplified and exacerbated by aberrant responses from MeCP2-deficient cells throughout the body. $\mathrm{MeCP} 2$ is critically important for neuronal function, and in its absence, multiple neurologic impairments lead to downstream negative systemic events, such as glucocorticoid release and apneas (leading to hypoxia). MeCP2-deficient cells throughout the body, impaired in their function without $\mathrm{MeCP} 2$, in turn respond in a maladaptive fashion, either failing to support or actively exacerbating pathology. This results in amplification of disease by causing further neuronal dysfunction. In turn, further neuronal pathology results in exacerbation of peripheral dysfunction, ultimately resulting in a positive feedback loop, which drives perpetual amplification of the disease. Specific examples include the presence of inflammatory processes in the lung; impaired bone density; impaired response to stimuli and stressors and loss of peripheral macrophages; progressive bone marrow collapse and neutrophilia; impaired nutrient absorption in the intestines; impaired lipid metabolism in the liver; progressive loss and impaired response to stimuli and stressors by microglia; impaired ability of oligodendrocytes to support neuronal function; and impaired ability of astrocytes to support neuronal health and growth.

\section{Figure and legend adapted from:}

Cronk, J.C., Derecki, N.C., Litvak, V., and Kipnis, J. (2015b).

Unexpected cellular players in Rett syndrome pathology. Neurobiology of disease. doi: 10.1016/j.nbd.2015.05.005 
Figure 15.

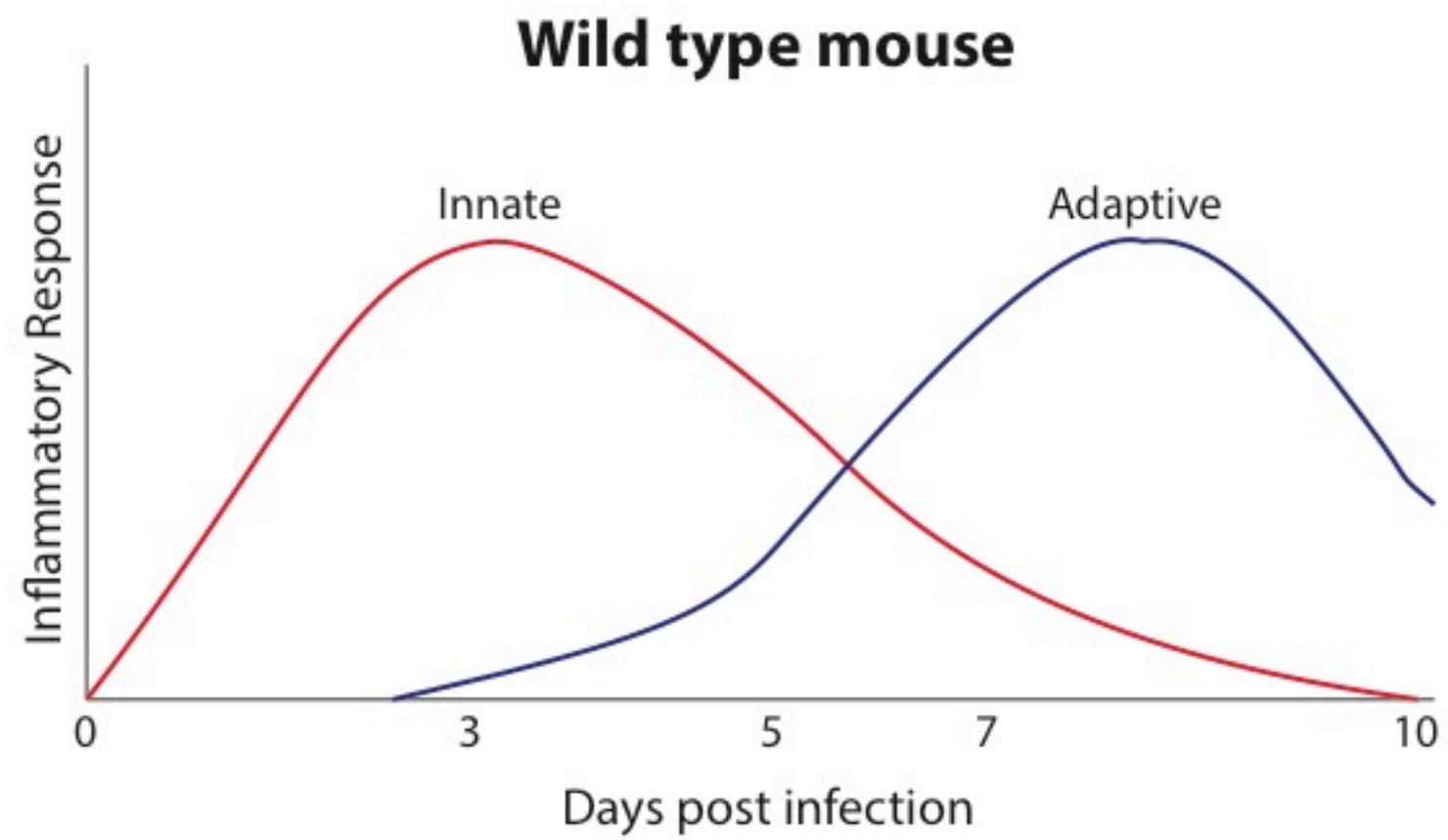

MeCP2 $2^{\text {Tg3 }}$ mouse

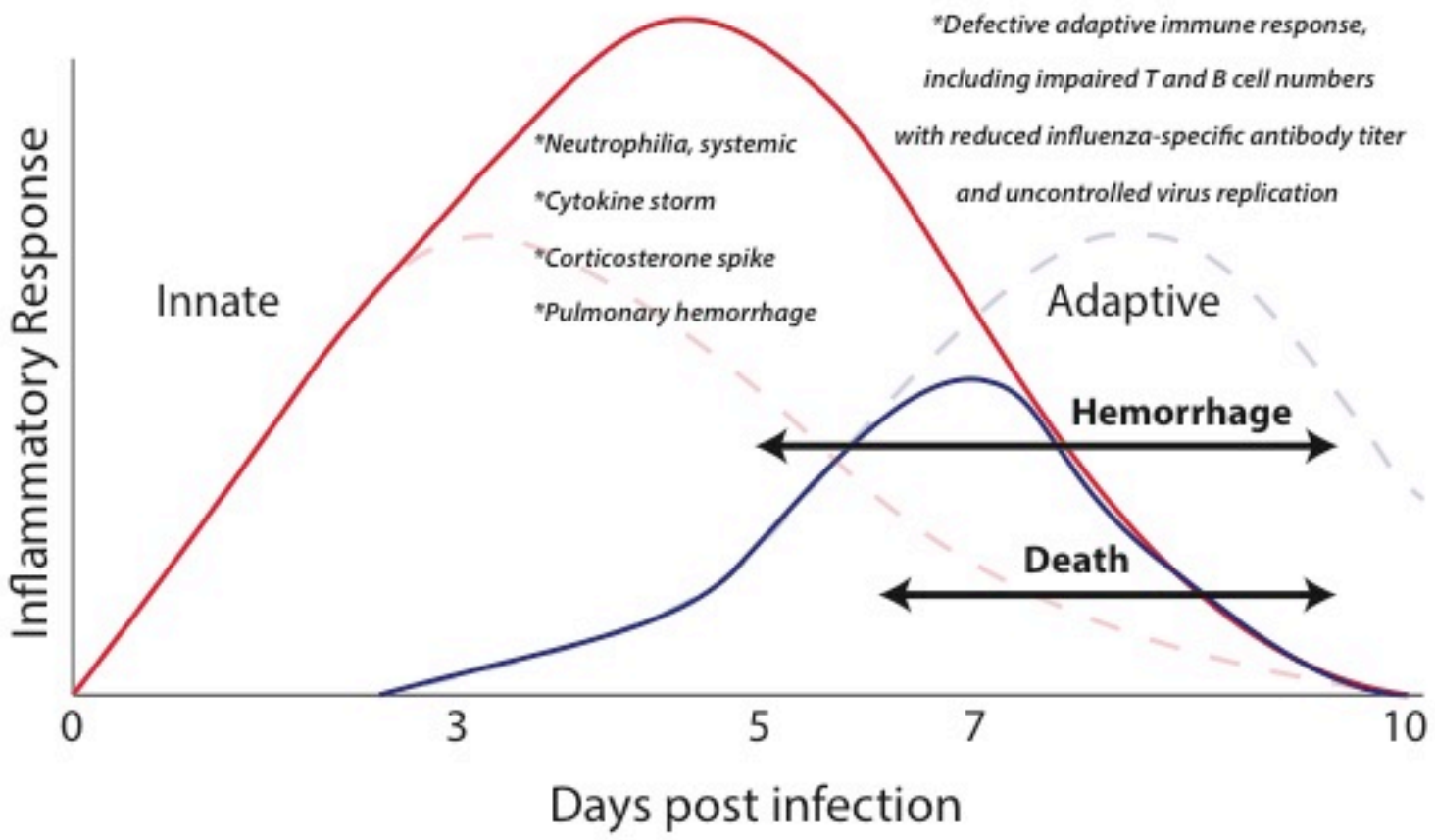


Schematic of pathologic events during influenza $\mathrm{A}$ infection in $\mathrm{MeCP2}^{\mathrm{Tg} 3}$ mice. Wild type mice have an innate immune response early during infection, which tapers off as the adaptive immune response arrives in the lungs approximately one week post-infection, with resolution of infection and inflammation underway by 10 days post-infection. In contrast, influenza A infection in $\mathrm{MeCP} 2^{\mathrm{Tg} 3}$ mice is characterized by excessive innate inflammation, increasing through day 5. This includes both peripheral and pulmonary neutrophilia, cytokine storm, excessive corticosterone levels, and pulmonary hemorrhage starting on day 5. By one-week post infection, when the adaptive immune response should undertake clearance and neutralization of virus and infected cells, $\mathrm{MeCP} 2^{\mathrm{Tg} 3}$ mice demonstrate defective adaptive immunity, including reduced $\mathrm{T}$ and $\mathrm{B}$ cell numbers with impaired influenza-specific antibody in the airways and uncontrolled virus replication. Ultimately, $\mathrm{MeCP} 2^{\mathrm{Tg} 3}$ mice succumb to infection between days 6-9 post-infection, with pulmonary hemorrhage as a probable cause of death. 


\section{Appendix}

Methyl-CpG binding protein 2 regulates microglia and

macrophage gene expression in response to inflammatory stimuli 


\section{Immunity}

\section{Methyl-CpG Binding Protein 2 Regulates Microglia and Macrophage Gene Expression in Response to Inflammatory Stimuli}

Graphical Abstract

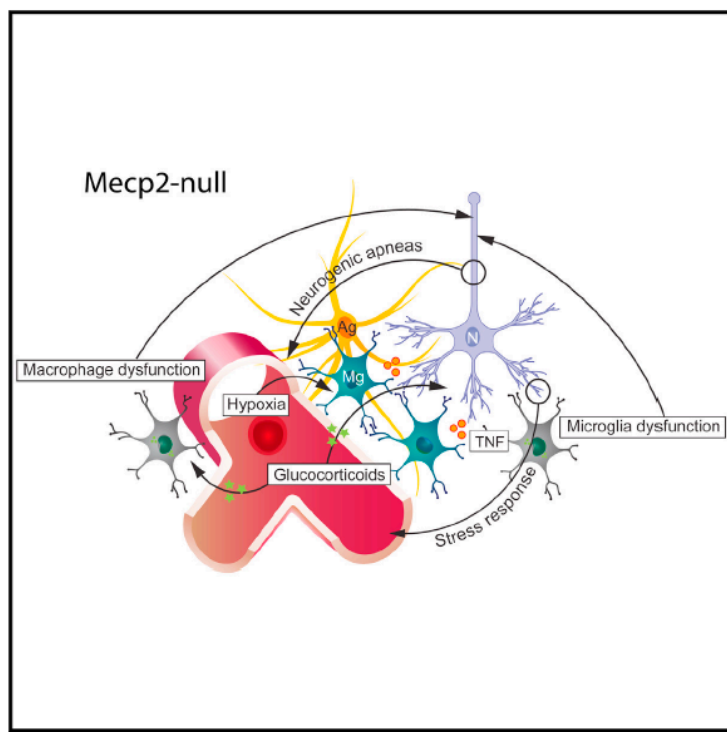

Highlights

- Mecp2 is broadly expressed in macrophages and resident monocytes

- Mecp2-null mice exhibit monocyte and resident macrophage deficiencies

- Mecp2 expression under $\mathrm{C} \times 3 \mathrm{cr} \mathbf{1}^{\text {creER }}$ extends survival of $M e c p 2^{\text {Lox-Stop/y }}$ mice

- Mecp2 regulates transcriptional responses to inflammatory stimuli

Cronk et al., 2015, Immunity 42, 679-691

CrossMark April 21, 2015 @2015 Elsevier Inc.

http://dx.doi.org/10.1016/j.immuni.2015.03.013
Authors

James C. Cronk, Noël C. Derecki, ...,

Vladimir Litvak, Jonathan Kipnis

Correspondence

vladimir.litvak@umassmed.edu (V.L.), kipnis@virginia.edu (J.K.)

In Brief

Mutations in the gene encoding MethylCpG binding protein 2 (MeCP2) are the primary cause of the neurodevelopmental disease Rett syndrome. Kipnis and colleagues demonstrate that Mecp2 regulates macrophage transcriptional responses to glucocorticoid and inflammatory stimuli, raising the possibility that abnormal macrophage responses contribute to systemic impairments in Rett syndrome.

Accession Numbers

GSE66211

GSE66501 
Appendix: Methyl-CpG binding protein 2 regulates microglia and macrophage

\section{Methyl-CpG Binding Protein 2 Regulates Microglia and Macrophage Gene Expression in Response to Inflammatory Stimuli}

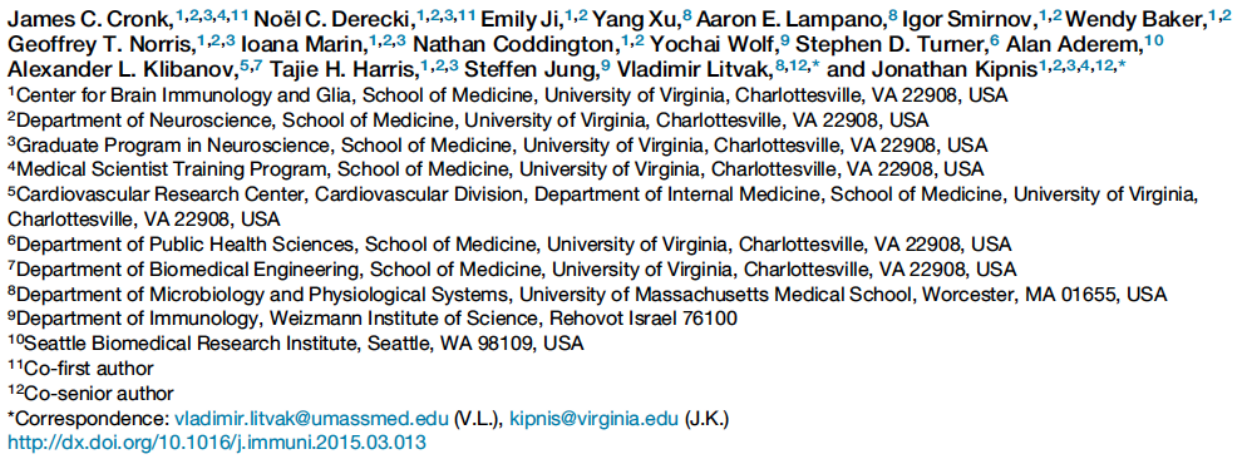

SUMMARY

Mutations in MECP2, encoding the epigenetic regulator methyl-CpG-binding protein 2, are the predominant cause of Rett syndrome, a disease characterized by both neurological symptoms and systemic abnormalities. Microglial dysfunction is thought to contribute to disease pathogenesis, and here we found microglia become activated and subsequently lost with disease progression in Mecp2null mice. Mecp2 was found to be expressed in peripheral macrophage and monocyte populations, several of which also became depleted in Mecp2null mice. RNA-seq revealed increased expression of glucocorticoid- and hypoxia-induced transcripts in Mecp2-deficient microglia and peritoneal macrophages. Furthermore, Mecp2 was found to regulate inflammatory gene transcription in response to TNF stimulation. Postnatal re-expression of Mecp2 using $C \times 3 c r 1^{\text {creER }}$ increased the lifespan of otherwise Mecp2-null mice. These data suggest that Mecp2 regulates microglia and macrophage responsiveness to environmental stimuli to promote homeostasis. Dysfunction of tissue-resident macrophages might contribute to the systemic pathologies observed in Rett syndrome.

\section{INTRODUCTION}

Rett syndrome, caused primarily by mutations in methyl-CpG binding protein 2 (MeCP2) (Amir et al., 1999), features prominent neurologic sequelae (Chahrour and Zoghbi, 2007); accordingly, efforts to understand the function of MeCP2 have been focused largely on its role in neurons (Chahrour and Zoghbi, 2007). Recent reports have found expression and roles for Mecp2 in astrocytes (Ballas et al., 2009; Lioy et al., 2011; Yasui et al., 2013), oligodendrocytes (Nguyen et al., 2013), and microglia (Derecki et al., 2012; Maezawa and Jin, 2010). In addition, Mecp2 is expressed in many tissues (Shahbazian et al., 2002). Thus, mutations in MeCP2 likely affect multiple organ systems and cell types, which is indeed reflected in the complexity of symptoms associated with Rett syndrome (Chahrour and Zoghbi, 2007; Dunn and MacLeod, 2001). While neurological symptoms are prominent, most girls with Rett syndrome also suffer from somatic impairments, including stunted growth, osteopenia, scoliosis, and digestive difficulties (Chahrour and Zoghbi, 2007; Dunn and MacLeod, 2001).

Many tissue-resident macrophages, including microglia, originate during embryonic hematopoiesis, beginning in the yolk sac and moving to the fetal liver. These precursor cells disseminate throughout tissues during embryogenesis, engraft within nearly every organ system, and form self-renewing populations (Ginhoux et al., 2010; Hashimoto et al., 2013; Kierdorf et al., 2013; Schulz et al., 2012; Yona et al., 2013). Other populations of tissue-resident macrophages, such as intestinal lamina propria intestinal macrophages, are constantly replenished by circulating monocytes (Bain et al., 2013; Varol et al., 2009). The functional roles of tissue-resident macrophages vary greatly and are dependent upon location and context (Davies et al., 2013). However, all tissue-resident macrophages appear to be unified by their role as provisioners of homeostatic maintenance (Davies et al., 2013). Further, monocyte-derived inflammatory non-resident macrophages are critical for effective response to infection and injury. In this context, these cells rely on a carefully balanced network of skewing paradigms, which direct macrophage

Immunity 42, 679-691, April 21, 2015 @2015 Elsevier Inc. 679 
Appendix: Methyl-CpG binding protein 2 regulates microglia and macrophage gene expression in response to inflammatory stimuli

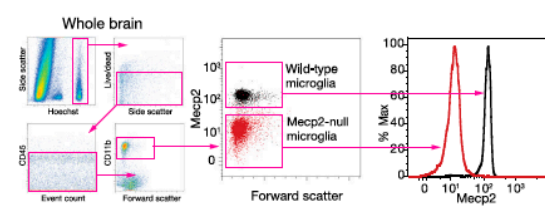

C
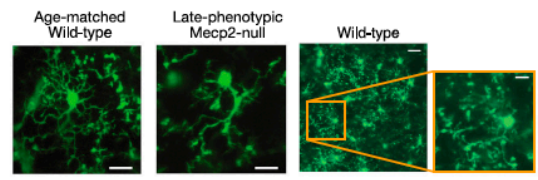

B

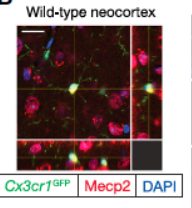

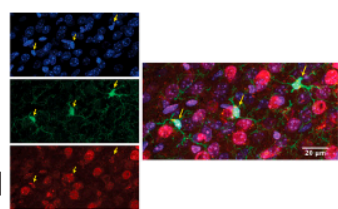
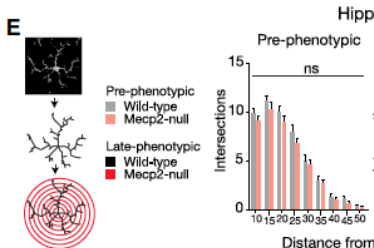

Hippocampus
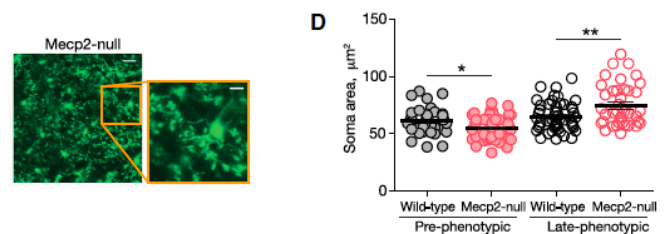

Cerebellum

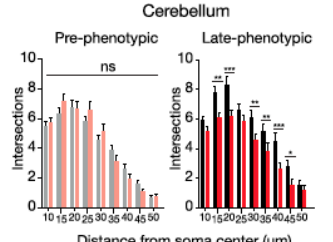

$\mathbf{F}$

G

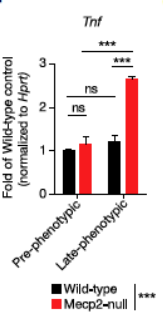

G
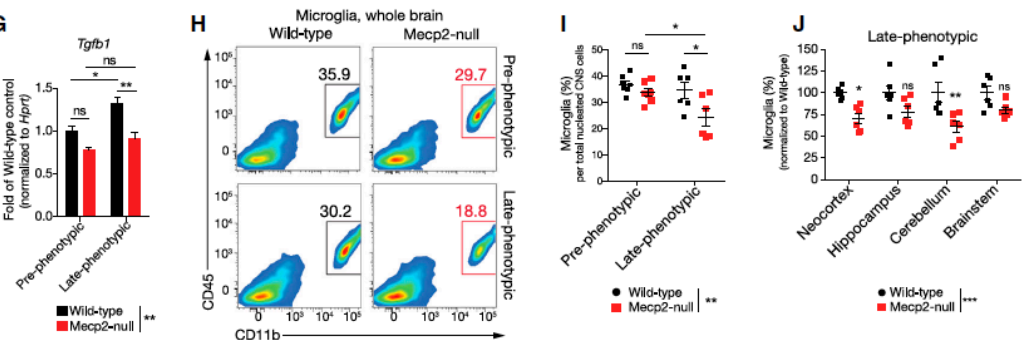

Figure 1. Microglia Become Activated and Subsequently Depleted with Disease Progression in Mecp2-Null Mice (A) Flow cytometry demonstrating Mecp2 expression in microglia from whole brain.

(B) Cryosections from $\mathrm{C} \times 3 \mathrm{Cr}^{\mathrm{GFP} /+}$ wild-type mice demonstrating Mecp2 expression in microglia (scale bar represents $20 \mu \mathrm{m}$ ). Images were cropped from larger images to allow for better visualization of Mecp2 localization within microglia.

(C) Representative stills from 2-photon live-imaging of late-phenotypic $C \times 3 \mathrm{cr} 1^{\mathrm{GFP} /+}$ wild-type and $\mathrm{C} \times 3 \mathrm{cr} 1^{\mathrm{GFP} /+} \mathrm{Mecp} 2$-null microglia.

(D) Quantitative assessment of microglial soma size measured by two-photon intravital microscopy in pre-and late-phenotypic $\mathrm{C} \times 3 \mathrm{cr} 1^{\mathrm{GFP} /+}$ wild-type and $C \times 3 c 7^{\mathrm{GFP} /+}$ Mecp2-null mice $\left(" \mathrm{p}<0.05\right.$; ${ }^{* *} \mathrm{p}<0.01$; two-way ANOVA with Bonferroni post-test; for pre-phenotypic $n=44$ wild-type and 54 Mecp2-null microglia; for late-phenotypic $n=51$ wild-type and $40 \mathrm{Mecp} 2$-null microglia. Microglia were measured from $n=3$ mice per group. Error bars represent SEM). (E) Sholl profiles for pre- and late-phenotypic wild-type and Mecp2-null microglia in hippocampus and neocortex showing intersections; ("*tp $p<0.001 ;$ **p $p<0.01$; (E) Sholl profiles for pre- and late-phenotypic wild-type and Mecp2-null microglia in hippocampus and neocortex showing intersections; ("*t" $p<0.001$; " $p<0.01$;
${ }^{*} p<0.05$; two-way ANOVA with Bonferroni post-test, $n=3$ mice per group with three separate areas from slices from identical brain structures analyzed per each $" \mathrm{p}<0.05$; two-way ANOVA with Bonferroni post-test, $n=3$ mice per group with three separate areas from slices from identical brain structures analyzed per each genotype. Data are presented as mean \pm SEM)

(F and G) qRT-PCR of Tnf (F) and Tgfb1 (G) from pre- and late-phenotypic Mecp2-null mice and their age-matched wild-type controls ("p $<<0.05$; "* $p<0.01$; *"* $p<$ 0.001; two-way ANOVA with Bonferroni post-test, $n=3$ mice for all groups except late-phenotypic wild-type for which $n=5$. Data are presented as mean \pm SEM). (H) Flow cytometric analysis demonstrating the percentage of microglia in whole brain preparations in Mecp2-null mice with increasing disease severity. Numbers show the percentage of Hoechst+ (nucleated) singlet CNS cells that are $\mathrm{CD} 45^{\mathrm{l}} \mathrm{CD} 11 \mathrm{~b}^{+}$microglia.

(I) Quantification of microglia in pre- and late- phenotypic Mecp2-null mice as compared to age-matched wild-type controls. Cells were gated on Hoechst ${ }^{+}$ (nucleated cells), Singlets, Size, and CD45 ${ }^{\text {Io/neg }}$ to exclude peripheral immune cells. (Two-way ANOVA with Bonferroni post-test; pre-phenotypic, not significant. Late-phenotypic; ${ }^{*} p<0.05$. Pre-phenotypic versus late-phenotypic Mecp2-null; ${ }^{*} p<0.05$. Overall main effect wild-type versus Mecp2-null; $*{ }^{*}<0.01 . n=7$ pairs

680 Immunity 42, 679-691, April 21, 2015 ๑2015 Elsevier Inc.

(legend continued on next page) 
Appendix: Methyl-CpG binding protein 2 regulates microglia and macrophage gene expression in response to inflammatory stimuli

function including the initiation and resolution of inflammation, clearance of debris and pathogens, and assistance in the healing process (Sica and Mantovani, 2012). Notably, mice lacking macrophage colony stimulating factor 1 receptor (CSF-1R) are deficient in all macrophages and are characterized by multiple organ failures and shortened lifespan (Dai et al., 2002), emphasizing the critical importance of macrophages in support of bodily tissues.

Our previous work demonstrated that engraftment of wild-type monocytes into the brains of Mecp2-null mice (through bonemarrow transplantation) extends lifespan by several months and improves neurologic and behavioral outcomes (Derecki et al., 2012). In addition, phagocytosis of apoptotic cells in vitro is impaired in Mecp2-null microglia. Although brain engraftment by monocytes with bone-marrow transplant is crucial for significant lifespan extension in Mecp2-null mice (Derecki et al., 2012), we did not explore the possibility that Mecp2 might be important for the normal function of other mononuclear phagocytes.

Here we demonstrated that numerous populations of macrophages and monocytes expressed Mecp2, and that Mecp2null mice become deficient in several macrophage populations, including microglia. We next showed that postnatal re-expression of Mecp2 under a $C \times 3 c r 1$-inducible promoter resulted in a lifespan increase in otherwise Mecp2-null mice. In order to elucidate mechanisms behind the macrophage defects in the context of Mecp2-deficiency, we demonstrated by RNA-Seq that acutely isolated Mecp2-null microglia and peritoneal macrophages displayed changes in specific signaling pathways, suggesting that Mecp2 is an important regulator of microglia and macrophage gene expression. Further in vivo and in vitro validation studies confirmed that Mecp2 is important for proper transcriptional regulation of multiple gene-expression programs in macrophages. Overall, these results demonstrated that Mecp2 is an important epigenetic regulator of macrophage response to stimuli and stressors.

\section{RESULTS}

Microglia Become Activated and Subsequently Depleted with Disease Progression in Mecp2-Null Mice Our previous data (Derecki et al., 2012) showing a role for microglia in disease pathogenesis of Mecp2-deficient mice led us to study in greater detail the role of Mecp2 in microglia and developmentally related peripheral tissue-resident macrophages. Wild-type microglia were found to express Mecp2, as examined by intracellular flow cytometric labeling (Figure 1A) or by in situ immunofluorescence (Figure 1B). This is in line with previously reported results (Maezawa and Jin, 2010).

We next investigated how loss of Mecp2 affects microglia in vivo. Using a thinned-skull technique, we performed intravital two-photon imaging on pre- and late-phenotypic Mecp2-null and wild-type mice. We observed that pre-phenotypic Mecp2null microglia had significantly smaller somas (similar to Mecp2-null neurons and astrocytes), whereas late-phenotypic
Mecp2-null microglia soma were larger in size as compared to wild-type (Figures 1C and 1D; Movies S1 and S2), suggestive of microglia activation (Kozlowski and Weimer, 2012). In addition, in situ Sholl analysis demonstrated that while pre-phenotypic Mecp2-null microglia were not different from wild-type, microglia from late-phenotypic mice displayed significantly reduced process complexity in three examined brain areas (hippocampus, neocortex, and cerebellum; Figure 1E). Together, the findings of increased soma size and decreased process complexity suggested that Mecp2-null microglia became activated with disease progression. Indeed, qRT-PCR of acutely isolated Mecp2-null microglia from pre- and late-phenotypic mice showed increased Tnf mRNA encoding the pro-inflammatory cytokine tumor necrosis factor (TNF) (Figure 1F) while Tgfb1 transcription, required for microglia maintenance (Butovsky et al., 2014), was decreased in late-phenotypic microglia (Figure 1G).

We next examined Mecp2-null microglia by flow cytometry, which revealed a progressive loss of microglia in Mecp2-null mice from pre- to late-phenotypic stage (Figures $1 \mathrm{H}$ and 1I). Loss of microglia was found throughout the brain (Figure 1J). The loss was also observed in brains of late-phenotypic Mecp2-null mice by immunohistochemistry (Figures S1A and S1B). Further, immunohistochemical staining for Iba1, a microglial marker, and for cleaved caspase 3 (CC3), a marker of apoptotic cells, revealed sporadic $\mathrm{CC}^{+}$microglia in late-phenotypic Mecp2-null brains (Figure S1C). In sum, these data suggest that in the context of Mecp2-deficiency, microglia become activated and are lost with disease progression.

Meningeal Macrophages Are Lost with Disease Progression in Mecp2-Null Mice

When analyzing microglia by intravital two-photonmicroscopy, we also observed significant morphologic disruption of $\mathrm{C} \times 3 \mathrm{cr} 1^{\mathrm{GFP} /+}$ meningeal macrophages in vivo in late-phenotypic Mecp2-null mice suggestive of reactive phenotype (Figure 2A). To assess meningeal macrophages in further detail, we performed immunofluorescent labeling of meninges from pre-, mid-, and late-phenotypic Mecp2-null mice using antibodies against CD163 and F4/80. A progressive loss of macrophages at the late-phenotypic state was evident in Mecp2-null mice (Figures 2B and 2C).

Perivascular macrophages in the meninges are $\mathrm{F} 4 / 80^{+}$ $\mathrm{CD}_{163^{+}}$, whereas a separate meningeal macrophage population is $\mathrm{F} 4 / 80^{+} \mathrm{CD} 163^{-}$(Davies et al., 2013). When we analyzed these populations separately, we found that perivascular macrophages $\left(\mathrm{F} 4 / 80^{+} \mathrm{CD} 163^{+}\right)$were progressively lost in Mecp2null mice (Figure 2D), whereas non-perivascular meningeal macrophages $\left(\mathrm{F} 4 / 80^{+} \mathrm{CD} 163\right)$ were not significantly depleted (Figure 2E).

Peripheral Monocytes and Macrophages Express Mecp2, and Some Are Lost in Mecp2-Null Mice

Given the phenotype of meningeal macrophages and microglia, we decided to expand our analysis to additional populations of peripheral macrophages. Many tissue-resident macrophages,

of wild-type and Mecp2-null mice pooled from 3 independent experiments for pre-phenotypic groups and n = 6 pairs of wild-type and Mecp2-null mice pooled from four independent experiments for late-phenotypic groups. Error bars represent SEM).

(J) Brain region-specific microglia percentages as measured by flow cytometry in late-phenotypic Mecp2-null mice and age-matched wild-type controls ("t $<<0.05 ;{ }^{* *} p<0.01 ; * * t p<0.001$; two-way ANOVA; $n=6$ mice per group. Error bars represent SEM). 
Appendix: Methyl-CpG binding protein 2 regulates microglia and macrophage gene expression in response to inflammatory stimuli

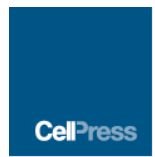

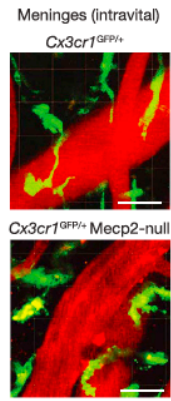

Wild-type

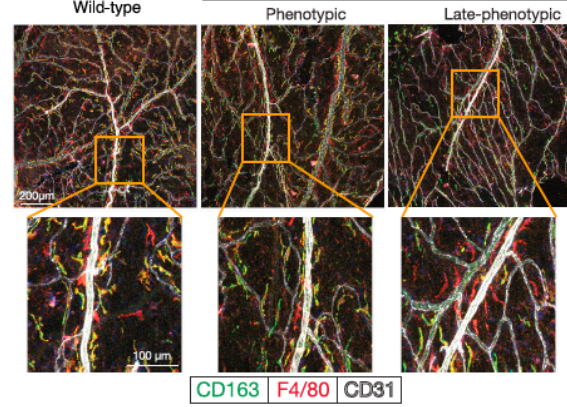

C

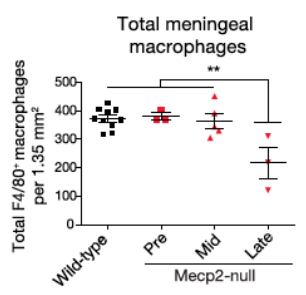

D

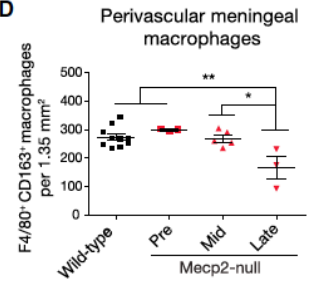

E

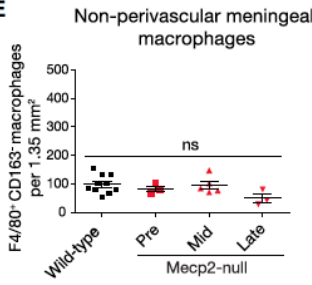

Figure 2. Meningeal Macrophages Are Lost with Disease Progression in Mecp2Null Mice

(A) Representative stills from intravital twophoton microscopy of phenotypic Mecp2-null meningealmacrophages demonstrating abnomal meningealmacrophages den

(B) Representative images of wild-type, mid and late-phenotypic Mecp2-null meningeal macrophages. Upper panels, scale bar represents $200 \mu \mathrm{m}$. Lower panels, scale bar represents $100 \mu \mathrm{m}$.

(C) Quantification of total meningeal macrophages in late-phenotypic Mecp2-null mice as compared to wild-type, pre- and mid-phenotypic Mecp2-null (one-way ANOVA with Bonferroni post-test; " ${ }^{*} \mathrm{p}<$ 0.01. Error bars represent SEM)

(D) Quantification of $\mathrm{F} 4 / 80^{+} \mathrm{CD} 163^{+}$perivascular (D) Quantification of $\mathrm{F} 4 / 80^{+} \mathrm{CD} 163^{+}$perivascular meningeal macrophages in late-phenotypic Mecp2-null mice as compared to wild-type, pre and mid-phenotypic Mecp2-null (one-way ANOVA with Bonferroni post-test; ${ }^{*} p<0.01 ;{ }^{* *} p<0.01$. Error bars represent SEM).

(E) Quantification of $\mathrm{F} 4 / 80^{+} \mathrm{CD} 163^{-}$non-perivascular meningeal macrophages in Mecp2-null mice as compared to wild-type (one-way ANOVA with Bonferroni post-test. Error bars represent SEM).

Because both $\mathrm{CD} 64^{+} \mathrm{F} 4 / 80^{+} \mathrm{CD} 11 \mathrm{~b}^{+}$intestinal macrophages and $\mathrm{Ly}_{6} \mathrm{c}^{\mathrm{lo}}$ monocytes share their derivation from Ly6c $\mathrm{c}^{\text {hi }}$ monocytes (Bain et al., 2013; Varol et al., 2009; Yona et al., 2013), it is possible that the reductions seen in these populations even in pre-phenotypic Mecp2-null mice might reflect their common origin. To this end, we tested whether Ly6c $\mathrm{c}^{\text {hi }}$ monocytes from bone marrow were impaired in their ability to differentiate and/or proliferate as macrophages in response to MCSF in vitro.

including microglia, originate from similar progenitor pools during embryogenesis (Ginhoux et al., 2010; Hashimoto et al., 2013; Kierdorf et al., 2013; Schulz et al., 2012; Yona et al., 2013), and might therefore share pathologies in the context of Mecp2-deficiency. We found that all tested tissue-resident macrophages expressed Mecp2 (Figures $3 \mathrm{~A}$ and $3 \mathrm{~B}$ ). In addition, Ly $6 \mathrm{c}^{\mathrm{lo}}$ monocytes, which represent a longer-lived resident population in the blood (Yona et al., 2013) also expressed Mecp2 (Figure 3B). In contrast, Ly6c ${ }^{\text {hi }} \mathrm{CCR}^{+}$monocytes and neutrophils expressed low or undetectable amounts of Mecp2 (Figure 3C).

As expected, based on the microglia and meningeal macrophage results described above, we found reductions of other peripheral macrophage populations in Mecp2-null mice. Unlike microglia, $\mathrm{CD}^{+} 4^{+} \mathrm{F} 4 / 80^{+} \mathrm{CD} 11 \mathrm{~b}^{+}$macrophages from the small intestine were reduced in both pre- and late-phenotypic Mecp2null mice (Figures $3 \mathrm{D}$ and $3 \mathrm{E}$ ). Circulating Ly $6 \mathrm{c}^{\mathrm{lo}}$ monocytes were also reduced in number in both pre- and late-phenotypic Mecp2-null mice (Figures 3F and 3G).

We found that Mecp2-null Ly6c ${ }^{\text {hi }}$ monocytes differentiated into macrophages with similar kinetics to wild-type and produced similar numbers of macrophages per monocyte, making it an unlikely possibility that Mecp2 is directly necessary for Ly $6 c^{\text {hi }}$ monocyte differentiation or macrophage proliferation in the absence of other factors (Figure S2).

Although Mecp2 did not affect differentiation or proliferation in vitro, we hypothesized that Mecp2 might play a role in monocyte/macrophage responses within the context of the full disease caused by whole-animal Mecp2-deficiency. To test this possibility, we depleted monocytes and macrophages using intravenous (i.v.) clodronate liposomes in wild-type and Mecp2null littermates (mid-phenotypic, 6 weeks to allow for immune system maturation but prior to the peak of disease) and measured the repopulation of resident monocytes. Dil liposomes were injected i.v. 2 days after clodronate liposome injection, similar to published protocols (Sunderkötter et al., 2004). Ly6c ${ }^{\text {hi }}$ monocytes released from the bone marrow become Dil labeled and can be tracked in their differentiation to Ly $6 \mathrm{c}^{\mathrm{lo}}$ monocytes.

682 Immunity 42, 679-691, April 21, 2015 ๑2015 Elsevier Inc. 
Appendix: Methyl-CpG binding protein 2 regulates microglia and macrophage gene expression in response to inflammatory stimuli

On day 5 post-clodronate injection (day 3 post $\mathrm{Dil}$ ), wild-type and Mecp2-null mice had, as expected, begun to reconstitute their circulating monocyte populations, with a non-significant trend toward fewer total monocytes $\left(\mathrm{CD} 115^{+} \mathrm{CD} 11 \mathrm{~b}^{+}\right)$in Mecp2-null mice (Figures $3 \mathrm{H}$ and $3 \mathrm{l}$ ). However, when total monocytes were examined, Mecp2-null mice had significantly fewer resident Ly6 $6 \mathrm{c}^{\mathrm{lo}}$ monocytes as compared to wild-type (Figure 3J). In addition, when only $\mathrm{Dil}^{+}$monocytes were examined (representing only the circulating monocytes present on day 2 postclodronate injection), $\mathrm{Dil}^{+}$monocytes in wild-type mice had almost completely differentiated into $L y 6 \mathrm{c}^{\mathrm{lo}}$ resident monocytes, whereas Mecp2-null mice displayed a deficit in Ly6c $c^{1 \circ}$ monocyte differentiation (Figure $3 \mathrm{~K}$ ). The fact that some populations were deficient in the pre-phenotypic state (resident monocytes and intestinal macrophages), but others were lost progressively (microglia and meningeal perivascular macrophages) led us to hypothesize that Mecp2 is likely playing a complex role in macrophage biology which is not limited to macrophage survival and/ or death. Of note, we did not observe any difference in F4/ $80^{+} \mathrm{CD}_{163^{-}}$meningeal macrophages (Figure $2 \mathrm{E}$ ) or splenic red pulp macrophages (data not shown), which also express Mecp2 (Figures $3 \mathrm{~A}$ and $3 \mathrm{~B}$ ), further suggesting that the role of Mecp2 in macrophages is complex or upstream of cell loss and/or death, because not all macrophage populations were equally affected by Mecp2-deficiency. In addition, our data regarding microglia suggested inflammatory activation (Figures 1C-1G), implicating Mecp2-deficiency in processes beyond simple loss of microglia/macrophages. Overall, the data suggested a complex role for Mecp2 in macrophages, consistent with its previously described role in other cell types as an epigenetic regulator, affecting a multitude of genes (Guy et al., 2011).

Postnatal Expression of Mecp2 via Cx3cr $1^{\text {CreER }}$ in Otherwise Mecp2-Deficient Mice Increases Lifespan Previously we showed that transplantation of Mecp2-null mice with wild-type bone marrow increases lifespan, and that engraftment of microglia-like cells into the brain is important for this effect (Derecki et al., 2012). More recently, Cx3cr1 ${ }^{\text {creER }}$ mice have become available (Goldmann et al., 2013; Yona et al., 2013), and microglia are among a subset of macrophages with high expression of CX3CR1 during adulthood (Jung et al., 2000). Thus the $C x 3 c r^{\text {creER }}$ mouse can be used to efficiently target microglia, in addition to other CX3CR1-expressing monocytes and macrophages. Therefore, we crossed $\mathrm{C} \times 3 \mathrm{Cr}^{\text {creen }}$ mice with $M e c p 2^{\text {Lox-stop }}$ mice and their offspring $\left(C \times 3 c r 1^{\text {creER/+ }}\right.$ $M e c p 2^{\text {Lox-stop/y }}$ ) were treated with tamoxifen ( $\sim 9$ weeks of age), when symptoms just started to appear. In line with our previous finding with bone-marrow transplantation (Derecki et al., 2012), the lifespan of tamoxifen-treated $C \times 3 c r 1^{\mathrm{creER} /+}$ $M e c p 2^{\text {Lox-stop/y }}$ mice was significantly extended (Figure 4A) and weight loss was reversed as compared to oil-treated controls (Figure 4B), supporting the importance of microglia and macrophages in the arrest of pathology. In order to test for specificity of Mecp2 expression after tamoxifen treatment, we placed Cx3cr $1^{\text {creER/ }}{ }^{+} M e c p 2^{\text {Lox-stop/y }}$ mice on a tamoxifen diet for 3 months to maximize expression in any cells that would have the potential to recombine. As expected, we found no significant expression of Mecp2 in T or B cells, and partial re-expression in monocytes (Figure $4 \mathrm{C}$ ), as previously reported (Yona et al.,
2013). In the spleen, we found a small percentage of Mecp2-expressing red pulp macrophages ( 20\%) (Figure 4D), consistent with the fact that red pulp macrophages do not express high amounts of CX3CR1. However, $\mathrm{CD} 64^{+} \mathrm{F} 4 / 80^{+}$intestinal macrophages and microglia, in which CX3CR1 is highly expressed, had nearly $100 \%$ Mecp2 recombination (Figures $4 \mathrm{E}$ and $4 \mathrm{~F}$ ). Non-microglia cells in the CNS displayed no Mecp2 recombination (Figure 4G). Together, these results support that Mecp2deficiency in microglia/macrophages contributes to pathology and that restoring Mecp2-mediated regulation of transcriptional responses has the potential to mediate benefits in the context of whole-body Mecp2-deficiency.

Mecp2 Regulates Glucocorticoid and Hypoxia Responses in Microglia and Peritoneal Macrophages In order to define the functional role of Mecp2 in macrophages, we examined the global gene-expression profile in microglia and peritoneal macrophages derived from Mecp2-null mice. We detected increased expression of glucocorticoid-induced transcriptional signature genes in Mecp2-null cells when compared to their wild-type counterparts, suggesting that Mecp2 functions as a repressor of this pathway (Figures 5A-5D and Tables S1, S2, and S3). Among the dysregulated genes in Mecp2-null microglia and peritoneal macrophages, Fkbp5, a canonical glucocorticoid target gene, was strongly upregulated (Figures $5 \mathrm{~A}$ and $5 \mathrm{C}$ ). A number of studies have demonstrated that Mecp2 directly represses the Fkbp5 gene (Nuber et al., 2005; Urdinguio et al., 2008). Using chromatin immunoprecipitation (ChIP) analysis, we demonstrated Mecp2 binding to the Fkbp5 gene promoter in bone marrow-derived macrophages (BMDM) (Figure 5E). Furthermore, Mecp2 deletion resulted in increased amounts of histone $\mathrm{H} 4$ acetylation at cis-regulatory regions of Fkbp5 gene under basal conditions (Figure 5F). These results suggest that Mecp2 restrains Fkbp5 gene expression through epigenetic mechanisms. ChIP analysis demonstrated the binding of nuclear receptor co-repressor 2 (Ncor2) and histone deacetylase 3 (Hdac3) to the promoter region at the Fkbp5 gene (Figure S3). These results are consistent with the well-established role of Mecp2 in the recruitment of the Ncor2 and Hdac3 complex to target genes (Ebert et al., 2013; Lyst et al., 2013). We next examined the Fkbp5 gene-expression profile in dexamethasone-treated wild-type and Mecp2-null macrophages. Our results revealed that Mecp2 controls the sensitivity of the $F k b p 5$ gene to glucocorticoid stimulation. Mecp2 deletion resulted in the upregulation of $F k b p 5$ gene expression under dexamethasone stimulation at a low dose nomally incapable of triggering Fkbp5 gene expression (Figure $5 \mathrm{G}$ ).

We noticed that the glucocorticoid-induced gene set contains a number of hypoxia-inducible genes (Table S3). Furthermore, our gene set enrichment analysis demonstrated significantly increased expression of a subset of hypoxia-inducible genes in Mecp2-null microglia (Figures $5 \mathrm{H}$ and $5 \mathrm{I}$ ). To validate the cellintrinsic role for Mecp2 in the negative regulation of these hypoxia-inducible genes, we cultured BMDM under either normoxia or hypoxia conditions $\left(1 \% \mathrm{O}_{2}\right)$ and examined their mRNA using quantitative PCR analysis. We validated increased expression of three canonical hypoxia-inducible genes, Hif3a, Ddit4, and Cyr61 in Mecp2-null macrophages cultured under hypoxia conditions when compared to their wild-type 
Appendix: Methyl-CpG binding protein 2 regulates microglia and macrophage gene expression in response to inflammatory stimuli

CellPress

A

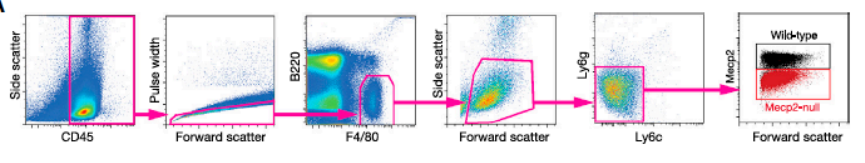

B

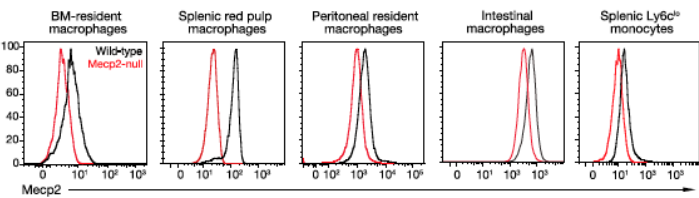

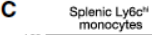

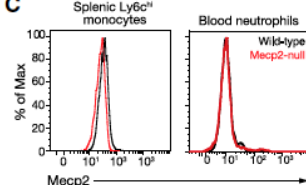

D
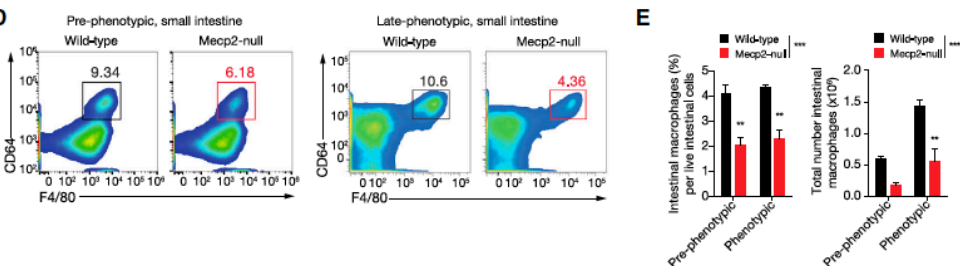

$\mathbf{F}$

Singlets, Live CD45+CD11b'Ly6g-CD115-SSC

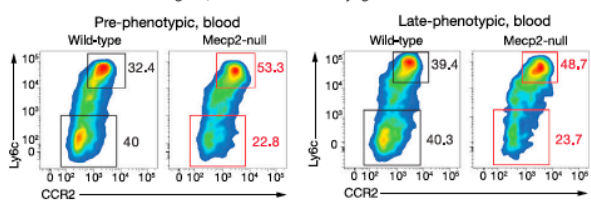

G
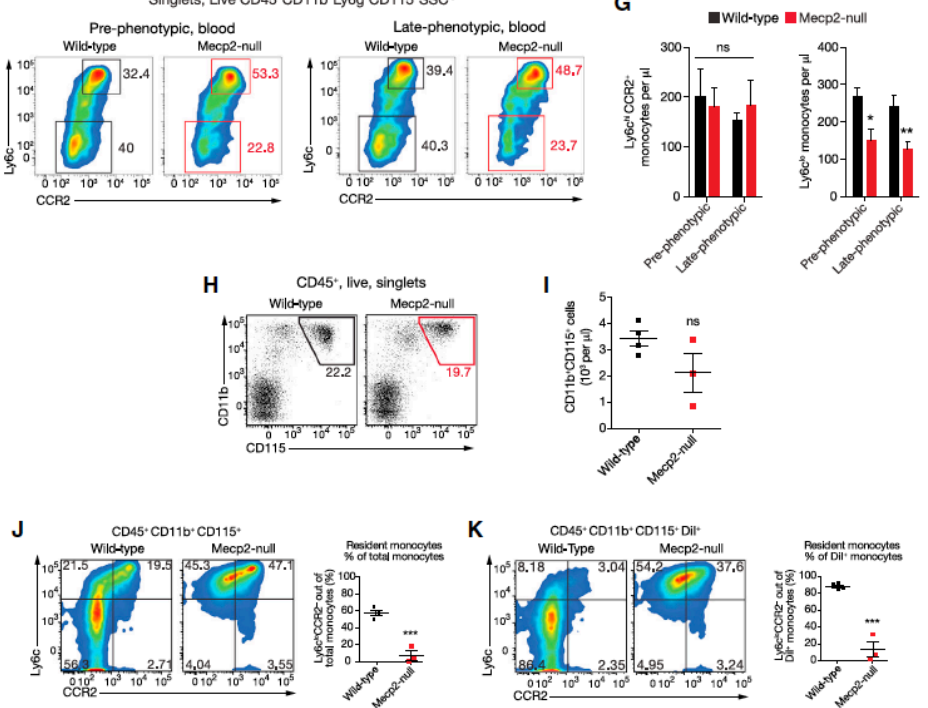

Figure 3. Peripheral Monocytes and Macrophages Express Mecp2, and Some Are Lost in Mecp2-Null Mice (A) Example gating strategy for Mecp2 expression; shown are red pulp macrophages from spleen.

(B) Mecp2 expression in macrophage and monocyte populations as measured by intracellular flow cytometric staining in wild-type and Mecp2-null mice. Gating:

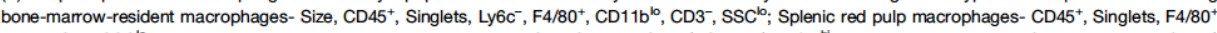

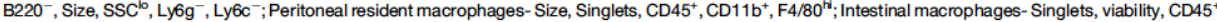

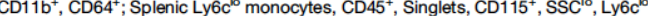

(legend continued on next page)

684 Immunity 42, 679-691, April 21, 2015 ๑2015 Elsevier Inc. 
Appendix: Methyl-CpG binding protein 2 regulates microglia and macrophage gene expression in response to inflammatory stimuli

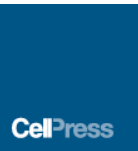

A

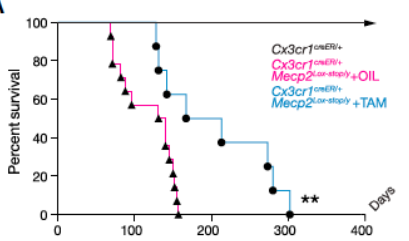

B

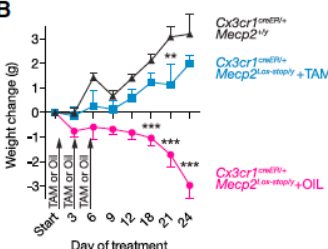

C
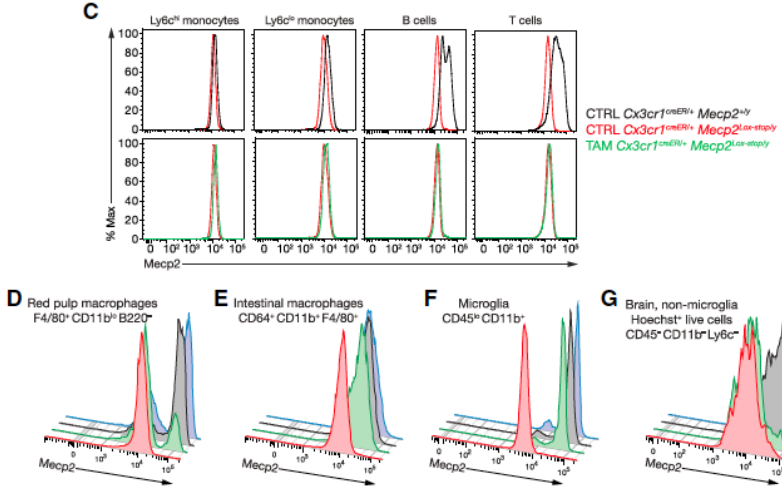

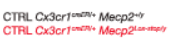

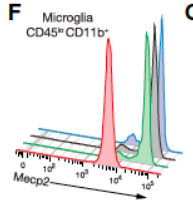

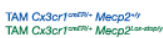

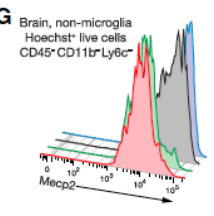

Figure 4. Postnatal Expression of Mecp2 via $\mathrm{C} \times 3 \mathrm{Cr}^{\mathrm{CreER}}$ in Otherwise Mecp2-Deficient Mice Increases Lifespan (A) Survival of $\mathrm{C} \times 3 \mathrm{Cr} 1^{\mathrm{CreER} /+} \mathrm{Mecp} 2^{\text {Lox-stop/y }}$ mice efter postnatal tamoxifen or oll control treatment (in phenotypic mice cox Cox test; tamoxifen treated $n=8$, oil treated $n=14)$.

(B) Weight change after postnatal tamoxifen or oil control treatment in phenotypic $\mathrm{C} x 3 \mathrm{Cr}^{\mathrm{CreER} /+}$ Mecp $2^{\text {Lox-stop/y }}$ mice ("tp $<0.01 ;$ "*tp $<0.001$. Two-way ANOVA with Bonferroni post-test; $n=5$ oil and $\mathrm{n}=3$ tamoxifen treated $\mathrm{CX} 3 \mathrm{Cr}^{\mathrm{creEF} /+}$ Mecp $2^{\text {Lox-stop/y. }} n=7 \quad C \times 3 c r 1^{\text {creen/ } /} M e c p 2^{\text {t/y }}$ tamoxifen treated. Astensks over Cxscri Mecp $2^{\text {Lx }}$-stopy oil-treated indicate comparisons

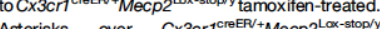
Asterisks over Cxscr1 Mecp2 2ax-stop/y tamoxifen-treated indicate companisons to

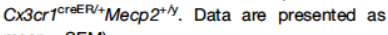
mean $\pm \mathrm{SEM}$ ).

(C-G) Analysis of Mecp2 re-expression in tamoxifen treated mice. Mice were fed tamoxifen food for 3 months to maximize possible recombination and analyzed by flow cytometry. Flow cytometry plots displaying Mecp2 expression in

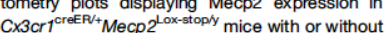
Mecp 2 ${ }^{2}$ - mice with or without $B$ and T rells; (D) red pulp macting monocytes, B, and T cell; (D) red pulp macrophages; (E) intestinal macrophages; ( $F$ ) microglia; and (G) total non-microglia nucleated cells in the CNS.

counterparts (Figure $5 \mathrm{~J}$ ), confirming a role for Mecp2 in regulation of at least a subset of hypoxia-induced gene transcripts.

Mecp2 Restrains Inflammatory Responses in Macrophages

Our RNA-seq analysis revealed increased expression of Tnf induced transcriptional signature genes in Mecp2-null microglia cells when compared to their wild-type counterparts (Table S2). These results indicate that Mecp2-deficiency leads to dysregu- lation of inflammatory responses in microglia and macrophages. To validate this finding, we examined the role of Mecp2 in the regulation of TNF-induced inflammatory responses in macrophages. We observed increased expression of $116, \mathrm{Tnf}, \mathrm{Cxc} / 2$, $\mathrm{CxCl} 3$, and Csf3 genes in TNF-stimulated Mecp2-null BMDM when compared to wild-type counterparts (Figure 6A). In order to corroborate these findings in vivo, we examined the inflammatory response of resident peritoneal macrophages. Mice were injected intraperitoneally with TNF, allowed to respond for $6 \mathrm{hr}$,

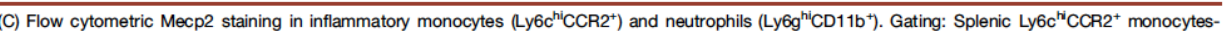

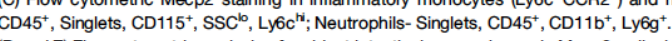

(D and E) Flow cytometric analysis of resident intestinal macrophages in Mecp2-null mice as compared to wild-type control. (D) Representative flow cytometry plots showing the percentage of $\mathrm{CD} 64^{+} \mathrm{F} 4 / 80^{+}$out of all $\mathrm{CD} 45^{+}$intestinal cells in pre- and late-phenotypic Mecp2-null mice as compared to age-matched wildtype controls. (E) Quantification of $\mathrm{CD} 64^{+} \mathrm{CD} 11 \mathrm{~b}^{+}$intestinal macrophages as measured by both percentage of total live intestinal cells (two-way ANOVA with Bonferroni post-test; " $p<0.01$; " ${ }^{* * t} p<0.001$ ) and absolute cell counts per animal (two-way ANOVA with Bonferroni post-test; " $p<0.01$; " ${ }^{* t *} p<0.001$ ). Data is representative of two independent experiments for phenotypic mice. Data are presented as mean \pm SEM.

(F) Flow cytometry plots of Ly6c ${ }^{\mathrm{h}} \mathrm{CCR} 2^{+}$and $\mathrm{Ly}_{6} \mathrm{c}^{\mathrm{lo}}$ blood monocytes in pre-and late-phenotypic Mecp2-null mice as compared to age-matched wild-type controls. Numbers represent percentage of cells out of Singlets, Live, CD $45^{+} \mathrm{CD} 11 \mathrm{~b}^{+} \mathrm{Ly} 6 \mathrm{~g}^{-} \mathrm{CD} 115^{+} \mathrm{SSC}^{\mathrm{lo}}$ monocytes.

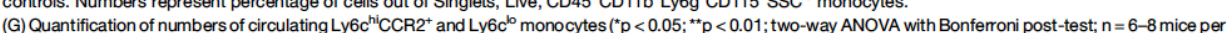
(G) Quantification of numbers of cir

group. Error bars represent SEM).
(H) Representative plots of $\mathrm{CD} 11 \mathrm{~b}^{+} \mathrm{CD} 115^{+}$total monocytes from blood of wild-type and Mecp2-null mice on day 5 post clodronate liposome injection.

(I) Monocyte count from peripheral blood of Mecp2-null and wild-type controls on day 5 post clodronate liposome injection (unpaired two-tailed Student's t test, not significant. Error bars represent SEM)

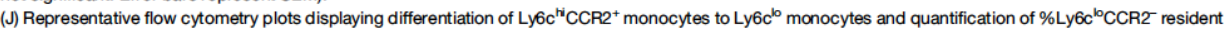
monocytes on day 5 post clodronate liposome injection; representative of two independent experiments. ("t"p $p<0.001$, unpaired two-tailed Student's t test. Error bars represent SEM).

(K) Same as $(\mathrm{J})$, except only $\mathrm{Dil}^{+}$monocytes are shown (cells labeled on day 2 post clodronate injection via Dil liposome injection); representative of two independent experiments. "*tp $<0.001$ (unpaired two-tailed Student's $t$ test. Error bars represent SEM). 
Appendix: Methyl-CpG binding protein 2 regulates microglia and macrophage gene expression in response to inflammatory stimuli

A

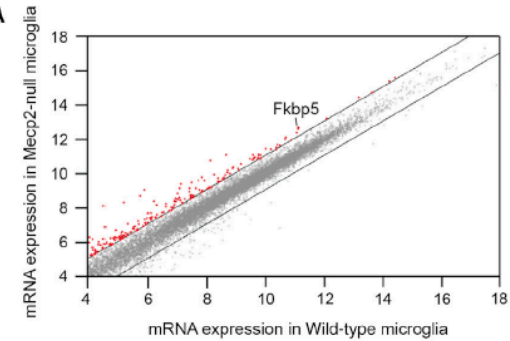

C
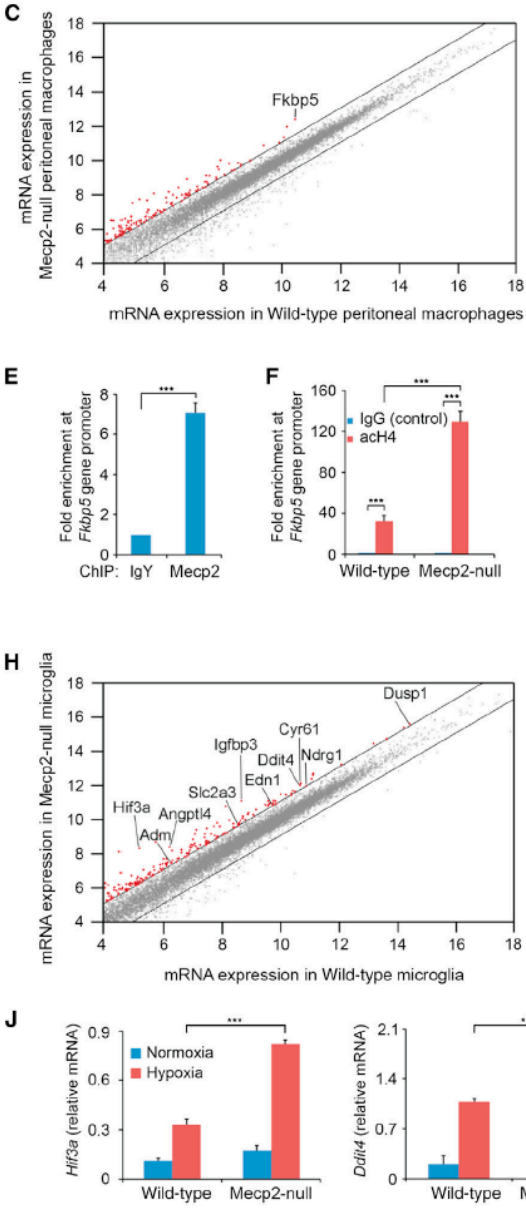

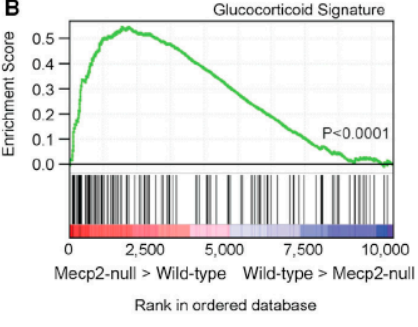

D

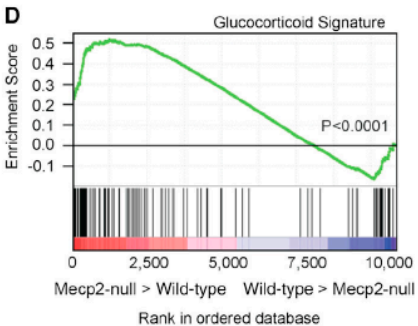

G
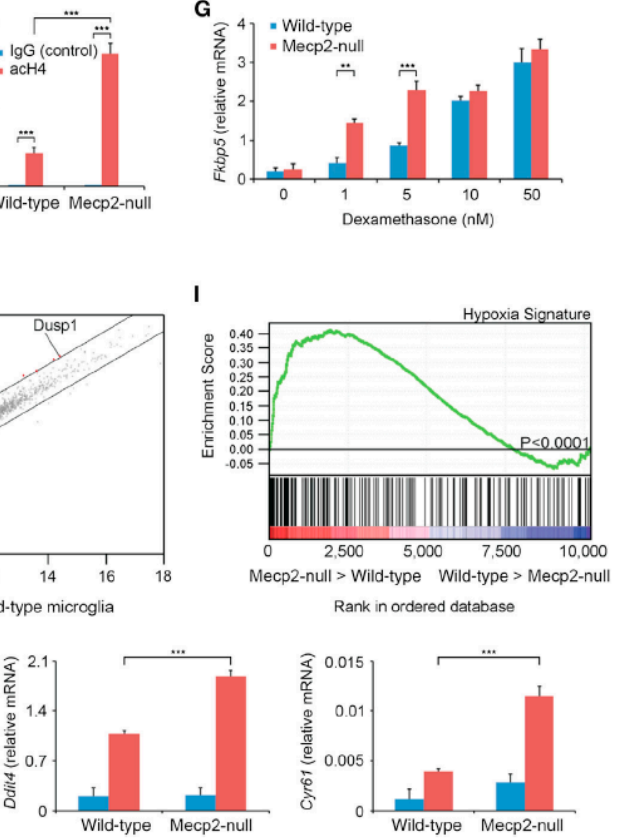

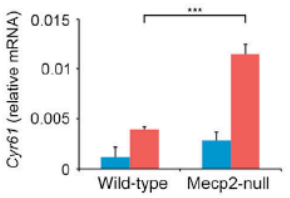

686 Immunity 42, 679-691, April 21, 2015 @2015 Elsevier Inc. 
Appendix: Methyl-CpG binding protein 2 regulates microglia and macrophage gene expression in response to inflammatory stimuli

and then peritoneal cells were collected by lavage. Cells from peritoneal lavage were positively selected for $\mathrm{F} 4 / 80^{+}$macrophages via AutoMACS. Peritoneal macrophages from Mecp2null mice injected with TNF displayed an altered transcriptional response as compared to wild-type (Figure 6B), including both over- and under-expression of multiple TNF response genes. No differences in baseline expression were evident in peritoneal macrophages from mice after saline treatment (Figure S4). Targets observed in vitro with TNF stimulated BMDMs were also overexpressed in peritoneal macrophages stimulated with TNF in vivo, suggesting that Mecp2 has consistent transcriptional roles across macrophage populations (Figures $6 \mathrm{~A}$ and $6 \mathrm{~B}$ ).

Csf3, the gene encoding granulocyte-colony stimulating factor (GCSF), was overexpressed in TNF-stimulated Mecp2-null macrophages both in vitro and in vivo (Figures $6 \mathrm{~A}$ and $6 \mathrm{~B}$ ). Late-phenotypic Mecp2-null mice without any manipulation also displayed increased GCSF protein in serum (Figure 6C). Because a well-established effect of GCSF is to stimulate neutrophil production (Bendall and Bradstock, 2014), we examined neutrophil numbers in Mecp2-null mice. Indeed, we found that Mecp2-null mice develop severe neutrophilia (Figures S5A and S5B). In addition, GCSF is known to drive the egress of hematopoietic stem cells (HSC) from the bone marrow (Bendall and Bradstock, 2014) and as expected, late-phenotypic Mecp2-null mice also became progressively deficient in bone marrow HSC with disease progression (Figures S5C and S5D). In order to test whether or not neutrophilia and HSC loss play a role in the disease, we treated Mecp2-null mice with a neutralizing antiGCSF antibody beginning at age 6-7 weeks (a mid-phenotypic time-point). Flow cytometric analysis revealed rescue of neutrophilia (Figure $6 D$ ) and prevention of HSC loss (Figure S5E). The treatment also moderately increased the lifespan of Mecp2-null mice (Figure 6E).

Together, these findings implicate Mecp2 in control of the macrophage inflammatory response, which might have down- stream implications for complex disease processes in the context of Mecp2-deficiency.

\section{DISCUSSION}

In this work, we have demonstrated that Mecp2 is widely expressed across macrophage populations, and that Mecp2deficiency leads to transcriptional impairment and/or loss of macrophages in multiple tissues. Postnatal genetic rescue of several tissue-resident macrophage and monocyte populations via $\mathrm{C} \times 3 \mathrm{cr}^{1^{\text {creer }}}$-mediated expression of Mecp2 resulted in increased lifespan. We have further provided evidence that Mecp2 is required for macrophage responses to multiple stimuli through both RNA-Seq of acutely isolated Mecp2-null microglia and peritoneal macrophages, and both in vitro and in vivo validation of pathways identified through RNA-seq. Together, these findings implicate macrophages, and potentially monocytes, as previously unrecognized players in Rett syndrome pathology. Our results also suggest that $\mathrm{Mecp} 2$ is an important regulator of macrophage response to stimuli/stressors, and future work examining the mechanisms of transcriptional and epigenetic regulation of macrophage responses should take into account the role of Mecp2 in macrophage activation.

These findings support the notion that both malfunction and loss of macrophages and resident monocytes might be a contributing factor in Rett syndrome pathologies occurring across multiple organ systems. Our finding that Mecp2-null macrophages display impaired response to multiple stimuliincluding glucocorticoids, hypoxia, and inflammation-might be of particular importance in the context of Mecp2-deficient animals, as the level of general organ dysfunction is high compared to wild-type mice; therefore, ongoing dysfunction of other cell types, such as neurons, might increase the cellular stress and/or activation upon Mecp2-null microglia and macrophages and thereby exacerbate their loss and/or further pathology

Figure 5. Mecp2 Regulates Glucocorticoid and Hypoxia Responses in Microglia and Peritoneal Macrophages

(A) Scatterplot comparing global gene-expression profiles between wild-type and Mecp2-null microglia. The black lines indicate a 2-fold cut-off for the difference in gene-expression levels. Data represent the average of six wild-type and three Mecp2-null samples, with each sample representing three pooled mice (thus, 18 mice and 9 mice respectively). mRNA expression is shown on a log2 scale.

(B) GSEA reveals the over-representation of glucocorticoid transcription signature genes in Mecp2-null microglia. The middle part of the plot shows the distribution of the genes in the glucocorticoid transcription signature gene set ("Hits") against the ranked list of genes. Data represent the average of six wild-type tribution of the genes in the glucocorticoid transcription signature gene set ("Hits") against the ranked list of genes. Data represent the average of six wild-type
and three Mecp2-null samples.

(C) Scatterplot comparing global gene-expression profiles between wild-type and Mecp2-null peritoneal macrophages as in (A). Data represent the average of six pooled wild-type and six pooled Mecp2-null mice.

(D) Global gene expression in wild-type and Mecp2-null peritoneal macrophages was analyzed as in (B). Data represent the average of six pooled wild-type and six pooled Mecp2-null mice.

(E) ChIP of Mecp2 from unstimulated wild-type macrophages showing binding of Mecp2 to the promoter region of the Fkbp5 gene. Data were normalized to lgY (negative control). Data represent the average of three independent experiments. ${ }^{* t *} p<0.001$ and ${ }^{* *} p<0.01$, (unpaired two-tailed Student's $t$ test. Data are presented as mean + SEM

(F) ChlP analysis of histone $\mathrm{H} 4$ acetylation at the Fkbp5 gene promoter in wild-type and Mecp2-null macrophages. Data represent the average of three independent experiments ("**"p $<0.001$ and ${ }^{* *} \mathrm{p}<0.01$; unpaired two-tailed Student'st test. Data are presented as mean \pm SEM).

(G) Dexamethasone-stimulation of wild-type and Mecp2-null macrophages was associated with a significant increase in Fkbp5 mRNA levels. Dexamethasone

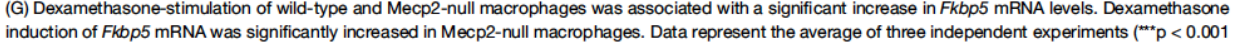
induction of Fkbp5 mRNA was significantly increased in Mecp2-null macrophages. Data reprees
and ${ }^{* t} \mathrm{p}<0.01$; unpaired two-tailed Student's t test. Data are presented as mean \pm SEM).

and ${ }^{* *} \mathrm{p}<0.01$; unpaired two-tailed Student's $\mathrm{t}$ test. Data are presented as mean $\pm \mathrm{SEM}$ ).
(H) Scatterplot comparing global gene-expression profiles between wild-type and Mecp2-null microglia. The black lines indicate a 2-fold cut-off for the difference (H) Scatterplot comparing global gene-expression profiles between wild-type and Mecp2-null microglia. The black lines indicate a 2-fold cut-off for the difference
in gene-expression levels. Data represent the average of six wild-type and three Mecp2-null samples, with each sample representing three pooled mice (18 and 9 mice respectively). mRNA expression levels are on a log2 scale.

(I) GSEA reveals the over-representation of hypoxia signature genes in unstimulated Mecp2-null microglia

(J) Wild-type and Mecp2-null macrophages were grown in hypoxia or normoxia for $24 \mathrm{hr}$. mRNA levels of Hif3a, Ddit4, and Cyr61 were measured using qRT-PCR. Results are representative of three independent experiments (average of three valuesc \pm CSEM; ${ }^{t \prime *} p<0.001$ by unpaired two-tailed Student's $t$ test). 
Appendix: Methyl-CpG binding protein 2 regulates microglia and macrophage gene expression in response to inflammatory stimuli
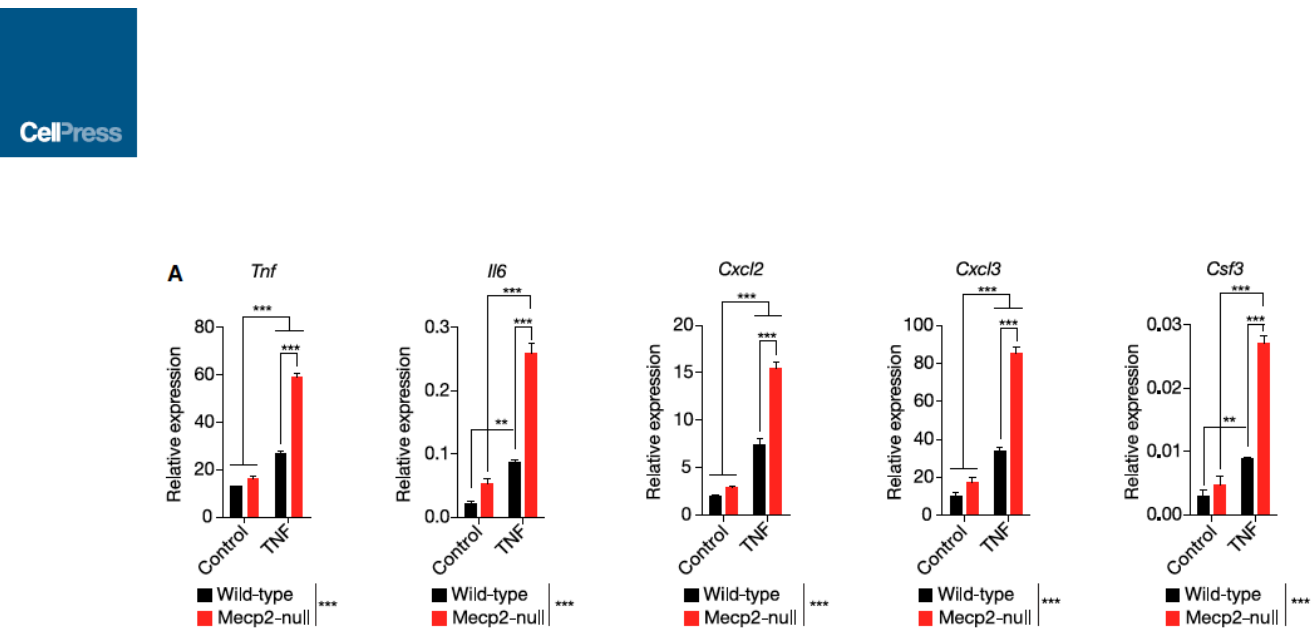

$\mathrm{B}$
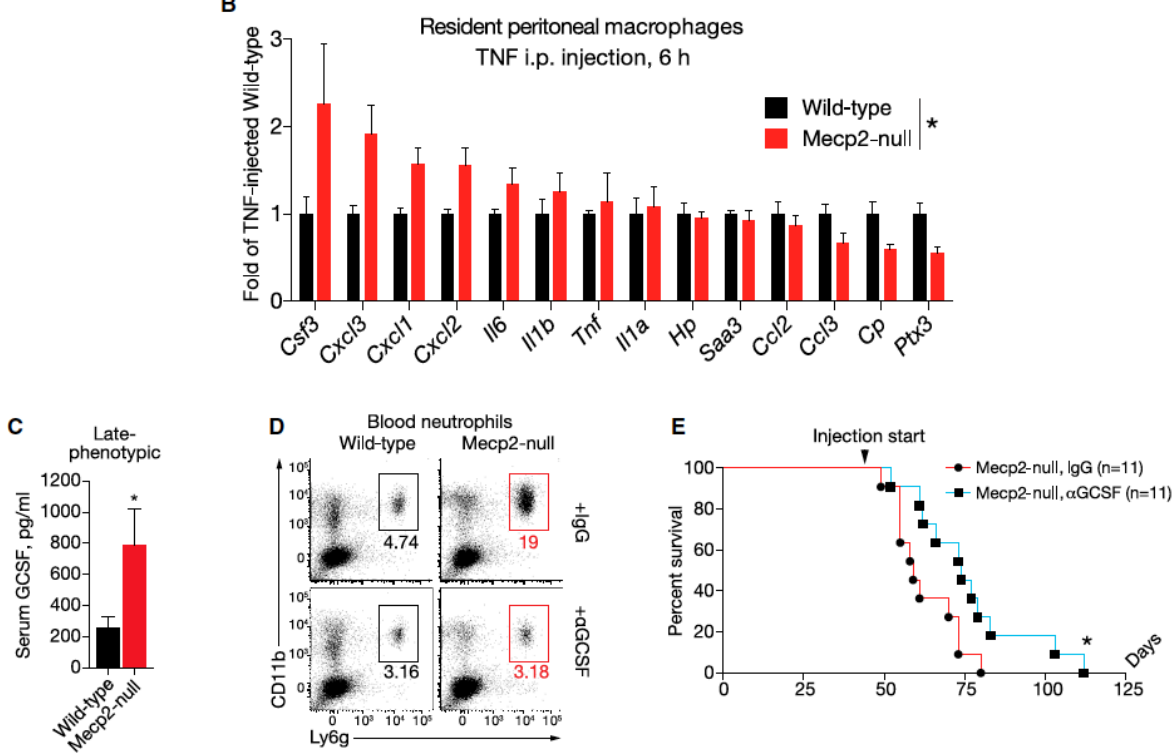

Figure 6. Mecp2 Restrains Inflammatory Responses in Macrophages

(A) Wild-type and Mecp2-null macrophages were treated for $6 \mathrm{hr}$ with TNF and subjected to quantitative real time PCR (qRT-PCR) for Tnf, II6, Cxc/2, $\mathrm{CxCl} / 3$, and Csf3. Data are representative of three experiments (average of three values $\pm \mathrm{SEM} ;{ }^{*+\cdots} \mathrm{p}<0.001$ and ${ }^{* *} \mathrm{p}<0.01$; Two-way ANOVA with Bonferroni post-test).

(B) Mecp2-null or age-matched wild-type mice were injected with intraperitoneal TNF and allowed to respond for $6 \mathrm{hr}$. Resident peritoneal macrophages were collected by lavage and subsequent AutoMACS sort for $\mathrm{F} / \mathrm{BO}^{+}$cells. RNA was collected and qRT-PCR performed for the indicated genes. One sample was removed due to significantly lower punty of macrophages ( $\sim 50 \%$ vs $70 \%-80 \%$ for all other samples; the removed sample was an outlier as measured by $>2$ SD below the mean for macrophage purity). ${ }^{*} \mathrm{p}<0.05$; two-way ANOVA with Bonferroni post-test. Interaction for Genotype and Gene; ${ }^{*} \mathrm{p}=0.01$. $\mathrm{N}=3$ wild-type and 4 Mecp2-null. Data are presented as mean \pm SEM.

(C) Granulocyte colony-stimulating factor (GCSF) EUSA of serum from late-phenotypic Mecp2-null mice and wild-type controls ( ${ }^{*} p<0.05$; Mann-Whitney twotailed; $n=9$ per group. Data are presented as mean $\pm S E M)$.

(D) Representative flow cytometry plots of Mecp2-null and wild-type mice treated with anti-GCSF neutralizing or isotype antibodies showing percentage of neutrophils out of all $\mathrm{CD}_{4} 5^{+}$circulating blood cells. Plots are representative of two independent experiments performed with $n=2$ mice for all groups, analyzed 1-2 weeks post start of injections.

(E) Survival of Mecp2-null mice treated with either anti-GCSF neutralizing or isotype antibodies ("p $<0.05$; log-rank Mantel-Cox test; $n=11$ per group).

688 Immunity 42, 679-691, April 21, 2015 @2015 Elsevier Inc. 
Appendix: Methyl-CpG binding protein 2 regulates microglia and macrophage gene expression in response to inflammatory stimuli

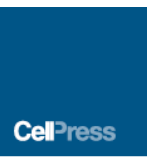

induced by maladaptive responses to stimuli by Mecp2-null macrophages. Such a positive feedback loop involving multiple cell types and tissues could help explain the progressive development of symptoms in Mecp2-null mice, which ultimately lead to death. These results might also explain why cell-specific deletion of Mecp2 in microglia cells did not result in significant symptoms of Rett syndrome (unpublished data); Mecp2-null microglia and macrophages represent amplifiers of pathology, driven primarily by neurons, and thus their replacement by wild-type cells abrogates the otherwise vicious cycle and slows down the disease progression; however, without the pathology driven by neurons (such as apneas, abnormal glucocorticoid response, etc.), Mecp2-null microglia cannot induce a Rett-like pathology.

It is well-recognized that girls suffering from Rett syndrome experience severe problems outside the CNS, such as gastrointestinal distention, defects of nutrient absorption, scoliosis, osteopenia, and general somatic growth deficits (Chahrour and Zoghbi, 2007; Dunn and MacLeod, 2001). Intestines contain a large number of immune cells, including a significant fraction of macrophages (Zigmond and Jung, 2013). Osteoclasts are key players in bone tumover and homeostasis (Davies et al., 2013). Thus, it is conceivable that pathology in both the gastrointestinal tract and the bones could be exacerbated by defects of tissueresident macrophage function and/or loss of tissue-resident macrophages in these tissues. It has been suggested that macrophages might be one of the two largest sources of insulin-like growth factor 1 (IGF-1), which is a critical growth factor involved in growth and health of a multitude of tissues (Gow et al., 2010). In addition, IGF-1 treatment has been shown to increase the lifespan of Mecp2-null mice (Tropea et al., 2009) and recently initiated clinical trials are promising (Khwaja et al., 2014). It is therefore possible that functional defects and loss of tissue-resident macrophages in Mecp2-deficient mice, and possibly Rett syndrome, might contribute to overall pathology via deficiency of macrophage-derived IGF-1. Moreover, our RNA-seq data shows that Mecp2-null microglia express increased amounts of Igfbp3, a binding protein for IGF-1, which binds soluble IGF-1 and neutralizes its function.

In recent groundbreaking work examining deletion of $M e c p 2$ in adult mice, phenotypic progression is similar in time-scale to that seen in mice null from birth (McGraw et al., 2011). This might support the hypothesis that accumulation of cellular stress in the absence of Mecp2 is progressively detrimental to cells necessary for tissue homeostasis, such as tissue-resident macrophages.

Beyond Rett syndrome, this work suggests that Mecp2 is an epigenetic regulator of macrophages. Given the diversity and plasticity of macrophages across organ systems, understanding the role of Mecp2 in each specific macrophage population might prove a difficult task. However, understanding the role of Mecp2 in macrophages, and the immune system as a whole, could lead to greater understanding of several diseases in addition to Rett syndrome, including MeCP2 duplication/triplication syndrome (Chahrour and Zoghbi, 2007), and systemic lupus erythematosus (Koelsch et al., 2013), which has been linked to abnormalities of MeCP2 in humans.

We found genetic dysregulation in response to multiple stimulations in Mecp2-null macrophages, which in vivo might act alone or in concert depending upon the exact conditions presented to the cell. For instance, many of the hypoxia-induced transcripts measured to be abnormal in Mecp2-null macrophages, were also found in the disrupted glucocorticoid pathways identified by RNA-Seq. Therefore, such transcripts might represent independent dysfunction in Mecp2-null macrophages in the context of hypoxia alone, or an additional complication of glucocorticoid-induced transcription depending on context. Further, the combination of glucocorticoid stimulation and hypoxia might result in complex transcriptional dysregulation.

Our validation studies of inflammatory-, hypoxia-, and glucocorticoid- signaling confim a macrophage-intrinsic role for Mecp2 in proper execution of these transcriptional responses. We validated in vitro that stimulation of Mecp2-null macrophages demonstrates excessive and dysregulated inflammatory response and excessive response to low-dose glucocorticoids, which have been previously shown to contribute to pathology in mouse models of Mecp2-deficiency (Braun et al., 2012). Of particular note, we found that Mecp2-null mice develop an inflammatory activation of microglia with disease progression, and that resident peritoneal macrophages stimulated with TNF in vivo, or BMDM stimulated with TNF in vitro, have an abnormal transcriptional response which implicates Mecp2 as a key regulator of inflammatory gene programs in macrophages. Of note, TNF is a widely used cytokine with important functions in both the CNS and PNS in the context of normal, non-inflammatory function and development (Stellwagen and Malenka, 2006; Wheeler et al., 2014). Based on what we have demonstrated in macrophages, it is possible that TNF signaling in neurons is also impaired in the absence of Mecp2, potentially contributing to abnomal neuronal function and/or development in Rett syndrome. This possible cascade will be evaluated in future studies.

These results provide evidence that Mecp2 plays an important role in the epigenetic regulation of macrophage responses and implicate macrophages as therapeutic targets in Rett syndrome. Importantly, we found that three stimuli (glucocorticoids, hypoxia, and inflammation) were transcriptionally dysfunctional in the absence of Mecp2, associating Mecp2 as a widely used epigenetic regulator in macrophages. Given that Mecp2 regulates multiple response pathways in macrophages, it is important to note that the exact effects of Mecp2-deficiency in macrophages in the context of a severe whole-body disease such as Rett syndrome becomes extremely difficult to predict, especially when one considers the variation in location, context, and functions of macrophages throughout the body. Thus, future studies should consider Mecp2 as an important player in the transcriptional regulation of macrophage responses and endeavor to understand the role of Mecp2 in the many unique macrophage populations.

EXPERIMENTAL PROCEDURES

Additional experimental procedures can be found in the Supplemental Information.

Animals

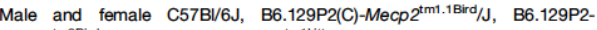

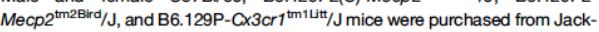
son Laboratories and/or bred in house using stock obtained from Jackson Laboratories. $\mathrm{C} \times 3 \mathrm{Cr}^{\mathrm{CreER}}$ mice were kindly provided by S. Jung (Weizmann

Immunity 42, 679-691, April 21, 2015 (2015 Elsevier Inc. 689 
Appendix: Methyl-CpG binding protein 2 regulates microglia and macrophage gene expression in response to inflammatory stimuli

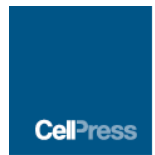

Institute of Science) and were maintained in our laboratory on C57BI/6J background. All animals were housed in temperature and humidity controlled rooms, and maintained on a $12 \mathrm{hr} / 12 \mathrm{hr}$ light/dark cycle (lights on at 7:00). All strains were kept in identical housing conditions. Mice were scored for $p$ a thology based on a 3-point scale across four categories: hind-limb-clasping, and 2 were considered early/pre-phenotypic. Approximate ages for prephenotypic mice were 4-5 weeks, and for late-phenotypic 8-12 weeks; however, all experiments were performed and labeled based on actual phenotype of the mice. All procedures complied with regulations of the Institutional Animal Care and Use Committee at The University of Virginia

Tamoxifen Treatment

Tamoxifen (Sigma T5648) was solubilized in corn oil (Sigma) at $10 \mathrm{mg} / \mathrm{ml}$. Mice were injected three times, subcutaneously, between the shoulder blades, at $48 \mathrm{hr}$ intervals at a dose of $100 \mathrm{mg} / \mathrm{kg}$. For tamoxifen feeding, mice were placed on tamoxifen diet TD.130856 (Harlan Laboratories) for up to 3 months placed on tamoxifen diet TD.130856 (Harlan Laboratories) for up to 3 months before analysis,
Laboratories).

RNA-Seq Analysis

Total RNA was extracted using the RNeasy mini kit (QIAGEN). RNA-seq was performed by Hudson Alpha Genomic Services Laboratory. Statistical analysis and data post-processing were performed with in-house developed functions in Matlab (Litvak et al., 2009; Litvak et al., 2012). For transcriptome analysis of wild-type and Mecp2-null microglia and peritoneal macrophages, genes were selected for inclusion on the basis of filtering for minimum log2 expression intensity $(>4)$.

Gene Set Enrichment Analysis

Gene set enrichment analysis (GSEA) is an analytical tool for relating differentially regulated genes to transcriptional signatures and molecular pathways associated with known biological functions (Subramanian et al., 2005). The statistical significance of the enrichment of known transcriptional signatures in a ranked list of genes was determined as described (Subramanian et al., 2005). To assess the phenotypic association with Mecp2 deficiency, we used the list of genes that was ranked according to differential gene expression in Mecp2-null and wild-type microglia. We used 4,722 gene sets from the Molecular Signature Database C2 version 4.0 and 32 custom gene sets including hypoxia and glucocorticoid-stimulated gene sets (Table S1).

Quantitative Chromatin Immunoprecipitation Analysis ForChIP analysis, formal in-fixed cells were sonicated and processed for immu noprecipitation essentially as described (Ning et al., 2011). In brief, $1.5 \times 10^{7}$ BMMs were cross-linked for $10 \mathrm{~min}$ in $1 \%$ paraformaldehyde, washed, and lysed. Chromatin was sheared by sonication $(5$ cycles $\times 60$ sec each cycle at $30 \%$ maximum potency) to fragments of approximately $150 \mathrm{bp}$. For Mecp2 ChIP, the sheared chromatin was incubated with anti-Mecp2 antibodies (ABE171) (Millipore), and then immunoprecipitated using anti-rabbit immuno(ABE171) (Millipore), and then immunoprecipitated using anti-rabbit immuno-
globulin G (IgG) Dynabeads (Invitrogen) pre-conjugated with anti-Chicken IgY globulin G (IgG) Dynabeads (Invitrogen) pre-conjugated with anti-Chicken IgY
antibodies (ab6877) (Abcam), washed, and eluted. For acH4 ChIP, the sheared antibodies (ab6877) (Abcam), washed, and eluted. For acH4 ChIP, the sheared chromatin was incubated with anti-rabbit lgG Dynabeads (Invitrogen) pre-con-
jugated with antibodies acH4 (06-598) (Upstate) or Isotype control IgG1 (BD jugated with antibodies acH4 (06-598) (Upstate) or Isotype control IgG1 (BD Phamigen), washed, and eluted. Eluted chromatin was reverse-cros

For quantitative ChIP, immunoprecipitated DNA samples were amplified with Fkbp5-promoter-specific primers (Forward: TGCACTGCCTATGCAAA TGA and Peverse: AGCTTCCTCCATCCCTCTI) using TaqMan quantitative PCR analysis. PCR samples from IgY-ChIPs served as a negative control.

ACCESSION NUMBERS

The Gene Expression Omnibus (GEO) accession number for the RNA-seq data reported in this paper is GSE66211, and the Gene Expression Omnibus (GEO) accession number for the ChIP-seq data reported in this paper is GSE66501.

690 Immunity 42, 679-691, April 21, 2015 @2015 Elsevier Inc.
SUPPLEMENTAL INFORMATION

Supplemental Information includes five figures, Supplemental Experimental Procedures, three tables, and two movies and can be found with this article online at http://dx.doi.org/10.1016/.j.immuni.2015.03.013.

AUTHOR CONTRIBUTIONS

J.C.C. and N.C.D. designed the experiments, performed the experiments, analyzed the data, and wrote the manuscript. E.J. assisted with flow cytometry and immunohistochemistry experiments. Y.X. analyzed RNA-seq data and performed in vitro experiments. A.L. performed in vitro experiments. I.S. participated in all experiments with experimental animals. W.B. assisted with maintenance and genotyping of experimental animals. G.T.N. performed the microglia Sholl analysis. IM. assisted with intestinal preparations. N.C. assisted with in vitro experiments. A. provided ChIP-seg dato Y.W. and S. enerated the transgenic ex or. ments, analyzed data, and wrote the manuscript. J.K. conceived and led the project, designed the experiments, and wrote the manuscript.

\section{ACKNOWLEDGMENTS}

We thank Shirley Smith for editorial assistance and Dr. Alex Koeppel (Bioinformatics Core, School of Medicine, University of Virginia) for his initial analysis of BNA-seq data. We thank Dr. Arthur Mercurio and Bryan Pursell (Department of Molecular, Cell and Cancer Biology. UMass Medical School) for their help with hypoxio ypoxia val their valuable comments during multiple discussions of this work. N.C.D. was supported by Hartwell Foundation post-doctoral fellowship. J.C.C. was supported by an award from the National Institute of Allergy and Infectious Diseases IF30Al109984. This work was primarily supported by a grant from the National Institutes of Neurological Disorders and Stroke NS081026 (J. K.), the Simons Foundation Autism Research Initiative (J. K.), and from the Rett Syndrome Research Trust (J. K.).

Received: April 28, 2014 Revised: November 25, 2014
Accepted: February 15, 2015 Published: April 21, 2015

REFERENCES

Amir, R.E., Van den Veyver, I.B., Wan, M., Tran, C.Q., Francke, U., and Zoghbi, H.Y. (1999). Rett syndrome is caused by mutations in X-linked MECP2, encoding methyl-CpG-binding protein 2. Nat. Genet. 23, 185-188.

Bain, C.C., Scott, C.L., Uronen-Hansson, H., Gudjonsson, S., Jansson, O. Grip, O., Guilliams, M., Malissen, B., Agace, W.W., and Mowat, A.M. (2013). Resident and pro-inflammatory macrophages in the colon represent altemative context-dependent fates of the same Ly6Chi monocyte precursors. Mucosal Immunol. 6, 498-510.

Ballas, N., Lioy, D.T., Grunseich, C., and Mandel, G. (2009). Non-cell autonomous influence of MeCP2-deficient glia on neuronal dendritic morphology. Nat. Neurosci. 12, 311-317.

Bendall, LJ., and Bradstock, K.F. (2014). G-CSF: From granulopoietic stimulant to bone marrow stem cell mobilizing agent. Cytokine Growth Factor Rev. 25, 355-367.

Braun, S., Kottwitz, D., and Nuber, U.A. (2012). Pharmacological interference with the glucocorticoid system influences symptoms and lifespan in a mouse model of Rett syndrome. Hum. Mol. Genet. 21, 1673-1680.

Butovsky, O., Jedrychowski, M.P., Moore, C.S., Cialic, R., Lanser, A.J., Gabriely, G., Koeglsperger, T., Dake, B., Wu, P.M., Doykan, C.E., et al. (2014). Identification of a unique TGF- $\beta$-dependent molecular and functional signature in microglia. Nat. Neurosci. 17, 131-143.

Chahrour, M., and Zoghbi, H.Y. (2007). The story of Rett syndrome: from clinic to neurobiology. Neuron $56,422-437$. 
Appendix: Methyl-CpG binding protein 2 regulates microglia and macrophage gene expression in response to inflammatory stimuli

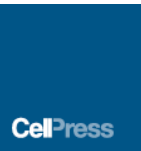

Dai, X.M., Ryan, G.R., Hapel, A.J., Dominguez, M.G., Russell, R.G., Kapp, S., Sylvestre, V., and Stanley, E.R. (2002). Targeted disnuption of the mouse colony-stimulating factor 1 receptor gene results in osteopetrosis, mononuclear phagocyte deficiency, increased primitive progenitor cell frequencies, and reproductive defects. Blood $99,111-120$

Davies, L.C., Jenkins, S.J., Allen, J.E., and Taylor, P.R. (2013). Tissue-resident macrophages. Nat. Immunol. 14, 986-995.

Derecki, N.C., Cronk, J.C., Lu, Z., Xu, E., Abbott, S.B., Guyenet, P.G., and Kipnis, J. (2012). Wild-type microglia arrest pathology in a mouse model of Rett syndrome. Nature 484, 105-109.

Dunn, H.G., and MacLeod, P.M. (2001). Rett syndrome: review of biological abnomalities. Can. J. Neurol. Sci. 28, 16-29.

Ebert, D.H., Gabel, H.W., Robinson, N.D., Kastan, N.R., Hu, LS., Cohen, S., Navarro, A.J., Lyst, M.J., Ekiert, R., Bird, A.P., and Greenberg, M.E. (2013). Activity-dependent phosphorylation of MeCP2 threonine 308 regulates interaction with NCoR. Nature 499, 341-345.

Ginhoux, F., Greter, M., Leboeuf, M., Nandi, S., See, P., Gokhan, S., Mehler, M.F., Conway, S.J., Ng, LG., Stanley, E.R., et al. (2010). Fate mapping analysis reveals that adult microglia derive from primitive macrophages. Science 330 , 841-845.

Goldmann, T., Wieghofer, P., Müller, P.F., Wolf, Y., Varol, D., Yona, S., Brendecke, S.M., Kierdorf, K., Staszewski, O., Datta, M., et al. (2013). A new type of microglia gene targeting shows TAK1 to be pivotal in CNS autoimmune inflammation. Nat. Neurosci. 16, 1618-1626.

Gow, D.J., Sester, D.P., and Hume, D.A. (2010). CSF-1, IGF-1, and the control of postnatal growth and development. J. Leukoc. Biol. 88, 475-481.

Guy, J., Cheval, H., Selfridge, J., and Bird, A. (2011). The role of MeCP2 in the brain. Annu. Rev. Cell Dev. Biol. 27, 631-652.

Hashimoto, D., Chow, A., Noizat, C., Teo, P., Beasley, M.B., Lebouf, M., Becker, C.D., See, P., Price, J., Lucas, D., et al. (2013). Tissue-resident macrophages self-maintain locally throughout adult life with minimal contribution from circulating monocytes. Immunity 38, 792-804.

Jung, S., Aliberti, J., Graemmel, P., Sunshine, M.J., Kreutzberg, G.W., Sher, A., and Littman, D.R. (2000). Analysis of fractalkine receptor CX(3)CR1 function by targeted deletion and green fluorescent protein reporter gene insertion. Mol. Cell. Biol. 20, 4106-4114.

Khwaja, O.S., Ho, E., Bames, K.V., O'Leary, H.M., Pereira, L.M., Finkelstein, Y., Nelson, C.A., 3rd, Vogel-Farley, V., DeGregorio, G., Holm, I.A., et al. (2014). Safety, pharmacokinetics, and preliminary assessment of efficacy of mecasermin (recombinant human IGF-1) for the treatment of Rett syndrome. Proc. Natl. Acad. Sci. USA 111, 4596-4601.

Kierdorf, K., Emy, D., Goldmann, T., Sander, V., Schulz, C., Perdiguero, E.G., Wieghofer, P., Heinnich, A., Riemke, P., Hölscher, C., et al. (2013). Microglia emerge from erythromyeloid precursors via Pu.1- and Irf-dependent pathways. Nat. Neurosci. 16, 273-280.

Koelsch, K.A., Webb, R., Jeffries, M., Dozmorov, M.G., Frank, M.B., Guthridge, J.M., James, J A., Wren, J.D., and Sawalha, A.H. (2013). Functional characterization of the MECP2/IRAK1 lupus risk haplotype in human T cells and a human MECP2 transgenic mouse. J. Autoimmun. 41, 168-174.

Kozlowski, C., and Weimer, R.M. (2012). An automated method to quantify microglia morphology and application to monitor activation state longitudinally in vivo. PloS one 7, e31814.

Lioy, D.T., Garg, S.K., Monaghan, C.E., Raber, J., Foust, K.D., Kaspar, B.K., Hirrlinger, P.G., Kirchhoff, F., Bissonnette, J.M., Ballas, N., and Mandel, G. (2011). A role for glia in the progression of Rett's syndrome. Nature 475, 497-500.

Litvak, V., Ramsey, S.A., Rust, A.G., Zak, D.E., Kennedy, K.A., Lampano, A.E., Nykter, M., Shmulevich, l., and Aderem, A. (2009). Function of C/EBPdelta in a regulatory circuit that discriminates between transient and persistent TLR4induced signals. Nat. Immunol. 10, 437-443.

Litvak, V., Ratushny, A.V., Lampano, A.E., Schmitz, F., Huang, A.C., Raman, A., Rust, A.G., Bergthaler, A., Aitchison, J.D., and Aderem, A. (2012). A FOXO3-IRF7 gene regulatory circuit limits inflammatory sequelae of antiviral responses. Nature $490,421-425$.
Lyst, M.J., Ekiert, R., Ebert, D.H., Merusi, C., Nowak, J., Selfnidge, J., Guy, J., Kastan, N.R., Robinson, N.D., de Lima Alves, F., et al. (2013). Rett syndrome mutations abolish the interaction of MeCP2 with the NCoR/SMRT corepressor. Nat. Neurosci. 16, 898-902.

Maezawa, I., and Jin, L.W. (2010). Rett syndrome microglia damage dendrites and synapses by the elevated release of glutamate. J. Neurosci. 30, 53465356.

McGraw, C.M., Samaco, R.C., and Zoghbi, H.Y. (2011). Adult neural function requires MeCP2. Science 333, 186.

Nguyen, M.V., Felice, C.A., Du, F., Covey, M.V., Robinson, J.K., Mandel, G., and Ballas, N. (2013). Oligodendrocyte lineage cells contribute unique features to Rett syndrome neuropathology. The Journal of neuroscience: the official joumal of the Society for Neuroscience 33, 18764-18774.

Ning, S., Pagano, J.S., and Barber, G.N. (2011). IRF7: activation, regulation, modification and function. Genes Immun. 12, 399-414.

Nuber, U.A., Kriaucionis, S., Roloff, T.C., Guy, J., Selfridge, J., Steinhoff, C., Schulz, R., Lipkowitz, B., Ropers, H.H., Holmes, M.C., and Bird, A. (2005). Up-regulation of glucocorticoid-regulated genes in a mouse model of Rett syndrome. Hum. Mol. Genet. 14, 2247-2256.

Schulz, C., Gomez Perdiguero, E., Chorro, L, Szabo-Rogers, H., Cagnard, N. Kierdorf, K., Prinz, M., Wu, B., Jacobsen, S.E., Pollard, J.W., et al. (2012). A lineage of myeloid cells independent of Myb and hematopoietic stem cells. Science 336, 86-90.

Shahbazian, M.D., Antalffy, B., Armstrong, D.L., and Zoghbi, H.Y. (2002). Insight into Rett syndrome: MeCP2 levels display tissue- and cell-specific differences and correlate with neuronal maturation. Hum. Mol. Genet. 11, 115-124.

Sica, A., and Mantovani, A. (2012). Macrophage plasticity and polarization: in vivo veritas. J. Clin. Invest. 122, 787-795.

Stellwagen, D., and Malenka, R.C. (2006). Synaptic scaling mediated by glial TNF-alpha. Nature 440, 1054-1059.

Subramanian, A., Tamayo, P., Mootha, V.K., Mukheriee, S., Ebert, B.L. Gillette, M.A., Paulovich, A., Pomeroy, S.L, Golub, T.R., Lander, E.S., and Mesirov, J.P. (2005). Gene set enrichment analysis: a knowledge-based approach for interpreting genome-wide expression profiles. Proc. Natl. Acad. Sci. USA 102, 15545-15550.

Sunderkötter, C., Nikolic, T., Dillon, M.J., Van Rooijen, N., Stehling, M., Drevets, D.A., and Leenen, P.J. (2004). Subpopulations of mouse blood monocytes differ in maturation stage and inflammatory response. J. Immunol. 172, 4410-4417.

Tropea, D., Giacometti, E., Wilson, N.R., Beard, C., McCurry, C., Fu, D.D., Flannery, R., Jaenisch, R., and Sur, M. (2009). Partial reversal of Rett Syndrome-like symptoms in MeCP2 mutant mice. Proc. Natl. Acad. Sci. USA 106, 2029-2034.

Urdinguio, R.G., Lopez-Serra, L., Lopez-Nieva, P., Alaminos, M., Diaz-Uriarte, R., Femandez, A.F., and Esteller, M. (2008). Mecp2-null mice provide new neuronal targets for Rett syndrome. PLOS ONE 3, e3669.

Varol, C., Vallon-Eberhard, A., Elinav, E., Aychek, T., Shapira, Y., Luche, H., Fehling, H.J., Hardt, W.D., Shakhar, G., and Jung, S. (2009). Intestinal lamina propria dendritic cell subsets have different origin and functions. Immunity 31 , 502-512.

Wheeler, M.A., Heffner, D.L., Kim, S., Espy, S.M., Spano, A.J., Cleland, C.L., and Deppmann, C.D. (2014). TNF- $\alpha$ /TNFR1 signaling is required for the development and function of primary nociceptors. Neuron $82,587-602$.

Yasui, D.H., Xu, H., Dunaway, K.W., Lasalle, J.M., Jin, LW., and Maezawa, I. (2013). MeCP2 modulates gene expression pathways in astrocytes. Mol Autism 4, 3

Yona, S., Kim, K.W., Wolf, Y., Mildner, A., Varol, D., Breker, M., Strauss-Ayali, D., Viukov, S., Guilliams, M., Misharin, A., et al. (2013). Fate mapping reveals origins and dynamics of monocytes and tissue macrophages under homeostasis. Immunity $38,79-91$.

Zigmond, E., and Jung, S. (2013). Intestinal macrophages: well educated exceptions from the rule. Trends Immunol. 34, 162-168. 
Appendix: Methyl-CpG binding protein 2 regulates microglia and macrophage gene expression in response to inflammatory stimuli

Immunity

Supplemental Information

\section{Methyl-CpG Binding Protein 2 Regulates}

\section{Microglia and Macrophage Gene Expression}

in Response to Inflammatory Stimuli

James C. Cronk, Noël C. Derecki, Emily Ji, Yang Xu, Aaron Lampano, Igor Smirnov, Wendy Baker, Geoffrey T. Norris, loana Marin, Nathan Coddington, Yochai Wolf,

Stephen D. Turner, Alan Aderem, Alexander L. Klibanov, Tajie H. Harris, Steffen Jung, Vladimir Litvak, and Jonathan Kipnis 
Appendix: Methyl-CpG binding protein 2 regulates microglia and macrophage gene expression in response to inflammatory stimuli

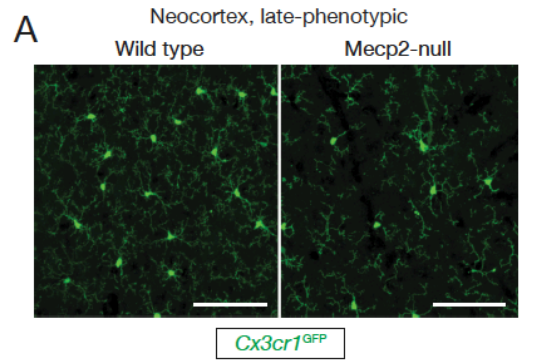

Figure S1, related to Figure 1.

Representative images of wild type and late-phenotypic Mecp2-null microglia.

(A, B) Representative images from Cx3 $\mathrm{crl}^{\mathrm{GFP} /+}$ or non-GFP (Ibal staining) wild type and late-phenotypic Mecp2-null

B Cerebellum, late-phenotypic brains (scale bars: (A), $75 \mu \mathrm{m}$; (B), 120

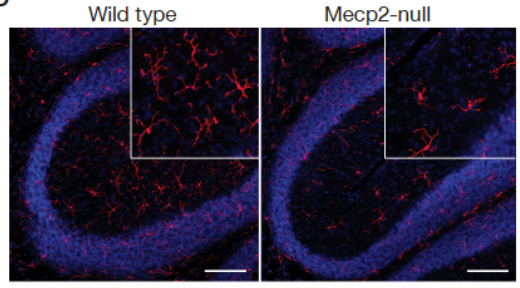

\begin{tabular}{|l|l|l|}
\hline IBA-1 & DAPI \\
\hline
\end{tabular}

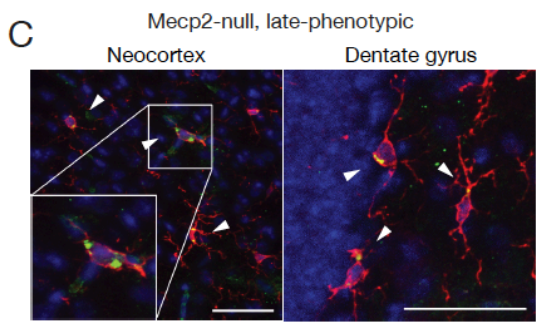
images to allow for better visualization of microglial morphology.

(C) Iba1 and cleaved caspase $3(\mathrm{CC} 3)$ staining in late-phenotypic Mecp2-null brains demonstrating $\mathrm{CC}^{+}$microglia (scale bar, $40 \mu \mathrm{m}$ ). Images were cropped from larger images to allow for better visualization of $\mathrm{CC} 3$ localization within microglia. 
Appendix: Methyl-CpG binding protein 2 regulates microglia and macrophage

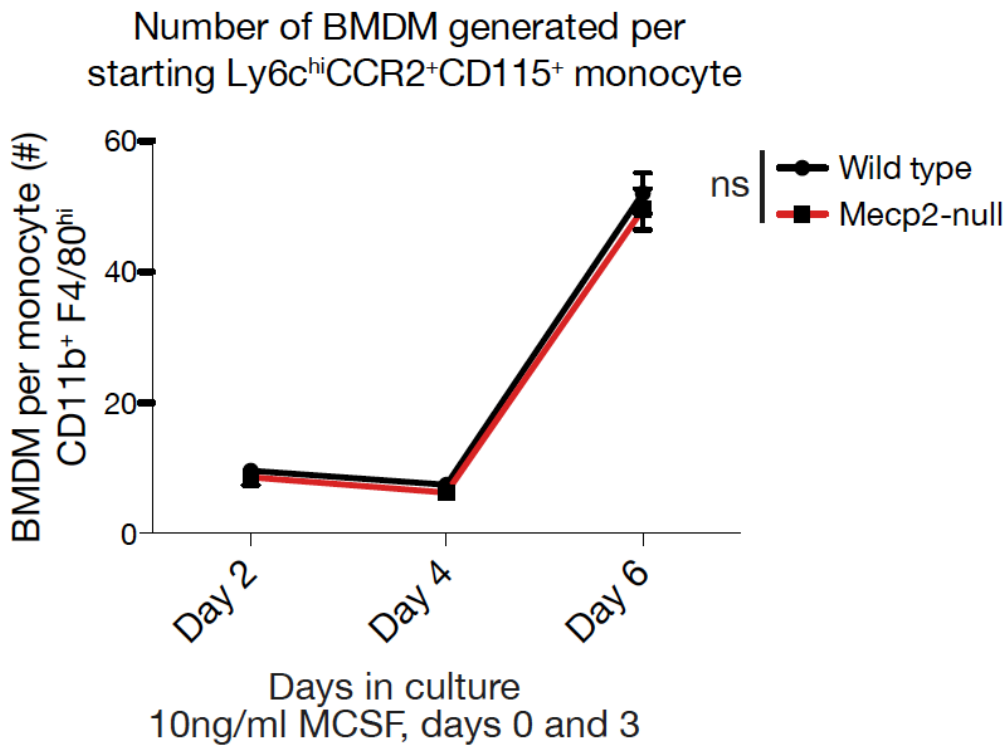

Figure S2, related to Figure 3. BMDM generated per monocyte in wild type and Mecp2-

null. Wild type and Mecp2-null BMDM were generated as described. Briefly, equal numbers of live bone marrow cells were plated in $10 \mathrm{ng} / \mathrm{ml} \mathrm{MCSF}$ for 3 days, then given $50 \%$ additional volume of media containing $20 \mathrm{ng} / \mathrm{ml}$ MCSF for an additional 3 days. Cell counts were obtained from cells generated from $\mathrm{n}=3$ wild type and $\mathrm{n}=3$ Mecp2-null mice on days 2, 4, and 6 during culture. Flow cytometric analysis of bone marrow prior to culture was performed to obtain the percentage of cells plated that were $\mathrm{Ly} 6 \mathrm{C}^{\mathrm{hi}} \mathrm{CD} 115^{+} \mathrm{CD} 11 \mathrm{~b}^{+}$monocyte progenitors. This percentage was multiplied by the total number of cells plated to obtain the actual number of starting monocyte progenitors in each culture dish. The number of BMDM $\left(\mathrm{CD} 11 \mathrm{~b}^{+} \mathrm{F} 4 / 80^{\mathrm{hi}}\right)$ counted on each day of development was divided by the number of starting monocytes to calculate the number of macrophages derived per monocyte. Two-way ANOVA with Bonferroni post-test, not significant. Data represent mean \pm SEM. 
Appendix: Methyl-CpG binding protein 2 regulates microglia and macrophage gene expression in response to inflammatory stimuli
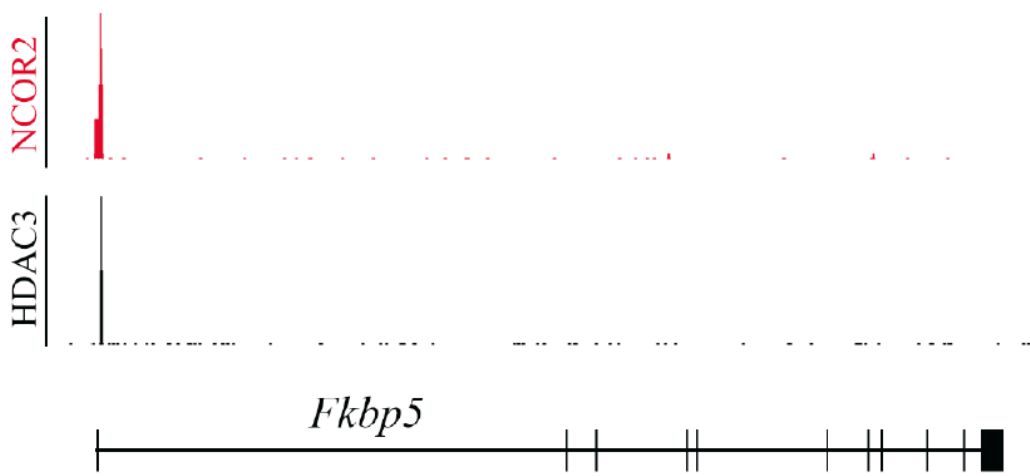

Chr. $17 \quad 28,620,000 \quad 28,610,000 \quad 28,600,000 \quad 28,590,000 \quad 28,580,000 \quad 28,570,000 \quad 28,560,000 \quad 28,550,000 \quad 28,540,000$

Figure S3, related to Figure 5. NCOR2 and HDAC3 occupancy profiles at the Fkbp5 gene promoter. ChIP-Seq analysis demonstrates NCOR2 and HDAC3 binding profiles at Fkbp 5 gene promoter in wild type bone marrow derived macrophages. The genomic region surrounding the Fkbp5 gene locus is shown, for which NCOR2 and HDAC3 occupancies are shown in red and black, respectively. Normalized counts, tag coverage per base per $10^{6}$ sequenced reads, are indicated on the Y-axis. Data are representative of two experiments. 
Appendix: Methyl-CpG binding protein 2 regulates microglia and macrophage gene expression in response to inflammatory stimuli

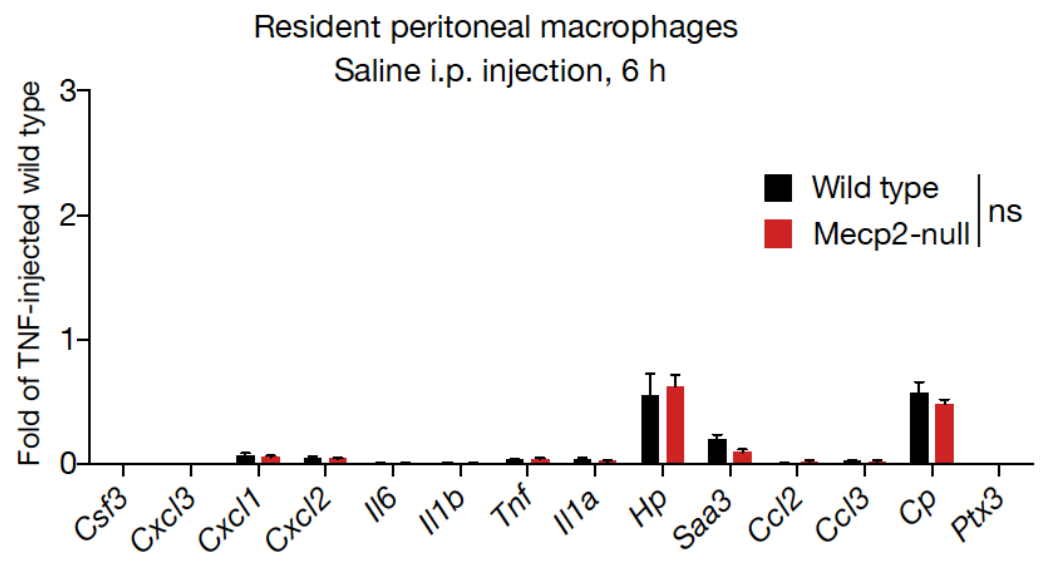

Figure S4, related to Figure 6. Intraperitoneal saline injection into Mecp2-null mice does

not effect gene expression of TNF-induced transcripts. Mice were injected with $10 \mu 1$ saline per gram of mouse weight (equivalent volume to that injected in Figure 6B), allowed to respond for $6 \mathrm{~h}$, and then cells were collected by peritoneal lavage and positively selected for F4/80 via AutoMACS. RNA was collected and qRT-PCR performed. Gene expression was normalized to wild type expression after TNF treatment (see Figure 6B). No significant difference was detected by two-way ANOVA with Bonferroni post-test, $\mathrm{n}=3$ mice per group. Data represent mean \pm SEM. 
Appendix: Methyl-CpG binding protein 2 regulates microglia and macrophage gene expression in response to inflammatory stimuli

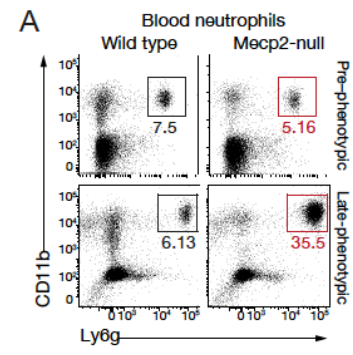

B
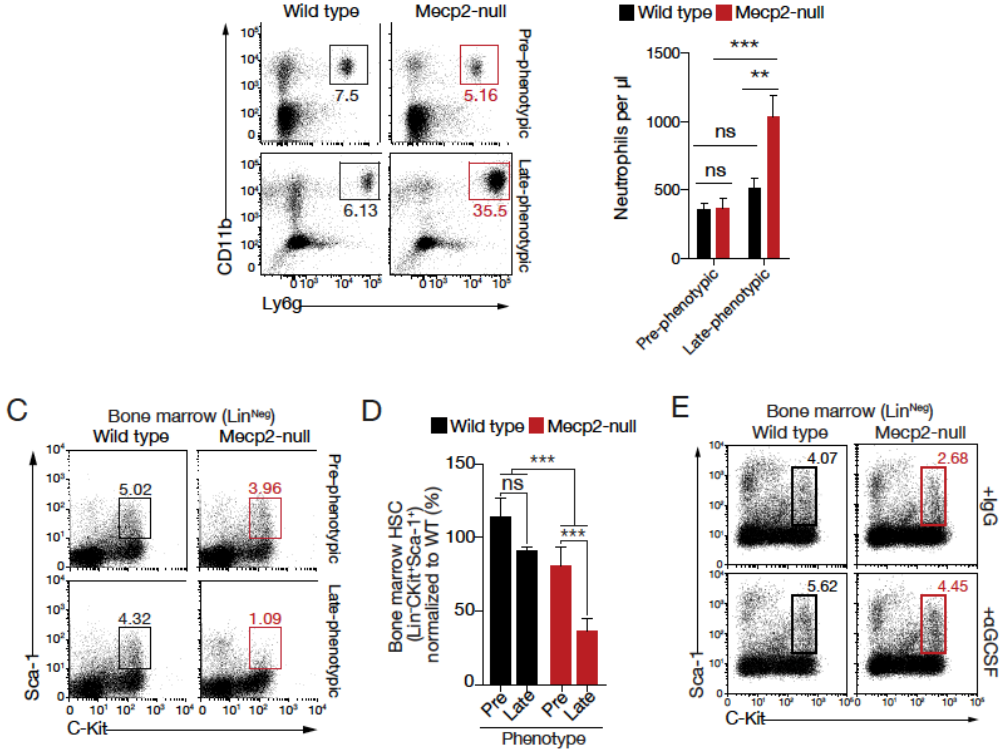

Figure S5, related to Figure 6. Mecp2-null mice have progressive neutrophilia and hematopoietic stem cell loss, which can be attenuated by anti-GCSF neutralizing antibodies.

(A, B) Neutrophil counts in Mecp2-null and age-matched wild types in pre- and late-phenotypic mice. Quantified by flow cytometry and total live cell counts from blood samples. (Two-way ANOVA with Bonferroni post-test; $\mathrm{n}=6-9$ mice per group; **, $\mathrm{p}<0.01 ; * * *, \mathrm{p}<0.001$. Data represent mean \pm SEM).

(C, D) Quantification of hematopoietic stem cells $\left(\mathrm{Lin}^{-} \mathrm{C}-\mathrm{Kit}^{+} \mathrm{Sca}-1^{+}\right)$in Mecp2-null and agematched wild type mice in BM isolates as measured by flow cytometry in pre- and latephenotypic mice $\left(\mathrm{Lin}^{\text {neg }} \mathrm{CKit}^{+} \mathrm{Sca}-1^{+}\right)(* * *, \mathrm{p}<0.001$; Two-way ANOVA with Bonferroni posttest. Data represent mean $\pm \mathrm{SEM}$ )

(E) Flow cyotmetric plots of hematopoietic stem cells $\left(\mathrm{Lin}^{-} \mathrm{C}-\mathrm{Kit}^{+} \mathrm{Sca}-1^{+}\right)$in Mecp2-null and wild type mice treated with anti-GCSF neutralizing antibodies or isotype control. 
Appendix: Methyl-CpG binding protein 2 regulates microglia and macrophage gene expression in response to inflammatory stimuli

\section{Supplemental Movies}

Movies were captured over 20 minutes at $1024 \times 1024$ and 22um depth, ( $\mathrm{Z}$ depth $22 \mathrm{um}$, step of lum) and cropped in Imaris to a width of $800 \times 800$ pixels, subjected to the following two filters:

Background subtraction, filter width of $22 \mathrm{um}$

Gaussian Filter, filter width of $0.01 \mathrm{um}$

Processed files were saved as Quicktime movies.

$\mathrm{Cx} 3 \mathrm{Cr}^{\mathrm{GFP} /+}$ (microglia; green), intravascular tracer (Qdot655; red)

Movie S1, related to Figure 1. Representative wild type microglia.

Movie S2, related to Figure 1. Representative Mecp2-null microglia from phenotypic mice. 
Appendix: Methyl-CpG binding protein 2 regulates microglia and macrophage

\section{Supplemental Tables}

Table S1, related to Figure 5. Gene set Signatures

Shown is the list of custom gene sets of hypoxia and glucocorticoid transcriptional signatures. These signatures were derived from the indicated public available databases.

Table S2, related to Figure 5. Transcriptional signatures enriched in Mecp2-null cells

Shown is the summary of gene set enrichment analysis (GSEA) that indicate the statistical scores of the enrichment of hypoxia, glucocorticoid and TNF-induced transcriptional signature genes in the transcriptome of Mecp2-null microglia cells.

Table S3, related to Figure 5. Glucocorticoid Signature Genes Up-regulated in Mecp2-null cells

Shown is the list of the Glucocorticoid Signature Genes that are up-regulated in microglia and peritoneal macrophages isolated from Mecp2-null mice. 
Appendix: Methyl-CpG binding protein 2 regulates microglia and macrophage

gene expression in response to inflammatory stimuli

\section{Supplemental Experimental Procedures}

\section{Flow cytometry}

Single cell suspensions were prepared by passing cells through a $70 \mu \mathrm{m}$ filter prior to staining. Cells were pelleted and resuspended in flow cytometry buffer ( $0.01 \mathrm{M}$ PBS, $1 \%$ BSA, $2 \mathrm{mM}$ EDTA, $0.1 \%$ sodium azide) containing $300 \mathrm{mg} / \mathrm{ml}$ mouse IgG as a blocking agent. Cells were then stained with the appropriate dilution of antibody or fluorescent label for $30 \mathrm{~m}$ at $4^{\circ} \mathrm{C}$ in the dark. In general, antibodies were obtained from eBioscience or BD. CCR2-FITC was obtained from R\&D Systems. Viability dye and anti-Ly6g antibodies were obtained from Biolegend. After staining, cells were washed in $3 \mathrm{ml}$ flow cytometry buffer, pelleted, and fixed in either $1 \%$ PFA or, for intranuclear staining, cells were prepared via manufacturer's instructions using the eBioscience Foxp3/Transcription Factor Staining Buffer Set. Cells were stained with either 1:1000 Chicken-anti-MeCP2 antibody (Millipore, ABE171) or rabbit-anti-Mecp2 (a kind gift from the laboratory of Gail Mandel) in permeabilization buffer (eBioscience) for $30 \mathrm{~m}$ at $4^{\circ} \mathrm{C}$ in the dark. Cells were washed in $3 \mathrm{ml}$ permeabilization buffer, pelleted, and then stained with the appropriate fluorescently-labeled secondary antibody (Invitrogen) at 1:1000 in permeabilization buffer for $30 \mathrm{~m}$ at $4^{\circ} \mathrm{C}$ in the dark. Cells were washed in $3 \mathrm{ml}$ permeabilization buffer, pelleted, and resuspended in 0.01 M PBS for analysis on a flow cytometer. 
Appendix: Methyl-CpG binding protein 2 regulates microglia and macrophage

\section{Preparation of brain samples}

Mice were perfused intracardially with $0.01 \mathrm{M}$ PBS containing $5 \mathrm{u} / \mathrm{ml}$ heparin until thoroughly exsanguinated to minimize peripheral immune cell contamination. The entire brain was then removed and meninges and choroid plexus carefully separated and discarded from the rest of the brain tissue. The brain was then minced gently using forceps and scalpel to begin dissociation of the tissue. Each brain was placed in $5 \mathrm{ml}$ HBSS (containing $\mathrm{Mg}$ and $\mathrm{Ca}$ ), $2 \mathrm{mg} / \mathrm{ml}$ papain, $50 \mathrm{u} / \mathrm{ml} \mathrm{DNASE-I} \mathrm{(Sigma)} \mathrm{and}$ Glutamax (Invitrogen) in a $15 \mathrm{ml}$ tube. Samples were then incubated at $37^{\circ} \mathrm{C}$ for $15 \mathrm{~m}$, followed by gentle trituration five times up and down with a $5 \mathrm{ml}$ plastic tissue culture pipette. Samples were again incubated at $37^{\circ} \mathrm{C}$ for $15 \mathrm{~m}$, followed by a second gentle trituration five times up and down with a $5 \mathrm{ml}$ plastic tissue culture pipette. Samples were incubated at $37^{\circ} \mathrm{C}$ for $15 \mathrm{~m}$ a third time, followed by gentle trituration ten times with a 9" heat-polished glass Pasteur pipette. At this point, cells were well dissociated. Each $15 \mathrm{ml}$ tube was filled to the top with DMEM/F12/10\% FBS and filtered gently through a $70 \mu \mathrm{m}$ cell strainer. Cells were then pelleted at $290 \mathrm{RCF}, 10 \mathrm{~m}$, with a slow brake. Supernatant was removed and cells were used for flow cytometric analysis or RNA collection.

\section{Preparation of brain suspension for RNA collection}

After initial processing to create single-cell brain suspensions, samples were resuspended in $2.1 \mathrm{ml}$ of $1.122 \mathrm{~g} / \mathrm{ml}$ isotonic Percoll (Sigma) + $7.9 \mathrm{ml} \mathrm{PBS}$. This mixture was underlayed with $2 \mathrm{ml} 1.088 \mathrm{~g} / \mathrm{ml}$ isotonic Percoll. The samples were then centrifuged for $30 \mathrm{~m}$ at $1159 \mathrm{RCF}$ at RT, slow start, and no brake. Myelin debris at the top of the gradient was then removed. The liquid (but not the pellet) was then transferred to a $50 \mathrm{ml}$ 
Appendix: Methyl-CpG binding protein 2 regulates microglia and macrophage

gene expression in response to inflammatory stimuli

conical tube. The liquid was washed with DMEM/F12/10\% FBS, pelleted, and then labeled with microglia $\mathrm{CD}_{1} 1 \mathrm{~b}^{+}$magnetic selection beads (Miltenyi). Cells were positively selected by AutoMACS. Flow cytometry of AutoMACS sorted cells isolated by this method and gated on singlet events, excluding debris, were $\sim 85 \%$ pure $\mathrm{CD} 45^{10} \mathrm{CD} 11 \mathrm{~b}^{+}$microglia. RNA was collected by RNeasy mini kit (Qiagen).

\section{Multiphoton microscopy}

Microglia were imaged through thinned skull. Briefly, mice were anesthetized with isoflurane and the skull thinned using a dental drill and microblade. Multiphoton imaging was performed using a Leica TCS SP8 multiphoton system equipped with a Coherent Chameleon Ti:Sapphire laser. GFP was excited with a wavelength of $880 \mathrm{~nm}$. Images were collected from a z-series with a depth of approximately 20 microns with a step size of 1 micron over 20 microns. Movies were generated and image analysis was performed using ImageJ.

\section{Immunohistochemistry}

Mice were euthanized and perfused with $5 \mathrm{u} / \mathrm{ml}$ heparinized PBS followed by $4 \%$ PFA in $0.01 \mathrm{M}$ PBS. Organs were removed and post-fixed in $4 \%$ PFA in $0.01 \mathrm{M}$ PBS at $4^{\circ} \mathrm{C}(2$ days for brain). Tissue was then cryoprotected by equilibrating in $30 \%$ sucrose, followed by slicing on a cryostat. Brain slices were labeled free-floating; spleen slices were mounted onto gelatin-coated charged slides. Meninges were fixed with 1:1 acetone/ethanol within the skull cap in situ at $-20 \mathrm{C}$ for $20 \mathrm{~min}$, washed with PBS, stained, carefully removed and whole mounted on slides for imaging. Tissue was blocked for $1 \mathrm{~h}$ 
Appendix: Methyl-CpG binding protein 2 regulates microglia and macrophage

gene expression in response to inflammatory stimuli

RT in 0.01 M PBS containing $0.1 \%$ Triton X-100 and $10 \%$ of the appropriate serum for secondary antibody staining. Slices were then incubated for $24 \mathrm{~h}$ (or $48 \mathrm{~h}$ for MeCP2 staining) in the dark at $4^{\circ} \mathrm{C}$ using the indicated antibodies in $0.01 \mathrm{M}$ PBS containing $0.01 \%$ sodium azide, $0.5 \%$ Triton X-100 and $2 \mathrm{mg} / \mathrm{ml}$ BSA. Antibodies used were 1:1000 Chicken-anti-MeCP2 (Millipore), 1:300 Goat-anti-Ibal (Abcam), 1:300 Rat-anti-CD169 (Biorad), 1:300 Rabbit-anti-CC3 (Cell Signaling), 1:100 Rabbit-anti-CD163 (Santa Cruz), 1:200 biotinylated-Rat-anti-F4/80 (AbD Serotec), and 1:100 a647-anti-CD31 (BD Biosciences). After staining, slices were washed with $0.01 \mathrm{M}$ PBS 3 times for $5 \mathrm{~m}$ on a shaker. Tissue was then incubated for $1 \mathrm{~h}$ at $\mathrm{RT}$ with the appropriate fluorescent secondary antibodies (Invitrogen), or streptavidin fluorescent conjugate (BD Biosciences) at 1:1000 in the same buffer used for primary antibody staining. Brain slices or meninges were then stained for $1 \mathrm{~m}$ in 1:20,000 DAPI diluted in PBS, washed with $0.01 \mathrm{M}$ PBS 3 times for $5 \mathrm{~m}$ on a shaker, and then mounted on slides with Aquamount (Thermo Scientific) for microscopy.

\section{Sholl analysis}

Sholl analyses were made using the Linear Sholl Method Plugin for ImageJ. At least 40 microglia were analyzed for each region, with Sholl shells established in $5 \mu \mathrm{m}$ intervals, $10 \mu \mathrm{m}$ from the soma center to the longest observed process length. Briefly, representative images from at least three $40 \mu \mathrm{m}$ sections of a given brain region were used in $\mathrm{n}=3$ mice for each group. Slices were stained 1:300 with Goat-anti-Ibal (Abcam) followed by secondary staining at 1:1000 in Chicken-anti-Goat antibody conjugated to Alexa Fluor 647 (Invitrogen). Z stacks were obtained using a Leica SP8 
Appendix: Methyl-CpG binding protein 2 regulates microglia and macrophage

gene expression in response to inflammatory stimuli

confocal system to profile entire microglia in the $\mathrm{Z}$ direction. $\mathrm{Z}$ planes were taken every $\mu \mathrm{m}$, with max projections utilized for Sholl analysis and detection of soma size.

\section{Quantification of meningeal macrophages}

After staining (see Immunohistochemistry supplemental methods section) $1.35 \mathrm{~mm}^{2}$ images of meninges stained with CD163, F4/80, CD31, and DAPI were taken with a Leica SP8 confocal microscope and blinded for quantification. The total number of $\mathrm{F} 4 / 80^{+} \mathrm{CD} 163^{+}$and $\mathrm{F} 4 / 80^{+} \mathrm{CD} 163^{-}$macrophages were counted per $1.35 \mathrm{~mm}^{2}$ area and used for analysis.

\section{Intestine preparation for flow cytometry}

Mice were perfused with heparinized PBS and the small intestine was removed (duodenum, jejunum and ileum), and then slit along the entire length longitudinally to expose the interior. Intestines were washed 3 times in a petri dish containing HBSS $+5 \%$ FBS $+2 \mathrm{mM}$ EDTA to remove fecal contents. Intestines were then placed in $20 \mathrm{ml} \mathrm{HBSS}$ $+5 \% \mathrm{FBS}+2 \mathrm{mM}$ EDTA at $37^{\circ} \mathrm{C}$ on a shaker at $250 \mathrm{RPM}$ for $20 \mathrm{~m}$, two times. Small intestines were drained through a nylon mesh and finely minced, then placed in $20 \mathrm{ml}$ HBSS $+1.5 \mathrm{mg} / \mathrm{ml}$ collagenase VIII (Sigma) $+40 \mathrm{u} / \mathrm{ml}$ DNAse-I (Sigma) for $15 \mathrm{~m}$ on a shaker at 200 RPM. The resulting suspensions were vortexed for 20 seconds and then passed through a $70 \mu \mathrm{m}$ cell strainer into a $50 \mathrm{ml}$ conical tube. The conical tube was then filled to the top with $\mathrm{HBSS}+5 \% \mathrm{FBS}+2 \mathrm{mM}$ EDTA and centrifuged at $4^{\circ} \mathrm{C}, 425 \mathrm{RCF}$, for $5 \mathrm{~m}$. The supernatant was decanted and the pellet was resuspended in HBSS $+5 \%$ FBS $+2 \mathrm{mM}$ EDTA. Resuspended pellets were centrifuged at $4^{\circ} \mathrm{C}, 425 \mathrm{RCF}$, for $5 \mathrm{~m}$. 
Appendix: Methyl-CpG binding protein 2 regulates microglia and macrophage

gene expression in response to inflammatory stimuli

The supernatant was decanted and the pellet was resuspended in flow cytometry buffer (0.01 M PBS, $1 \%$ BSA, 2 mM EDTA, $0.1 \%$ sodium azide).

\section{Peritoneal macrophage collection for RNA-seq}

Mice were euthanized via $\mathrm{CO}_{2}$ and the skin over the peritoneum was removed. The peritoneal cavity was filled with sterile $0.01 \mathrm{M}$ PBS containing $2 \%$ FBS, and immediately clamped shut with forceps. Filled peritoneal cavities were manually agitated for $1 \mathrm{~m}$, followed by collection of peritoneal lavage fluid via syringe. Cells were then pelleted, and depleted of non-macrophages using biotin-anti-CD3, biotin-anti-B220, and biotinanti-Ly6g antibodies (eBioscience) followed by incubation with anti-biotin magnetic beads (Miltenyi) and negative depletion via AutoMACS. Flow cytometry of AutoMACS sorted cells isolated by this method and gated on singlet events, excluding debris, were

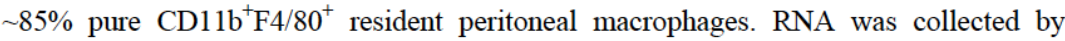
RNeasy mini kit (Qiagen).

\section{Processing and analysis of blood samples}

After euthanasia blood was collected via the retinal artery after removal of the eye. For cell counts, a drop of blood was collected in a heparinized tube followed immediately by counting of a $2 \mu \mathrm{l}$ aliquot diluted in $38 \mu \mathrm{l}$ of an acridine orange and propidium iodide solution to label and enumerate live nucleated cells (leukocytes) on a Nexcelom Cellometer Auto 2000. Blood was additionally collected for flow cytometric analysis. For flow cytometry, blood samples were RBC lysed using ACK lysis buffer (5 m incubation followed by wash in $0.01 \mathrm{M}$ PBS). RBC-lysed samples were then labeled for flow 
Appendix: Methyl-CpG binding protein 2 regulates microglia and macrophage

cytometric analysis as described previously. Counts were obtained by calculating the product of the whole blood cell count measured at the time of blood collection and the percentage of each cell population observed by flow cytometry.

\section{Clodronate and DiI liposome injection}

Clodronate and DiI-labeled liposomes were prepared by a reverse phase evaporation technique as previously described (Faustino et al., 2011) with minor modifications (200 $\mathrm{mg} / \mathrm{ml}$ initial clodronate concentration; DOPC:Cholesterol 20:10 mg/ml final lipid concentration). Liposome size adjustment was achieved by differential centrifugation. Unentrapped clodronate was removed by centrifugation in a microfuge and two washes with 0.01M PBS. Wild type and Mecp2-null littermates, 6-7 weeks old, were injected via tail vein with $100 \mu \mathrm{l}$ clodronate containing liposomes diluted with sterile saline to $200 \mu \mathrm{l}$ total volume. After $48 \mathrm{~h}$, mice were injected via tail vein with $100 \mu \mathrm{l}$ DiI liposomes diluted with sterile saline to a $200 \mu \mathrm{l}$ total volume. Mice were sacrificed on day 5 postclodronate liposome injection and analyzed for monocyte reconstitution and maturation into Ly $6 \mathrm{c}^{\text {lo }}$ resident monocytes.

\section{Chromatin immunoprecipitation DNA sequencing (ChIP-Seq) analysis}

For ChIP-Seq analysis formalin-fixed cells were processed as described (Litvak et al., 2012). BMMs were cross-linked for $10 \mathrm{~min}$. in $1 \%$ paraformaldehyde, washed, lysed and processed for ChIP using anti-rabbit IgG Dynabeads (Invitrogen) pre-conjugated with antibodies against either HDAC3 (sc-11417) (Santa Cruz Biotechnology) or NCOR2

(PA1-843) (Thermo Scientific). The purified ChIP DNA was prepared for sequencing 
Appendix: Methyl-CpG binding protein 2 regulates microglia and macrophage gene expression in response to inflammatory stimuli

with the Illumina ChIP-Seq Sample Prep kit and processed in according to the manufacturer's protocol. The ChIP-Seq data was aligned to the mouse genome (NCBI37/mm9; July 2007) using the ELAND alignment software (Illumina). Regions where the ChIP signals were enriched relative to the normal rabbit serum (NRS) control were determined as described (Litvak et al., 2012; Ramsey et al., 2010). We used a false discovery rate of less than $1 \%$. Integrated Genome Browser (IGB) (Nicol et al., 2009) was used to display the ChIP-Seq data.

\section{Quantitative real-time PCR}

For measurement of the expression of mRNA transcripts total RNA was collected by TRIzol (Invitrogen) for BMDM and resident peritoneal macrophage assays. RNA was collected by Rneasy mini kit (Qiagen) for qRT-PCR of acutely isolated microglia. RNA was reverse-transcribed and analyzed by real-time PCR with TaqMan Gene Expression assays (Applied Biosystems) using the following TaqMan primers: Cxcl2 (Mm00436450_m1); Cxcl3 (Mm01701838_m1); Cyr61 (Mm00487499_g1); Ddit4 (Mm00512504_g1); Hif3a (Mm00469375_m1); Tnf (Mm00443258_m1); $I 16$ (Mm00446190_m1), Fkbp5 (Mm00487406_m1), Il1b (Mm00434228_m1), Csf3 (Mm00438335_g1), Ptx3 (Mm00477268_ml), Cp (Mm01289313_m1), Saa3 (Mm00441203_ml), Hp (Mm00516884_m1), Ccl3 (Mm00441259_g1), Cxcl1 (Mm04207460_m1), Ccl2 (Mm00441242_m1), Il1a (Mm00439620_m1), and Tgfb1 (Mm01178820_m1). Data were acquired using a ViiA ${ }^{\text {TM }} 7$ Real-Time PCR System (Applied Biosystems) and StepOnePlus ${ }^{\mathrm{TM}}$ Real-Time PCR System (Applied Biosystems) and were normalized to the expression of Eeflal mRNA transcripts (encoding eukaryotic 
Appendix: Methyl-CpG binding protein 2 regulates microglia and macrophage

gene expression in response to inflammatory stimuli

translation elongation factor $1 \alpha 1$ ) in individual samples for analysis performed in the Litvak lab. Studies performed in the Kipnis lab were normalized to Hprt transcripts (Mm01545399_ml) and collected using a CFX384 Real-Time PCR Detection System (Bio-Rad).

\section{Normoxia vs. Hypoxia treated BMDM}

Day 7 L929 derived wild type and Mecp2-null BMDMs were grown in either a normoxic tissue culture incubator at $21 \%-\mathrm{O} 2,5 \%-\mathrm{CO}_{2}$ or a humidified hypoxic chamber at $1 \%-\mathrm{O}_{2}$, 5\%- $\mathrm{CO}_{2}$ (Invivo2 200 Hypoxia workstation Biotrace INC) for $24 \mathrm{~h}$. mRNA was isolated for qPCR analysis.

\section{TNF stimulated BMDM}

Day 7 L929 derived wild type and Mecp2-null BMDMs were grown in either a normoxic tissue culture incubator at $21 \%-\mathrm{O} 2,5 \%-\mathrm{CO}_{2}$. Purified TNF $(10 \mathrm{ng} / \mathrm{ml})(\mathrm{eBioscience})$ was added to the media and incubated for $6 \mathrm{~h}$. mRNA was isolated for qRT-PCR analysis.

\section{Analysis of BMDM proliferation per monocyte}

400,000 bone marrow cells from wild type or Mecp2-null counterparts were cultured with $10 \mathrm{ml}$ media and anti-anti (Invitrogen) and 10ng/ml MCSF (eBioscience) in $10 \mathrm{~cm}$ petri dishes, with a second dose of $20 \mathrm{ng} / \mathrm{ml}$ MCSF in $5 \mathrm{ml}$ media added on day 3 of culture. Cells were grown in DMEM/F12 with $10 \%$ FBS and anti-anti (Invitrogen). Number of macrophages generated per starting monocyte was calculated by multiplying

the percentage of $\mathrm{Ly} 6 \mathrm{c}^{\mathrm{hi}} \mathrm{CCR} 2^{+} \mathrm{CD} 115^{+}$monocytes in the initial bone marrow by the 
Appendix: Methyl-CpG binding protein 2 regulates microglia and macrophage

gene expression in response to inflammatory stimuli

starting number of total bone marrow cells $(400,000)$. At the indicated time points, the number of macrophages generated from a single plate was counted and divided by the starting number of monocytes to arrive at the number of macrophages generated per monocyte.

\section{Peritoneal macrophage collection for intraperitoneal TNF injection experiments}

For intraperitoneal TNF experiments, a protocol optimized for the collection of TNFtreated peritoneal macrophages was used. TNF, carrier free (eBioscience) or an equivalent amount of saline was injected into the peritoneum at a dose of $50 \mu \mathrm{g} / \mathrm{kg}(1 \mu \mathrm{g}$ in a $20 \mathrm{~g}$ mouse). After $6 \mathrm{~h}$, mice were euthanized via $\mathrm{CO}_{2}$ and the skin over the peritoneum was removed. The peritoneal cavity was filled with sterile $0.01 \mathrm{M}$ PBS containing $2 \mathrm{mM}$ EDTA, and immediately clamped shut with forceps. Filled peritoneal cavities were manually agitated for $1 \mathrm{~m}$, followed by collection of peritoneal lavage fluid via syringe. Cells were then pelleted, and positively selected for $\mathrm{F} 4 / 80^{+}$macrophages using $\mathrm{F} 4 / 80-\mathrm{PE}$ (eBioscience) followed by incubation with anti-PE magnetic beads (Miltenyi) and positive selection via AutoMACS, which resulted in $70-80 \%$ pure $\mathrm{F} 4 / 80+$ peritoneal macrophages as measured by flow cytometry of singlet events, excluding debris. RNA was collected by TRIzol (Invitrogen).

\section{GCSF neutralizing antibody treatment}

Treatment began at 6-7 $\mathrm{w}$ in either Mecp2-null or wild type controls. Injections with either $5 \mathrm{mg} / \mathrm{kg}$ GCSF neutralizing antibody (clone 67604, R\&D Systems), or Rat IgG1 
Appendix: Methyl-CpG binding protein 2 regulates microglia and macrophage gene expression in response to inflammatory stimuli

isotype control (clone 43414, R\&D Systems) were performed every $48 \mathrm{~h}$. For flow cytometric analysis, mice were analyzed one to two weeks after the start of treatment. 
Appendix: Methyl-CpG binding protein 2 regulates microglia and macrophage gene expression in response to inflammatory stimuli

\section{Supplemental References}

Faustino, J.V., Wang, X., Johnson, C.E., Klibanov, A., Derugin, N., Wendland, M.F., and Vexler, Z.S. (2011). Microglial cells contribute to endogenous brain defenses after acute neonatal focal stroke. The Journal of neuroscience : the official journal of the Society for Neuroscience 31, 12992-13001.

Litvak, V., Ratushny, A.V., Lampano, A.E., Schmitz, F., Huang, A.C., Raman, A., Rust, A.G., Bergthaler, A., Aitchison, J.D., and Aderem, A. (2012). A FOXO3-IRF7 gene regulatory circuit limits inflammatory sequelae of antiviral responses. Nature 490, 421-425.

Nicol, J.W., Helt, G.A., Blanchard, S.G., Jr., Raja, A., and Loraine, A.E. (2009). The Integrated Genome Browser: free software for distribution and exploration of genome-scale datasets. Bioinformatics 25, 2730-2731.

Ramsey, S.A., Knijnenburg, T.A., Kennedy, K.A., Zak, D.E., Gilchrist, M., Gold, E.S., Johnson, C.D., Lampano, A.E., Litvak, V., Navarro, G., et al. (2010). Genome-wide histone acetylation data improve prediction of mammalian transcription factor binding sites. Bioinformatics 26, 2071-2075. 


\section{Works cited}

Alvarez-Saavedra, M., Carrasco, L., Sura-Trueba, S., Demarchi Aiello, V., Walz, K., Neto, J.X., and Young, J.I. (2010). Elevated expression of MeCP2 in cardiac and skeletal tissues is detrimental for normal development. Human molecular genetics 19, 2177-2190.

Alvarez-Saavedra, M., Saez, M.A., Kang, D., Zoghbi, H.Y., and Young, J.I. (2007). Cellspecific expression of wild-type MeCP2 in mouse models of Rett syndrome yields insight about pathogenesis. Human molecular genetics 16, 2315-2325.

American Psychiatric Association., and American Psychiatric Association. DSM-5 Task Force. (2013). Diagnostic and statistical manual of mental disorders : DSM-5, 5th edn (Washington, D.C.: American Psychiatric Association).

Amir, R.E., Van den Veyver, I.B., Wan, M., Tran, C.Q., Francke, U., and Zoghbi, H.Y. (1999). Rett syndrome is caused by mutations in X-linked MECP2, encoding methyl-CpG-binding protein 2. Nature genetics 23, 185-188.

Armulik, A., Genove, G., and Betsholtz, C. (2011). Pericytes: developmental, physiological, and pathological perspectives, problems, and promises. Developmental cell 21, 193-215.

Asaka, Y., Jugloff, D.G., Zhang, L., Eubanks, J.H., and Fitzsimonds, R.M. (2006). Hippocampal synaptic plasticity is impaired in the Mecp2-null mouse model of Rett syndrome. Neurobiology of disease 21, 217-227.

Ballas, N., Lioy, D.T., Grunseich, C., and Mandel, G. (2009). Non-cell autonomous influence of MeCP2-deficient glia on neuronal dendritic morphology. Nature neuroscience $12,311-317$. 
Balmer, D., Arredondo, J., Samaco, R.C., and LaSalle, J.M. (2002). MECP2 mutations in Rett syndrome adversely affect lymphocyte growth, but do not affect imprinted gene expression in blood or brain. Human genetics 110, 545-552.

Balmer, D., Goldstine, J., Rao, Y.M., and LaSalle, J.M. (2003). Elevated methyl-CpGbinding protein 2 expression is acquired during postnatal human brain development and is correlated with alternative polyadenylation. Journal of molecular medicine 81, 61-68.

Bauer, M., Kolsch, U., Kruger, R., Unterwalder, N., Hameister, K., Kaiser, F.M., Vignoli, A., Rossi, R., Botella, M.P., Budisteanu, M., et al. (2015). Infectious and immunologic phenotype of MECP2 duplication syndrome. Journal of clinical immunology 35, 168-181.

Ben-Shachar, S., Chahrour, M., Thaller, C., Shaw, C.A., and Zoghbi, H.Y. (2009). Mouse models of MeCP2 disorders share gene expression changes in the cerebellum and hypothalamus. Human molecular genetics 18, 2431-2442.

Bhatnagar, S., Zhu, X., Ou, J., Lin, L., Chamberlain, L., Zhu, L.J., Wajapeyee, N., and Green, M.R. (2014). Genetic and pharmacological reactivation of the mammalian inactive X chromosome. Proceedings of the National Academy of Sciences of the United States of America 111, 12591-12598.

Bialas, A.R., and Stevens, B. (2013). TGF-beta signaling regulates neuronal C1q expression and developmental synaptic refinement. Nature neuroscience 16, 1773-1782.

Biedermann, B.C. (2001). Vascular endothelium: checkpoint for inflammation and immunity. News in physiological sciences : an international journal of physiology produced jointly by the International Union of Physiological Sciences and the American Physiological Society 16, 84-88. 
Blue, M.E., Boskey, A.L., Doty, S.B., Fedarko, N.S., Hossain, M.A., and Shapiro, J.R. (2015). Osteoblast function and bone histomorphometry in a murine model of Rett syndrome. Bone 76, 23-30.

Bodda, C., Tantra, M., Mollajew, R., Arunachalam, J.P., Laccone, F.A., Can, K., Rosenberger, A., Mironov, S.L., Ehrenreich, H., and Mannan, A.U. (2013). Mild overexpression of Mecp2 in mice causes a higher susceptibility toward seizures. The American journal of pathology 183, 195-210.

Boyes, J., and Bird, A. (1991). DNA methylation inhibits transcription indirectly via a methyl-CpG binding protein. Cell 64, 1123-1134.

Boyes, J., and Bird, A. (1992). Repression of genes by DNA methylation depends on $\mathrm{CpG}$ density and promoter strength: evidence for involvement of a methyl-CpG binding protein. The EMBO journal 11, 327-333.

Braun, S., Kottwitz, D., and Nuber, U.A. (2012). Pharmacological interference with the glucocorticoid system influences symptoms and lifespan in a mouse model of Rett syndrome. Human molecular genetics 21, 1673-1680.

Buchovecky, C.M., Turley, S.D., Brown, H.M., Kyle, S.M., McDonald, J.G., Liu, B., Pieper, A.A., Huang, W., Katz, D.M., Russell, D.W., et al. (2013). A suppressor screen in Mecp2 mutant mice implicates cholesterol metabolism in Rett syndrome. Nature genetics 45, 1013-1020.

Butcher, E.C., and Picker, L.J. (1996). Lymphocyte homing and homeostasis. Science $272,60-66$.

Butovsky, O., Jedrychowski, M.P., Moore, C.S., Cialic, R., Lanser, A.J., Gabriely, G., Koeglsperger, T., Dake, B., Wu, P.M., Doykan, C.E., et al. (2014). Identification of a unique TGF-beta-dependent molecular and functional signature in microglia. Nature neuroscience 17, 131-143. 
Butovsky, O., Ziv, Y., Schwartz, A., Landa, G., Talpalar, A.E., Pluchino, S., Martino, G., and Schwartz, M. (2006). Microglia activated by IL-4 or IFN-gamma differentially induce neurogenesis and oligodendrogenesis from adult stem/progenitor cells. Molecular and cellular neurosciences 31, 149-160.

Carmona, F.D., Cenit, M.C., Diaz-Gallo, L.M., Broen, J.C., Simeon, C.P., Carreira, P.E., Callejas-Rubio, J.L., Fonollosa, V., Lopez-Longo, F.J., Gonzalez-Gay, M.A., et al. (2013). New insight on the Xq28 association with systemic sclerosis. Annals of the rheumatic diseases $72,2032-2038$.

Castro, J., Garcia, R.I., Kwok, S., Banerjee, A., Petravicz, J., Woodson, J., Mellios, N., Tropea, D., and Sur, M. (2014). Functional recovery with recombinant human IGF1 treatment in a mouse model of Rett Syndrome. Proceedings of the National Academy of Sciences of the United States of America 111, 9941-9946.

Chahrour, M., Jung, S.Y., Shaw, C., Zhou, X., Wong, S.T., Qin, J., and Zoghbi, H.Y. (2008). MeCP2, a key contributor to neurological disease, activates and represses transcription. Science 320, 1224-1229.

Chahrour, M., and Zoghbi, H.Y. (2007). The story of Rett syndrome: from clinic to neurobiology. Neuron 56, 422-437.

Chao, H.T., Chen, H., Samaco, R.C., Xue, M., Chahrour, M., Yoo, J., Neul, J.L., Gong, S., Lu, H.C., Heintz, N., et al. (2010). Dysfunction in GABA signalling mediates autism-like stereotypies and Rett syndrome phenotypes. Nature 468, 263-269.

Chao, H.T., Zoghbi, H.Y., and Rosenmund, C. (2007). MeCP2 controls excitatory synaptic strength by regulating glutamatergic synapse number. Neuron 56, 58-65.

Chen, R.Z., Akbarian, S., Tudor, M., and Jaenisch, R. (2001). Deficiency of methyl-CpG binding protein-2 in CNS neurons results in a Rett-like phenotype in mice. Nature genetics $27,327-331$. 
Christodoulou, J., Grimm, A., Maher, T., and Bennetts, B. (2003). RettBASE: The IRSA MECP2 variation database-a new mutation database in evolution. Human mutation $21,466-472$.

Clausen, B.E., Burkhardt, C., Reith, W., Renkawitz, R., and Forster, I. (1999). Conditional gene targeting in macrophages and granulocytes using LysMcre mice. Transgenic research $8,265-277$.

Cobb, B.L., Fei, Y., Jonsson, R., Bolstad, A.I., Brun, J.G., Rischmueller, M., Lester, S.E., Witte, T., Illei, G., Brennan, M., et al. (2010). Genetic association between methyl-CpG binding protein 2 (MECP2) and primary Sjogren's syndrome. Annals of the rheumatic diseases $69,1731-1732$.

Collins, A.L., Levenson, J.M., Vilaythong, A.P., Richman, R., Armstrong, D.L., Noebels, J.L., David Sweatt, J., and Zoghbi, H.Y. (2004). Mild overexpression of MeCP2 causes a progressive neurological disorder in mice. Human molecular genetics 13, 2679-2689.

Conti, V., Gandaglia, A., Galli, F., Tirone, M., Bellini, E., Campana, L., KilstrupNielsen, C., Rovere-Querini, P., Brunelli, S., and Landsberger, N. (2015). MeCP2 Affects Skeletal Muscle Growth and Morphology through Non Cell-Autonomous Mechanisms. PloS one 10, e0130183.

Cook-Mills, J.M., and Deem, T.L. (2005). Active participation of endothelial cells in inflammation. Journal of leukocyte biology 77, 487-495.

Coutinho, A.E., and Chapman, K.E. (2011). The anti-inflammatory and immunosuppressive effects of glucocorticoids, recent developments and mechanistic insights. Molecular and cellular endocrinology 335, 2-13.

Cronk, J.C., Derecki, N.C., Ji, E., Xu, Y., Lampano, A.E., Smirnov, I., Baker, W., Norris, G.T., Marin, I., Coddington, N., et al. (2015a). Methyl-CpG Binding Protein 2 
Regulates Microglia and Macrophage Gene Expression in Response to Inflammatory Stimuli. Immunity 42, 679-691.

Cronk, J.C., Derecki, N.C., Litvak, V., and Kipnis, J. (2015b). Unexpected cellular players in Rett syndrome pathology. Neurobiology of disease.

Cronk, J.C., and Kipnis, J. (2013). Microglia - the brain's busy bees. F1000prime reports 5,53 .

Cuddapah, V.A., Pillai, R.B., Shekar, K.V., Lane, J.B., Motil, K.J., Skinner, S.A., Tarquinio, D.C., Glaze, D.G., McGwin, G., Kaufmann, W.E., et al. (2014). Methyl-CpG-binding protein 2 (MECP2) mutation type is associated with disease severity in Rett syndrome. Journal of medical genetics 51, 152-158.

Cunningham, C.L., Martinez-Cerdeno, V., and Noctor, S.C. (2013). Microglia regulate the number of neural precursor cells in the developing cerebral cortex. The Journal of neuroscience : the official journal of the Society for Neuroscience 33, 4216-4233.

Danese, S., Dejana, E., and Fiocchi, C. (2007). Immune regulation by microvascular endothelial cells: directing innate and adaptive immunity, coagulation, and inflammation. Journal of immunology 178, 6017-6022.

Dani, V.S., Chang, Q., Maffei, A., Turrigiano, G.G., Jaenisch, R., and Nelson, S.B. (2005). Reduced cortical activity due to a shift in the balance between excitation and inhibition in a mouse model of Rett syndrome. Proceedings of the National Academy of Sciences of the United States of America 102, 12560-12565.

Davies, L.C., Jenkins, S.J., Allen, J.E., and Taylor, P.R. (2013). Tissue-resident macrophages. Nature immunology 14, 986-995. 
De Felice, C., Rossi, M., Leoncini, S., Chisci, G., Signorini, C., Lonetti, G., Vannuccini, L., Spina, D., Ginori, A., Iacona, I., et al. (2014). Inflammatory lung disease in Rett syndrome. Mediators of inflammation 2014, 560120.

Deaton, A.M., and Bird, A. (2011). CpG islands and the regulation of transcription. Genes \& development 25, 1010-1022.

del Gaudio, D., Fang, P., Scaglia, F., Ward, P.A., Craigen, W.J., Glaze, D.G., Neul, J.L., Patel, A., Lee, J.A., Irons, M., et al. (2006). Increased MECP2 gene copy number as the result of genomic duplication in neurodevelopmentally delayed males. Genetics in medicine : official journal of the American College of Medical Genetics 8, 784-792.

Delepine, C., Nectoux, J., Letourneur, F., Baud, V., Chelly, J., Billuart, P., and Bienvenu, T. (2015). Astrocyte Transcriptome from the Mecp2(308)-Truncated Mouse Model of Rett Syndrome. Neuromolecular medicine 17, 353-363.

Derecki, N.C., Cronk, J.C., Lu, Z., Xu, E., Abbott, S.B., Guyenet, P.G., and Kipnis, J. (2012). Wild-type microglia arrest pathology in a mouse model of Rett syndrome. Nature 484, 105-109.

Ebert, D.H., Gabel, H.W., Robinson, N.D., Kastan, N.R., Hu, L.S., Cohen, S., Navarro, A.J., Lyst, M.J., Ekiert, R., Bird, A.P., and Greenberg, M.E. (2013). Activitydependent phosphorylation of $\mathrm{MeCP} 2$ threonine 308 regulates interaction with NCoR. Nature 499, 341-345.

Ellaway, C.J., Sholler, G., Leonard, H., and Christodoulou, J. (1999). Prolonged QT interval in Rett syndrome. Archives of disease in childhood 80, 470-472.

Friez, M.J., Jones, J.R., Clarkson, K., Lubs, H., Abuelo, D., Bier, J.A., Pai, S., Simensen, R., Williams, C., Giampietro, P.F., et al. (2006). Recurrent infections, hypotonia, 
and mental retardation caused by duplication of MECP2 and adjacent region in Xq28. Pediatrics 118, e1687-1695.

Fyffe, S.L., Neul, J.L., Samaco, R.C., Chao, H.T., Ben-Shachar, S., Moretti, P., McGill, B.E., Goulding, E.H., Sullivan, E., Tecott, L.H., and Zoghbi, H.Y. (2008). Deletion of Mecp2 in Sim1-expressing neurons reveals a critical role for MeCP2 in feeding behavior, aggression, and the response to stress. Neuron 59, 947-958.

Garg, S.K., Lioy, D.T., Cheval, H., McGann, J.C., Bissonnette, J.M., Murtha, M.J., Foust, K.D., Kaspar, B.K., Bird, A., and Mandel, G. (2013). Systemic delivery of MeCP2 rescues behavioral and cellular deficits in female mouse models of Rett syndrome. The Journal of neuroscience : the official journal of the Society for Neuroscience 33, 13612-13620.

Gemelli, T., Berton, O., Nelson, E.D., Perrotti, L.I., Jaenisch, R., and Monteggia, L.M. (2006). Postnatal loss of methyl-CpG binding protein 2 in the forebrain is sufficient to mediate behavioral aspects of Rett syndrome in mice. Biological psychiatry 59, 468-476.

Giacometti, E., Luikenhuis, S., Beard, C., and Jaenisch, R. (2007). Partial rescue of $\mathrm{MeCP} 2$ deficiency by postnatal activation of MeCP2. Proceedings of the National Academy of Sciences of the United States of America 104, 1931-1936.

Glaze, D.G. (2005). Neurophysiology of Rett syndrome. Journal of child neurology 20, 740-746.

Gold, W.A., Williamson, S.L., Kaur, S., Hargreaves, I.P., Land, J.M., Pelka, G.J., Tam, P.P., and Christodoulou, J. (2014). Mitochondrial dysfunction in the skeletal muscle of a mouse model of Rett syndrome (RTT): implications for the disease phenotype. Mitochondrion 15, 10-17. 
Goldmann, T., Wieghofer, P., Muller, P.F., Wolf, Y., Varol, D., Yona, S., Brendecke, S.M., Kierdorf, K., Staszewski, O., Datta, M., et al. (2013). A new type of microglia gene targeting shows TAK1 to be pivotal in CNS autoimmune inflammation. Nature neuroscience 16, 1618-1626.

Graham, M.B., Dalton, D.K., Giltinan, D., Braciale, V.L., Stewart, T.A., and Braciale, T.J. (1993). Response to influenza infection in mice with a targeted disruption in the interferon gamma gene. The Journal of experimental medicine 178, 17251732.

Grasshoff, U., Bonin, M., Goehring, I., Ekici, A., Dufke, A., Cremer, K., Wagner, N., Rossier, E., Jauch, A., Walter, M., et al. (2011). De novo MECP2 duplication in two females with random $\mathrm{X}$-inactivation and moderate mental retardation. European journal of human genetics : EJHG 19, 507-512.

Guy, J., Cheval, H., Selfridge, J., and Bird, A. (2011). The role of MeCP2 in the brain. Annual review of cell and developmental biology 27, 631-652.

Guy, J., Gan, J., Selfridge, J., Cobb, S., and Bird, A. (2007). Reversal of neurological defects in a mouse model of Rett syndrome. Science 315, 1143-1147.

Guy, J., Hendrich, B., Holmes, M., Martin, J.E., and Bird, A. (2001). A mouse Mecp2null mutation causes neurological symptoms that mimic Rett syndrome. Nature genetics $27,322-326$.

Hagberg, B. (2002). Clinical manifestations and stages of Rett syndrome. Mental retardation and developmental disabilities research reviews 8, 61-65.

Hagberg, B. (2005). Rett syndrome: long-term clinical follow-up experiences over four decades. Journal of child neurology 20, 722-727. 
Hagberg, B., Aicardi, J., Dias, K., and Ramos, O. (1983). A progressive syndrome of autism, dementia, ataxia, and loss of purposeful hand use in girls: Rett's syndrome: report of 35 cases. Annals of neurology 14, 471-479.

Han, T.U., Cho, S.K., Kim, T., Joo, Y.B., Bae, S.C., and Kang, C. (2013). Association of an activity-enhancing variant of IRAK1 and an MECP2-IRAK1 haplotype with increased susceptibility to rheumatoid arthritis. Arthritis and rheumatism 65, 590598.

Hogquist, K.A., Jameson, S.C., Heath, W.R., Howard, J.L., Bevan, M.J., and Carbone, F.R. (1994). T cell receptor antagonist peptides induce positive selection. Cell 76, 17-27.

Horiuchi, M., Smith, L., Maezawa, I., and Jin, L.W. (2016). CXCR1 ablation ameliorates motor and respiratory dysfunctions and improves survival of a Rett syndrome mouse model. Brain, behavior, and immunity.

Ito-Ishida, A., Ure, K., Chen, H., Swann, J.W., and Zoghbi, H.Y. (2015). Loss of MeCP2 in Parvalbumin-and Somatostatin-Expressing Neurons in Mice Leads to Distinct Rett Syndrome-like Phenotypes. Neuron 88, 651-658.

Jaenisch, R., and Bird, A. (2003). Epigenetic regulation of gene expression: how the genome integrates intrinsic and environmental signals. Nature genetics 33 Suppl, 245-254.

Jan, M.M., Dooley, J.M., and Gordon, K.E. (1999). Male Rett syndrome variant: application of diagnostic criteria. Pediatric neurology 20, 238-240.

Jeyaseelan, S., Manzer, R., Young, S.K., Yamamoto, M., Akira, S., Mason, R.J., and Worthen, G.S. (2005). Induction of CXCL5 during inflammation in the rodent lung involves activation of alveolar epithelium. American journal of respiratory cell and molecular biology 32, 531-539. 
Ji, K., Akgul, G., Wollmuth, L.P., and Tsirka, S.E. (2013). Microglia actively regulate the number of functional synapses. PloS one 8 , e56293.

Jiang, S., Li, C., McRae, G., Lykken, E., Sevilla, J., Liu, S.Q., Wan, Y., and Li, Q.J. (2014). MeCP2 reinforces STAT3 signaling and the generation of effector CD4+ $\mathrm{T}$ cells by promoting miR-124-mediated suppression of SOCS5. Science signaling 7, ra25.

Jin, L.W., Horiuchi, M., Wulff, H., Liu, X.B., Cortopassi, G.A., Erickson, J.D., and Maezawa, I. (2015). Dysregulation of Glutamine Transporter SNAT1 in Rett Syndrome Microglia: A Mechanism for Mitochondrial Dysfunction and Neurotoxicity. The Journal of neuroscience : the official journal of the Society for Neuroscience 35, 2516-2529.

Jones, P.L., Veenstra, G.J., Wade, P.A., Vermaak, D., Kass, S.U., Landsberger, N., Strouboulis, J., and Wolffe, A.P. (1998). Methylated DNA and MeCP2 recruit histone deacetylase to repress transcription. Nature genetics 19, 187-191.

Jung, B.P., Jugloff, D.G., Zhang, G., Logan, R., Brown, S., and Eubanks, J.H. (2003). The expression of methyl $\mathrm{CpG}$ binding factor MeCP2 correlates with cellular differentiation in the developing rat brain and in cultured cells. Journal of neurobiology 55, 86-96.

Jung, S., Aliberti, J., Graemmel, P., Sunshine, M.J., Kreutzberg, G.W., Sher, A., and Littman, D.R. (2000). Analysis of fractalkine receptor CX(3)CR1 function by targeted deletion and green fluorescent protein reporter gene insertion. Molecular and cellular biology 20, 4106-4114.

Kaufman, K.M., Zhao, J., Kelly, J.A., Hughes, T., Adler, A., Sanchez, E., Ojwang, J.O., Langefeld, C.D., Ziegler, J.T., Williams, A.H., et al. (2013). Fine mapping of Xq28: both MECP2 and IRAK1 contribute to risk for systemic lupus 
erythematosus in multiple ancestral groups. Annals of the rheumatic diseases 72 , 437-444.

Kerr, A.M., Armstrong, D.D., Prescott, R.J., Doyle, D., and Kearney, D.L. (1997). Rett syndrome: analysis of deaths in the British survey. European child \& adolescent psychiatry 6 Suppl 1, 71-74.

Khwaja, O.S., Ho, E., Barnes, K.V., O'Leary, H.M., Pereira, L.M., Finkelstein, Y., Nelson, C.A., 3rd, Vogel-Farley, V., DeGregorio, G., Holm, I.A., et al. (2014). Safety, pharmacokinetics, and preliminary assessment of efficacy of mecasermin (recombinant human IGF-1) for the treatment of Rett syndrome. Proceedings of the National Academy of Sciences of the United States of America 111, 45964601.

Kishi, N., and Macklis, J.D. (2004). MECP2 is progressively expressed in post-migratory neurons and is involved in neuronal maturation rather than cell fate decisions. Molecular and cellular neurosciences 27, 306-321.

Koelsch, K.A., Webb, R., Jeffries, M., Dozmorov, M.G., Frank, M.B., Guthridge, J.M., James, J.A., Wren, J.D., and Sawalha, A.H. (2013). Functional characterization of the MECP2/IRAK1 lupus risk haplotype in human T cells and a human MECP2 transgenic mouse. Journal of autoimmunity 41, 168-174.

Kokura, K., Kaul, S.C., Wadhwa, R., Nomura, T., Khan, M.M., Shinagawa, T., Yasukawa, T., Colmenares, C., and Ishii, S. (2001). The Ski protein family is required for MeCP2-mediated transcriptional repression. The Journal of biological chemistry 276, 34115-34121.

Koltsova, E.K., and Ley, K. (2010). The mysterious ways of the chemokine CXCL5. Immunity 33, 7-9. 
Lerner, M.R., and Steitz, J.A. (1979). Antibodies to small nuclear RNAs complexed with proteins are produced by patients with systemic lupus erythematosus. Proceedings of the National Academy of Sciences of the United States of America 76, 54955499.

Lewis, J.D., Meehan, R.R., Henzel, W.J., Maurer-Fogy, I., Jeppesen, P., Klein, F., and Bird, A. (1992). Purification, sequence, and cellular localization of a novel chromosomal protein that binds to methylated DNA. Cell 69, 905-914.

Li, C., Jiang, S., Liu, S.Q., Lykken, E., Zhao, L.T., Sevilla, J., Zhu, B., and Li, Q.J. (2014). MeCP2 enforces Foxp3 expression to promote regulatory $\mathrm{T}$ cells' resilience to inflammation. Proceedings of the National Academy of Sciences of the United States of America 111, E2807-2816.

Lioy, D.T., Garg, S.K., Monaghan, C.E., Raber, J., Foust, K.D., Kaspar, B.K., Hirrlinger, P.G., Kirchhoff, F., Bissonnette, J.M., Ballas, N., and Mandel, G. (2011). A role for glia in the progression of Rett's syndrome. Nature 475, 497-500.

Lombardi, L.M., Baker, S.A., and Zoghbi, H.Y. (2015). MECP2 disorders: from the clinic to mice and back. The Journal of clinical investigation 125, 2914-2923.

Luikenhuis, S., Giacometti, E., Beard, C.F., and Jaenisch, R. (2004). Expression of $\mathrm{MeCP} 2$ in postmitotic neurons rescues Rett syndrome in mice. Proceedings of the National Academy of Sciences of the United States of America 101, 6033-6038.

Lunyak, V.V., Burgess, R., Prefontaine, G.G., Nelson, C., Sze, S.H., Chenoweth, J., Schwartz, P., Pevzner, P.A., Glass, C., Mandel, G., and Rosenfeld, M.G. (2002). Corepressor-dependent silencing of chromosomal regions encoding neuronal genes. Science 298, 1747-1752.

Lyst, M.J., Ekiert, R., Ebert, D.H., Merusi, C., Nowak, J., Selfridge, J., Guy, J., Kastan, N.R., Robinson, N.D., de Lima Alves, F., et al. (2013). Rett syndrome mutations 
abolish the interaction of MeCP2 with the NCoR/SMRT co-repressor. Nature neuroscience $16,898-902$.

Macpherson, A.J., McCoy, K.D., Johansen, F.E., and Brandtzaeg, P. (2008). The immune geography of IgA induction and function. Mucosal immunology 1, 11-22.

Maezawa, I., and Jin, L.W. (2010). Rett syndrome microglia damage dendrites and synapses by the elevated release of glutamate. The Journal of neuroscience : the official journal of the Society for Neuroscience 30, 5346-5356.

Maezawa, I., Swanberg, S., Harvey, D., LaSalle, J.M., and Jin, L.W. (2009). Rett syndrome astrocytes are abnormal and spread MeCP2 deficiency through gap junctions. The Journal of neuroscience : the official journal of the Society for Neuroscience 29, 5051-5061.

Makrythanasis, P., Moix, I., Gimelli, S., Fluss, J., Aliferis, K., Antonarakis, S.E., Morris, M.A., Bena, F., and Bottani, A. (2010). De novo duplication of MECP2 in a girl with mental retardation and no obvious dysmorphic features. Clinical genetics 78 , 175-180.

Mayo, S., Monfort, S., Rosello, M., Orellana, C., Oltra, S., Armstrong, J., Catala, V., and Martinez, F. (2011). De novo interstitial triplication of MECP2 in a girl with neurodevelopmental disorder and random $\mathrm{X}$ chromosome inactivation. Cytogenetic and genome research 135, 93-101.

McGraw, C.M., Samaco, R.C., and Zoghbi, H.Y. (2011). Adult neural function requires MeCP2. Science 333, 186.

Meehan, R.R., Lewis, J.D., and Bird, A.P. (1992). Characterization of MeCP2, a vertebrate DNA binding protein with affinity for methylated DNA. Nucleic acids research 20, 5085-5092. 
Mei, J., Liu, Y., Dai, N., Favara, M., Greene, T., Jeyaseelan, S., Poncz, M., Lee, J.S., and Worthen, G.S. (2010). CXCL5 regulates chemokine scavenging and pulmonary host defense to bacterial infection. Immunity 33, 106-117.

Meins, M., Lehmann, J., Gerresheim, F., Herchenbach, J., Hagedorn, M., Hameister, K., and Epplen, J.T. (2005). Submicroscopic duplication in Xq28 causes increased expression of the MECP2 gene in a boy with severe mental retardation and features of Rett syndrome. Journal of medical genetics 42 , e12.

Mellen, M., Ayata, P., Dewell, S., Kriaucionis, S., and Heintz, N. (2012). MeCP2 binds to $5 \mathrm{hmC}$ enriched within active genes and accessible chromatin in the nervous system. Cell 151, 1417-1430.

Mendez-Ferrer, S., Michurina, T.V., Ferraro, F., Mazloom, A.R., Macarthur, B.D., Lira, S.A., Scadden, D.T., Ma'ayan, A., Enikolopov, G.N., and Frenette, P.S. (2010). Mesenchymal and haematopoietic stem cells form a unique bone marrow niche. Nature 466, 829-834.

Moretti, P., Levenson, J.M., Battaglia, F., Atkinson, R., Teague, R., Antalffy, B., Armstrong, D., Arancio, O., Sweatt, J.D., and Zoghbi, H.Y. (2006). Learning and memory and synaptic plasticity are impaired in a mouse model of Rett syndrome. The Journal of neuroscience : the official journal of the Society for Neuroscience 26, 319-327.

Motil, K.J., Barrish, J.O., Neul, J.L., and Glaze, D.G. (2014). Low bone mineral mass is associated with decreased bone formation and diet in girls with Rett syndrome. Journal of pediatric gastroenterology and nutrition 59, 386-392.

Motil, K.J., Caeg, E., Barrish, J.O., Geerts, S., Lane, J.B., Percy, A.K., Annese, F., McNair, L., Skinner, S.A., Lee, H.S., et al. (2012). Gastrointestinal and nutritional problems occur frequently throughout life in girls and women with Rett syndrome. Journal of pediatric gastroenterology and nutrition 55, 292-298. 
Mullaney, B.C., Johnston, M.V., and Blue, M.E. (2004). Developmental expression of methyl-CpG binding protein 2 is dynamically regulated in the rodent brain. Neuroscience 123, 939-949.

Nan, X., Ng, H.H., Johnson, C.A., Laherty, C.D., Turner, B.M., Eisenman, R.N., and Bird, A. (1998). Transcriptional repression by the methyl-CpG-binding protein MeCP2 involves a histone deacetylase complex. Nature 393, 386-389.

Nelson, E.D., Kavalali, E.T., and Monteggia, L.M. (2006). MeCP2-dependent transcriptional repression regulates excitatory neurotransmission. Current biology : CB 16, 710-716.

Neul, J.L., Kaufmann, W.E., Glaze, D.G., Christodoulou, J., Clarke, A.J., Bahi-Buisson, N., Leonard, H., Bailey, M.E., Schanen, N.C., Zappella, M., et al. (2010). Rett syndrome: revised diagnostic criteria and nomenclature. Annals of neurology 68 , 944-950.

Neul, J.L., Lane, J.B., Lee, H.S., Geerts, S., Barrish, J.O., Annese, F., Baggett, L.M., Barnes, K., Skinner, S.A., Motil, K.J., et al. (2014). Developmental delay in Rett syndrome: data from the natural history study. Journal of neurodevelopmental disorders 6, 20.

Nguyen, M.V., Felice, C.A., Du, F., Covey, M.V., Robinson, J.K., Mandel, G., and Ballas, N. (2013). Oligodendrocyte lineage cells contribute unique features to Rett syndrome neuropathology. The Journal of neuroscience : the official journal of the Society for Neuroscience 33, 18764-18774.

Nouailles, G., Dorhoi, A., Koch, M., Zerrahn, J., Weiner, J., 3rd, Fae, K.C., Arrey, F., Kuhlmann, S., Bandermann, S., Loewe, D., et al. (2014). CXCL5-secreting pulmonary epithelial cells drive destructive neutrophilic inflammation in tuberculosis. The Journal of clinical investigation 124, 1268-1282. 
Nuber, U.A., Kriaucionis, S., Roloff, T.C., Guy, J., Selfridge, J., Steinhoff, C., Schulz, R., Lipkowitz, B., Ropers, H.H., Holmes, M.C., and Bird, A. (2005). Up-regulation of glucocorticoid-regulated genes in a mouse model of Rett syndrome. Human molecular genetics 14, 2247-2256.

O'Connor, R.D., Zayzafoon, M., Farach-Carson, M.C., and Schanen, N.C. (2009). Mecp2 deficiency decreases bone formation and reduces bone volume in a rodent model of Rett syndrome. Bone 45, 346-356.

O'Driscoll, C., Kaufmann, W.E., and Bressler, J. (2013a). Relationship between Mecp2 and NFkappab signaling during neural differentiation of P19 cells. Brain research $1490,35-42$.

O'Driscoll, C.M., Kaufmann, W.E., and Bressler, J.P. (2013b). MeCP2 deficiency enhances glutamate release through NF-kappaB signaling in myeloid derived cells. Journal of neuroimmunology 265, 61-67.

O'Driscoll, C.M., Lima, M.P., Kaufmann, W.E., and Bressler, J.P. (2015). Methyl CpG binding protein 2 deficiency enhances expression of inflammatory cytokines by sustaining NF-kappaB signaling in myeloid derived cells. Journal of neuroimmunology 283, 23-29.

Okabe, Y., Takahashi, T., Mitsumasu, C., Kosai, K., Tanaka, E., and Matsuishi, T. (2012). Alterations of gene expression and glutamate clearance in astrocytes derived from an MeCP2-null mouse model of Rett syndrome. PloS one 7, e35354.

Paolicelli, R.C., Bolasco, G., Pagani, F., Maggi, L., Scianni, M., Panzanelli, P., Giustetto, M., Ferreira, T.A., Guiducci, E., Dumas, L., et al. (2011). Synaptic pruning by microglia is necessary for normal brain development. Science 333, 1456-1458.

Parkhurst, C.N., Yang, G., Ninan, I., Savas, J.N., Yates, J.R., 3rd, Lafaille, J.J., Hempstead, B.L., Littman, D.R., and Gan, W.B. (2013). Microglia promote 
learning-dependent synapse formation through brain-derived neurotrophic factor. Cell 155, 1596-1609.

Pini, G., Scusa, M.F., Congiu, L., Benincasa, A., Morescalchi, P., Bottiglioni, I., Di Marco, P., Borelli, P., Bonuccelli, U., Della-Chiesa, A., et al. (2012). IGF1 as a Potential Treatment for Rett Syndrome: Safety Assessment in Six Rett Patients. Autism research and treatment 2012, 679801.

Prinz, M., and Priller, J. (2014). Microglia and brain macrophages in the molecular age: from origin to neuropsychiatric disease. Nature reviews. Neuroscience 15, 300312.

Ramocki, M.B., Peters, S.U., Tavyev, Y.J., Zhang, F., Carvalho, C.M., Schaaf, C.P., Richman, R., Fang, P., Glaze, D.G., Lupski, J.R., and Zoghbi, H.Y. (2009). Autism and other neuropsychiatric symptoms are prevalent in individuals with MeCP2 duplication syndrome. Annals of neurology 66, 771-782.

Ramocki, M.B., Tavyev, Y.J., and Peters, S.U. (2010). The MECP2 duplication syndrome. American journal of medical genetics. Part A 152A, 1079-1088.

Rett, A. (1966). [On a unusual brain atrophy syndrome in hyperammonemia in childhood]. Wiener medizinische Wochenschrift 116, 723-726.

Roze, E., Cochen, V., Sangla, S., Bienvenu, T., Roubergue, A., Leu-Semenescu, S., and Vidaihet, M. (2007). Rett syndrome: an overlooked diagnosis in women with stereotypic hand movements, psychomotor retardation, Parkinsonism, and dystonia? Movement disorders : official journal of the Movement Disorder Society $22,387-389$.

Sakaguchi, S., Miyara, M., Costantino, C.M., and Hafler, D.A. (2010). FOXP3+ regulatory $\mathrm{T}$ cells in the human immune system. Nature reviews. Immunology 10, 490-500. 
Samaco, R.C., Mandel-Brehm, C., Chao, H.T., Ward, C.S., Fyffe-Maricich, S.L., Ren, J., Hyland, K., Thaller, C., Maricich, S.M., Humphreys, P., et al. (2009). Loss of $\mathrm{MeCP} 2$ in aminergic neurons causes cell-autonomous defects in neurotransmitter synthesis and specific behavioral abnormalities. Proceedings of the National Academy of Sciences of the United States of America 106, 21966-21971.

Samaco, R.C., Mandel-Brehm, C., McGraw, C.M., Shaw, C.A., McGill, B.E., and Zoghbi, H.Y. (2012). Crh and Oprm1 mediate anxiety-related behavior and social approach in a mouse model of MECP2 duplication syndrome. Nature genetics 44, 206-211.

Sanlaville, D., Prieur, M., de Blois, M.C., Genevieve, D., Lapierre, J.M., Ozilou, C., Picq, M., Gosset, P., Morichon-Delvallez, N., Munnich, A., et al. (2005). Functional disomy of the Xq28 chromosome region. European journal of human genetics : EJHG 13, 579-585.

Sawalha, A.H., Webb, R., Han, S., Kelly, J.A., Kaufman, K.M., Kimberly, R.P., AlarconRiquelme, M.E., James, J.A., Vyse, T.J., Gilkeson, G.S., et al. (2008). Common variants within MECP2 confer risk of systemic lupus erythematosus. PloS one 3, e1727.

Schafer, D.P., Lehrman, E.K., Kautzman, A.G., Koyama, R., Mardinly, A.R., Yamasaki, R., Ransohoff, R.M., Greenberg, M.E., Barres, B.A., and Stevens, B. (2012). Microglia sculpt postnatal neural circuits in an activity and complementdependent manner. Neuron 74, 691-705.

Sekul, E.A., Moak, J.P., Schultz, R.J., Glaze, D.G., Dunn, J.K., and Percy, A.K. (1994). Electrocardiographic findings in Rett syndrome: an explanation for sudden death? The Journal of pediatrics $125,80-82$. 
Shahbazian, M.D., Antalffy, B., Armstrong, D.L., and Zoghbi, H.Y. (2002). Insight into Rett syndrome: MeCP2 levels display tissue- and cell-specific differences and correlate with neuronal maturation. Human molecular genetics 11, 115-124.

Sharma, K., Singh, J., Pillai, P.P., and Frost, E.E. (2015). Involvement of MeCP2 in Regulation of Myelin-Related Gene Expression in Cultured Rat Oligodendrocytes. Journal of molecular neuroscience : MN 57, 176-184.

Shimada, S., Okamoto, N., Hirasawa, K., Yoshii, K., Tani, Y., Sugawara, M., Shimojima, K., Osawa, M., and Yamamoto, T. (2013a). Clinical manifestations of Xq28 functional disomy involving MECP2 in one female and two male patients. American journal of medical genetics. Part A 161A, 1779-1785.

Shimada, S., Okamoto, N., Ito, M., Arai, Y., Momosaki, K., Togawa, M., Maegaki, Y., Sugawara, M., Shimojima, K., Osawa, M., and Yamamoto, T. (2013b). MECP2 duplication syndrome in both genders. Brain \& development 35, 411-419.

Sierra, A., Encinas, J.M., Deudero, J.J., Chancey, J.H., Enikolopov, G., OverstreetWadiche, L.S., Tsirka, S.E., and Maletic-Savatic, M. (2010). Microglia shape adult hippocampal neurogenesis through apoptosis-coupled phagocytosis. Cell stem cell 7, 483-495.

Skene, P.J., Illingworth, R.S., Webb, S., Kerr, A.R., James, K.D., Turner, D.J., Andrews, R., and Bird, A.P. (2010). Neuronal MeCP2 is expressed at near histone-octamer levels and globally alters the chromatin state. Molecular cell 37, 457-468.

Smyk, M., Obersztyn, E., Nowakowska, B., Nawara, M., Cheung, S.W., Mazurczak, T., Stankiewicz, P., and Bocian, E. (2008). Different-sized duplications of Xq28, including MECP2, in three males with mental retardation, absent or delayed speech, and recurrent infections. American journal of medical genetics. Part B, Neuropsychiatric genetics : the official publication of the International Society of Psychiatric Genetics 147B, 799-806. 
Song, C., Feodorova, Y., Guy, J., Peichl, L., Jost, K.L., Kimura, H., Cardoso, M.C., Bird, A., Leonhardt, H., Joffe, B., and Solovei, I. (2014). DNA methylation reader MECP2: cell type- and differentiation stage-specific protein distribution. Epigenetics \& chromatin 7, 17.

Steffenburg, U., Hagberg, G., and Hagberg, B. (2001). Epilepsy in a representative series of Rett syndrome. Acta paediatrica 90, 34-39.

Stellwagen, D., and Malenka, R.C. (2006). Synaptic scaling mediated by glial TNFalpha. Nature 440, 1054-1059.

Suzuki, M., Yamada, T., Kihara-Negishi, F., Sakurai, T., and Oikawa, T. (2003). Direct association between PU.1 and MeCP2 that recruits mSin3A-HDAC complex for PU.1-mediated transcriptional repression. Oncogene 22, 8688-8698.

Sztainberg, Y., Chen, H.M., Swann, J.W., Hao, S., Tang, B., Wu, Z., Tang, J., Wan, Y.W., Liu, Z., Rigo, F., and Zoghbi, H.Y. (2015). Reversal of phenotypes in MECP2 duplication mice using genetic rescue or antisense oligonucleotides. Nature 528, 123-126.

Teitelbaum, S.L. (2000). Bone resorption by osteoclasts. Science 289, 1504-1508.

Trapp, B.D., Wujek, J.R., Criste, G.A., Jalabi, W., Yin, X., Kidd, G.J., Stohlman, S., and Ransohoff, R. (2007). Evidence for synaptic stripping by cortical microglia. Glia $55,360-368$.

Tropea, D., Giacometti, E., Wilson, N.R., Beard, C., McCurry, C., Fu, D.D., Flannery, R., Jaenisch, R., and Sur, M. (2009). Partial reversal of Rett Syndrome-like symptoms in MeCP2 mutant mice. Proceedings of the National Academy of Sciences of the United States of America 106, 2029-2034.

Tudor, M., Akbarian, S., Chen, R.Z., and Jaenisch, R. (2002). Transcriptional profiling of a mouse model for Rett syndrome reveals subtle transcriptional changes in the 
brain. Proceedings of the National Academy of Sciences of the United States of America 99, 15536-15541.

Ueno, M., Fujita, Y., Tanaka, T., Nakamura, Y., Kikuta, J., Ishii, M., and Yamashita, T. (2013). Layer V cortical neurons require microglial support for survival during postnatal development. Nature neuroscience 16, 543-551.

Van Esch, H., Bauters, M., Ignatius, J., Jansen, M., Raynaud, M., Hollanders, K., Lugtenberg, D., Bienvenu, T., Jensen, L.R., Gecz, J., et al. (2005). Duplication of the MECP2 region is a frequent cause of severe mental retardation and progressive neurological symptoms in males. American journal of human genetics $77,442-453$.

Waisman, A., Ginhoux, F., Greter, M., and Bruttger, J. (2015). Homeostasis of Microglia in the Adult Brain: Review of Novel Microglia Depletion Systems. Trends in immunology 36, 625-636.

Webb, R., Wren, J.D., Jeffries, M., Kelly, J.A., Kaufman, K.M., Tang, Y., Frank, M.B., Merrill, J., Kimberly, R.P., Edberg, J.C., et al. (2009). Variants within MECP2, a key transcription regulator, are associated with increased susceptibility to lupus and differential gene expression in patients with systemic lupus erythematosus. Arthritis and rheumatism 60, 1076-1084.

Weir, E.K., and Archer, S.L. (1995). The mechanism of acute hypoxic pulmonary vasoconstriction: the tale of two channels. FASEB journal : official publication of the Federation of American Societies for Experimental Biology 9, 183-189.

Weng, S.M., McLeod, F., Bailey, M.E., and Cobb, S.R. (2011). Synaptic plasticity deficits in an experimental model of rett syndrome: long-term potentiation saturation and its pharmacological reversal. Neuroscience 180, 314-321. 
Wheeler, M.A., Heffner, D.L., Kim, S., Espy, S.M., Spano, A.J., Cleland, C.L., and Deppmann, C.D. (2014). TNF-alpha/TNFR1 signaling is required for the development and function of primary nociceptors. Neuron $82,587-602$.

Williams, E.C., Zhong, X., Mohamed, A., Li, R., Liu, Y., Dong, Q., Ananiev, G.E., Mok, J.C., Lin, B.R., Lu, J., et al. (2014). Mutant astrocytes differentiated from Rett syndrome patients-specific iPSCs have adverse effects on wild-type neurons. Human molecular genetics 23, 2968-2980.

Wu, Y., Borde, M., Heissmeyer, V., Feuerer, M., Lapan, A.D., Stroud, J.C., Bates, D.L., Guo, L., Han, A., Ziegler, S.F., et al. (2006). FOXP3 controls regulatory T cell function through cooperation with NFAT. Cell 126, 375-387.

Yang, T., Ramocki, M.B., Neul, J.L., Lu, W., Roberts, L., Knight, J., Ward, C.S., Zoghbi, H.Y., Kheradmand, F., and Corry, D.B. (2012). Overexpression of methyl-CpG binding protein 2 impairs $\mathrm{T}(\mathrm{H}) 1$ responses. Science translational medicine 4 , $163 \mathrm{ra} 158$.

Yasui, D.H., Xu, H., Dunaway, K.W., Lasalle, J.M., Jin, L.W., and Maezawa, I. (2013). $\mathrm{MeCP} 2$ modulates gene expression pathways in astrocytes. Molecular autism 4, 3 .

Yona, S., Kim, K.W., Wolf, Y., Mildner, A., Varol, D., Breker, M., Strauss-Ayali, D., Viukov, S., Guilliams, M., Misharin, A., et al. (2013). Fate mapping reveals origins and dynamics of monocytes and tissue macrophages under homeostasis. Immunity 38, 79-91.

Yu, F., Thiesen, J., and Stratling, W.H. (2000). Histone deacetylase-independent transcriptional repression by methyl-CpG-binding protein 2. Nucleic acids research 28, 2201-2206.

Zhu, X., Bergles, D.E., and Nishiyama, A. (2008a). NG2 cells generate both oligodendrocytes and gray matter astrocytes. Development 135, 145-157. 
Zhu, X., Hill, R.A., and Nishiyama, A. (2008b). NG2 cells generate oligodendrocytes and gray matter astrocytes in the spinal cord. Neuron glia biology 4, 19-26. 